\title{
Cationic DABCO-based Catalyst for Site-Selective C-H Alkylation via Photoinduced Hydrogen-Atom Transfer
}

\author{
Akira Matsumoto, ${ }^{* \dagger}{ }^{\dagger}$ Masanori Yamamoto, ${ }^{\dagger}$ and Keiji Maruoka*,†,* \\ ${ }^{\dagger}$ Graduate School of Pharmaceutical Sciences, Kyoto University, Sakyo, Kyoto, \\ 606-8501, Japan \\ ${ }^{t}$ School of Chemical Engineering and Light Industry, Guangdong University of Technology, \\ Guangzhou, 510006, China
}

Email: matsumoto.akira.3c@kyoto-u.ac.jp; maruoka.keiji.4w@kyoto-u.ac.jp 
$\begin{array}{ll}\text { 1. General Information } & \text { S3 }\end{array}$

2. Procedures for Preparation of HAT Catalysts

$\begin{array}{lr}\text { 3. Preparation of Substrates } & \text { S10 }\end{array}$

4. Investigations of Reaction Conditions $\quad$ S11

5. General Procedures for Photoinduced C-H Alkylation via HAT

6. Further Investigations of Substrate Scope

8. Cyclic Voltammetry Measurements 


\section{General Information}

\section{Materials}

Dry solvents were purchased and used as received: tetrahydrofuran (THF), dichloromethane $\left(\mathrm{CH}_{2} \mathrm{Cl}_{2}\right)$, dimethyl formamide (DMF), diethyl ether $\left(\mathrm{Et}_{2} \mathrm{O}\right)$, and acetonitrile $(\mathrm{MeCN})$ were purchased as "Super Dehydrated" grade from FUJIFILM Wako Pure Chemical Co., Inc. 1,2-Dichloroethane (DCE) was purchased as "Dehydrated" grade from Kanto Chemical Co., Inc. Commercially available reagents were purchased from FUJIFILM Wako, Sigma-Aldrich, nacalai tesque, BLDpharm, and TCI, and used as received for the reactions.

\footnotetext{
Method

${ }^{1} \mathrm{H}$ NMR spectra were recorded on a JEOL JNM-AL400 (400 MHz) spectrometer. Data for ${ }^{1} \mathrm{H}$ NMR are reported as follows: chemical shifts in ppm relative to tetramethylsilane as an internal standard in $\mathrm{CDCl}_{3}$, integration, multiplicity ( $\mathrm{s}=$ singlet, $\mathrm{d}=$ doublet, $\mathrm{t}=$ triplet, $\mathrm{q}=$ quartet, $\mathrm{m}=$ multiplet, $\mathrm{br}=$ broad), coupling constants $(\mathrm{Hz})$, and assignment. ${ }^{13} \mathrm{C}$ NMR spectra were recorded on a JEOL JNM-AL400 (100 MHz) spectrometer with complete proton decoupling. Chemical shifts for ${ }^{13} \mathrm{C}$ NMR were reported in ppm relative to the residual solvent as an internal standard. High-resolution mass spectrometry (HRMS) was performed on a Thermo Exactive plus (ESI) spectrometer. For thin layer chromatography (TLC), Merck pre-coated TLC plates (Merck 60 F254) were used, and compounds were visualized with a UV light at $254 \mathrm{~nm}$. Further visualization was achieved by basic aqueous $\mathrm{KMnO}_{4}$ solution stain or phosphomolybdic acid stain. The products were purified by flash column chromatography on neutral silica gel 60 (Kanto Chemical Co. Inc., 40-50 $\mu \mathrm{m}$ ). Further purification by preparative thin layer chromatography (PLC) was performed using Merck silica gel (PTLC 60 F254, $0.5 \mathrm{~mm}$ ) if necessary.
} 


\section{Procedures for Preparation of HAT Catalysts}

Procedure 1

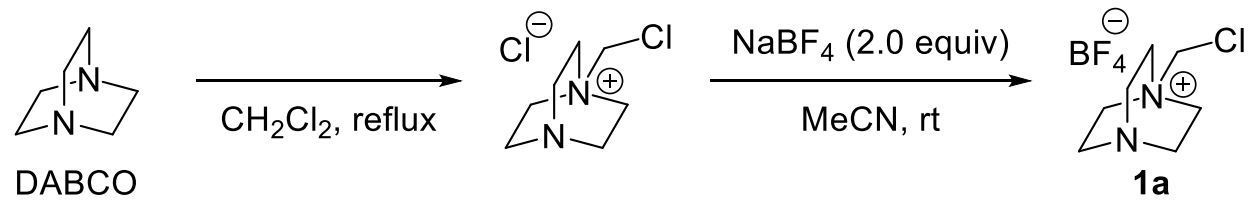

1,4-Diazabicyclo[2.2.2] octane (DABCO, $561 \mathrm{mg}, 5.0 \mathrm{mmol}$ ) was dissolved in $\mathrm{CH}_{2} \mathrm{Cl}_{2}(2.0 \mathrm{~mL})$. The solution was heated at $50^{\circ} \mathrm{C}$ and stirred for 24 hours. The resultant white solid was filtered, washed repeatedly with $\mathrm{CH}_{2} \mathrm{Cl}_{2}$, and dried under vacuum. The obtained product was suspended in $\mathrm{MeCN}$ (5.0 mL), and $\mathrm{NaBF}_{4}$ $(1.10 \mathrm{~g}, 10 \mathrm{mmol})$ was added to the suspension. After being stirred vigorously at room temperature for 20 hours, the mixture was filtered and washed repeatedly with cold $\mathrm{MeCN}$. The filtrate was concentrated under reduced pressure, and the obtained solid was purified by decantation with $\mathrm{Et}_{2} \mathrm{O}$ to afford sufficiently pure catalyst as a white solid.

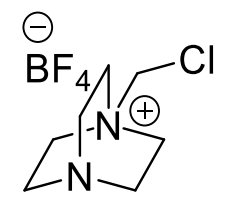

1-(Chloromethyl)-1,4-diazabicyclo[2.2.2] octan-1-ium Tetrafluoroborate (1a) [CAS: 140681-69-2] Procedure 1: colorless solid, $92 \%$ yield (1.14 g).

${ }^{1}$ H NMR (400 MHz, CD $\mathbf{3}$ CN) $\delta 4.94$ (s, 2H), 3.36-3.32 (m, 6H), 3.18-3.32 (m, 6H); ${ }^{13} \mathbf{C ~ N M R ~ ( 1 0 0 ~ M H z , ~}$ $\mathbf{C D}_{3} \mathrm{CN}$ ) $\delta$ 69.3, 52.4, 45.5; HRMS (ESI) Calcd. For $\mathrm{C}_{7} \mathrm{H}_{14} \mathrm{~N}_{2} \mathrm{Cl}$ : 161.0840 ([M - BF $]^{+}$). Found: 161.0841. The spectral data matched those reported in the literature. ${ }^{1}$

\section{Procedure 2}

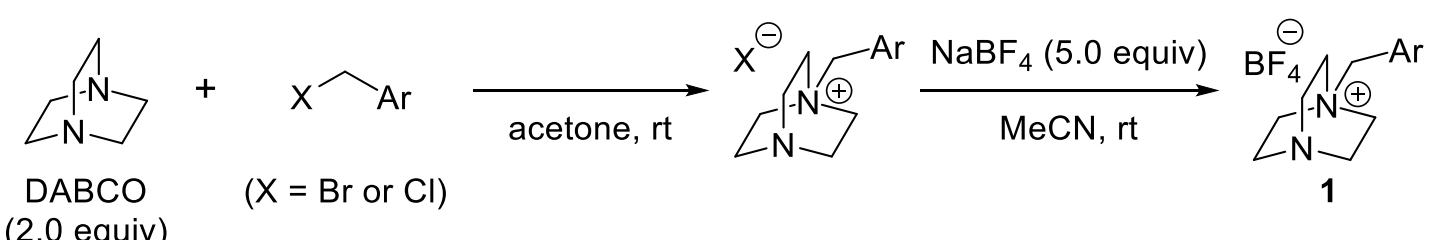

(2.0 equiv)

To a solution of benzyl halide $(5.0 \mathrm{mmol})$ in acetone $(20 \mathrm{~mL})$ was added 1,4-diazabicyclo[2.2.2]octane (DABCO, $1.12 \mathrm{~g}, 10 \mathrm{mmol}$ ) in one portion at room temperature, and the mixture was stirred vigorously for 6 hours under argon atmosphere. The resultant white solid was filtered, washed repeatedly with $\mathrm{Et}_{2} \mathrm{O}$, and dried under vacuum. The obtained product was suspended in $\mathrm{MeCN}(20 \mathrm{~mL})$, and $\mathrm{NaBF}_{4}(2.74 \mathrm{~g}, 25 \mathrm{mmol})$ was added to the suspension. After being stirred vigorously at room temperature for 14 hours, the mixture was filtered and washed repeatedly with cold $\mathrm{MeCN}$. The filtrate was concentrated under reduced pressure, and the obtained solid was purified by decantation with $\mathrm{Et}_{2} \mathrm{O}$ to afford sufficiently pure catalyst as a white solid. 


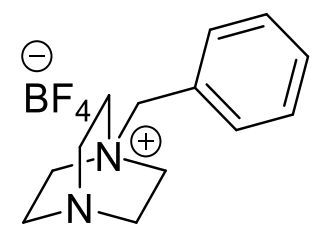

1-Benzyl-1,4-diazabicyclo[2.2.2] octan-1-ium Tetrafluoroborate (1b) [CAS: 42790-21-6] ${ }^{2}$

Procedure 2 with benzyl bromide: colorless solid, 97\% yield (1.40 g).

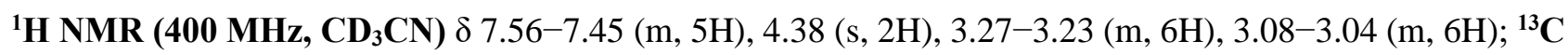
NMR (100 MHz, CD $\left.\mathbf{C D}_{3} \mathrm{CN}\right) \delta 134.1,131.6,130.2,127.5,68.9,53.2,45.8$; HRMS (ESI) Calcd. For $\mathrm{C}_{13} \mathrm{H}_{19} \mathrm{~N}_{2}$ : $203.1543\left(\left[\mathrm{M}-\mathrm{BF}_{4}\right]^{+}\right)$. Found: 203.1551.

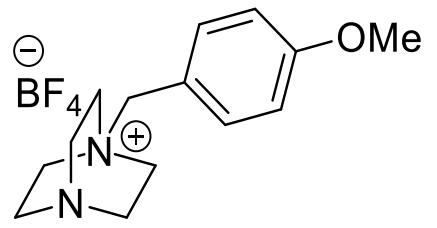

1-(4-Methoxybenzyl)-1,4-diazabicyclo[2.2.2]octan-1-ium Tetrafluoroborate (1c)

Procedure 2 with 4-methoxybenzyl bromide: colorless solid, 95\% yield (1.52 g).

${ }^{1}$ H NMR (400 MHz, CD 3 CN) $\delta$ 7.40-7.36 (m, 2H), 7.05-7.01 (m, 2H), 4.31 (s, 2H), 3.82 (s, 3H), 3.24-3.19 (m, 6H), 3.08-3.02 (m, 6H); ${ }^{13} \mathbf{C}$ NMR (100 MHz, CD $\left.\mathbf{C N N}\right) \delta 162.3,135.6,119.3,115.4,68.6,56.2,52.9$, 45.8; HRMS (ESI) Calcd. For $\mathrm{C}_{14} \mathrm{H}_{21} \mathrm{ON}_{2}: 233.1648\left(\left[\mathrm{M}-\mathrm{BF}_{4}\right]^{+}\right)$. Found: 233.1673.

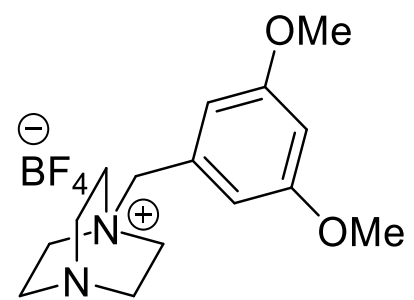

\section{1-(3,5-Dimethoxybenzyl)-1,4-diazabicyclo[2.2.2]octan-1-ium Tetrafluoroborate (1d)}

Procedure 2 with 3,5-dimethoxybenzyl bromide: colorless solid, $85 \%$ yield (1.48 g).

${ }^{1}$ H NMR (400 MHz, CD 3 CN) $\delta$ 6.63-6.60 (m, 3H), 4.23 (s, 2H), 3.80 (s, 6H), 3.29-3.23 (m, 6H), 3.09-3.03 $(\mathrm{m}, 6 \mathrm{H}) ;{ }^{13} \mathbf{C}$ NMR (100 MHz, CD $\left.\mathbf{C N N}\right) \delta 162.2,129.4,111.9,103.0,69.0,56.3,53.4,45.8$; HRMS (ESI) Calcd. For $\mathrm{C}_{15} \mathrm{H}_{24} \mathrm{O}_{2} \mathrm{~N}_{2}: 264.1832\left(\left[\mathrm{M}-\mathrm{BF}_{4}+\mathrm{H}\right]^{2+}\right)$. Found: 264.1831.

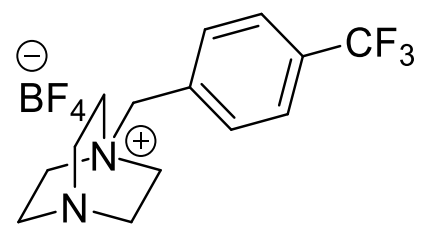

1-(4-(Trifluoromethyl)benzyl)-1,4-diazabicyclo[2.2.2]octan-1-ium Tetrafluoroborate (1e) 
Procedure 2 with 4-trifluoromethylbenzyl bromide: colorless solid, 98\% yield (1.75 g).

${ }^{1} \mathbf{H}$ NMR (400 MHz, CD $\left.\mathbf{C N}\right) \delta$ 7.85-7.81 (m, 2H), 7.69-7.65 (m, 2H), $4.46(\mathrm{~s}, 2 \mathrm{H}), 3.30-3.25(\mathrm{~m}, 6 \mathrm{H})$, 3.11-3.04 (m, 6H); ${ }^{13} \mathbf{C}$ NMR (100 MHz, CD $\left.3 \mathbf{C N}\right) \delta 135.1,132.7$ (q, $\left.J=32.5 \mathrm{~Hz}\right), 132.0,127.0(\mathrm{q}, J=3.9$ $\mathrm{Hz}), 125.1$ (q, $J=269.9 \mathrm{~Hz}), 67.9,53.4,45.8$; HRMS (ESI) Calcd. For $\mathrm{C}_{14} \mathrm{H}_{19} \mathrm{~N}_{2} \mathrm{~F}_{3}: 271.1417\left(\left[\mathrm{M}-\mathrm{BF}_{4}\right]^{+}\right)$. Found: 271.1462.<smiles></smiles>

1-(3,5-Bis(trifluoromethyl)benzyl)-1,4-diazabicyclo[2.2.2]octan-1-ium Tetrafluoroborate (1f) [CAS:

\section{3-01-1]}

Procedure 2 with 3,5-bis(trifluoromethyl)benzyl chloride: colorless solid, 55\% yield (1.17 g).

${ }^{1}$ H NMR (400 MHz, CD 3 CN) $\delta 8.19$ (s, 1H), 8.07 (s, 2H), 4.53 (s, 2H), 3.31-3.26 (m, 6H), 3.12-3.06 (m, 6H); ${ }^{13}$ C NMR (100 MHz, CD $\mathbf{C N}$ ) $\delta 134.8,132.9$ (q, $\left.J=34.3 \mathrm{~Hz}\right), 130.5,125.6$ (q, $\left.J=3.9 \mathrm{~Hz}\right), 124.2$ (q, $J$ $=270.8 \mathrm{~Hz}), 67.0,53.4,45.8$; HRMS (ESI) Calcd. For $\mathrm{C}_{15} \mathrm{H}_{19} \mathrm{~N}_{2} \mathrm{~F}_{6} 341.1447\left(\left[\mathrm{M}-\mathrm{BF}_{4}+2 \mathrm{H}\right]^{3+}\right)$. Found: 341.1448. The spectral data matched those reported in the literature. ${ }^{3}$

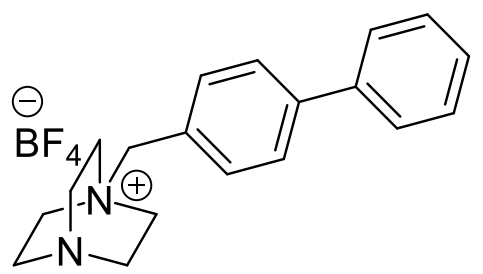

\section{1-([1,1'-Biphenyl]-4-ylmethyl)-1,4-diazabicyclo[2.2.2]octan-1-ium Tetrafluoroborate (1g)}

Procedure 2 with 4-phenylbenzyl bromide: colorless solid, 98\% yield (1.79 g).

${ }^{1}$ H NMR (400 MHz, CD 3 CN) $\delta$ 7.80-7.76 (m, 2H), 7.71-7.67 (m, 2H), 7.56-7.47 (m, 4H), 7.45-7.39 (m, 1H), 4.41 (s, 2H), 3.31-3.35 (m, 6H), 3.12-3.05 (m, 6H); ${ }^{13} \mathbf{C}$ NMR (100 MHz, CD $\left.\mathbf{C N}\right) \delta 143.9,140.4,134.7$, $130.1,129.2,128.5,128.0,126.6,68.6,53.3,45.8$; HRMS (ESI) Calcd. For $\mathrm{C}_{19} \mathrm{H}_{24} \mathrm{~N}_{2}: 280.1934\left(\left[\mathrm{M}-\mathrm{BF}_{4}+\right.\right.$ $\mathrm{H}]^{2+}$ ). Found: 280.1943.

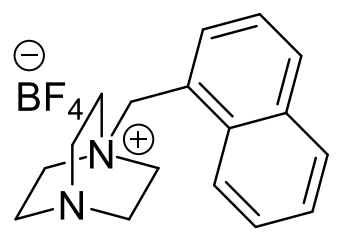


Procedure 2 with 1-(chloromethyl)naphthalene: colorless solid, 99\% yield (1.70 g).

${ }^{1}$ H NMR (400 MHz, CD ${ }_{3}$ CN) $\delta 8.22(\mathrm{~d}, J=8.0 \mathrm{~Hz}, 1 \mathrm{H}), 8.11(\mathrm{~d}, J=8.0 \mathrm{~Hz}, 1 \mathrm{H}), 8.03(\mathrm{~d}, J=8.0 \mathrm{~Hz}, 1 \mathrm{H})$, 7.73-7.67 (m, 2H), 7.65-7.59 (m, 2H), $4.86(\mathrm{~s}, 2 \mathrm{H}), 3.35-3.28(\mathrm{~m}, 6 \mathrm{H}), 3.08-2.99(\mathrm{~m}, 6 \mathrm{H}) ;{ }^{13} \mathbf{C}$ NMR (100 MHz, CD ${ }_{3}$ CN) $\delta 135.1,135.0,134.0,132.8,130.2,128.6,127.5,126.3,124.5,123.6,65.4,53.6,46.0$; HRMS (ESI) Calcd. For $\mathrm{C}_{17} \mathrm{H}_{21} \mathrm{~N}_{2}: 253.1699\left(\left[\mathrm{M}-\mathrm{BF}_{4}\right]^{+}\right)$. Found: 253.1731.

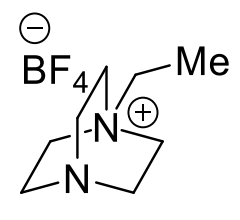

\section{1-Ethyl-1,4-diazabicyclo[2.2.2]octan-1-ium Tetrafluoroborate (1j)}

Procedure 2 with bromoethane: colorless solid (hygroscopic), $75 \%$ yield $(852 \mathrm{mg}$ ).

${ }^{1} \mathrm{H}$ NMR (400 MHz, CD 3 CN) $\delta 3.21(\mathrm{q}, J=6.8 \mathrm{~Hz}, 2 \mathrm{H}), 3.20-3.14(\mathrm{~m}, 6 \mathrm{H}), 3.12-3.06(\mathrm{~m}, 6 \mathrm{H}), 1.26(\mathrm{tt}, J=$ 6.8, $2.4 \mathrm{~Hz}, 3 \mathrm{H}) ;{ }^{13} \mathbf{C}$ NMR (100 MHz, $\left.\mathbf{C D}_{3} \mathbf{C N}\right) \delta 60.8,52.8,45.8$; HRMS (ESI) Calcd. For $\mathrm{C}_{8} \mathrm{H}_{17} \mathrm{~N}_{2}$ : $141.1386\left(\left[\mathrm{M}-\mathrm{BF}_{4}\right]^{+}\right)$. Found: 141.1388 .

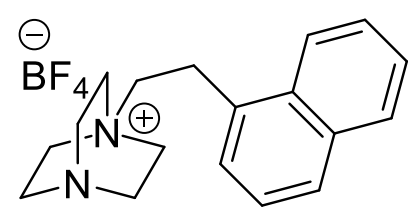

\section{1-(2-(Naphthalen-1-yl)ethyl)-1,4-diazabicyclo[2.2.2] octan-1-ium tetrafluoroborate (1k)}

Procedure 2 with 1-(2-bromoethyl)naphthalene: colorless solid, 94\% yield (1.58 g).

${ }^{1}$ H NMR (400 MHz, CD ${ }_{3}$ CN) $\delta 8.04(\mathrm{~d}, J=8.8 \mathrm{~Hz}, 1 \mathrm{H}), 7.95(\mathrm{~d}, J=7.6 \mathrm{~Hz}, 1 \mathrm{H}), 7.89-7.84(\mathrm{~m}, 1 \mathrm{H})$, 7.65-7.60 (m, 1H), 7.59-7.55 (m, 1H), 7.50-7.47 (m, 2H), 3.56-3.49 (m, 2H), 3.49-3.43 (m, 2H), 3.43-3.37 (m, 6H), 3.21-3.14 (m, 6H); ${ }^{13} \mathbf{C}$ NMR (100 MHz, CD $\mathbf{C N}$ ) $\delta 135.0,132.8,132.4,130.0,129.1,128.4,127.7$, 127.1, 126.8, 124.1, 65.0, 53.4, 45.8, 25.7; HRMS (ESI) Calcd. For $\mathrm{C}_{18} \mathrm{H}_{24} \mathrm{~N}_{2}: 268.1934\left(\left[\mathrm{M}-\mathrm{BF}_{4}+\mathrm{H}\right]^{2+}\right)$. Found: 268.1934. 


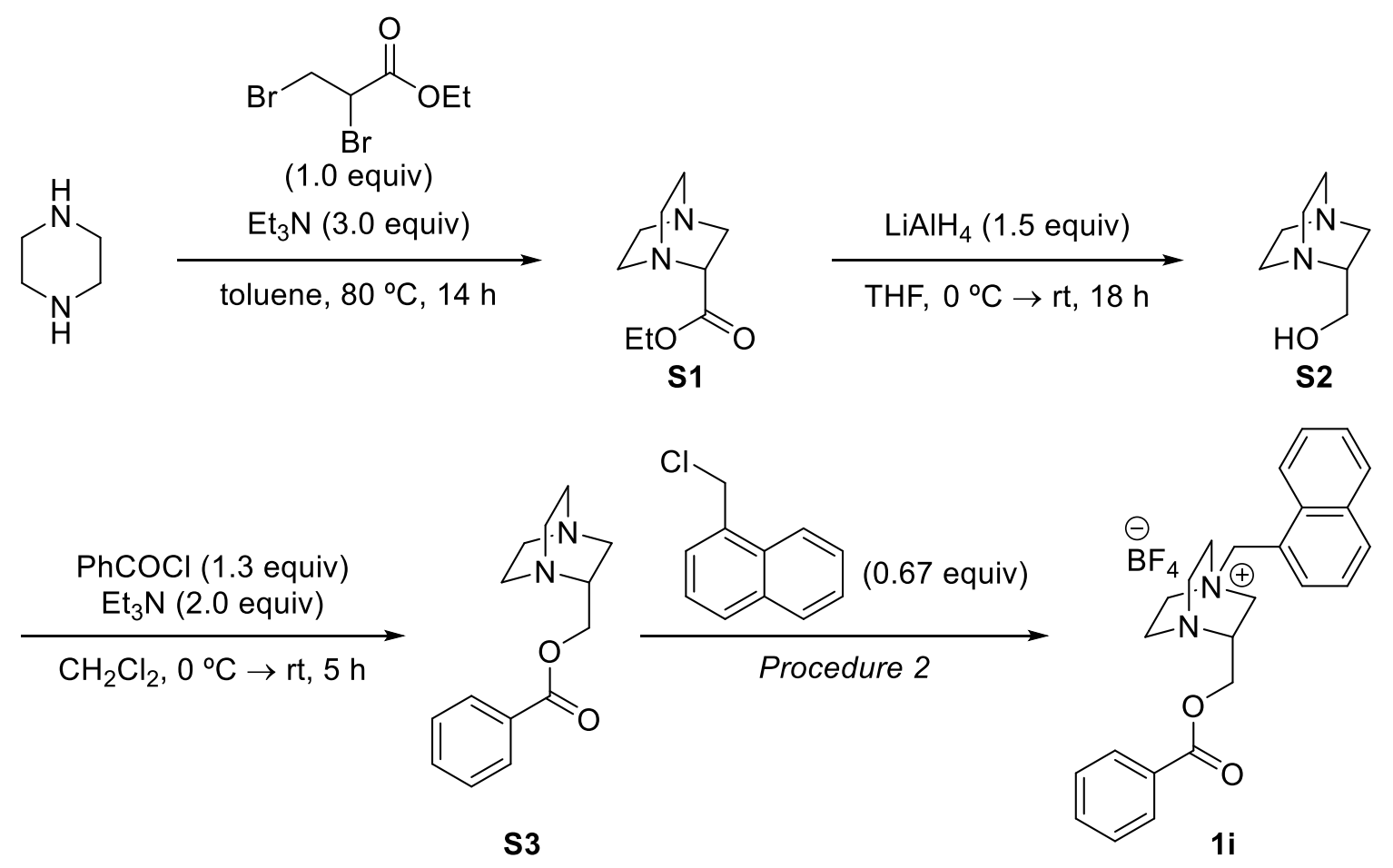

To a solution of piperazine $(2.58 \mathrm{~g}, 30 \mathrm{mmol})$ in toluene $(100 \mathrm{~mL})$ were added triethylamine $(12.5 \mathrm{~mL}, 30$ mmol) and ethyl 2,3-dibromopropionate $(4.35 \mathrm{~mL}, 30 \mathrm{mmol})$ under argon atmosphere at room temperature. The mixture was heated at $80^{\circ} \mathrm{C}$ and stirred for 14 hours under argon atmosphere. The mixture was cooled to room temperature and filtered. The filter cake was repeatedly washed with EtOAc, and the filtrate was concentrated under reduced pressure. $\mathrm{H}_{2} \mathrm{O}(30 \mathrm{~mL})$ was added to the crude residue, and the mixture was extracted with EtOAc $(3 \times 30 \mathrm{~mL})$. The combined organic layers were washed with brine, dried over anhydrous $\mathrm{Na}_{2} \mathrm{SO}_{4}$, and concentrated under reduced pressure. The obtained crude product was purified by flash silica-gel column chromatography using EtOAc and then $\mathrm{CHCl}_{3} / \mathrm{MeOH}(\mathrm{v} / \mathrm{v}=10: 1)$ as eluents to afford ethyl 1,4diazabicyclo[2.2.2] octane-2-carboxylate (S1, $3.13 \mathrm{~g}, 57 \%$ yield) as a brown oil. The spectral data matched those reported in the literature. ${ }^{4}$

To a suspension of lithium aluminum hydride $(740 \mathrm{mg}, 19.5 \mathrm{mmol})$ in anhydrous THF $(10 \mathrm{~mL})$ was added a solution of ethyl 1,4-diazabicyclo[2.2.2] octane-2-carboxylate (S1, 2.40 g, $13.0 \mathrm{mmol})$ in anhydrous THF (10 $\mathrm{mL}$ ) dropwise at $0{ }^{\circ} \mathrm{C}$ under argon atmosphere. The mixture was warmed to room temperature and stirred for $18 \mathrm{~h}$. To the reaction solution were added slowly $\mathrm{H}_{2} \mathrm{O}(800 \mu \mathrm{L}), 15 \% \mathrm{NaOH}$ aq. $(800 \mu \mathrm{L})$, and then a further $\mathrm{H}_{2} \mathrm{O}(800 \mu \mathrm{L})$. The mixture was stirred for 1 hour, and the resultant solid was filtered off. The filter cake was repeatedly washed with $\mathrm{CHCl}_{3}$, and the filtrate was concentrated under reduced pressure to afford (1,4diazabicyclo[2.2.2] octan-2-yl)methanol (S2, $1.70 \mathrm{~g}, 99 \%$ yield) as a colorless oil. The obtained product was used for the next step without further purification. The spectral data matched those reported in the literature. ${ }^{4}$

To a solution of (1,4-diazabicyclo[2.2.2] octan-2-yl)methanol (S2, $569 \mathrm{mg}, 4.0 \mathrm{mmol})$ and triethylamine (1.1 $\mathrm{mL}, 8.0 \mathrm{mmol})$ in $\mathrm{CH}_{2} \mathrm{Cl}_{2}(20 \mathrm{~mL})$ was added benzoyl chloride $(599 \mu \mathrm{L}, 5.2 \mathrm{mmol})$ dropwise at $0{ }^{\circ} \mathrm{C}$ under argon atmosphere. The mixture was warmed to room temperature and stirred for $5 \mathrm{~h}$. To the reaction solution 
was added saturated $\mathrm{NaHCO}_{3}$ aq. $(20 \mathrm{~mL})$, and the mixture was extracted with EtOAc $(3 \times 20 \mathrm{~mL})$. The combined organic layers were washed with brine, dried over anhydrous $\mathrm{Na}_{2} \mathrm{SO}_{4}$, and concentrated under reduced pressure. The obtained crude product was purified by flash silica-gel column chromatography using EtOAc and then $\mathrm{CHCl}_{3} / \mathrm{MeOH}(\mathrm{v} / \mathrm{v}=10: 1$ to 3/1) as eluents to afford (1,4-diazabicyclo[2.2.2] octan-2yl)methyl benzoate (S3, $861 \mathrm{mg}, 87 \%$ yield) as a colorless oil: [CAS: 1427392-91-3] ${ }^{1} \mathbf{H}$ NMR (400 MHz, $\left.\mathbf{C D C l}_{3}\right) \delta 8.07-8.01(\mathrm{~m}, 2 \mathrm{H}), 7,58-7.52(\mathrm{~m}, 1 \mathrm{H}), 7.46-7.40(\mathrm{~m}, 2 \mathrm{H}), 4.50(\mathrm{dd}, J=11.2,7.6 \mathrm{~Hz}, 1 \mathrm{H}), 4.32(\mathrm{dd}$, $J=11.2,6.4 \mathrm{~Hz}, 1 \mathrm{H}), 3.25-3.17(\mathrm{~m}, 1 \mathrm{H}), 3.10-3.01(\mathrm{~m}, 2 \mathrm{H}), 2.93-2.88(\mathrm{~m}, 2 \mathrm{H}), 2.83-2.65(\mathrm{~m}, 5 \mathrm{H})$, $2.55-2.25(\mathrm{~m}, 2 \mathrm{H})$.

The synthesis of $\mathbf{1 i}$ from $\mathbf{S 3}$ was followed by procedure 2 using 1-(chloromethyl)naphthalene as a benzyl halide.

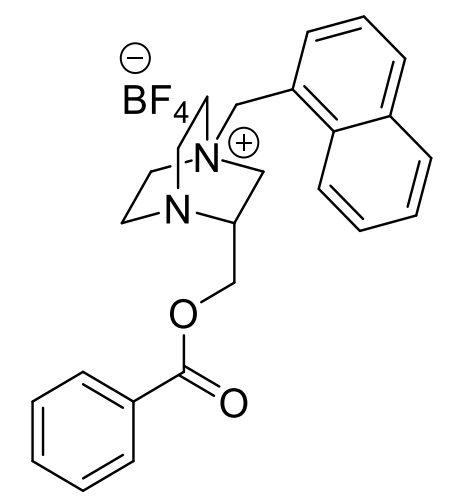

\section{3-((Benzoyloxy)methyl)-1-(naphthalen-1-ylmethyl)-1,4-diazabicyclo[2.2.2]octan-1-ium Tetrafluoroborate (1i)}

Procedure 2 with $1.3 \mathrm{mmol}$ of 1-(chloromethyl)naphthalene: colorless solid, 99\% yield from $\mathbf{S 3}(617 \mathrm{mg})$.

${ }^{1}$ H NMR (400 MHz, CD $\left.{ }_{3} \mathrm{CN}\right) \delta 8.23(\mathrm{~d}, J=8.8 \mathrm{~Hz}, 1 \mathrm{H}), 8.11(\mathrm{~d}, J=8.8 \mathrm{~Hz}, 1 \mathrm{H}), 8.03(\mathrm{~d}, J=8.8 \mathrm{~Hz}, 1 \mathrm{H})$, 7.92-7.88 (m, 2H), 7.75-7.60 (m, 5H), 7.52-7.47 (m, 2H), $4.93(\mathrm{~d}, J=14.0 \mathrm{~Hz}, 1 \mathrm{H}), 4.88(\mathrm{~d}, J=13.2 \mathrm{~Hz}$, $1 \mathrm{H}), 4.47$ (dd, $J=11.6,7.2 \mathrm{~Hz}, 1 \mathrm{H}), 4.20$ (dd, $J=11.6,6.0 \mathrm{~Hz}, 1 \mathrm{H}), 3.63-3.50(\mathrm{~m}, 2 \mathrm{H}), 3.44-3.23(\mathrm{~m}, 5 \mathrm{H})$, 3.16-3.08 (m, 3H), 3.03-2.95 (m, 1H); ${ }^{13} \mathbf{C}$ NMR (100 MHz, CD 3 CN) $\delta 166.8,135.2,135.0,134.4,134.0$, $132.9,130.7,130.33,130.25,129.7,128.7,127.5,126.3,124.5,123.5,65.5,64.0,55.7,53.9,53.8,53.1,47.8$, 41.1; HRMS (ESI) Calcd. For $\mathrm{C}_{25} \mathrm{H}_{29} \mathrm{O}_{2} \mathrm{~N}_{2}: 389.2224\left(\left[\mathrm{M}-\mathrm{BF}_{4}+2 \mathrm{H}\right]^{3+}\right)$. Found: 389.2257. 


\section{Preparation of Substrates}<smiles>COC(C)c1ccccc1</smiles>

\section{(1-Methoxyethyl)benzene [CAS: 4013-34-7]}

The title compound was prepared according to the literature. ${ }^{5}$

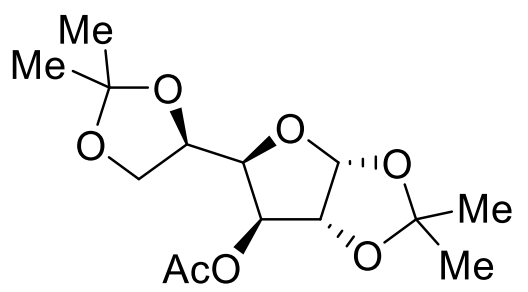

3-O-Acetyl-1,2:5,6-di- $O$-isopropylidene- $\alpha$-D-glucofuranose [CAS: 16713-80-7]

The title compound was prepared according to the literature. ${ }^{6}$<smiles>CC(C)CCCC(C)CCOC(=O)c1ccccc1</smiles>

3,7-Dimethyloctyl benzoate [CAS: 9444-18-5]

The title compound was prepared according to the literature. ${ }^{7}$ 


\section{Investigations of Reaction Conditions}

\section{4-1. Screening of Photoredox Catalysts}

Table S1. Screening of photoredox catalysts.

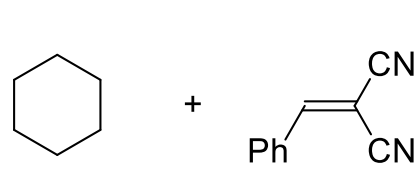

(3.0 equiv)

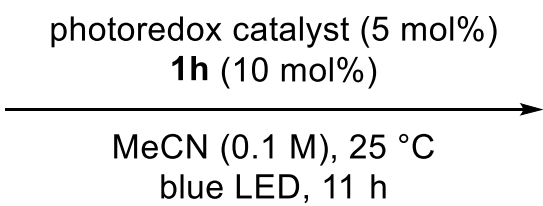

blue LED, $11 \mathrm{~h}$

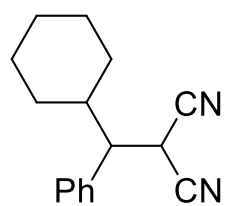

3<smiles></smiles>

Mes-Acr ${ }^{+}$<smiles></smiles>

Acr-1<smiles></smiles>

Acr-2

${ }^{*} E_{\text {red }}\left({ }^{*} \mathrm{Acr}^{+} / \mathrm{Acr}^{\Sigma}\right)=+2.15 \mathrm{~V}$

$E_{\text {red }}\left(\mathrm{Acr}^{+} / \mathrm{Acr}^{\Sigma}\right)=-0.59 \mathrm{~V}$

$$
\begin{gathered}
{ }^{*} E_{\text {red }}\left({ }^{*} \mathrm{Acr}^{+} / \mathrm{Acr}^{\Sigma}\right)=+2.09 \mathrm{~V} \\
E_{\text {red }}\left(\mathrm{Acr}^{+} / \mathrm{Acr}^{\Sigma}\right)=-0.57 \mathrm{~V}
\end{gathered}
$$<smiles></smiles>

${ }^{*} E_{\text {red }}\left({ }^{*} \mathrm{Acr}^{+} / \mathrm{Acr}^{\Sigma}\right)=+1.62 \mathrm{~V}$

$E_{\text {red }}\left(\mathrm{Acr}^{+} / \mathrm{Acr}^{\Sigma}\right)=-0.84 \mathrm{~V}$

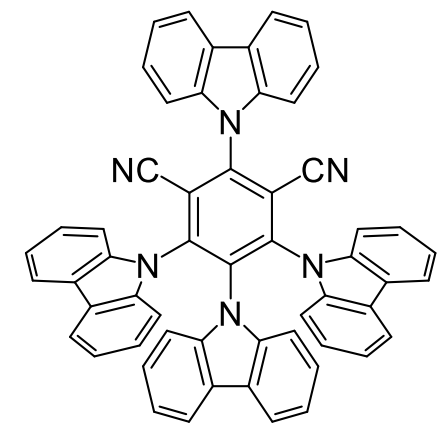

4CzIPN

${ }^{*} E_{\text {red }}\left({ }^{*} \mathrm{Acr}^{+} / \mathrm{Acr}^{\Sigma}\right)=+2.09 \mathrm{~V}$

$E_{\text {red }}\left(\mathrm{Acr}^{+} / \mathrm{Acr}^{\Sigma}\right)=-0.58 \mathrm{~V}$

\begin{tabular}{cccc}
\hline entry & photoredox catalyst & conv. of 2 $(\%)$ & yield of 3 $(\%)$ \\
\hline 1 & Mes-Acr+ & 99 & 95 \\
2 & Acr-1 & 99 & 88 \\
3 & Acr-2 & 99 & 87 \\
4 & Acr-3 & $<5$ & trace \\
5 & 4CzIPN & $<5$ & trace \\
\hline
\end{tabular}




\section{4-2. Comparison of tert-Amine-derived HAT Catalysts}

Table S2. Screening of tert-amine-derived HAT catalysts.

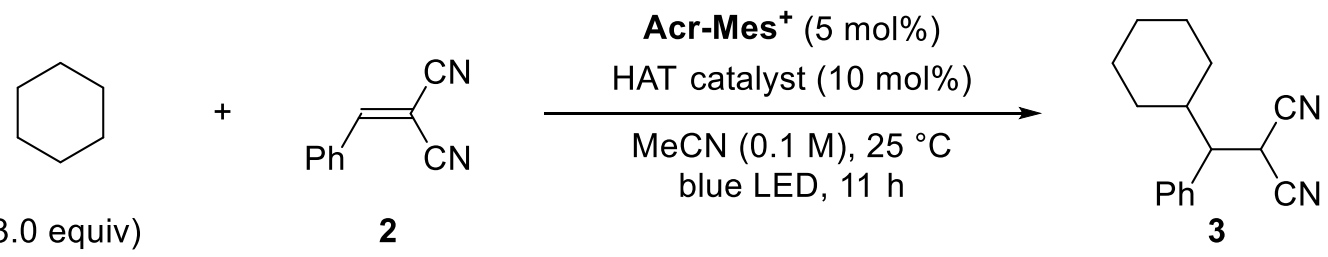

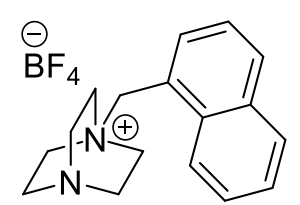

$1 \mathrm{~h}$

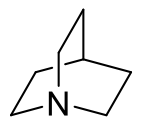

HAT-1

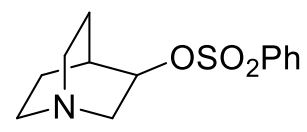

HAT-2<smiles>C1CN2CCN1CC2</smiles>

HAT-3

\begin{tabular}{cccc}
\hline entry & HAT catalyst & conv. of 2 (\%) & yield of $\mathbf{3}(\%)$ \\
\hline 1 & 1h & 99 & 95 \\
2 & HAT-1 & 16 & 6 \\
3 & HAT-2 & 37 & 3 \\
4 & HAT-3 & 20 & N.D. \\
\hline
\end{tabular}




\section{General Procedures for Photoinduced C-H Alkylation via HAT}

\section{General Procedure 1}

To an oven-dried glass tube equipped with a magnetic stir bar were added 9-mesityl-3,6-di-tert-butyl-10phenylacridinium tetrafluoroborate (Mes-Acr $\left.{ }^{+}, 5.7 \mathrm{mg}, 0.010 \mathrm{mmol}, 5.0 \mathrm{~mol} \%\right)$, 1-(naphthalen-1-ylmethyl)1,4-diazabicyclo[2.2.2] octan-1-ium tetrafluoroborate $(\mathbf{1 h}, 6.8 \mathrm{mg}, 0.020 \mathrm{mmol}, 10 \mathrm{~mol} \%), \mathrm{C}-\mathrm{H}$ substrate (3.0-20 equiv), and a radical acceptor $(0.20 \mathrm{mmol})$. The tube was capped with a rubber septum equipped with argon balloon and LED light source (Techno-Sigma, PER-AMP, $448 \mathrm{~nm}$ ), and degassed via vacuum evacuation and subsequent backfill with argon for three times. Dry MeCN $(2.0 \mathrm{~mL})$ was then added via syringe and the cap was sealed with parafilm. The tube was covered with aluminum foil, set in a temperature-controlled reactor (Techno-Sigma, UCR-80 Nh), and stirred under the irradiation of blue LED while keeping the temperature at $25^{\circ} \mathrm{C}$. After being stirred for $11 \mathrm{~h}$, the reaction mixture was diluted with $\mathrm{Et}_{2} \mathrm{O}$ and passed through a short silicagel pad eluting with $\mathrm{Et}_{2} \mathrm{O}$. The solution was evaporated and the obtained crude mixture was purified by flash column chromatography on silica-gel using hexane/EtOAc as an eluent to afford the desired product.

\section{General Procedure 2}

To an oven-dried glass tube equipped with a magnetic stir bar were added 9-mesityl-3,6-di-tert-butyl-10phenylacridinium tetrafluoroborate $\left(\mathbf{M e s}_{-} \mathbf{A c r}^{+}, 5.7 \mathrm{mg}, 0.010 \mathrm{mmol}, 5 \mathrm{~mol} \%\right)$, HAT catalyst $(0.020 \mathrm{mmol}, 10$ mol\%), and $\mathrm{C}-\mathrm{H}$ substrate (3.0-20 equiv). The tube was capped with a rubber septum equipped with argon balloon and LED light source (Techno-Sigma, PER-AMP, $448 \mathrm{~nm}$ ), and degassed via vacuum evacuation and subsequent backfill with argon for three times. To the mixture a solution of 1,1,1,3,3,3-hexafluoroisopropyl acrylate $(33.4 \mu \mathrm{L}, 0.20 \mathrm{mmol})$ in dry $\mathrm{MeCN}(2.0 \mathrm{~mL})$ was added via syringe, and the cap was sealed with parafilm. The tube was covered with aluminum foil, set in a temperature-controlled reactor (Techno-Sigma, UCR-80 Nh), and stirred under the irradiation of blue LED while keeping the temperature at $25^{\circ} \mathrm{C}$. After being stirred for $11 \mathrm{~h}$, benzyl alcohol $(41.1 \mu \mathrm{L}, 0.40 \mathrm{mmol}, 2.0$ equiv) or benzylamine (43.7 $\mu \mathrm{L}, 0.40 \mathrm{mmol}, 2.0$ equiv) and 1,8-diazabicyclo[5.4.0]undec-7-ene (DBU, $29.8 \mu \mathrm{L}, 1.0$ equiv) were added to the reaction solution via syringe, and the mixture was further stirred at $40{ }^{\circ} \mathrm{C}$ for $3 \mathrm{~h}$. The mixture was diluted with EtOAc and passed through a short silica-gel pad eluting with EtOAc. The solution was evaporated, and the obtained crude mixture was purified by flash column chromatography on silica-gel using hexane/EtOAc as an eluent to afford the desired product.

\section{General Procedure 3}

To an oven-dried glass tube equipped with a magnetic stir bar were added 9-mesityl-3,6-di-tert-butyl-10phenylacridinium tetrafluoroborate $\left(\mathbf{M e s}_{-} \mathbf{A c r}^{+}, 5.7 \mathrm{mg}, 0.010 \mathrm{mmol}, 5 \mathrm{~mol} \%\right)$, HAT catalyst $(0.020 \mathrm{mmol}, 10$ $\mathrm{mol} \%$ ), and $\mathrm{C}-\mathrm{H}$ substrate (3.0-20 equiv). The tube was capped with a rubber septum equipped with argon balloon and LED light source (Techno-Sigma, PER-AMP, $448 \mathrm{~nm}$ ), and degassed via vacuum evacuation and subsequent backfill with argon for three times. To the mixture a solution of 1,1,1,3,3,3-Heaxafluoroisopropyl acrylate $(33.4 \mu \mathrm{L}, 0.20 \mathrm{mmol})$ in dry $\mathrm{MeCN}(2.0 \mathrm{~mL})$ was added via syringe, and the cap was sealed with 
parafilm. The tube was covered with aluminum foil, set in a temperature-controlled reactor (Techno-Sigma, UCR- $80 \mathrm{Nh}$ ), and stirred under the irradiation of blue LED while keeping the temperature at $25^{\circ} \mathrm{C}$. After being stirred for $11 \mathrm{~h}, \mathrm{MeOH}(2.0 \mathrm{~mL})$ and triethylamine $(55.4 \mu \mathrm{L}, 2.0$ equiv) were added to the reaction solution via syringe, and the mixture was further stirred at $40{ }^{\circ} \mathrm{C}$ for $3 \mathrm{~h}$. The mixture was diluted with EtOAc and passed through a short silica-gel pad eluting with EtOAc. The solution was evaporated, and the obtained crude mixture was purified by flash column chromatography on silica-gel using hexane/EtOAc as an eluent to afford the desired product.
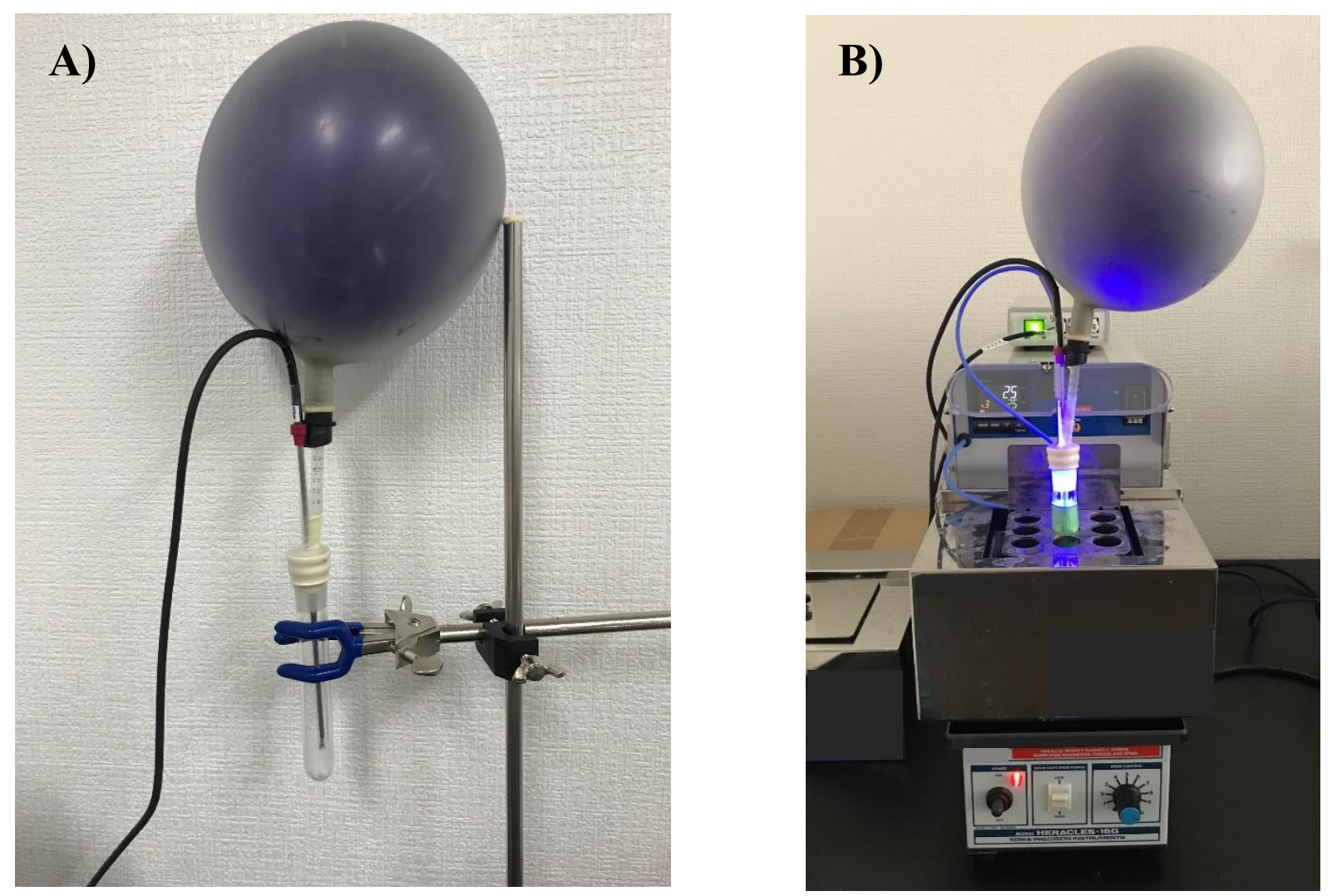

Figure S1. Reaction Setup. A) A LED light source (Techno-Sigma, PER-AMP, $448 \mathrm{~nm}$ ) was placed inside the glass tube, which was capped with a rubber septum and covered with palafilm. B) The tube was covered with aluminum foil and the reaction solution was stirred in a temperature-controlled reactor (Techno-Sigma, UCR-80 Nh) to keep the temperature at $25^{\circ} \mathrm{C}$ under argon atmosphere. 
<smiles>N#CC(C#N)C(c1ccccc1)C1CCCCC1</smiles>

\section{2-(Cyclohexyl(phenyl)methyl)malononitrile (3) [CAS: 545385-91-9]}

General Procedure 1 with cyclohexane $(64.7 \mu \mathrm{L}, 0.60 \mathrm{mmol}, 3.0$ equiv) and benzalmalononitrile (30.8 $\mathrm{mg}$, $0.20 \mathrm{mmol}$ ): colorless oil, $98 \%$ yield $(46.7 \mathrm{mg}) .{ }^{1} \mathbf{H}$ NMR (400 $\left.\mathbf{~ M H z}, \mathbf{C D C l}_{3}\right) \delta$ 7.43-7.34 (m, 3H), 7.33-7.29 $(\mathrm{m}, 2 \mathrm{H}), 4.19(\mathrm{~d}, J=5.6 \mathrm{~Hz}, 1 \mathrm{H}), 2.88(\mathrm{dd}, J=9.6,5.6 \mathrm{~Hz}, 1 \mathrm{H}), 2.07-1.96(\mathrm{~m}, 1 \mathrm{H}), 1.95-1.88(\mathrm{~m}, 1 \mathrm{H})$, $1.88-1.79(\mathrm{~m}, 1 \mathrm{H}), 1.71-1.62(\mathrm{~m}, 2 \mathrm{H}), 1.50-1.42(\mathrm{~m}, 1 \mathrm{H}), 1.36(\mathrm{dt}, J=12.8,3.6 \mathrm{~Hz}, 1 \mathrm{H}), 1.26-0.99(\mathrm{~m}, 3 \mathrm{H})$, 0.88-0.77 (m, 1H); ${ }^{13} \mathbf{C}$ NMR (100 MHz, $\left.\mathbf{C D C l}_{3}\right) \delta$ 136.8, 129.2, 128.8, 128.4, 112.4, 112.1, 52.3, 39.3, 31.2, $30.6,27.2,25.9(2 \mathrm{C}), 25.8$. The spectral data matched those reported in the literature. ${ }^{8}$

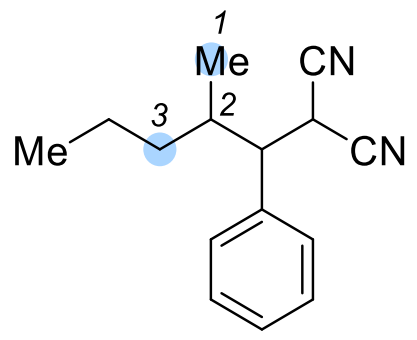

\section{2-(2-Methyl-1-phenylpentyl)malononitrile (4) (containing regio- and diastereoisomers)}

General Procedure 1 with pentane $(68.7 \mu \mathrm{L}, 0.60 \mathrm{mmol}, 3.0$ equiv) and benzalmalononitrile (30.8 $\mathrm{mg}, 0.20$ mmol): colorless oil, $90 \%$ yield $(40.7 \mathrm{mg}, \mathrm{C} 1: \mathrm{C} 2: \mathrm{C} 3=1: 12: 6.7$, d.r. $(\mathrm{C} 2)=2.0: 1)$. Regioisomers could not be separated. ${ }^{1} \mathbf{H}$ NMR (400 MHz, $\left.\mathbf{C D C l}_{3}\right) \delta(\mathrm{C} 2$ product) 7.43-7.27 (m, 5H), 4.19 (d, J = 7.2 Hz, 0.67H), 4.15 $(\mathrm{d}, J=5.2 \mathrm{~Hz}, 0.33 \mathrm{H}), 3.08(\mathrm{dd}, J=10.0,5.2 \mathrm{~Hz}, 0.33 \mathrm{H}), 2.90(\mathrm{dd}, J=10.0,5.2 \mathrm{~Hz}, 0.67 \mathrm{H}), 2.27-2.03(\mathrm{~m}$, 1H), 1.62-1.16 (m, 4H), 1.10 (d, $J=6.4 \mathrm{~Hz}, 2 \mathrm{H}), 0.97$ (d, $J=7.2 \mathrm{~Hz}, 2 \mathrm{H}), 0.78$ (d, $J=7.6 \mathrm{~Hz}, 1 \mathrm{H}), 0.77(\mathrm{~d}, J$ $=7.2 \mathrm{~Hz}, 1 \mathrm{H}) ; \delta(\mathrm{C} 3$ product $) 7.43-7.27(\mathrm{~m}, 5 \mathrm{H}), 4.17(\mathrm{~d}, J=4.4 \mathrm{~Hz}, 1 \mathrm{H}), 3.00(\mathrm{dd}, J=8.8,7.6 \mathrm{~Hz}, 1 \mathrm{H})$, 2.27-2.20 (m, 1H), 1.62-1.16 (m, 4H), $0.79(\mathrm{t}, J=7.2 \mathrm{~Hz}, 6 \mathrm{H}) ;{ }^{13} \mathbf{C} \mathbf{~ N M R}\left(\mathbf{1 0 0} \mathbf{~ M H z}, \mathbf{C D C l}_{3}\right) \delta(\mathrm{C} 2$ product + C3 product) 136.81, 136.75, 136.4, 129.2, 129.1, 128.9, 128.8, 128.52, 128.48, 128.43, 112.4, 112.1, 112.0, 52.4, 51.7, 48.8, 41.2, 36.6, 35.7, 34.61, 34.58, 27.9, 27.8, 27.5, 21.63, 21.56, 19.68, 19.59, 17.5, 16.3, 14.2, 14.1, 10.0, 9.8 ( 2 aromatic peaks are overlapped). The spectral data matched those reported in the literature. ${ }^{9}$<smiles>N#CC(C#N)C(c1ccccc1)C1CCCCCCC1</smiles> 
General Procedure 1 with cyclooctane $(80.2 \mu \mathrm{L}, 0.60 \mathrm{mmol}, 3.0$ equiv) and benzalmalononitrile (30.8 $\mathrm{mg}$, 0.20 mmol): colorless oil, 98\% yield (52.4 mg). ${ }^{1} \mathbf{H}$ NMR (400 MHz, $\left.\mathbf{C D C l}_{3}\right) \delta 7.44-7.30$ (m, 5H), 4.20 (d, $J$ $=5.2 \mathrm{~Hz}, 1 \mathrm{H}), 2.95(\mathrm{dd}, J=10.4,5.2 \mathrm{~Hz}, 1 \mathrm{H}), 2.41-2,27(\mathrm{~m}, 1 \mathrm{H}), 1.87-1.35(\mathrm{~m}, 12 \mathrm{H}), 1.31-1.14(\mathrm{~m}, 2 \mathrm{H})$; ${ }^{13} \mathrm{C}$ NMR (100 MHz, $\left.\mathbf{C D C l}_{3}\right) \delta 137.0,129.2,128.8,128.6,112.4,112.0,52.1,38.2,30.6,28.7,27.9,27.5$, 26.7, 26.4, 25.8, 24.4. The spectral data matched those reported in the literature. ${ }^{9}$<smiles>N#CC(C#N)C(c1ccccc1)C1CCCCCCCCCCC1</smiles>

\section{2-(Cyclododecyl(phenyl)methyl)malononitrile (6) [CAS: 2244443-52-3]}

General Procedure 1 with cyclooctane (101 mg, $0.60 \mathrm{mmol}, 3.0$ equiv) and benzalmalononitrile (30.8 $\mathrm{mg}$, $0.20 \mathrm{mmol}$ ): colorless foam, 74\% yield (48.0 mg). ${ }^{1} \mathbf{H}$ NMR (400 MHz, $\left.\mathbf{C D C l}_{3}\right) \delta 7.43-7.30$ (m, 5H), 4.20 (d, $J=5.6 \mathrm{~Hz}, 1 \mathrm{H}), 3.03(\mathrm{dd}, J=10.8,5.6 \mathrm{~Hz}, 1 \mathrm{H}), 2.27-2.18(\mathrm{~m}, 1 \mathrm{H}), 1.58-1.09(\mathrm{~m}, 22 \mathrm{H}) ;{ }^{13} \mathbf{C}$ NMR (100 MHz, $\left.\mathbf{C D C l}_{3}\right) \delta 136.7,129.2,128.8,128.6,112.4,112.0,49.3,37.3,28.0,26.4,25.6,25.5,25.4,25.2,23.3,23.1$, $22.5,22.4,21.1,20.8$. The spectral data matched those reported in the literature. ${ }^{9}$<smiles>CCC(C)(C)C(c1ccccc1)C(C#N)C#N</smiles>

\section{2-(2,2-Dimethyl-1-phenylbutyl)malononitrile (7) (containing regio- and diastereoisomers)}

General Procedure 1 with isopentane $(69.8 \mu \mathrm{L}, 0.60 \mathrm{mmol}, 3.0$ equiv) and benzalmalononitrile (30.8 $\mathrm{mg}, 0.20$ mmol): colorless oil, 99\% yield (47.9 mg, C1:C2 = 5.3:1, d.r. $(\mathrm{C} 2)=1.1: 1)$. Regioisomers could not be separated. ${ }^{1} \mathbf{H}$ NMR (400 MHz, $\left.\mathbf{C D C l}_{3}\right) \delta(\mathrm{C} 1$ product) 7.44-7.29 (m, 5H), $4.21(\mathrm{~d}, J=5.2 \mathrm{~Hz}, 1 \mathrm{H}), 3.07(\mathrm{~d}$, $J=5.2 \mathrm{~Hz}, 1 \mathrm{H}), 1.39(\mathrm{dq}, J=7.2 \mathrm{~Hz}, 2 \mathrm{H}), 1.12(\mathrm{~s}, 3 \mathrm{H}), 0.99(\mathrm{~s}, 3 \mathrm{H}), 0.89$ (t, $J=7.2 \mathrm{~Hz}, 3 \mathrm{H}) ; \delta(\mathrm{C} 2$ product) 7.44-7.29 (m, 5H), 4.15 (d, $J=3.6 \mathrm{~Hz}, 0.5 \mathrm{H}), 4.14(\mathrm{~d}, J=2.4 \mathrm{~Hz}, 0.5 \mathrm{~Hz}), 3.04(\mathrm{dd}, J=10.4,6.0 \mathrm{~Hz}, 0.5 \mathrm{H})$, $2.96(\mathrm{dd}, J=11.6,4.0 \mathrm{~Hz}, 0.5 \mathrm{H}), 2.24-2.12(\mathrm{~m}, 1 \mathrm{H}), 1.07(\mathrm{~d}, J=6.4 \mathrm{~Hz}, 1.5 \mathrm{H}), 1.01(\mathrm{~d}, J=6.4 \mathrm{~Hz}, 1.5 \mathrm{H})$, $0.88(\mathrm{~d}, J=2.5 \mathrm{~Hz}, 1.5 \mathrm{H}), 0.86(\mathrm{~d}, J=2.5 \mathrm{~Hz}, 1.5 \mathrm{H}), 0.88-0.85(\mathrm{~m}, 1 \mathrm{H}), 0.67(\mathrm{~d}, J=2,0 \mathrm{~Hz}, 1.5 \mathrm{H}), 0.66(\mathrm{~d}$, $J=2.0 \mathrm{~Hz}, 1.5 \mathrm{H}) ;{ }^{13} \mathbf{C} \mathbf{N M R}\left(\mathbf{1 0 0} \mathbf{~ M H z}, \mathbf{C D C l}_{3}\right) \delta$ (C1 product) 136.1, 129.7, 128.8, 128.7, 113.5, 113.3, 55.1, $37.5,33.5,24.9,24.8,24.6,8.3$. The spectral data matched those reported in the literature. ${ }^{10}$ 
<smiles>N#CC(C#N)C(Cc1ccccc1)c1ccccc1</smiles>

\section{2-(1,2-Diphenylethyl)malononitrile (8) [CAS: 61668-45-9]}

General Procedure 1 with toluene (106 $\mu \mathrm{L}, 1.0 \mathrm{mmol}, 5.0$ equiv) and benzalmalononitrile (30.8 $\mathrm{mg}, 0.20$ mmol): colorless oil, 86\% yield (42.2 mg). ${ }^{1} \mathbf{H}$ NMR (400 MHz, $\left.\mathbf{C D C l}_{3}\right) \delta$ 7.37-7.28 (m, 5H), 7.28-7.17 (m, 3), 7.13-7.09 (m, 2H), 3.77 (d, $J=5.2 \mathrm{~Hz}, 1 \mathrm{H}), 3.38$ (ddd, $J=8.0,7.6,5.6 \mathrm{~Hz}, 1 \mathrm{H}), 3.24-3.14(\mathrm{~m}, 2 \mathrm{H}) ;{ }^{13} \mathbf{C}$ NMR (100 MHz, $\left.\mathbf{C D C l}_{3}\right) \delta$ 136.8, 136.5, 129.30, 129.27, 129.2, 129.0, 128.1, 127.7, 112.2, 111.6, 48.5, 38.6, 28.6. The spectral data matched those reported in the literature. ${ }^{11}$

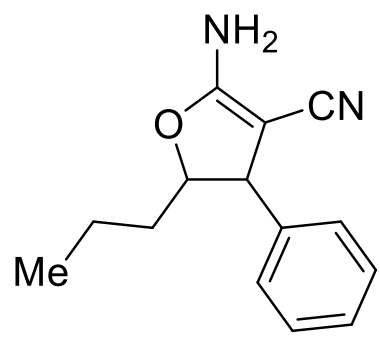

\section{2-Amino-4-phenyl-5-propyl-4,5-dihydrofuran-3-carbonitrile (9')}

General Procedure 1 with 1-butanol $(55.0 \mu \mathrm{L}, 0.60 \mathrm{mmol}, 3.0$ equiv) and benzalmalononitrile (30.8 $\mathrm{mg}, 0.20$ mmol): colorless solid, 92\% yield $(42.1 \mathrm{mg}$, d.r. $=1.0: 1)$. The corresponding product 9 was cyclized during silica-gel column chromatography to afford 9' (d.r. = 1.6:1): $\left.{ }^{1} \mathbf{H} \mathbf{~ N M R ~ ( 4 0 0 ~} \mathbf{M H z}, \mathbf{C D C l}_{3}\right) \delta\left(\mathbf{9}^{\prime}\right.$, mixture of diastereomers) 7.37-7.31 (m, 2H), 7.29-7.24 (m, 2.4H), 7.15-7.11 (m, 0.6H), 4.94-4.78 (m, 2.4H), 4.40 (ddd, $J=7.6,6.4,4.8 \mathrm{~Hz}, 0.6 \mathrm{H}), 4.20(\mathrm{~d}, J=8.8 \mathrm{~Hz}, 0.4 \mathrm{H}), 3.95(\mathrm{~d}, J=6.4 \mathrm{~Hz}, 0.6 \mathrm{H}), 1.83-1.64(\mathrm{~m}, 1.4 \mathrm{H})$, $1.53-1.31(\mathrm{~m}, 1.6 \mathrm{H}), 1.29-1.00(\mathrm{~m}, 1 \mathrm{H}), 0.92(\mathrm{t}, J=6.8 \mathrm{~Hz}, 1.8 \mathrm{H}), 0.78(\mathrm{t}, J=6.4 \mathrm{~Hz}, 1.2 \mathrm{H}) ;{ }^{13} \mathbf{C} \mathbf{N M R}(\mathbf{1 0 0}$ $\left.\mathbf{M H z}, \mathbf{C D C l}_{3}\right) \delta$ (9', mixture of diastereomers) $168.0,167.4,141.8,138.4,129.0,128.8,128.5,127.63,127.60$, $127.5,119.4,119.3,97.9,92.2,87.8,57.0,53.8,51.0,37.1,33.3,19.5,18.5,13.9,13.8$. The spectral data matched those reported in the literature. ${ }^{8}$<smiles>CC1(C)OC(N)=C(C#N)C1c1ccccc1</smiles>

\section{2-Amino-5,5-dimethyl-4-phenyl-4,5-dihydrofuran-3-carbonitrile (10')}

General Procedure 1 with 2-propanol $(46.0 \mu \mathrm{L}, 0.60 \mathrm{mmol}, 3.0$ equiv) and benzalmalononitrile (30.8 $\mathrm{mg}, 0.20$ $\mathrm{mmol})$ : colorless solid, $96 \%$ yield $(41.0 \mathrm{mg})$. The corresponding product $\mathbf{1 0}$ was cyclized during silica-gel column chromatography to afford 10': ' ${ }^{1} \mathbf{H}$ NMR (400 $\left.\mathbf{M H z}, \mathbf{C D C l}_{\mathbf{3}}\right) \delta\left(\mathbf{1 0}{ }^{\prime}\right)$ 7,40-7.31 (m, 2H), 7.31-7.25 (m, 
1H), 7.22-7.17 (m, 2H), 4.81 (br s, 2H), 4.04 (s, 1H), 1.56 (s, 3H), 0.88 (s, 3H); ${ }^{13} \mathbf{C}$ NMR (100 MHz, CDCl $)$ $\delta$ (10') $166.8,138.7,128.6,128.4,127.7,119.6,91.6,57.5,29.2,24.4$. The spectral data matched those reported in the literature. ${ }^{8}$<smiles>CC(C)CC(=O)C(c1ccccc1)C(C#N)C#N</smiles>

\section{2-(4-Methyl-2-oxo-1-phenylpentyl)malononitrile (11) [CAS: 2370983-39-2]}

General Procedure 1 with isovaleraldehyde $(64.6 \mu \mathrm{L}, 0.60 \mathrm{mmol}, 3.0$ equiv) and benzalmalononitrile (30.8 mg, 0.20 mmol): colorless oil, 98\% yield (47.2 mg). ${ }^{1} \mathbf{H}$ NMR (400 MHz, $\left.\mathbf{C D C l}_{3}\right) \delta 7.48-7.43(\mathrm{~m}, 3 \mathrm{H})$, $7.27-7.22(\mathrm{~m}, 2 \mathrm{H}), 4.39$ (d, $J=9.6 \mathrm{~Hz}, 1 \mathrm{H}), 4.23$ (d, $9.6 \mathrm{~Hz}, 1 \mathrm{H}), 2.33$ (dd, $J=16.0,6.4 \mathrm{~Hz}, 1 \mathrm{H}), 2.25$ (dd, $J$ $=16.0,7.6 \mathrm{~Hz}, 1 \mathrm{H}), 2.22-2.11(\mathrm{~m}, 1 \mathrm{H}), 0.90(\mathrm{~d}, J=6,4 \mathrm{~Hz}, 3 \mathrm{H}), 0.79(\mathrm{~d}, J=6.4 \mathrm{~Hz}, 3 \mathrm{H}) ;{ }^{13} \mathbf{C} \mathbf{N M R}(\mathbf{1 0 0}$ MHz, $\left.\mathbf{C D C l}_{3}\right) \delta 203.6,131.3,130.2,130.1,128.8,112.1,111.5,58.8,49.9,25.6,24.7,22.6,22.1$. The spectral data matched those reported in the literature. ${ }^{12}$<smiles>N#CC(C#N)C(C(=O)c1ccccc1)c1ccccc1</smiles>

\section{2-(2-Oxo-1,2-diphenylethyl)malononitrile (12) [CAS: 312307-43-0]}

General Procedure 1 with benzaldehyde $(60.6 \mu \mathrm{L}, 0.60 \mathrm{mmol}, 3.0$ equiv) and benzalmalononitrile (30.8 $\mathrm{mg}$, $0.20 \mathrm{mmol}$ ): colorless oil, 98\% yield (47.2 mg). ${ }^{1} \mathbf{H}$ NMR (400 $\left.\mathbf{~ M H z , ~} \mathbf{C D C l}_{3}\right) \delta$ 7.91-7,87 (m, 2H), 7.56-7.51 (m, 2H), 7.44-7.33 (m, 6H), $5.12(\mathrm{~d}, J=8.0 \mathrm{~Hz}, 1 \mathrm{H}), 4.54(\mathrm{~d}, J=8.0 \mathrm{~Hz}, 1 \mathrm{H}) ;{ }^{13} \mathbf{C} \mathbf{~ N M R}\left(\mathbf{1 0 0} \mathbf{~ M H z}, \mathbf{C D C l}_{3}\right)$ $\delta 193.2,134.5,133.9,132.1,130.2,130.0,129.4,129.0,128.7,112.2,111.7,54.8,26.9$. The spectral data matched those reported in the literature. ${ }^{9}$<smiles>C/C=C/C(=O)C(c1ccccc1)C(C#N)C#N</smiles>

\section{(E)-2-(2-Oxo-1-phenylpent-3-en-1-yl)malononitrile (13)}

General Procedure 1 with $\mathbf{1 c}(6.4 \mathrm{mg}, 0.020 \mathrm{mmol}, 10 \mathrm{~mol} \%)$ instead of $\mathbf{1 h}$, crotonaldehyde $(82.8 \mu \mathrm{L}, 1.0$ mmol, 5.0 equiv) and benzalmalononitrile (30.8 mg, $0.20 \mathrm{mmol})$ : yellow oil, $73 \%$ yield $(32.7 \mathrm{mg}) .{ }^{1} \mathbf{H}$ NMR (400 MHz, $\left.\mathbf{C D C l}_{3}\right) \delta$ 7.47-7.43 (m, 3H), 7.30-7.25 (m, 2H), $7.06(\mathrm{dq}, J=16.0,7.2 \mathrm{~Hz}, 1 \mathrm{H}), 6.07(\mathrm{dq}, J=$ 
16.0, 1.6 Hz, 1H), $4.43(\mathrm{~d}, J=8.8 \mathrm{~Hz}, 1 \mathrm{H}), 4.38(\mathrm{~d}, J=8.8 \mathrm{~Hz}, 1 \mathrm{H}), 1.86(\mathrm{dq}, J=7.2,1.6 \mathrm{~Hz}) ;{ }^{13} \mathbf{C}$ NMR $(\mathbf{1 0 0}$ MHz, $\left.\mathbf{C D C l}_{3}\right) \delta 191.9,147.7,131.5,130.1$ (2C), 129.0, 128.3, 112.2, 111.6, 56.9, 26.0, 18.7; HRMS (ESI) Calcd. For $\mathrm{C}_{14} \mathrm{H}_{12} \mathrm{ON}_{2} \mathrm{Na}: 247.0842\left([\mathrm{M}+\mathrm{Na}]^{+}\right)$. Found: 248.0844 .<smiles>CCC(NC(=O)OC(C)(C)C)C(c1ccccc1)C(C#N)C#N</smiles>

\section{tert-Butyl (1,1-Dicyano-2-phenylhexan-3-yl)carbamate (14)}

General Procedure 1 with tert-butyl butylcarbamate $(95.5 \mathrm{mg}, 0.60 \mathrm{mmol}, 3.0$ equiv) and benzalmalononitrile (30.8 mg, $0.20 \mathrm{mmol}$ ): colorless oil, 99\% yield (65.0 mg, d.r. = 1.5:1). ${ }^{1} \mathbf{H} \mathbf{~ N M R ~ ( 4 0 0 ~ M H z , ~} \mathbf{C D C l}_{3}$ ) $\delta$ (mixture of diastereomers) 7.47-7.30 (m, 4.2H), 7.23-7.16 (m, 0.8H), 4.99-4.60 (m, 0.6H), 4.55-4.27 (m, 1H), 4.20-3.89 (m, 1.4H), $3.39(\mathrm{~d}, J=10.4 \mathrm{~Hz}, 0.4 \mathrm{H}), 3.33-2.98(\mathrm{~m}, 0.6 \mathrm{H}), 1.60-1.08(\mathrm{~m}, 2 \mathrm{H}), 1.53(\mathrm{~s}, 5.4 \mathrm{H})$, $1.50(\mathrm{~s}, 3.6 \mathrm{H}), 0.97(\mathrm{t}, J=7.2 \mathrm{~Hz}, 1.2 \mathrm{H}), 086(\mathrm{t}, J=7.2 \mathrm{~Hz}, 1.8 \mathrm{H}) ;{ }^{13} \mathbf{C} \mathbf{~ N M R}\left(\mathbf{1 0 0} \mathbf{M H z}, \mathbf{C D C l}_{3}\right) \delta$ (mixture of diastereomers) 156.4, 156.2, 135.6, 133.4, 129.5 (2C), 129.3, 129.2, 128.9, 128.4, 113.0, 112.8, 112.3, 112.0, 80.8, 80.6, 54.9, 53.4, 51.9, 51.2, 28.4(2C), 27.5, 27.3, 26.9, 25.6, 10.8, 10.2; HRMS (ESI) Calcd. For $\mathrm{C}_{18} \mathrm{H}_{23} \mathrm{O}_{2} \mathrm{~N}_{3} \mathrm{Na}: 336.1693\left([\mathrm{M}+\mathrm{Na}]^{+}\right)$. Found: 336.1687 .<smiles>COC(=O)CC(C(=O)OC)C1CCCCC1</smiles>

\section{Dimethyl 2-Cyclohexylsuccinate (15) [CAS: 40745-20-8]}

General Procedure 1 with cyclohexane $(64.7 \mu \mathrm{L}, 0.60 \mathrm{mmol}, 3.0$ equiv) and dimethyl fumarate $(28.8 \mathrm{mg}, 0.20$ mmol): colorless oil, 83\% yield (38.0 mg). ${ }^{1} \mathbf{H}$ NMR (400 MHz, $\mathbf{C D C l}_{3}$ ) $\delta 3.69$ (s, 3H), 3.67 (s, 3H), 2.77-2.67 $(\mathrm{m}, 2 \mathrm{H}), 2.46(\mathrm{dt}, J=13.2,9.2 \mathrm{~Hz}, 1 \mathrm{H}), 1.78-1.70(\mathrm{~m}, 2 \mathrm{H}), 1.70-1.55(\mathrm{~m}, 4 \mathrm{H}), 1.31-0.97(\mathrm{~m}, 5 \mathrm{H}) ;{ }^{13} \mathbf{C}$ NMR (100 MHz, $\left.\mathbf{C D C l}_{3}\right) \delta 175.1,173.1,51.9,51.7,47.1,40.1,33.4,30.7,30.3,26.4(2 \mathrm{C}), 26.2$. The spectral data matched those reported in the literature. ${ }^{13}$ 
<smiles>CCOC(=O)C(C(=O)OCC)C(c1ccccc1)C1CCCCC1</smiles>

\section{Diethyl 2-(Cyclohexyl(phenyl)methyl)malonate (16) [CAS: 203565-36-0]}

General Procedure 1 with cyclohexane $(64.7 \mu \mathrm{L}, 0.60 \mathrm{mmol}, 3.0$ equiv) and diethyl benzylidenemalonate (45.1 mg, $0.20 \mathrm{mmol})$ : colorless oil, 47\% yield (30.5 mg). ${ }^{1} \mathbf{H}$ NMR (400 MHz, $\left.\mathbf{C D C l}_{3}\right) \delta$ 7.27-7.12 (m, 5H), $4.30-4.17(\mathrm{~m}, 2 \mathrm{H}), 3.97(\mathrm{~d}, J=10.8 \mathrm{~Hz}, 1 \mathrm{H}), 3.91-3.79(\mathrm{~m}, 2 \mathrm{H}), 3.37(\mathrm{dd}, J=10.8,4.0 \mathrm{~Hz}, 1 \mathrm{H}), 1.72-1.52$ $(\mathrm{m}, 6 \mathrm{H}), 1.32-1.06(\mathrm{~m}, 2 \mathrm{H}), 1.29(\mathrm{t}, J=6.8 \mathrm{~Hz}, 3 \mathrm{H}), 1.00-0.76(\mathrm{~m}, 3 \mathrm{H}), 0.91(\mathrm{t}, J=6.8 \mathrm{~Hz}, 3 \mathrm{H}) ;{ }^{13} \mathbf{C} \mathbf{~ N M R}$ (100 MHz, $\left.\mathbf{C D C l}_{3}\right) \delta 169.0,168.2,139.1,129.6,127.8,126.8,61.6,61.2,55.5,51.2,41.0,32.2,28.5,26.8$, 26.6, 26.3, 14.2, 13.8. The spectral data matched those reported in the literature. ${ }^{14}$<smiles>O=S(=O)(Oc1ccccc1)C(CC1CCCCC1)S(=O)(=O)c1ccccc1</smiles>

\section{(2-Cyclohexylethane-1,1-diyldisulfonyl)dibenzene (17) [CAS: 1802633-74-4]}

General Procedure 1 with cyclohexane $(64.7 \mu \mathrm{L}, 0.60 \mathrm{mmol}, 3.0$ equiv) and 1,1-bis(phenylsulfonyl)ethylene (61.7 mg, $0.20 \mathrm{mmol})$ : colorless solid, 77\% yield (60.5 mg). ${ }^{1} \mathbf{H}$ NMR (400 MHz, $\left.\mathbf{C D C l}_{3}\right) \delta 7.95$ (d, $J=7.6$ $\mathrm{Hz}, 4 \mathrm{H}), 7.69(\mathrm{t}, J=7.2 \mathrm{~Hz}, 2 \mathrm{H}), 7.57(\mathrm{t}, J=7.6 \mathrm{~Hz}, 4 \mathrm{H}), 4.46(\mathrm{t}, J=6.0 \mathrm{~Hz}, 1 \mathrm{H}), 2.00$ (dd, $J=7.2,5.6 \mathrm{~Hz}$, 2H), 1.70-1.54 (m, 5H), 1.52-1.39 (m, 1H), 1.20-1.01 (m, 3H), 0.83-0.70 (m, 2H); ${ }^{13} \mathbf{C}$ NMR (100 MHz, $\left.\mathbf{C D C l}_{3}\right) \delta 138.0,134.6,129.7,129.2,81.4,35.7,32.7,32.6,26.2,25.8$. The spectral data matched those reported in the literature. ${ }^{15}$<smiles>CCOC(=O)C1C(=O)Oc2ccccc2C1C1CCCCC1</smiles>

\section{Ethyl 4-Cyclohexyl-2-oxochromane-3-carboxylate (18) [CAS: 2624048-46-8]}

General Procedure 1 with cyclohexane $(64.7 \mu \mathrm{L}, 0.60 \mathrm{mmol}, 3.0$ equiv) and ethyl coumarin-3-carboxylate (43.6 mg, $0.20 \mathrm{mmol}$ ): colorless oil, $81 \%$ yield (49.0 mg, d.r. $>19: 1) .{ }^{1} \mathbf{H}$ NMR (400 $\left.\mathbf{~ M H z , ~} \mathbf{C D C l}_{3}\right) \delta$ 7.30-7.22 (m, 1H), 7.15-7.05 (m, 3H) 4.11-3.92 (m, 2H), 3.93 (d, $J=1.6 \mathrm{~Hz}, 1 \mathrm{H}), 3.11(\mathrm{dd}, J=8.0,1.2 \mathrm{~Hz}$, 1H), 1.90-1.55 (m, 5H), 1.48-1.36 (m, 1H), 1.22-1.08 (m, 3H), 1.06-0.82 (m, 2H), 0.99 (t, J= 7.2 Hz, 3H); 
${ }^{13}$ C NMR (100 MHz, CDCl $) \delta$ 167.8, 165.3, 151.2, 130.0, 128.8, 124.4, 123.3, 117.0, 62.2, 49.9, 46.2, 41.3, $30.7,30.1,26.2,26.1,13.9$ (1 aliphatic carbon peak was not found probably due to overlapping). The spectral data matched those reported in the literature. ${ }^{14}$<smiles>O=C(CCC1CCCCC1)OCc1ccccc1</smiles>

\section{Benzyl 3-Cyclohexylpropanoate (20) [CAS: 501357-36-4]}

General Procedure 2 with $\mathbf{1 h}(6.8 \mathrm{mg}, 0.020 \mathrm{mmol}, 10 \mathrm{~mol} \%)$ and cyclohexane $(64.7 \mu \mathrm{L}, 0.60 \mathrm{mmol}, 3.0$ equiv). Benzyl alcohol (41.1 $\mu \mathrm{L}, 0.40 \mathrm{mmol})$ was used for transesterification: colorless oil, 85\% yield (41.9 mg). ${ }^{1}$ H NMR (400 MHz, $\left.\mathbf{C D C l}_{3}\right) \delta 7.40-7.30(\mathrm{~m}, 5 \mathrm{H}), 5.11(\mathrm{~s}, 2 \mathrm{H}), 2.36(\mathrm{t}, J=8.0 \mathrm{~Hz}, 2 \mathrm{H}), 1.72-1.59$ (m, 5H), 1,57-1.50 (m, 2H), 1.29-1.07 (m, 4H), 0.94-0.81 (m, 2H); ${ }^{13} \mathbf{C}$ NMR (100 MHz, CDCl $) \delta$ 174.1, 136.3, $128.7,128.31,128.28,66.2,37.3,33.1,32.5,32.1,26.7,26.3$. The spectral data matched those reported in the literature. ${ }^{14}$<smiles>O=C(CCC1CCCCC1)NCc1ccccc1</smiles>

\section{$N$-Benzyl-3-cyclohexylpropanamide (21) [CAS: 351188-68-6]}

General Procedure 2 with $\mathbf{1 h}(6.8 \mathrm{mg}, 0.020 \mathrm{mmol}, 10 \mathrm{~mol} \%)$ and cyclohexane $(64.7 \mu \mathrm{L}, 0.60 \mathrm{mmol}, 3.0$ equiv). Benzylamine ( $43.7 \mu \mathrm{L}, 0.40 \mathrm{mmol}, 2.0$ equiv) was used for transamidation: colorless oil, $85 \%$ yield (41.9 mg). ${ }^{1} \mathbf{H}$ NMR (400 MHz, $\left.\mathbf{C D C l}_{3}\right) \delta 7.36-7.29$ (m, 2H), 7.29-7.21 (m, 3H), 5.90 (br s, 1H), 4.41 (d, $J$ $=5.6 \mathrm{~Hz}, 2 \mathrm{H}), 2.21(\mathrm{t}, J=8.0 \mathrm{~Hz}, 2 \mathrm{H}), 1.75-1.60(\mathrm{~m}, 5 \mathrm{H}), 1.57-1.51(\mathrm{~m}, 2 \mathrm{H}), 1.30-1.09(\mathrm{~m}, 4 \mathrm{H}), 0.94-0.82$ $(\mathrm{m}, 2 \mathrm{H}) ;{ }^{13} \mathbf{C}$ NMR (100 MHz, $\left.\mathbf{C D C l}_{3}\right) \delta$ 173.4, 138.6, 128.8, 127.9, 127.6, 43.7, 37.5, 34.4, 33.3, 33.2, 26.6, 26.3; HRMS (ESI) Calcd. For $\mathrm{C}_{16} \mathrm{H}_{23} \mathrm{ONNa} 268.1683\left([\mathrm{M}+\mathrm{Na}]^{+}\right)$. Found: 268.1674.

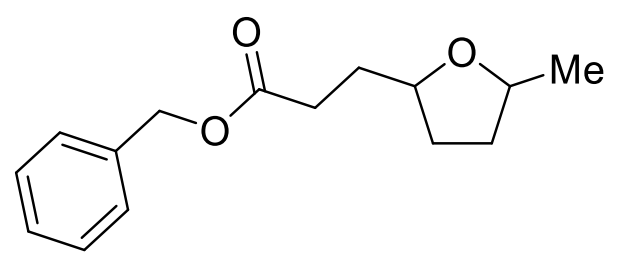

\section{Benzyl 3-(5-Methyltetrahydrofuran-2-yl)propanoate (22)}

General Procedure 2 with $\mathbf{1 h}(6.8 \mathrm{mg}, 0.020 \mathrm{mmol}, 10 \mathrm{~mol} \%)$ and 2-methyltetrahydrofuran $(401 \mu \mathrm{L}, 4.0 \mathrm{mmol}$, 20 equiv). Benzyl alcohol (41.1 $\mu \mathrm{L}, 0.40 \mathrm{mmol}$ ) was used for transesterification: colorless oil, $99 \%$ yield (41.9 mg, r.r. >19:1, d.r. = 1.7:1). ${ }^{1} \mathbf{H}$ NMR (400 $\left.\mathbf{M H z}, \mathbf{C D C l}_{3}\right) \delta$ (mixture of diastereomers) 7.40-7.28 (m, 5H), 5,11 (s, 2H), 4.10-3.89 (m, 0.9H), 3.87-3.72 (m, 1.1H), 2.56-2.36 (m, 2H), 2.09-1.79 (m, 4H), 1.77-1.61 (m, 0.9H), 1.57-1.39 (m, 1.1H), 1.22-1.19 (m, 1.4H), $1.18(\mathrm{~s}, 1.6 \mathrm{H}) ;{ }^{13} \mathbf{C}$ NMR (100 MHz, $\left.\mathbf{C D C l}_{3}\right) \delta$ (mixture of 
diastereomers) 173.9, 173.5, 136.2, 128.6, 128.3, 128.2, 81.7, 78.3, 77.7, 75.5, 74.6, 67.4, 66.3, 66.2, 37.0, 35.6, 33.9, 32.9, 32.1, 31.22, 31.18, 31.14, 31.09, 29.8, 26.1, 25.8, 21.4, 21.3; HRMS (ESI) Calcd. For $\mathrm{C}_{15} \mathrm{H}_{20} \mathrm{O}_{3} \mathrm{Na}: 271.1309\left([\mathrm{M}+\mathrm{Na}]^{+}\right)$. Found: 271.1305. The spectral data matched those reported in the literature. ${ }^{16}$

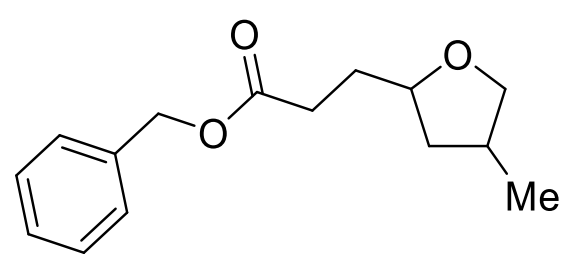

\section{Benzyl 3-(4-Methyltetrahydrofuran-2-yl)propanoate (23)}

General Procedure 2 with $\mathbf{1 h}(6.8 \mathrm{mg}, 0.020 \mathrm{mmol}, 10 \mathrm{~mol} \%)$ and 3-methyltetrahydrofuran $(396 \mu \mathrm{L}, 4.0 \mathrm{mmol}$, 20 equiv). Benzyl alcohol ( $41.1 \mu \mathrm{L}, 0.40 \mathrm{mmol})$ was used for transesterification: colorless oil, $81 \%$ yield (40.0 $\mathrm{mg}$, r.r. $=10: 1$ (ratio of major isomer relative to all minor isomers), d.r. $=1.7: 1$ ). Major regioisomer was separated by PLC. ${ }^{1} \mathbf{H}$ NMR (400 $\left.\mathbf{M H z}, \mathbf{C D C l}_{3}\right) \delta$ (major regioisomer, mixture of diastereomers) 7.78-7.29 $(\mathrm{m}, 5 \mathrm{H}), 5.12(\mathrm{~s}, 2 \mathrm{H}), 3.99-3.76(\mathrm{~m}, 2 \mathrm{H}), 3.32(\mathrm{td}, \mathrm{J}=10.0,3.2 \mathrm{~Hz}, 0.6 \mathrm{H}), 3.24(\mathrm{t}, J=10.0 \mathrm{~Hz}, 0.4 \mathrm{H})$, 2.60-2.38 (m, 2H), 2.35-2.03 (m, 1H), 1.98-1.65 (m, 3H), 1.62-1.47 (m, 1H), 1.04-0.99 (m, 3H); ${ }^{13} \mathbf{C ~ N M R}$ $\left(\mathbf{1 0 0} \mathbf{~ M H z}, \mathbf{C D C l}_{3}\right) \delta$ (major regioisomer, mixture of diastereomers) 173.7, 173.6, 136.3, 136.2, 128.6(2C), 128.3(4C), 84.9, 77.8, 75.0, 66.9, 66.29, 66.27, 39.5, 39.0, 34.8, 33.4, 31.3, 31.22, 31.18, 29.4, 18.1, 17.2; HRMS (ESI) Calcd. For $\mathrm{C}_{15} \mathrm{H}_{20} \mathrm{O}_{3} \mathrm{Na}: 271.1309\left([\mathrm{M}+\mathrm{Na}]^{+}\right)$. Found: 271.1305.<smiles>CC(OCC(c1ccccc1)C(C#N)C#N)c1ccccc1</smiles>

\section{2-(1-Phenyl-3-(1-phenylethoxy)propyl)malononitrile (24)}

General Procedure 1 with (1-methoxyethyl)benzene (136 mg, $1.0 \mathrm{mmol}, 5.0$ equiv) and benzalmalononitrile (30.8 mg, $0.20 \mathrm{mmol})$ : colorless oil, 80\% yield (46.3 mg, r.r. $>19: 1$, d.r. $=1.0: 1)$. $\left.{ }^{1} \mathbf{H} \mathbf{~ N M R ~ ( 4 0 0 ~} \mathbf{~ M H z}, \mathbf{C D C l}_{3}\right)$ $\delta$ (mixture of diastereomers) 7.39-7.23 (m, 10H), 4.51-4.43 (m, 2H), 3.75-3.62 (m, 2H), $3.46(\mathrm{dt}, J=10.4$, $4.8 \mathrm{~Hz}, 0.5 \mathrm{H}), 3.39$ (dt, $J=10.0,5.2 \mathrm{~Hz}, 0.5 \mathrm{H}), 1.51(\mathrm{~s}, 1.5 \mathrm{H}), 1.45(\mathrm{~s}, 1.5 \mathrm{H}) ;{ }^{13} \mathbf{C} \mathbf{~ N M R}\left(\mathbf{1 0 0} \mathbf{M H z}, \mathbf{C D C l}_{\mathbf{3}}\right)$ $\delta$ (mixture of diastereomers) 142.5, 142.3, 134.7, 134.6, 129.28(2C), 129.23(2C), 128.83, 128.79, 128.3, 128.23, 128.16, 128.1, 126.3, 126.2, 112.4, 112.3, 111.8, 111.7, 79.6, 79.4, 68.0, 67.7, 46.7, 46.6, 26.5, 26.4, 23.8, 23.7; HRMS (ESI) Calcd. For $\mathrm{C}_{19} \mathrm{H}_{18} \mathrm{ON}_{2} \mathrm{Na}$ : 313.1314 ([M + Na $\left.]^{+}\right)$. Found: 313.1311.

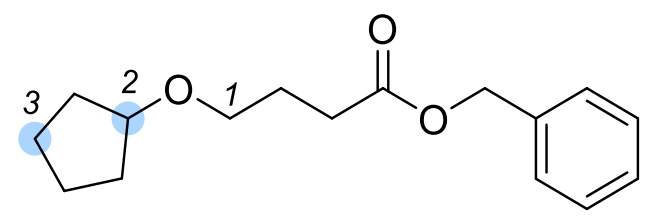

Benzyl 4-(Cyclopentyloxy)butanoate (25) 
General Procedure 2 with $1 \mathbf{i}(9.5 \mathrm{mg}, 0.020 \mathrm{mmol}, 10 \mathrm{~mol} \%$ ) and cyclopentyl methyl ether (466 $\mu \mathrm{L}, 4.0 \mathrm{mmol}$, 20 equiv). Benzyl alcohol (41.1 $\mu \mathrm{L}, 0.40 \mathrm{mmol})$ was used for transesterification: colorless oil, $90 \%$ yield (47.2 mg, C1:C2:C3 = 18:1:4.0). C1 product was separated by PLC: ${ }^{1} \mathbf{H}$ NMR (400 MHz, $\left.\mathbf{C D C l}_{3}\right) \delta(\mathrm{C} 1$ product) 7.40-7.29 (m, 5H), $5.11(\mathrm{~s}, 2 \mathrm{H}), 3.85-3.83(\mathrm{~m}, 1 \mathrm{H}), 3.39(\mathrm{t}, J=6.0 \mathrm{~Hz}, 2 \mathrm{H}), 2.44(\mathrm{t}, J=7.2 \mathrm{~Hz}, 2 \mathrm{H}), 1.89$ (tt, $J=7.2,6.0 \mathrm{~Hz}, 2 \mathrm{H}), 1.73-1.62(\mathrm{~m}, 4 \mathrm{H}), 1.62-1.54(\mathrm{~m}, 2 \mathrm{H}), 1.53-1.43(\mathrm{~m}, 2 \mathrm{H}) ;{ }^{13} \mathbf{C} \mathbf{~ N M R}\left(\mathbf{1 0 0} \mathbf{~ M H z}, \mathbf{C D C l}_{3}\right)$ $\delta$ (C1 product) 173.5, 136.2, 128.7, 128.3(2C), 81.4, 67.5, 66.2, 32.4, 31.4, 25.5, 23.6; HRMS (ESI) Calcd. For $\mathrm{C}_{16} \mathrm{H}_{22} \mathrm{O}_{3} \mathrm{Na}$ : $285.1464\left([\mathrm{M}+\mathrm{Na}]^{+}\right)$. Found: 285.1461.<smiles>CCC(C)OCCCC(=O)OCc1ccccc1</smiles>

Benzyl 4-(sec-Butoxy)butanoate (26)

General Procedure 2 with $1 \mathbf{i}(9.5 \mathrm{mg}, 0.020 \mathrm{mmol}, 10 \mathrm{~mol} \%$ ) and sec-butyl methyl ether (476 $\mu \mathrm{L}, 4.0 \mathrm{mmol}$, 20 equiv). Benzyl alcohol (41.1 $\mu \mathrm{L}, 0.40 \mathrm{mmol}$ ) was used for transesterification: colorless oil, $82 \%$ yield (41.1 mg, r.r. $=18: 1)$. Major regioisomer was separated by PLC. ${ }^{1} \mathbf{H}$ NMR (400 $\left.\mathbf{M H z}, \mathbf{C D C l}_{3}\right) \delta$ (major regioisomer) $7.38-7.29(\mathrm{~m}, 5 \mathrm{H}), 5.12(\mathrm{~s}, 2 \mathrm{H}), 3.49(\mathrm{dt}, J=9.2,6.0 \mathrm{~Hz}, 1 \mathrm{H}), 3.37(\mathrm{dt}, J=9.2,6.0 \mathrm{~Hz}, 1 \mathrm{H}), 3.32-3.23(\mathrm{~m}$, $1 \mathrm{H}), 2.47(\mathrm{t}, J=8.0 \mathrm{~Hz}, 2 \mathrm{H}), 1.90(\mathrm{tt}, J=6.4,6.4 \mathrm{~Hz}, 2 \mathrm{H}), 1.56-1.45(\mathrm{~m}, 1 \mathrm{H}), 1.45-1.34(\mathrm{~m}, 1 \mathrm{H}), 1.09$ (d, $J$ $=6.0 \mathrm{~Hz}, 3 \mathrm{H}), 0.87(\mathrm{t}, J=7.2 \mathrm{~Hz}, 3 \mathrm{H}) ;{ }^{13} \mathbf{C} \mathbf{~ N M R}\left(\mathbf{1 0 0} \mathbf{~ M H z}, \mathbf{C D C l}_{3}\right) \delta$ (major regioisomer) 173.6, 136.2, 128.7, 128.3(2C), 76.8, 67.2, 66.2, 31.3, 29.3, 25.5, 19.3, 9.9; HRMS (ESI) Calcd. For $\mathrm{C}_{15} \mathrm{H}_{22} \mathrm{O}_{3} \mathrm{Na}$ : 273.1465 $\left([\mathrm{M}+\mathrm{Na}]^{+}\right)$. Found: 273.1461 .

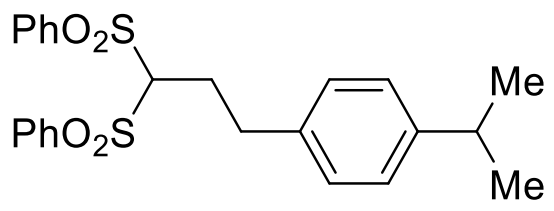

\section{(3-(4-Isopropylphenyl)propane-1,1-diyldisulfonyl)dibenzene (27)}

General Procedure 1 with $\mathbf{1 i}$ (9.5 mg, $0.020 \mathrm{mmol}, 10 \mathrm{~mol} \%$ ) instead of $\mathbf{1 h}, p$-cymene (312 $\mu \mathrm{L}, 2.0 \mathrm{mmol}, 10$ equiv) and 1,1-bis(phenylsulfonyl)ethylene $(61.7 \mathrm{mg}, 0.20 \mathrm{mmol})$ : colorless oil, $60 \%$ yield $(53.0 \mathrm{mg}$, r.r. $=$ 12:1). Major regioisomer was separated by PLC. ${ }^{1} \mathbf{H}$ NMR (400 $\left.\mathbf{~ M H z , ~} \mathbf{C D C l}_{3}\right) \delta$ (major regioisomer) 7.90-7.80 (m, 4H), 7.69-7.65 (m, 2H), 7.55-7.51 (m, 4H), 7.08 (d, J=8.0 Hz, 2H), $6.94(\mathrm{~d}, J=8.0 \mathrm{~Hz}, 2 \mathrm{H})$, $4.36(\mathrm{t}, J=5.6 \mathrm{~Hz}, 1 \mathrm{H}), 2.92-2.81(\mathrm{~m}, 3 \mathrm{H}), 2.47(\mathrm{q}, J=6.8 \mathrm{~Hz}, 2 \mathrm{H}), 1.24(\mathrm{~d}, J=6.8 \mathrm{~Hz}, 6 \mathrm{H}) ;{ }^{13} \mathbf{C} \mathbf{N M R}(\mathbf{1 0 0}$ MHz, $\mathbf{C D C l}_{3}$ ) $\delta$ (major regioisomer) 147.3, 138.0, 136.0, 135.0, 129.6, 129.2, 128.7, 126.9, 81.8, 33.8, 32.9, 27.1, 24.1; HRMS (ESI) Calcd. For $\mathrm{C}_{24} \mathrm{H}_{25} \mathrm{O}_{4} \mathrm{~S}_{2}: 441.1202$ ([M - H]-). Found: 441.1189. 
<smiles>CC(CCCC(C)(C)C(c1ccccc1)C(C#N)C#N)CCOC(=O)c1ccccc1</smiles>

\section{9,9-Dicyano-3,7,7-trimethyl-8-phenylnonyl benzoate (28)}

General Procedure 1 with 3,7-dimethyloctyl benzoate (262 mg, $1.0 \mathrm{mmol}, 5.0$ equiv) and benzalmalononitrile (30.8 mg, $0.20 \mathrm{mmol}$ ): colorless oil, $41 \%$ yield (34.0 mg, r.r. $>19: 1$, d.r. $\left.=1.0: 1) .{ }^{1} \mathbf{H} \mathbf{~ N M R ~ ( 4 0 0 ~} \mathbf{~ M H z}, \mathbf{C D C l}_{3}\right)$ $\delta$ (mixture of diastereomers) 8.06-8.02 (m, 2H), 7.58-7.54 (m, 1H), 7.46-7.42 (m, 2H), 7.40-7.35 (m, 5H), 4.41-4.29 (m, 2H), 4.20 (d, $J=5.6 \mathrm{~Hz}, 1 \mathrm{H}), 3.06(\mathrm{~d}, J=5.6 \mathrm{~Hz}, 1 \mathrm{H}), 1.82-1.73(\mathrm{~m}, 1 \mathrm{H}), 1.68-1.52(\mathrm{~m}, 3 \mathrm{H})$, $1.40-1.24(\mathrm{~m}, 5 \mathrm{H}), 1.132$ (s, 1.5H), 1.129 (s, 1.5H), 1.01 (s, 3H), 0.96 (d, $J=3.6 \mathrm{~Hz}, 1.5 \mathrm{H}), 0.95$ (d, $J=3.6$ $\mathrm{Hz}, 1.5 \mathrm{H}) ;{ }^{13} \mathbf{C}$ NMR (100 MHz, $\mathbf{C D C l}_{3}$ ) $\delta$ (mixture of diastereomers) 166.8, 136.1, 133.0, 130.6, 129.7, 128.9, 128.8, 128.5, 113.4, 113.2, 63.48, 63.45, 55.5, 41.4, 37.6, 37.5, 35.7, 30.0, 25.5, 25.2, 25.0, 21.2, 21.20, 21.17, 19.7(2C); HRMS (ESI) Calcd. For $\mathrm{C}_{27} \mathrm{H}_{32} \mathrm{O}_{2} \mathrm{~N}_{2} \mathrm{Na}$ : $439.2358\left([\mathrm{M}+\mathrm{Na}]^{+}\right)$. Found: 439.2356. The spectral data matched those reported in the literature. ${ }^{10}$<smiles>O=C1CCCC(CC(S(=O)(=O)c2ccccc2)S(=O)(=O)c2ccccc2)C1</smiles>

\section{3-(2,2-Bis(phenylsulfonyl)ethyl)cyclohexan-1-one (29)}

General Procedure 1 with cyclohexanone (207 $\mu \mathrm{L}, 2.0 \mathrm{mmol}, 10$ equiv) and 1,1-bis(phenylsulfonyl)ethylene (61.7 mg, $0.20 \mathrm{mmol})$ : colorless oil, $67 \%$ yield $(54.8 \mathrm{mg}$, r.r. $=9.2: 1)$. Major regioisomer was separated by PLC. ${ }^{1} \mathbf{H}$ NMR (400 MHz, $\left.\mathbf{C D C l}_{3}\right) \delta$ (major regioisomer) 7.95-7.91 (m, 4H), 7.74-7.70 (m, 2H), 7.61-7.57 (m, 4H), $4.40(\mathrm{t}, J=5.2 \mathrm{~Hz}, 1 \mathrm{H}), 2.40-2.31(\mathrm{~m}, 2 \mathrm{H}), 2.31-2.17(\mathrm{~m}, 2 \mathrm{H}), 2.12-1.98(\mathrm{~m}, 2 \mathrm{H}), 1.91-1.85$ (m, 2H), 1.66-1.53 (m, 2H), 1.35-1.23 (m, 1H); ${ }^{13} \mathbf{C ~ N M R ~ ( 1 0 0 ~ M H z , ~} \mathbf{C D C l}_{3}$ ) $\delta$ (major regioisomer) 209.6, 137.8, 137.4, 135.0, 134.9, 129.8, 129.7, 129.39, 129.37, 81.1, 47.1, 41.1, 37.1, 31.9, 31.8, 24.6; HRMS (ESI) Calcd. For $\mathrm{C}_{20} \mathrm{H}_{21} \mathrm{O}_{5} \mathrm{~S}_{2}: 405.0838$ ([M - H] $\left.]^{-}\right)$. Found: 405.0825.

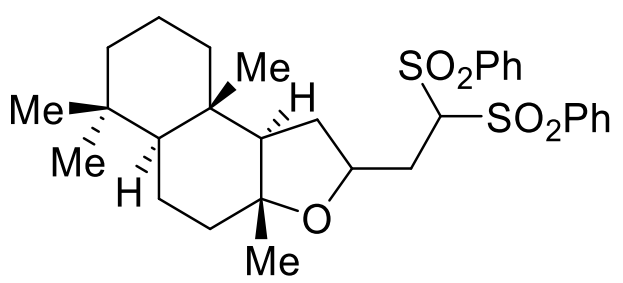

(3aR,5aS,9aS,9bR)-2-(2,2-Bis(phenylsulfonyl)ethyl)-3a,6,6,9a-tetramethyldodecahydronaphtho[2,1b]furan (30) [CAS: 2375385-63-8] 
General Procedure 1 with ambroxide (142 mg, $0.60 \mathrm{mmol}, 3.0$ equiv) and 1,1-bis(phenylsulfonyl)ethylene (61.7 mg, $0.20 \mathrm{mmol})$ : colorless solid, 78\% yield (85.0 mg, r.r. $>19: 1$, d.r. $=2.0: 1) .{ }^{1} \mathbf{H}$ NMR (400 MHz, $\left.\mathbf{C D C l}_{3}\right) \delta$ (mixture of diastereomers) 8.01-7.95 (m, 2H), 7.93-7.85 (m, 2H), 7.71-7.63 (m, 2H), 7.60-7.50 $(\mathrm{m}, 4 \mathrm{H}), 4.97-4.89(\mathrm{~m}, 1 \mathrm{H}), 4.35-4.20(\mathrm{~m}, 1 \mathrm{H}), 2.48-2.34(\mathrm{~m}, 1 \mathrm{H}), 2.33-2.24(\mathrm{~m}, 0.3 \mathrm{H}), 2.22-2.13(\mathrm{~m}, 0.7 \mathrm{H})$, 1.94-1.54 (m, 5H), 1.45-1.10 (m, 8H), 1.06-0.72 (m, 13H); ${ }^{13} \mathbf{C}$ NMR (100 MHz, $\left.\mathbf{C D C l}_{3}\right) \delta$ (mixture of diastereomers) 138.9, 138.7, 138.2, 137.8, 134.51, 134.47, 134.4(2C), 129.7, 129.64(2C), 129.56, 129.2(2C), 129.1(2C), 81.6, 81.1, 80.7, 79.6, 74.8, 71.7, 61.0, 58.5, 57.2, 57.0, 42.52, 42.45, 40.3, 40.0, 39.9, 39.8, 36.3, 36.0, 34.2, 33.7, 33.6, 33.5, 33.12, 33.09, 29.6, 28.9, 24.3, 21.9, 21.12, 21.10, 20.8, 20.5, 18.42, 18.38, 15.5, 15.1 ( 4 aliphatic carbon peaks were not found probably due to overlapping). The spectral data matched those reported in the literature. ${ }^{15}$

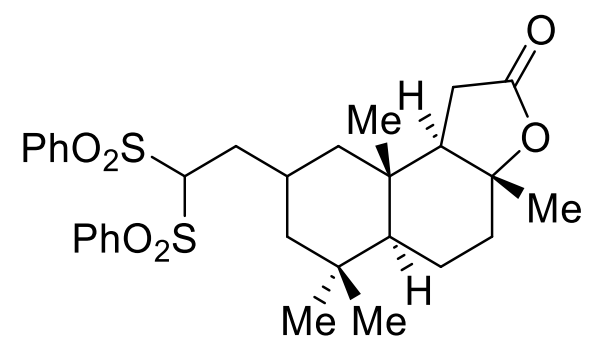

(3aR,5aS,9aS,9bR)-3a,6,6,9a-Tetramethyl-8-(2-((phenylperoxy)thio)-2-

(phenylsulfonyl)ethyl)decahydronaphtho[2,1-b]furan-2(1H)-one (31)

General Procedure 1 with sclareolide (150 mg, $0.60 \mathrm{mmol}, 3.0$ equiv) and 1,1-bis(phenylsulfonyl)ethylene (61.7 mg, $0.20 \mathrm{mmol})$ : colorless solid, 81\% yield (90.0 mg, r.r. > 19:1, d.r. >19:1). ${ }^{1}$ H NMR (400 MHz, CDCl $)$ ) $7.99-7.93(\mathrm{~m}, 4 \mathrm{H}), 7.75-7.69(\mathrm{~m}, 2 \mathrm{H}), 7.63-7.58(\mathrm{~m}, 4 \mathrm{H}), 4.43(\mathrm{t}, J=5.6 \mathrm{~Hz}, 1 \mathrm{H}), 2.38(\mathrm{t}, J=15.6$ $\mathrm{Hz}, 1 \mathrm{H}), 2.16(\mathrm{dd}, J=15.6,6.4 \mathrm{~Hz}, 1 \mathrm{H}), 2.06(\mathrm{dt}, J=12.4,3.2 \mathrm{~Hz}, 1 \mathrm{H}), 1.97$ (t, $J=5.6 \mathrm{~Hz}, 2 \mathrm{H}), 1.90-1.80$ $(\mathrm{m}, 3 \mathrm{H}), 1.73-1.60(\mathrm{~m}, 2 \mathrm{H}), 1.40-1.25(\mathrm{~m}, 2 \mathrm{H}), 1.31(\mathrm{~s}, 3 \mathrm{H}), 0.96-0.67(\mathrm{~m}, 2 \mathrm{H}), 0.85(\mathrm{~s}, 3 \mathrm{H}), 0.81(\mathrm{~s}, 3 \mathrm{H})$, $0.75(\mathrm{~s}, 3 \mathrm{H}), 0.55(\mathrm{t}, J=12.0 \mathrm{~Hz}, 1 \mathrm{H}) ;{ }^{13} \mathbf{C} \mathbf{N M R}\left(\mathbf{1 0 0} \mathbf{M H z}, \mathbf{C D C l}_{3}\right) \delta 176.5,138.1,137.7,134.8(2 \mathrm{C}), 129.8$, 129.6, 129.4, 129.3, 86.2, 81.3, 58.9, 56.6, 48.4, 45.6, 38.6, 36.6, 33.8, 33.1, 32.7, 28.7, 27.6, 21.7, 21.5, 20.5, 15.8; HRMS (ESI) Calcd. For $\mathrm{C}_{30} \mathrm{H}_{38} \mathrm{O}_{6} \mathrm{NaS}_{2}: 581.2002\left([\mathrm{M}+\mathrm{Na}]^{+}\right)$. Found: 581.2009.<smiles>COC(=O)CCC(C)(C)[C@@H]1CC[C@@H](C)C[C@H]1O</smiles>

\section{Methyl 4-((1S,2R,4R)-2-Hydroxy-4-methylcyclohexyl)-4-methylpentanoate (32)}

General Procedure 3 with $\mathbf{1 h}(6.8 \mathrm{mg}, 0.020 \mathrm{mmol}, 10 \mathrm{~mol} \%)$ and $l$-menthol (93.8 $\mathrm{mg}, 0.60 \mathrm{mmol}, 3.0$ equiv): colorless oil, 88\% yield (42.6 mg, r.r. = 10:1). Major regioisomer was separated by PLC. ${ }^{1}$ H NMR (400 MHz, $\left.\mathbf{C D C l}_{3}\right) \delta$ (major regioisomer) $3.67(\mathrm{~s}, 3 \mathrm{H}), 3.60-3.50(\mathrm{~m}, 1 \mathrm{H}), 2.38$ (ddd, $\left.J=16.4,11.2,6.0 \mathrm{~Hz}, 1 \mathrm{H}\right), 2.25$ (ddd, $J=16.4,11.2,6.4 \mathrm{~Hz}, 1 \mathrm{H}), 1.94-1.84(\mathrm{~m}, 2 \mathrm{H}), 1.73-1.62(\mathrm{~m}, 3 \mathrm{H}), 1.48-1.35(\mathrm{~m}, 1 \mathrm{H}), 1.13-1.05$ (m, 
1H), 1.04-0.75 (m, 4H), 1.00 (s, 3H), 0.94 (s, 3H), 0.89 (d, $J=6.8 \mathrm{~Hz}, 3 \mathrm{H}) ;{ }^{13} \mathbf{C}$ NMR (100 MHz, CDCl 3$) \delta$ (major regioisomer) 175.5, 73.0, 51.7, 50.5, 47.1, 37.0, 35.2, 35.1, 31.9, 29.4, 26.5, 26.2, 26.1, 22.1; HRMS (ESI) Calcd. For $\mathrm{C}_{14} \mathrm{H}_{26} \mathrm{O}_{3} \mathrm{Na}: 265.1774\left([\mathrm{M}+\mathrm{Na}]^{+}\right)$. Found: 265.1776.

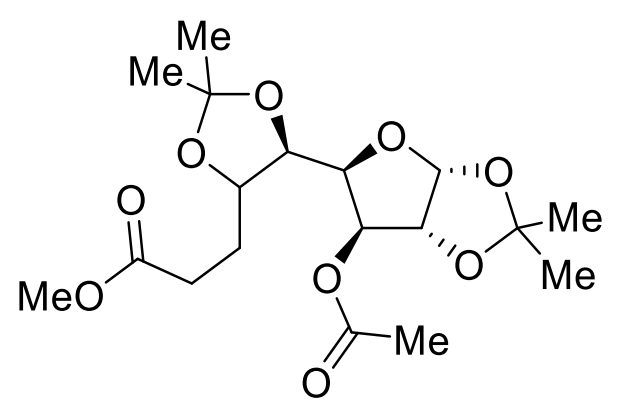

Methyl 3-((5R)-5-((3aR,5R,6S,6aR)-6-Acetoxy-2,2-dimethyltetrahydrofuro[2,3-d][1,3]dioxol-5-yl)-2,2dimethyl-1,3-dioxolan-4-yl)propanoate (33)

General Procedure 3 with $\mathbf{1 h}(6.8 \mathrm{mg}, 0.020 \mathrm{mmol}, 10 \mathrm{~mol} \%)$ and 3- $O$-acetyl-1,2:5,6-di- $O$-isopropylidene- $\alpha$ D-glucofuranose (181 mg, $0.60 \mathrm{mmol}, 3.0$ equiv): colorless oil, $77 \%$ yield $(60.0 \mathrm{mg}$, r.r. $>19: 1$, d.r. $=3.2: 1)$. Major diastereomer was separated by PLC. ${ }^{1} \mathbf{H}$ NMR $\left(400 \mathbf{~ M H z}, \mathbf{C D C l}_{3}\right) \delta$ (major diastereomer) $5.89(\mathrm{~d}, J=$ $11.2 \mathrm{~Hz}, 1 \mathrm{H}), 5.29(\mathrm{~d}, J=11.2 \mathrm{~Hz}, 1 \mathrm{H}), 4.49$ (d, $J=3.6 \mathrm{~Hz}, 1 \mathrm{H}), 4.16$ (dd, $J=12.8,3.6 \mathrm{~Hz}, 1 \mathrm{H}), 4.03(\mathrm{td}, J$ $=8.0,3.6 \mathrm{~Hz}, 1 \mathrm{H}), 3.78(\mathrm{dd}, J=8.8,7.2 \mathrm{~Hz}, 1 \mathrm{H}), 3.68(\mathrm{~s}, 3 \mathrm{H}), 2.60-2.40(\mathrm{~m}, 2 \mathrm{H}), 2.20-2.07(\mathrm{~m}, 1 \mathrm{H}), 2.10$ (s, 3H), 1.94-1.83 (m, 1H), $1.52(\mathrm{~s}, 3 \mathrm{H}), 1.35(\mathrm{~s}, 6 \mathrm{H}), 1.31(\mathrm{~s}, 3 \mathrm{H}) ;{ }^{13} \mathbf{C}$ NMR (100 MHz, CDCl $) \delta$ (major diastereomer) 173.9, 169.7, 112.3, 109.4, 105.3, 83.3, 80.5, 80.0, 76.4, 51.7, 30.7, 28.7, 27.3, 27.1 26.8, 26.2, 20.9 ( 1 peak was not found probably due to overlapping); HRMS (ESI) Calcd. For $\mathrm{C}_{18} \mathrm{H}_{28} \mathrm{O}_{9} \mathrm{Na}$ : 411.1626 $\left([\mathrm{M}+\mathrm{Na}]^{+}\right)$. Found: 411.1640 .

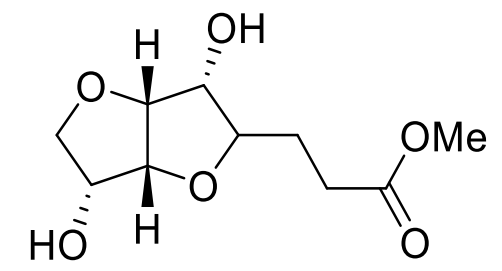

Methyl 3-((3R,3aR,6R,6aR)-3,6-Dihydroxyhexahydrofuro[3,2-b]furan-2-yl)propanoate (34)

General Procedure 3 with $\mathbf{1 h}(6.8 \mathrm{mg}, 0.020 \mathrm{mmol}, 10 \mathrm{~mol} \%)$ and isomannide (87.6 mg, $0.60 \mathrm{mmol}, 3.0$ equiv): colorless oil, 88\% yield (27.9 mg, r.r. $>19: 1$, d.r. $>19: 1) .{ }^{1} \mathbf{H}$ NMR (400 MHz, $\left.\mathbf{C D C l}_{3}\right) \delta 4.54(\mathrm{t}, J=$ $5.2 \mathrm{~Hz}, 1 \mathrm{H}), 4.47(\mathrm{t}, J=5.2 \mathrm{~Hz}, 1 \mathrm{H}), 4.35-4.27(\mathrm{~m}, 1 \mathrm{H}), 4.02(\mathrm{dd}, J=10.0,5.6 \mathrm{~Hz}, 1 \mathrm{H}), 3.85-3.79(\mathrm{~m}, 1 \mathrm{H})$, $3.69(\mathrm{~s}, 3 \mathrm{H}), 3.66-3.60(\mathrm{~m}, 2 \mathrm{H}), 2.74(\mathrm{~d}, J=6.8 \mathrm{~Hz}, 1 \mathrm{H}), 2.59(\mathrm{~d}, J=5.2 \mathrm{~Hz}, 1 \mathrm{H}), 2.50(\mathrm{t}, J=6.8 \mathrm{~Hz}, 2 \mathrm{H})$, 2.10-2.00 (m, 1H), 1.94-1.84 (m, 1H); ${ }^{13} \mathbf{C}$ NMR (100 MHz, $\left.\mathbf{C D C l}_{3}\right) \delta$ 173.8, 83.6, 81.9, 80.5, 77.4, 74.8, 72.7, 51.9, 30.5, 28.4; HRMS (ESI) Calcd. For $\mathrm{C}_{10} \mathrm{H}_{16} \mathrm{O}_{6} \mathrm{Na}: 255.0839\left([\mathrm{M}+\mathrm{Na}]^{+}\right)$. Found: 255.0839. 


\section{Further Investigations of Substrate Scope}

\section{6-1. Substrate Scope for Amine Derivatives}

The use of several nitrogen-containing compounds as the substrate was found to provide the corresponding products in good yields without HAT catalyst. Several related reactions have been reported by Nicewicz et al. ${ }^{17}$ At this point, $N$-Boc-protected primary amine successfully afforded the corresponding $\alpha$-alkylated product (14) in the presence of $\mathbf{1 h}$, whereas the same product was not obtained at all in the absence of $\mathbf{1 h}$.

Table S3. C-H Alkylation with amine derivatives.<smiles></smiles>

(3.0 equiv)<smiles>N#CC(C#N)=Cc1ccccc1</smiles>

2<smiles>[R]C(C(c1ccccc1)C(C)N)C([R])N([R])[Po]</smiles>

Acr-Mes ${ }^{+}$(5 mol\%)

1h (0 or $10 \mathrm{~mol} \%)$

$\operatorname{MeCN}(0.1 \mathrm{M}), 25^{\circ} \mathrm{C}$

blue LED, $11 \mathrm{~h}$<smiles>CC(C)(C)OC(=O)N1CCCC1C(c1ccccc1)C(C#N)C#N</smiles>

w/ 1h: $99 \%$ w/o 1h: $99 \%$<smiles>CCC(NC(=O)OCc1ccccc1)C(c1ccccc1)C(C#N)C#N</smiles>

14 w/ 1h: $99 \%$ w/o 1h: $0 \%$<smiles>N#CC(C#N)C(c1ccccc1)C1CCCN1C(=O)c1ccccc1</smiles>

w/ 1h: $94 \%$ w/o 1h: $91 \%$<smiles>N#CC(C#N)C(c1ccccc1)C(Cc1ccccc1)NC(=O)c1ccccc1</smiles>

w/ 1h: N.D. w/o 1h: N.D.<smiles>N#CC(C#N)C(c1ccccc1)C1CCCN1C1CCCCC1</smiles>

w/ 1h: $97 \%$ w/o 1h: $95 \%$<smiles>N#CC(C#N)C(c1ccccc1)C1CCCCN1C(F)(F)F</smiles>

w/ 1h: $<10 \%$ 


\section{6-2. Other Scope of C-H Substrates and Radical Acceptors}

Table S4. C-H Substrates that are not shown in the manuscript.

$$
\begin{aligned}
& \operatorname{Acr}^{+}(5 \mathrm{~mol} \%)
\end{aligned}
$$

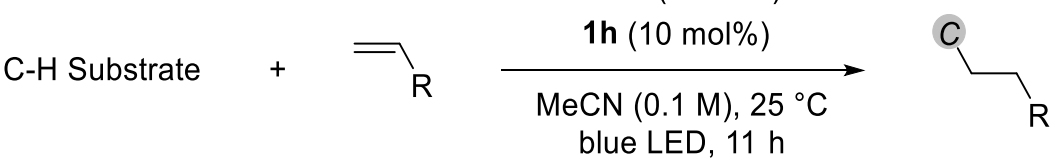

\section{C-H Substrate ${ }^{a}$}<smiles>OCC1CC1</smiles>

(3 equiv)

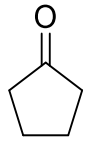

(3 equiv)<smiles>O=C1NC(=O)C2CCCCC12</smiles>

(3 equiv)<smiles>C[C@H](O)[C@@H](C)O</smiles>

(3 equiv)<smiles>N#CC(C#N)=Cc1ccccc1</smiles><smiles>C/C=C(/SOS(=O)(=O)c1ccccc1)S(=O)(=O)c1ccccc1</smiles><smiles>C/C=C(/SOc1ccccc1)S(=O)(=O)Oc1ccccc1</smiles><smiles>C=CC(=O)OC(C(F)(F)F)C(F)(F)F</smiles><smiles>N#CC(C#N)C(c1ccccc1)C(O)C1CC1</smiles>

$74 \%$, d.r. $=1.0: 1$

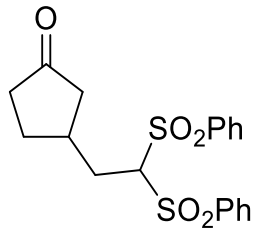

$<10 \%$<smiles>O=C1NC(=O)C2CC(CC(S(=O)(=O)Oc3ccccc3)S(=O)(=O)c3ccccc3)CCC12</smiles>

$32 \%$<smiles></smiles>

$85 \%$, d.r. $=1.5: 1$ (after acidic work up)

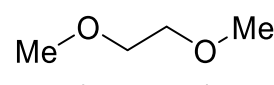

(20 equiv)<smiles>CC=CC(=O)OC(C(F)(F)F)C(F)(F)F</smiles>

$$
\mathrm{Ph}^{-\mathrm{Me}}
$$

(3 equiv)<smiles>CC1CCCO1</smiles>

(20 equiv)<smiles>CCOCC1(C(COCC)(OCC)OCC)CCCCC1</smiles>

(3 equiv)<smiles>CC=CC(=O)OC(C(F)(F)F)C(F)(F)F</smiles><smiles>CC=CC(=O)OC(C(F)(F)F)C(F)(F)F</smiles><smiles>C/C=C(/SOc1ccccc1)S(=O)(=O)Oc1ccccc1</smiles><smiles>COCCOCCCC(=O)OCc1ccccc1</smiles>

$89 \%$, r.r. $=1.2: 1$ (after transesterification)<smiles>COC(CCC(=O)Oc1ccccc1)c1ccccc1</smiles>

$46 \%$, r.r. $=4.0: 1$ (after transesterification)

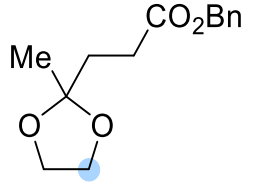

ca. $70 \%$, r.r. $=2.0:$ (after transesterification)

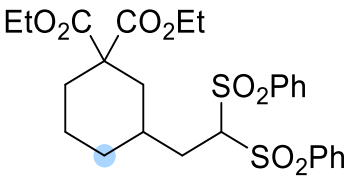

$56 \%$ (isolated), r.r. $=2.5: 1$

\footnotetext{
${ }^{a}$ Unless otherwise noted, NMR yields of the corresponding product are shown.
} 
Table S5. Radical Acceptors that are not shown in the manuscript.

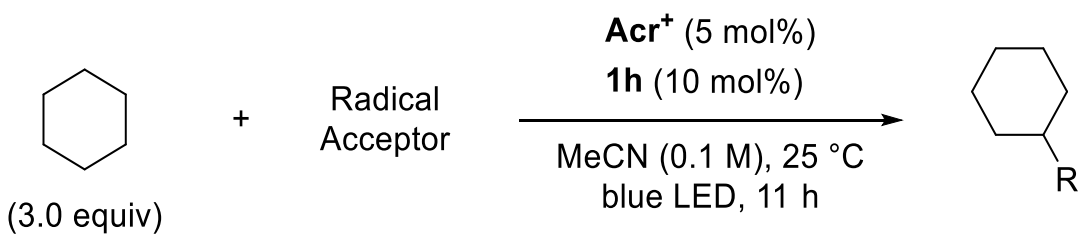

Radical Acceptor ${ }^{2}$

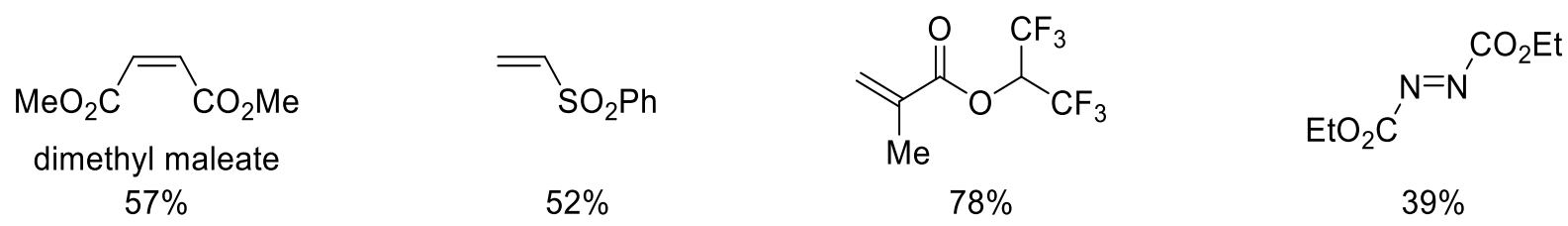

$=\overbrace{\mathrm{CO}_{2} \mathrm{Et}}^{\mathrm{SO}_{2} \mathrm{Ph}}$

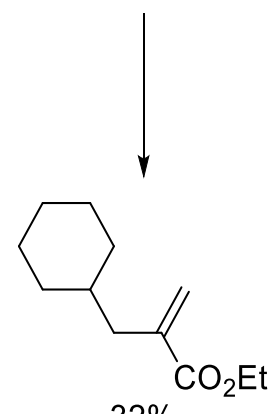

$32 \%$
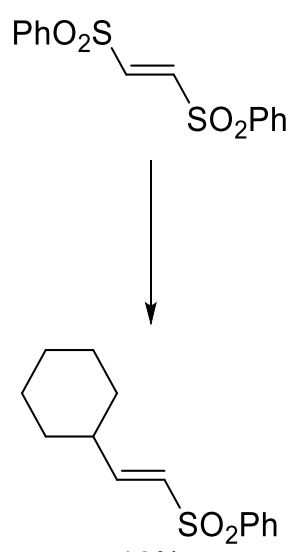

$16 \%$<smiles>O=C1C=CC(=O)N1c1ccccc1</smiles><smiles>CCO/C=C(\C#N)C(C)=O</smiles><smiles>Cc1ccc(S(N)(=O)=O)cc1</smiles>

low yield $(<20 \%)$ or complex mixture

${ }^{a}$ Unless otherwise noted, NMR yields of the corresponding product are shown. 


\section{Stern-Volmer Quenching Studies}

The emission spectra were recorded using a Shimadzu RF-6000 spectrofluorophotometer. All sample solutions were prepared using dry $\mathrm{MeCN}$ and measured immediately after bubbling with argon gas. In a typical experiment, a solution of 3,6-di-tert-butyl-9-mesityl-phenylacridin-10-ium tetrafluoroborate (Mes-Acr ${ }^{+}, 0.20$ $\mathrm{mM}$ ) in dry $\mathrm{MeCN}$ was added to an appropriate amount of quencher in $5 \mathrm{~mL}$ volumetric flask. The solution was transferred to a $1 \mathrm{~cm}^{2}$ quartz quvette. The sample solution was excited at $450 \mathrm{~nm}$ and the luminescence was measured at $510 \mathrm{~nm}$.

The Stern-Volmer quenching studies demonstrated that catalyst $\mathbf{1 h}$ quenched the emission of the excited

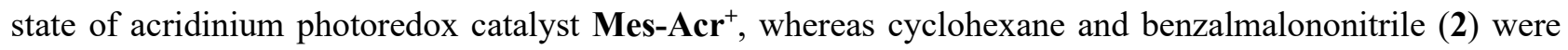
unable to quench the emission.

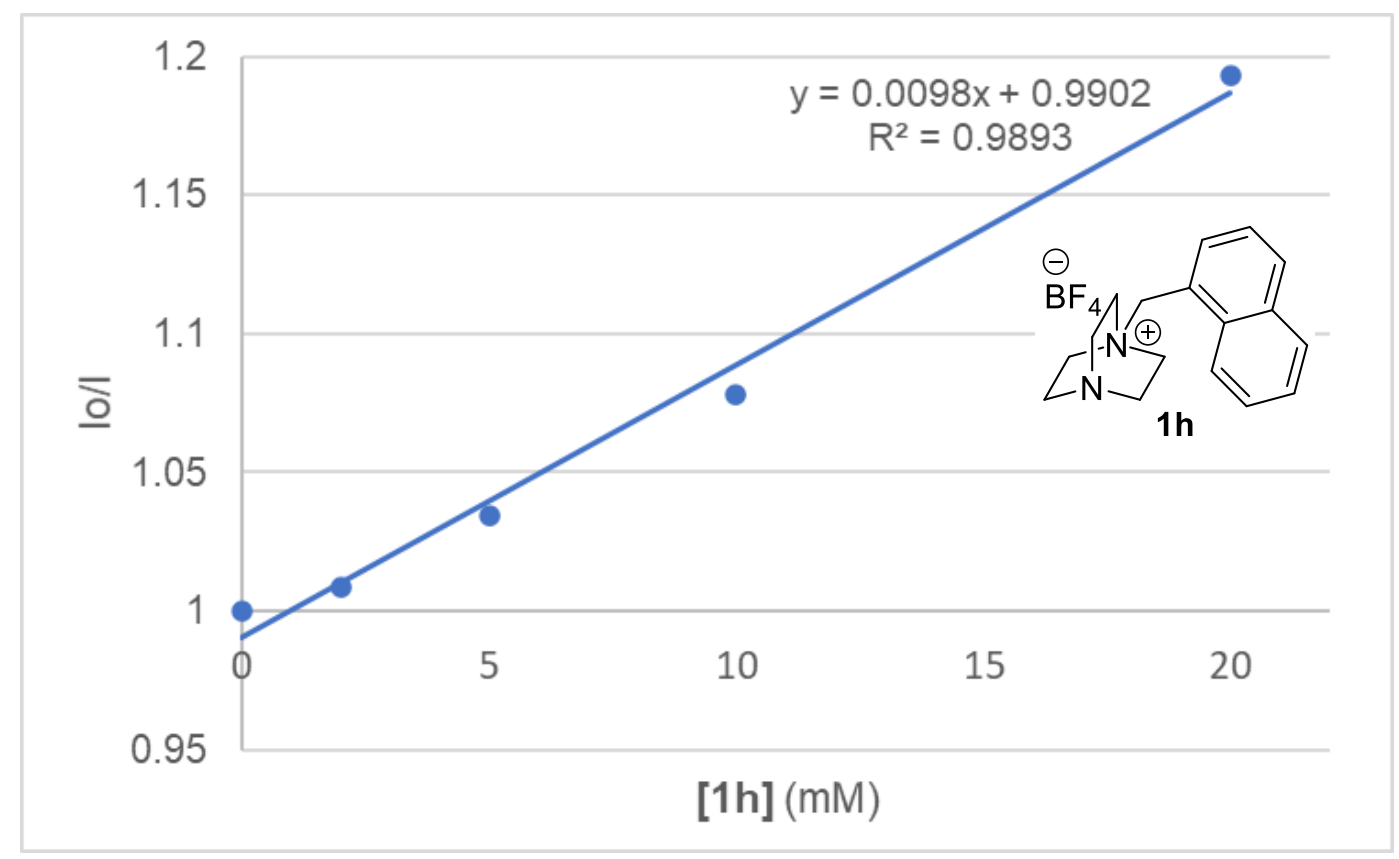

Figure S2. Stern-Volmer Plot for the quenching study using $\mathbf{1 h}$ as a quencher. 


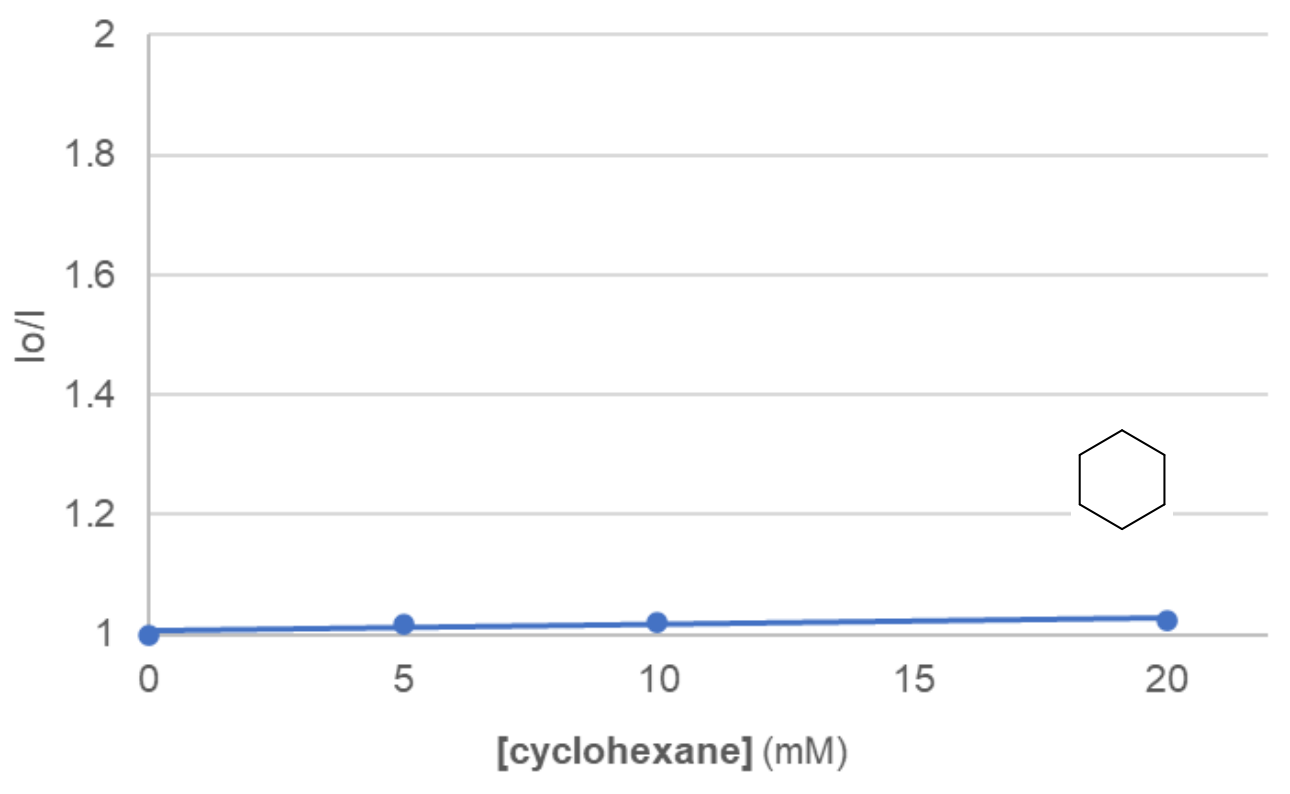

Figure S3. Stern-Volmer Plot for the quenching study using cyclohexane as a quencher.

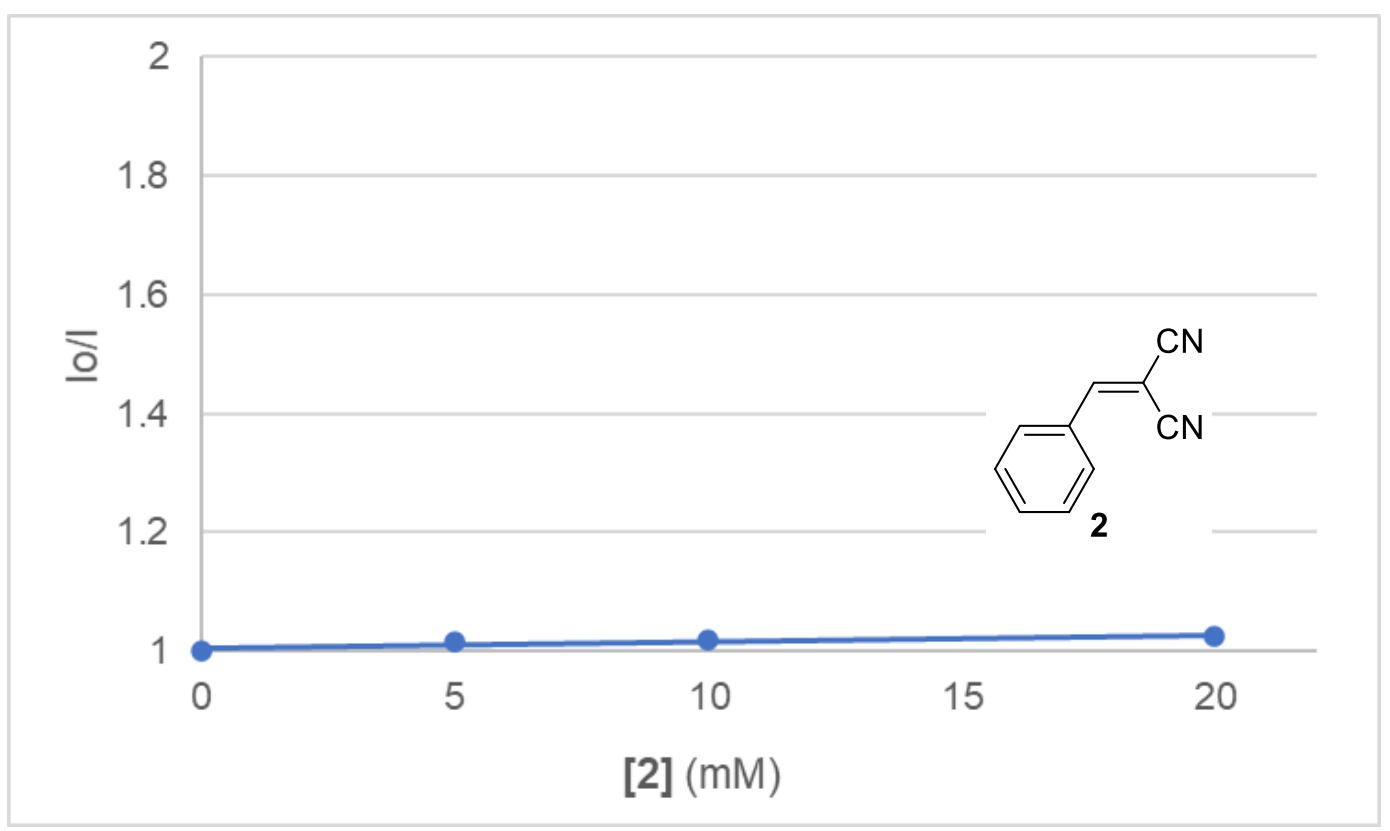

Figure S4. Stern-Volmer Plot for the quenching study using $\mathbf{2}$ as a quencher. 


\section{Cyclic Voltammetry Measurements}

The Cyclic voltammetry (CV) was recorded on ALS model 614E (BAS Inc.) using glassy carbon working electrode, platinum wire counter electrode, and $\mathrm{Ag} / \mathrm{AgNO}_{3}$ reference electrode. Voltammograms were taken at room temperature in a $0.10 \mathrm{M} \mathrm{MeCN}$ solution of tetrabutylammonium tetrafluoroborate $\left(\mathrm{TBABF}_{4}\right)$ containing $5.0 \mathrm{mM}$ (naphthalene and $\mathbf{1 h}$ ) or $90 \mathrm{mM}(\mathbf{1 j})$ of the analyte with the scan rate set to $100 \mathrm{mV} / \mathrm{s}$. For the $\mathrm{CV}$ measurement of naphthalene, ferrocene $(\mathrm{Fc})$ was added as an internal standard. The obtained value was referenced to $\mathrm{Ag} / \mathrm{Ag}^{+}$and converted to $\mathrm{SCE}$ according to the literature. ${ }^{18}$

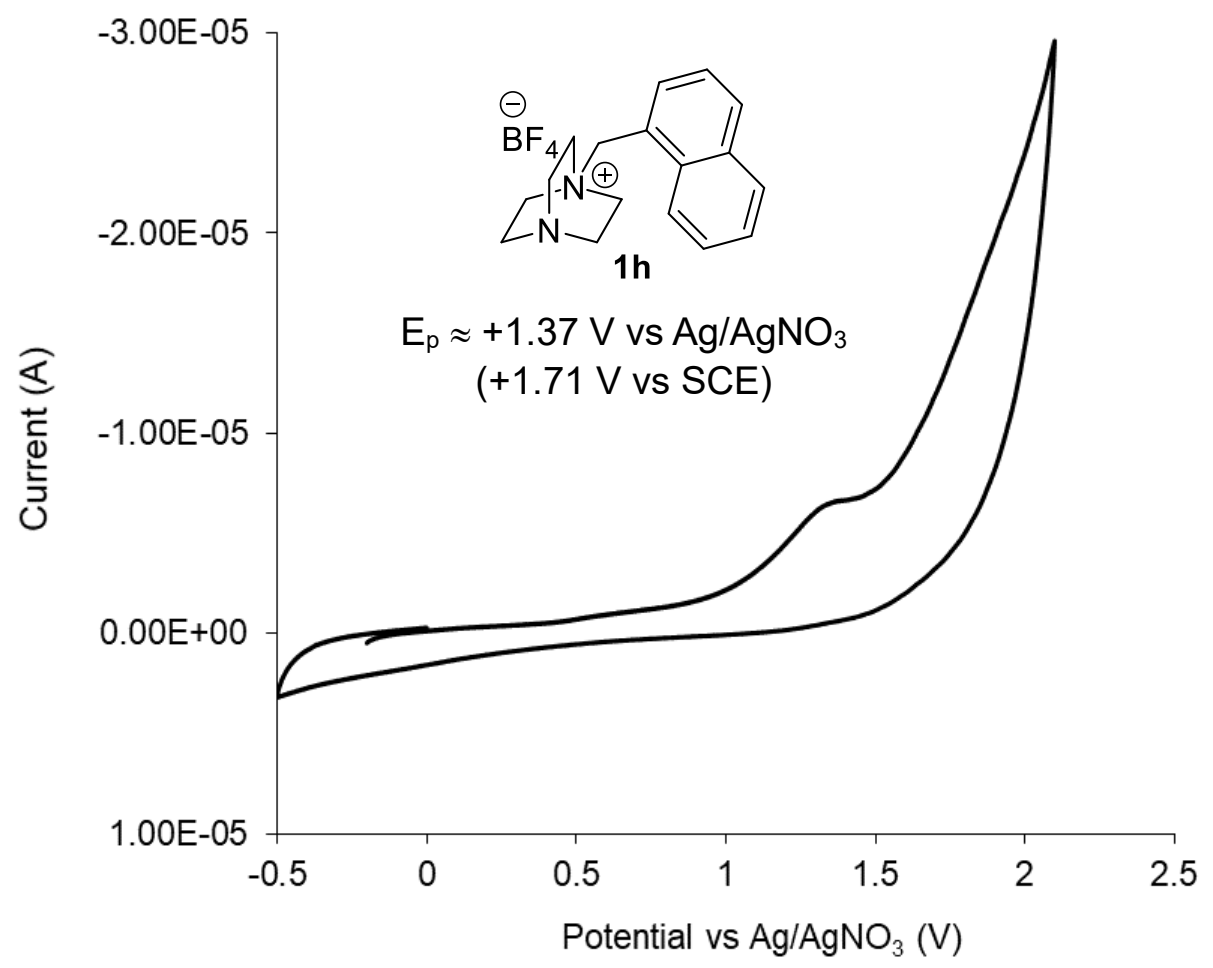

Figure S5. Cyclic voltammogram of $\mathbf{1 h}$. 


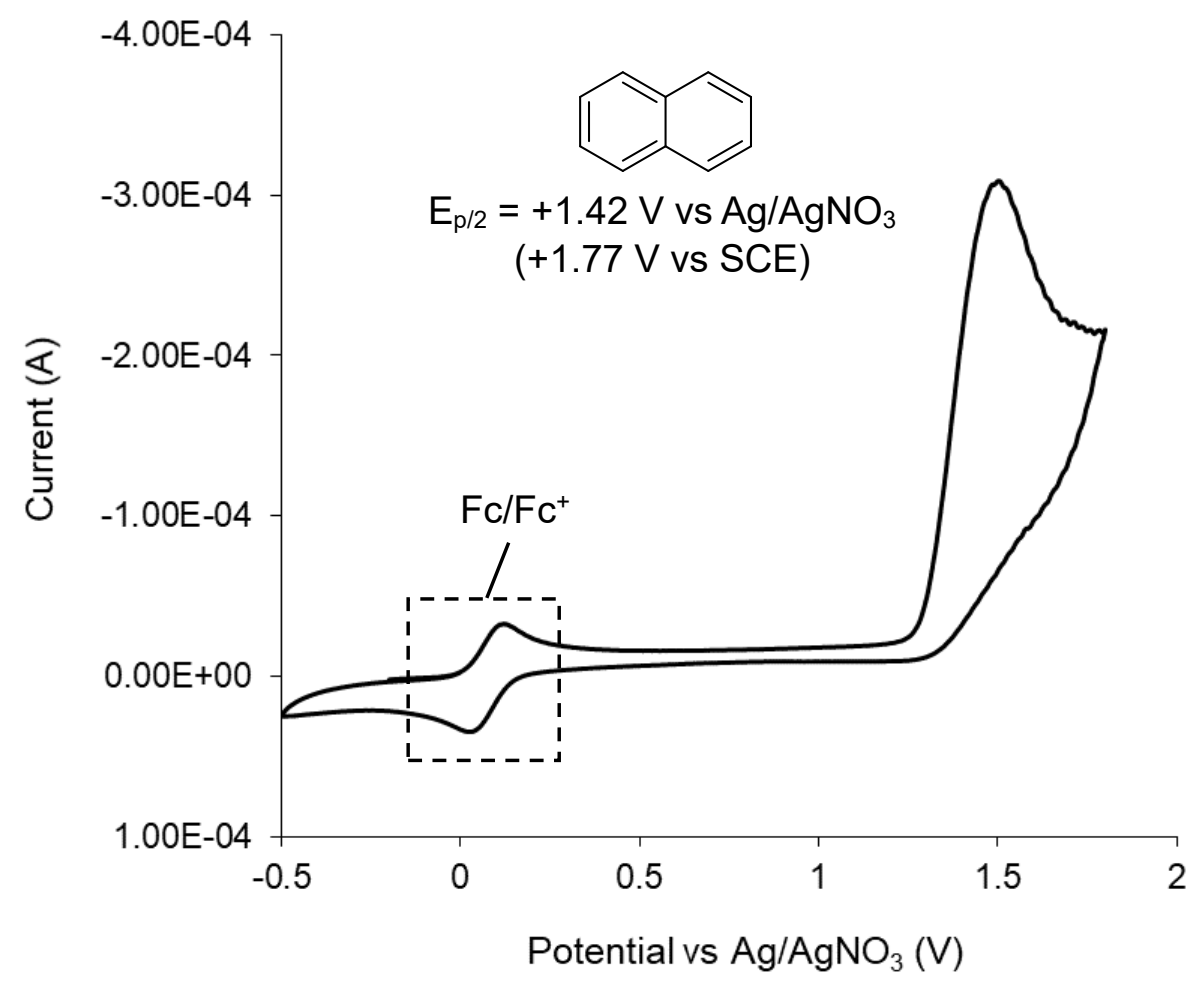

Figure S6. Cyclic voltammogram of naphthalene with ferrocene $(\mathrm{Fc})$ as an internal standard.

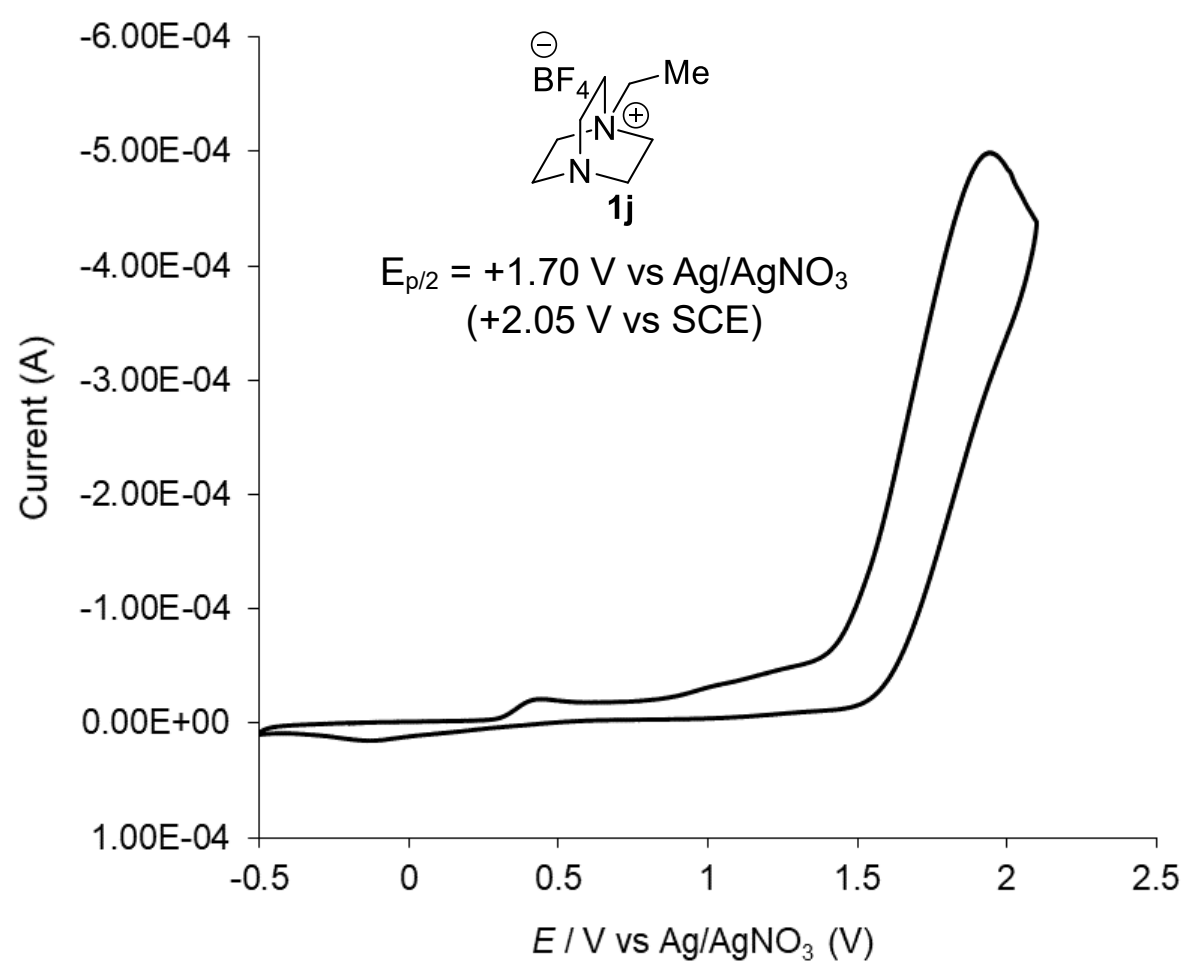

Figure S7. Cyclic voltammogram of $\mathbf{1 j}$. 


\section{Light On/Off Experiment}

The reaction of cyclohexane (3.0 equiv) with benzalmalononitrile (2) was carried out according to General Procedure 1 under alternating periods of irradiation and darkness. For each period of light on/off switching, the yield of the corresponding product 3 was determined by crude ${ }^{1} \mathrm{H}$ NMR using 1,1,2,2-tetrachloroethane as an internal standard. These results showed a total interruption of the reaction progress in the absence of light and recuperation of reactivity on further illumination, indicating that light is a necessary component of the reaction. Even though we could not definitively rule out a radical-chain process, the data implies that any chain-propagation process must be short-lived.

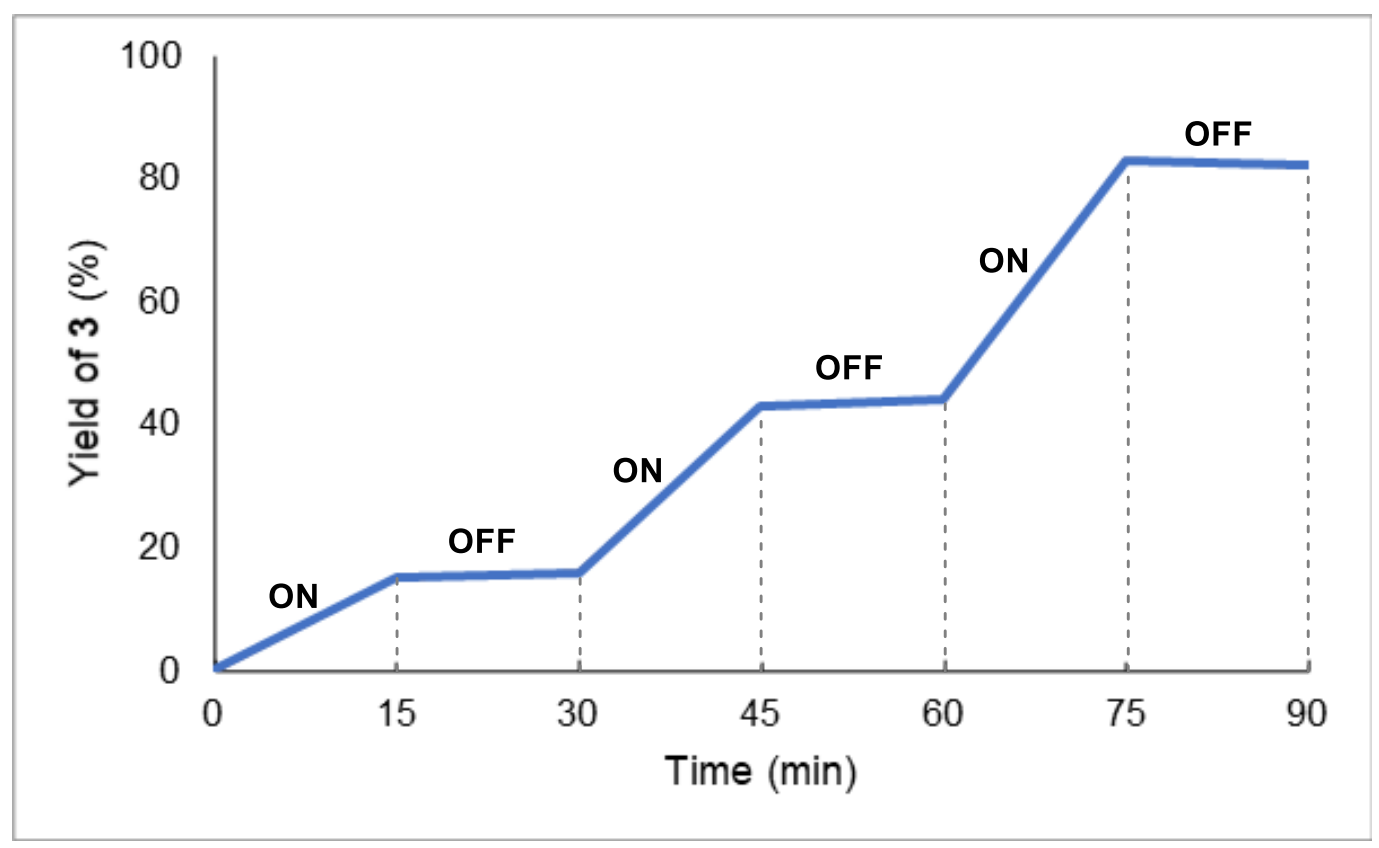

Figure S8. Time profile of product yield for light on/off experiment. 


\section{Mechanistic Studies}

\section{0-1. Deuterium Labeling Studies}

- Intermolecular competitive reaction<smiles>C1CCCCC1</smiles>

(1.5 equiv)<smiles>c1ccccc1</smiles>

(1.5 equiv)

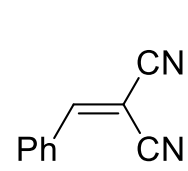

2

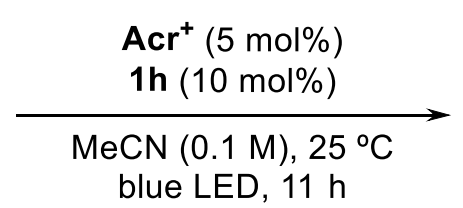

$K I E=2.6$

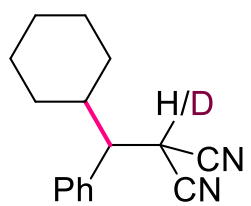

3/3-d, $69 \%$

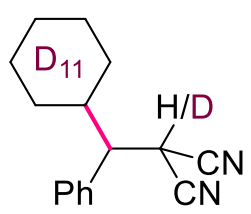

3'/3'-d, $27 \%$

- Parallel reaction<smiles>C1CCCCC1</smiles>

(3.0 equiv)

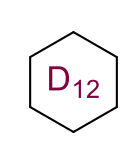

(3.0 equiv)<smiles>N#CC(C#N)=Cc1ccccc1</smiles>

2<smiles>N#CC(C#N)=Cc1ccccc1</smiles>

2
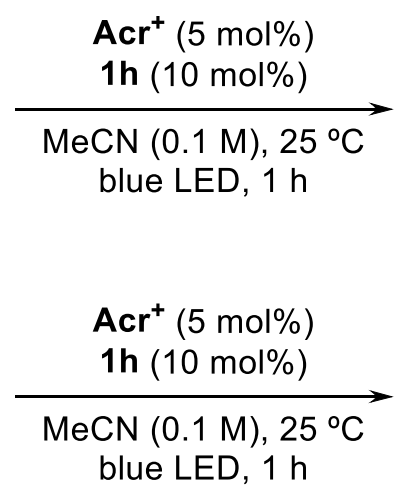

$K I E=1.4$

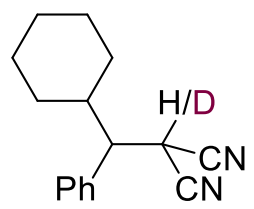

3/3-d, $70 \%$

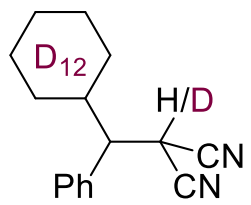

3' $^{\prime} / 3^{\prime}-$ d, $49 \%$

Scheme S1. Deuterium labeling experiments.

Several reactions were carried out according to General Procedure 1 using cyclohexane and cyclohexane$\mathrm{d} 12$ as the $\mathrm{C}-\mathrm{H}(\mathrm{D})$ substrates. When the reaction was conducted using 1:1 mixture of cyclohexane (1.5 equiv) and cyclohexane-d12 (1.5 equiv), the KIE was found to be 2.6. Next, the reactions were conducted using cyclohexane (3.0 equiv) and cyclohexane-d12 (3.0 equiv) separately. These results indicated the KIE of parallel reaction to be 1.4. These values are in agreement with the mechanism of hydrogen-atom abstraction from unactivated $\mathrm{C}-\mathrm{H}$ bond by dicationic aminium radical. ${ }^{19}$ 


\section{0-2. Radical Inhibiting Experiment}

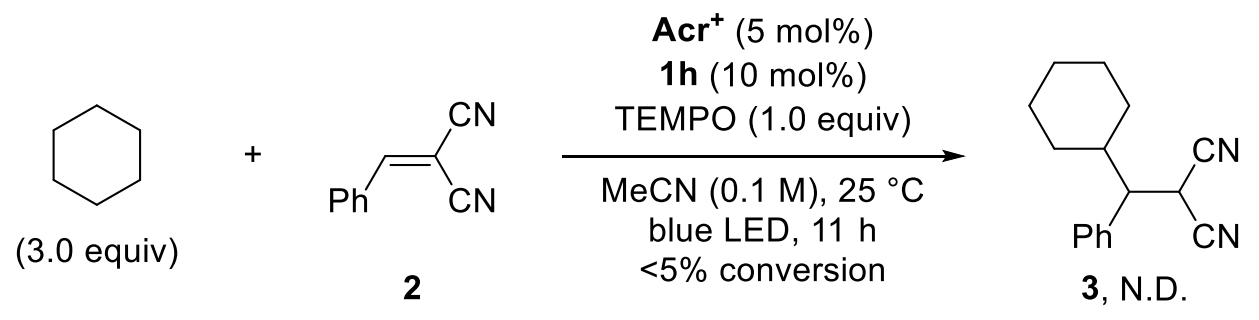

Scheme S2. Reaction with the addition of TEMPO.

The reaction of cyclohexane with benzalmalononitrile (2) was carried out according to General Procedure 1 with the addition of 2,2,6,6-Tetramethylpiperidine 1-Oxyl (TEMPO, $31.3 \mathrm{mg}, 0.20 \mathrm{mmol}, 1.0$ equiv) as a radical scavenger. After the reaction, the crude mixture was analyzed by ${ }^{1} \mathrm{H}$ NMR and HRMS, which indicated that the reaction was totally inhibited and product 3 was not detected at all. 


\section{Computational Methods}

\section{1-1. Suitable basis sets for geometry optimization.}

Geometry optimization was performed using the (U)M06-2X functional, ${ }^{20}$ because it shows good performance for evaluating bond dissociation energy (BDE) of $\mathrm{N}^{+}-\mathrm{H}$ bonds in the conjugate acids of amines. ${ }^{21}$ In order to search suitable basis sets for geometry optimization, we employed a variety of basis sets to optimize the geometry of $\mathrm{Me}_{2} \mathrm{NH}$, quinuclidine, and $\mathrm{DABCO}$ and compared their BDEs for $\mathrm{N}^{+}-\mathrm{H}$ bonds with the experimental values. ${ }^{22}$ As shown in Table S6, all basis sets provide similar values of BDEs for $\mathrm{N}^{+}-\mathrm{H}$ bonds. Considering computational costs, we employed $6-311 \mathrm{G}(\mathrm{d}, \mathrm{p})$ for geometry optimization. The solvent effect of acetonitrile was considered by employing the SMD model. ${ }^{23}$

Table S6. Homolytic bond dissociation energies (BDEs) for the $\mathrm{N}^{+}-\mathrm{H}$ bonds in the conjugate acids of amines estimated in acetonitrile using M06-2X.

\begin{tabular}{ccccccc}
\hline & (1) $\mathbf{M e}_{2} \mathbf{N H}$ & (2) quinuclidine & (3) DABCO & $\Delta(\mathbf{1 - 2})$ & $\Delta(\mathbf{1 - 3})$ & $\Delta(\mathbf{2 - 3})$ \\
\hline Ref $^{22}$ & 101.5 & 100.0 & 91.3 & 1.5 & 10.2 & 8.7 \\
$\mathbf{6 - 3 1 1 + G ( 2 d f , p )}$ & 110.3 & 108.0 & 94.2 & 2.3 & 16.1 & 13.8 \\
$\mathbf{6 - 3 1 + G ( 2 d f , p )}$ & 111.4 & 108.6 & 94.9 & 2.8 & 16.5 & 13.7 \\
$\mathbf{6 - 3 1 1 G ( 2 d , p )}$ & 110.9 & 108.4 & 94.8 & 2.5 & 16.1 & 13.6 \\
$\mathbf{6 - 3 1 1 G ( d , p )}$ & 111.0 & 108.8 & 95.1 & 2.2 & 15.9 & 13.7 \\
\hline
\end{tabular}

\section{1-2. Ionization potential (IP)}

In order to evaluate the ionization potential for nitrogen radical cation (N_IP) and aryl radical cation ( $\pi$ IP), two model reactions were proposed, as shown in Scheme S3. All geometries were optimized by M06-2X functional using 6-311G(d,p) basis sets. Then, the single point calculation was performed using $\operatorname{CCSD}(\mathrm{T}),{ }^{24}$ $\mathrm{CCSD},{ }^{24} \mathrm{MP} 4(\mathrm{SDQ}),{ }^{25} \mathrm{MP} 4(\mathrm{DQ}),{ }^{25} \mathrm{MP} 4(\mathrm{D}),{ }^{26} \mathrm{MP} 3,{ }^{26} \mathrm{MP} 2,{ }^{27}$ and M06-2X, where cc-PVTZ basis sets ${ }^{28}$ were used without polarizations. The solvent effects of acetonitrile were considered by employing the SMD model for both geometry optimization and single-point calculations. As shown in Table S7, the IP value for producing nitrogen radical cation is smaller than that for producing phenyl radical cation in all cases. It should be noted that M06-2X reproduced well the IP values calculated by the $\operatorname{CCSD}(\mathrm{T})$ method, indicating that the M06-2X can be applied well to the calculations of these two IP values. In summary, we employed M06-2X with 6$311 \mathrm{G}(\mathrm{d}, \mathrm{p})$ basis sets for IP evaluation.

All these calculations were carried out using Gaussian 16 program. ${ }^{29}$ 
(a)<smiles>CC(N(C)C)N(C)C</smiles>

(b)

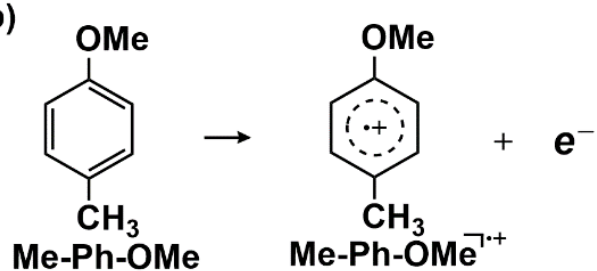

Scheme S3. Model reactions to evaluate the ionization potential.

Table S7. Ionization potential (IP, $\mathrm{kcal} / \mathrm{mol}$ ) of model reaction shown in Scheme S3 with various methods.

\begin{tabular}{cccc}
\hline IP (kcal/mol) & $\begin{array}{c}\text { (1) } \mathbf{N M e}_{3} \\
\text { IP from N }\end{array}$ & $\begin{array}{c}\text { (2) Me-Ph-OMe } \\
\text { IP from Ph }\end{array}$ & $\Delta(\mathbf{2 - 1})$ \\
\hline MP2 & 121.68 & 145.23 & 23.55 \\
MP3 & 116.72 & 135.87 & 19.15 \\
MP4(D) & 117.55 & 138.02 & 20.47 \\
MP4(DQ) & 116.65 & 134.90 & 18.25 \\
MP4(SDQ) & 116.32 & 132.16 & 15.84 \\
CCSD & 115.29 & 129.34 & 14.05 \\
CCSD(T) & 117.09 & 131.34 & 14.25 \\
M06-2X & 120.52 & 134.03 & 13.51 \\
\hline
\end{tabular}




\section{1-3. Ionization Potential (IP) and Bond Dissociation Energies (BDEs) of $\mathbf{N}^{+}-\mathrm{H}$ bonds for HAT catalysts 1}

(a) IP

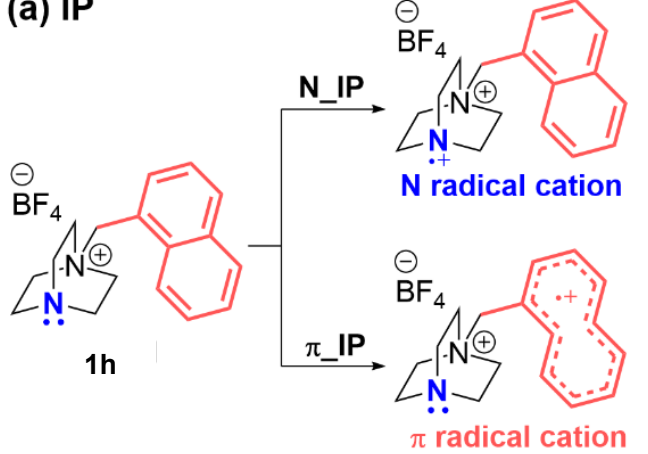

(b) BDE

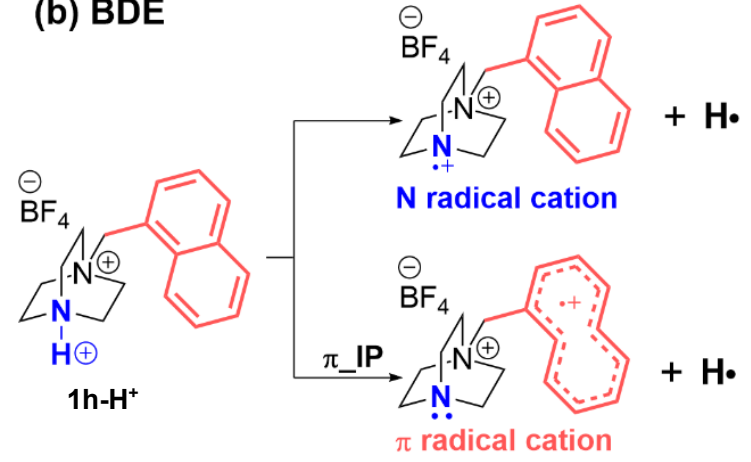

Scheme S4. Assumed reactions to evaluate the IP and BDE for $\mathbf{1 h}$.

Considering nitrogen lone pair orbital and aryl $\pi$-orbital could participate in ionization, we calculated ionization potential for these two moieties, as shown in Scheme S4(a) where 1h is presented as one example; the ionization from the $\mathrm{N}$ lone-pair orbital is name $\mathrm{N} \_I P$ and that from the p-orbital is $\pi$ IP, hereinafter. Geometry optimizations and vibrational frequency calculations were persformed at the (U)M06-2X/6$311 \mathrm{G}(\mathrm{d}, \mathrm{p})$ level, where solvation effect of acetonitrile was considered using the SMD model. We also carried out similar calculation for $\mathbf{1 a}-\mathbf{g}$, as listed in Table S8. Apparently, the N_IP value is slightly smaller than $\pi$ IP value for $\mathbf{1 h}$. However, the experimental results by $\mathrm{CV}$ show that the half-wave potential is respectively 2.05 $\mathrm{V}$ and $1.77 \mathrm{~V}$ (vs SCE) for the one-electron oxidation of (the amine moiety of) $\mathbf{1} \mathbf{j}$ and that of naphthalene. These results indicate that the N_IP is likely to be underestimated about $6.5 \mathrm{kcal} / \mathrm{mol}$. Thus, it is likely to increase the N_IP value by $6.5 \mathrm{kcal} / \mathrm{mol}$. Because the SMD model was used in calculations of Table S7, more complicated factor would exist; One of such factors is the position of counter anion, because its position is likely to influence the one-electron oxidation. Because the optimization of the position of counter cation is very difficult, we employed here the empirical correction for the N_IP value.

Accordingly, aryl radical cation is more stable than nitrogen radical cation for $\mathbf{1 c}, \mathbf{1 d}, \mathbf{1 g}$, and $\mathbf{1 h}$, as shown in Table S8, whereas nitrogen radical cation is more stable than aryl radical cation for the others. Based on these results, BDEs of the $\mathrm{N}^{+}-\mathrm{H}$ bonds in the conjugate acids of HAT catalysts $\mathbf{1}$ were evaluated, where the most stable radical cation was employed. The blue color in Table S8 means the most stable IP in the molecule. 
Table S8. Yields, IP values and BDEs for HAT catalysts $\mathbf{1 .}$

\begin{tabular}{|c|c|c|c|c|c|c|c|c|}
\hline & $1 \mathbf{a}$ & $1 b$ & 1c & 1d & $1 e$ & 1f & $1 \mathrm{~g}$ & $1 \mathrm{~h}$ \\
\hline (Exp.) yield & $14 \%$ & $34 \%$ & $93 \%$ & $9 \%$ & $35 \%$ & $24 \%$ & $89 \%$ & $95 \%$ \\
\hline (Cal.) yield & $23 \%$ & $24 \%$ & $87 \%$ & $16 \%$ & $25 \%$ & $23 \%$ & $102 \%$ & $78 \%$ \\
\hline N_IP & 146.1 & 143.4 & 143.3 & 143.4 & 143.7 & 144.4 & 143.5 & 143.9 \\
\hline N_IP + 6.5 & 152.6 & 149.9 & 149.8 & 149.9 & 150.2 & 150.9 & 150.0 & 150.4 \\
\hline$\pi$ IP & - & 161.8 & 144.8 & 137.9 & 168.9 & - & 147.6 & 144.7 \\
\hline BDE & $\begin{array}{c}106.1 \\
\left(\mathrm{~N} \_I P\right)\end{array}$ & $\begin{array}{c}104.9 \\
\left(N_{-} I P\right)\end{array}$ & $\begin{array}{l}106.6 \\
\left(\pi \_ \text {IP }\right)\end{array}$ & $\begin{array}{c}99.7 \\
\left(\pi \_ \text {IP }\right)\end{array}$ & $\begin{array}{c}105.1 \\
\left(\mathrm{~N} \_I P\right)\end{array}$ & $\begin{array}{c}105.3 \\
\left(\mathrm{~N} \_I P\right)\end{array}$ & $\begin{array}{l}108.9 \\
\left(\pi \_I P\right)\end{array}$ & $\begin{array}{l}106.0 \\
\left(\pi \_ \text {IP }\right)\end{array}$ \\
\hline$\varepsilon($ HOMO) & $\begin{array}{c}-8.23 \\
\left(\mathrm{~N} \_I P\right)\end{array}$ & $\begin{array}{c}-8.10 \\
\left(\mathrm{~N} \_I P\right)\end{array}$ & $\begin{array}{l}-7.73 \\
\left(\pi \_I P\right)\end{array}$ & $\begin{array}{c}-7.41 \\
\left(\mathrm{~N} \_I P\right)\end{array}$ & $\begin{array}{c}-8.12 \\
\left(\mathrm{~N} \_I P\right)\end{array}$ & $\begin{array}{c}-8.14 \\
\left(\mathrm{~N} \_I P\right)\end{array}$ & $\begin{array}{l}-7.78 \\
\left(\pi \_ \text {IP }\right)\end{array}$ & $\begin{array}{l}-7.53 \\
\left(\pi \_ \text {IP }\right)\end{array}$ \\
\hline$\varepsilon($ HOMO-1) & $\begin{array}{l}-10.57 \\
\left(\pi \_ \text {IP) }\right.\end{array}$ & $\begin{array}{l}-8.61 \\
\left(\pi \_ \text {IP }\right)\end{array}$ & $\begin{array}{c}-8.10 \\
\left(\mathrm{~N} \_I P\right)\end{array}$ & $\begin{array}{c}-8.10 \\
\left(\mathrm{~N} \_I P\right)\end{array}$ & $\begin{array}{l}-8.94 \\
\left(\pi \_I P\right)\end{array}$ & $\begin{array}{l}-9.30 \\
\left(\pi \_ \text {IP }\right)\end{array}$ & $\begin{array}{c}-8.11 \\
\left(\mathrm{~N} \_I P\right)\end{array}$ & $\begin{array}{c}-8.13 \\
\left(\mathrm{~N} \_I P\right)\end{array}$ \\
\hline
\end{tabular}

The yield is related to IP and BDE; Actually, the yield is represented well as a function of IP and BDE, as shown by eq $\mathrm{S} 1$.

$$
\text { yield }(\%)=-7.15[\mathrm{IP}]+15.47[\mathrm{BDE}]-527.2 \quad(\mathrm{R}=0.94) \quad \text { eq }(\mathrm{S} 1)
$$

where the $\mathrm{R}$ value for linear relation is 0.94 . This relation indicates that (i) the IP and BDE are determination factors for activity of these catalysts, (ii) the small IP is better but the large BDE is better, and (iii) the BDE plays a more important role than IP for this reaction. 


\section{References}

[1] Chauhan, M. S.; Yadav, G. D.; Hussain, F.; Singh, S. N-Fluorobenzenaminium Tetrafluoroborate Generated in Situ by Aniline and Selectfluor as a Reusable Catalyst for the Ring Opening of Epoxides with Amines under Microwave Irradiation. Catal. Sci. Technol. 2014, 4, 3945-3952.

[2] Pine, S. H.; Cheney, J.; Catto, B.; Petersen, J. D. Base-Promoted Reactions of Bicyclic Mono- and Diquaternary Ammonium Salts. J. Org. Chem. 1974, 39, 130-133.

[3] Pfeifer, L.; Gouverneur, V. Controlled Single and Double Iodofluorination of Alkynes with DIH- and HFBased Reagents. Org. Lett. 2018, 20, 1576-1579.

[4] Li, H.; Yang, M.; Zhang, X.; Yan, L.; Li, J.; Qi, Y. Mesoporous Silica-Supported Copper-Catalysts for Homocoupling Reaction of Terminal Alkynes at Room-Temperature. New J. Chem. 2013, 37, 1343-1349.

[5] Schneider, C.; Jackstell, R.; Maes, B. U. W.; Beller, M. Palladium-Catalyzed Alkoxycarbonylation of secBenzylic Ethers. Eur. J. Org. Chem. 2020, 8, 932-936.

[6] Bantu, R.; Mereyala, H. B.; Nagarapu, L.; Kantevari, S. 3-O-Acyl Triggered Tandem Lewis Acid Catalyzed Intramolecular Cyclization of Diacetone Glucose Derivatives to 5-O-Acyl-3,6-Anhydro-D-Glucose. Tetrahedron Lett. 2011, 52, 4854-4856.

[7] Mukherjee, S.; Maji, B.; Tlahuext-Aca, A.; Glorius, F. Visible-Light-Promoted Activation of Unactivated $\mathrm{C}\left(\mathrm{sp}^{3}\right)-\mathrm{H}$ Bonds and Their Selective Trifluoromethylthiolation. J. Am. Chem. Soc. 2016, 138, 1620016203.

[8] Fan, X.-Z.; Rong, J.-W.; Wu, H.-L.; Zhou, Q.; Deng, H.-P.; Tan, J. D.; Xue, C.-W.; Wu, L.-Z.; Tao, H.-R.; $\mathrm{Wu}, \mathrm{J}$. Eosin Y as a Direct Hydrogen-Atom Transfer Photocatalyst for the Functionalization of C-H Bonds. Angew. Chem., Int. Ed. 2018, 57, 8514-8518.

[9] Deng, H.-P.; Zhou, Q.; Wu, J. Microtubing-Reactor-Assisted Aliphatic C-H Functionalization with $\mathrm{HCl}$ as a Hydrogen-Atom-Transfer Catalyst Precursor in Conjunction with an Organic Photoredox Catalyst. Angew. Chem., Int. Ed. 2018, 57, 12661-12665.

[10] Jia, P.; Li, Q.; Poh, W. C.; Jiang, H.; Liu, H.; Deng, H.; Wu, J. Light-Promoted Bromine-Radical-Mediated Selective Alkylation and Amination of Unactivated C $\left(\mathrm{sp}^{3}\right)-\mathrm{H}$ Bonds. Chem 2020, 6, 1766-1776.

[11] Liu, R.; Chia, S. P. M.; Goh, Y. Y.; Cheo, H. W.; Fan, B.; Li, R.; Zhou, R.; Wu, J. Visible-Light-Mediated Regioselective Allylation, Benzylation, and Silylation of Methylene-Malononitriles via PhotoredoxInduced Radical Cation Fragmentation. Eur. J. Org. Chem. 2020, 2020, 1459-1465.

[12] Li, L.; Guo, S.; Wang, Q.; Zhu, J. Acyl Radicals from Benzothiazolines: Synthons for Alkylation, Alkenylation, and Alkynylation Reactions. Org. Lett. 2019, 21, 5462-5466.

[13] Rohe, S.; Morris, A. O.; McCallum, T.; Barriault, L. Hydrogen Atom Transfer Reactions via Photoredox Catalyzed Chlorine Atom Generation. Angew. Chem., Int. Ed. 2018, 57, 15664-15669.

[14] Morofuji, T.; Matsui, Y.; Ohno, M.; Ikarashi, G.; Kano, N. Photocatalytic Giese-Type Reaction with Alkylsilicates Bearing C,O-Bidentate Ligands. Chem.-Eur. J. 2021, 27, 6713-6718. 
[15] Kamijo, S.; Takao, G.; Kamijo, K.; Tsuno, T.; Ishiguro, K.; Murafuji, T. Alkylation of Nonacidic C( sp $\left.^{3}\right)-\mathrm{H}$ Bonds by Photoinduced Catalytic Michael-Type Radical Addition. Org. Lett. 2016, 18, 4912-4915.

[16] Ma, Z.-Y.; Li, M.; Guo, L.-N.; Liu, L.; Wang, D.; Duan, X.-H. Sulfonamide as Photoinduced HydrogenAtom Transfer Catalyst for Regioselective Alkylation of $\mathrm{C}\left(\mathrm{sp}^{3}\right)-\mathrm{H}$ Bonds Adjacent to Heteroatoms. Org. Lett. 2021, 23, 474-479.

[17] (a) McManus, J. B.; Onuska, N. P. R.; Nicewicz, D. A. Generation and Alkylation of $\alpha$-Carbamyl Radicals via Organic Photoredox Catalysis. J. Am. Chem. Soc. 2018, 140, 9056-9060. (b) McManus, J. B.; Onuska, N. P. R.; Jeffreys, M. S.; Goodwin, N. C.; Nicewicz, D. A. Site-Selective C-H Alkylation of Piperazine Substrates via Organic Photoredox Catalysis. Org. Lett. 2020, 22, 679-683.

[18] Pavlishchuk, V. V.; Addison, A. W. Conversion Constants for Redox Potentials Measured versus Different Reference Electrodes in Acetonitrile Solutions at $25^{\circ}$ C. Inorganica Chim. Acta 2000, 298, 97-102.

[19] (a) Pitts, C. R.; Bloom, S.; Woltornist, R.; Auvenshine, D. J.; Ryzhkov, L. R.; Siegler, M. A.; Lectka, T. Direct, Catalytic Monofluorination of $\mathrm{sp}^{3} \mathrm{C}-\mathrm{H}$ Bonds: A Radical-Based Mechanism with Ionic Selectivity. J. Am. Chem. Soc. 2014, 136, 9780-9791. (b) Michaudel, Q.; Thevenet, D.; Baran, P. S. Intermolecular Ritter-Type C-H Amination of Unactivated sp ${ }^{3}$ Carbons. J. Am. Chem. Soc. 2012, 134, 2547-2550.

[20] Zhao, Y.; Truhlar, D. G. The M06 Suite of Density Functionals for Main Group Thermochemistry, Thermochemical Kinetics, Noncovalent Interactions, Excited States, and Transition Elements: Two New Functionals and Systematic Testing of Four M06-class Functionals and 12 Other Functionals. Theor. Chem. Acc. 2008, 120, 215-241.

[21] O'reilly, R. J.; Karton, A.; Radom, L. N-H and N-Cl Homolytic Bond Dissociation Energies and Radical Stabilization Energies: An Assessment of Theoretical Procedures through Comparison with Benchmarkquality W2w Data. Int. J. Quantum Chem. 2012, 112, 1862-1878.

[22] Liu, W.-Z.; Bordwell, F. G. Gas-Phase and Solution-Phase Homolytic Bond Dissociation Energies of $\mathrm{H}-\mathrm{N}^{+}$Bonds in the Conjugate Acids of Nitrogen Bases. J. Org. Chem. 1996, 61, 4778-4783.

[23] Marenich, A. V.; Cramer, C. J.; Truhlar, D. G. Universal Solvation Model Based on Solute Electron Density and on a Continuum Model of the Solvent Defined by the Bulk Dielectric Constant and Atomic Surface Tensions. J. Phys. Chem. B, 2009, 113, 6378-6396.

[24] (a) Cížek, J. In Advances in Chemical Physics; Hariharan, P. C. Ed.; Wiley Interscience: New York, 1969; Vol. 14, p 35. (b) Purvis III, G. D.; Bartlett, R. J. A Full Coupled-cluster Singles and Doubles Model: The Inclusion of Disconnected Triples. J. Chem. Phys. 1982, 76, 1910-1918. (c) Scuseria, G. E.; Janssen, C. L.; Schaefer III, H. F. An Efficient Reformulation of the Closed-shell Coupled Cluster Single and Double Excitation (CCSD) Equations. J. Chem. Phys. 1988, 89, 7382-7387. (d) Scuseria, G. E.; Schaefer III, H. F. Is Coupled Cluster Singles and Doubles (CCSD) More Computationally Intensive than Quadratic Configuration Interaction (QCISD)? J. Chem. Phys. 1989, 90, 3700-3703.

[25] Krishnan, R.; Pople, J. A. Approximate fourth-order perturbation theory of the electron correlation energy. Int. J. Quantum Chem. 1978, 14, 91-100. 
[26] (a) Pople, J. A.; Binkley, J. S.; Seeger, R. Theoretical Models Incorporating Electron Correlation. Int. J. Quantum Chem. Suppl. 1976, 10, 1-19. (b) Pople, J. A.; Seeger, R.; Krishnan, R. Variational Configuration Interaction Methods and Comparison with Perturbation Theory. Int. J. Quantum Chem. Suppl. 1977, 12, 149-163.

[27] (a) Head-Gordon, M.; Pople, J. A.; Frisch, M. J. MP2 Energy Evaluation by Direct Methods. Chem. Phys. Lett. 1988, 153, 503-506. (b) Saebø, S.; Almlöf, J. Avoiding the Integral Storage Bottleneck in LCAO Calculations of Electron Correlation. Chem. Phys. Lett. 1989, 154, 83-89. (c) Frisch, M. J.; Head-Gordon, M.; Pople, J. A. A Direct MP2 Gradient Method. Chem. Phys. Lett. 1990, 166, 275-280. (d) Frisch, M. J.; Head-Gordon, M.; Pople, J. A. Semi-direct Algorithms for the MP2 Energy and Gradient. Chem. Phys. Lett. 1990, 166, 281-289. (e) Head-Gordon, M.; Head-Gordon, T. Analytic MP2 Frequencies without Fifth-order Storage. Theory and Application to Bifurcated Hydrogen Bonds in the Water Hexamer. Chem. Phys. Lett. 1994, 220, 122-128.

[28] (a) Krishnan, R.; Binkley, J. S.; Seeger, R.; Pople, J. A. Self-consistent Molecular Orbital Methods. XX. A Basis Set for Correlated Wave Functions. J. Chem. Phys. 1980, 72, 650-654. (b) McLean, A. D.; Chandler, G. S. Contracted Gaussian Basis Sets for Molecular Calculations. I. Second Row Atoms, Z=1118. J. Chem. Phys. 1980, 72, 5639-5648.

[29] Frisch, M. J.; Trucks, G. W.; Schlegel, H. B.; Scuseria, G. E.; Robb, M. A.; Cheeseman, J. R.; Scalmani, G.; Barone, V.; Mennucci, B.; Petersson, G. A.; Nakatsuji, H.; Caricato, M.; Li, X.; Hratchian, H. P.; Izmaylov, A. F.; Bloino, J.; Zheng, G.; Sonnenberg, J. L.; Hada, M.; Ehara, M.; Toyota, K.; Fukuda, R.; Hasegawa, J.; Ishida, M.; Nakajima, T.; Honda, Y.; Kitao, O.; Nakai, H.; Vreven, T.; Montgomery, J. A., Jr.; Peralta, J. E.; Ogliaro, F.; Bearpark, M.; Heyd, J. J.; Brothers, E.; Kudin, K. N.; Staroverov, V. N.; Kobayashi, R.; Normand, J.; Raghavachari, K.; Rendell, A.; Burant, J. C.; Iyengar, S. S.; Tomasi, J.; Cossi, M.; Rega, N.; Millam, J. M.; Klene, M.; Knox, J. E.; Cross, J. B.; Bakken, V.; Adamo, C.; Jaramillo, J.; Gomperts, R.; Stratmann, R. E.; Yazyev, O.; Austin, A. J.; Cammi, R.; Pomelli, C.; Ochterski, J. W.; Martin, R. L.; Morokuma, K.; Zakrzewski, V. G.; Voth, G. A.; Salvador, P.; Dannenberg, J. J.; Dapprich, S.; Daniels, A. D.; Farkas, O.; Foresman, J. B.; Ortiz, J. V.; Cioslowski, J.; Fox, D. J. Gaussian 16, Revision C.01, Gaussian, Inc.: Wallingford CT, 2016. 


\section{3. ${ }^{1} \mathrm{H}$ and ${ }^{13} \mathrm{C}$ NMR Spectra}

${ }^{1} \mathrm{H}$ NMR spectrum of $\mathbf{1 a}\left(400 \mathrm{MHz}, \mathrm{CD}_{3} \mathrm{CN}\right)$

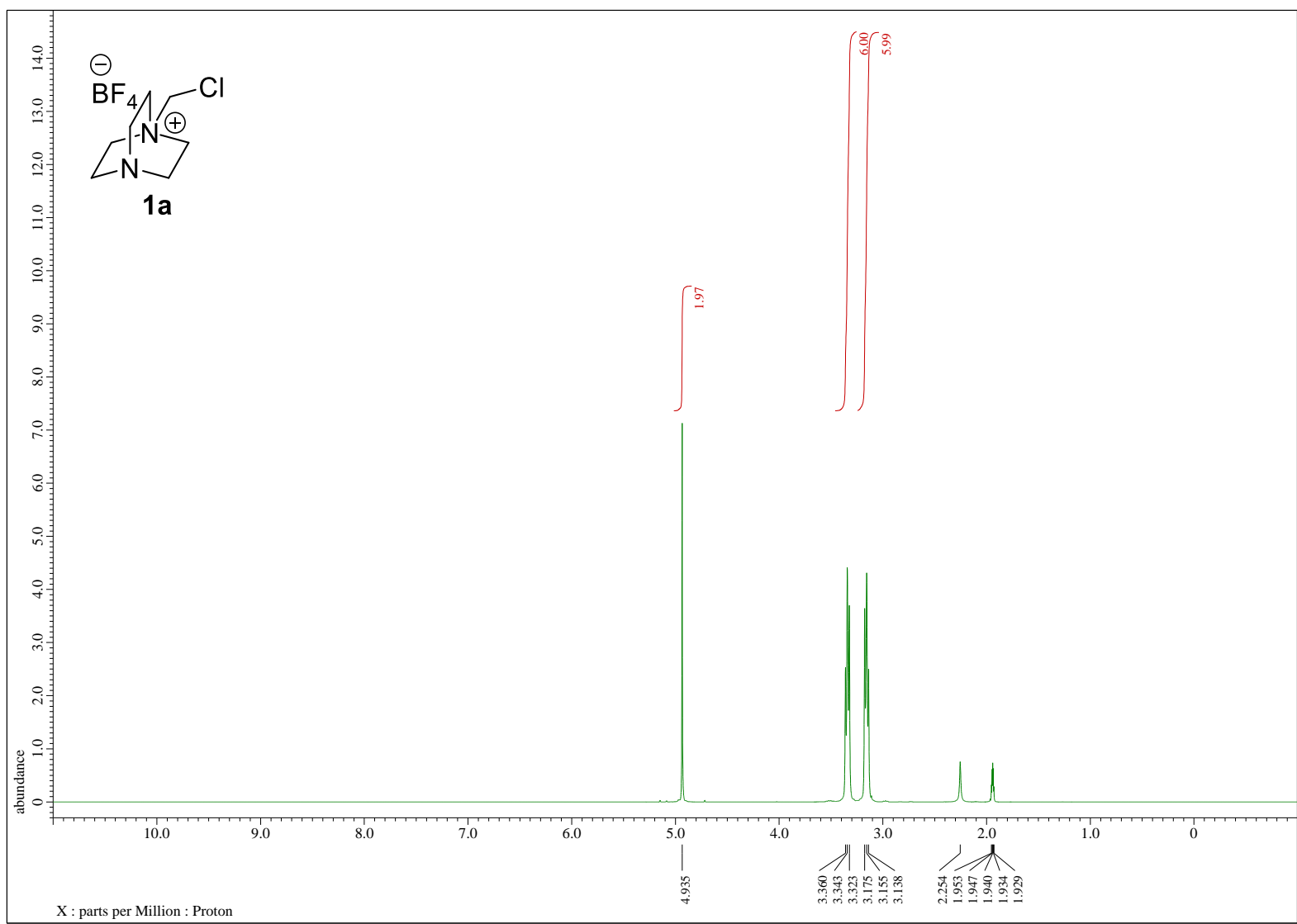

${ }^{13} \mathrm{C}$ NMR spectrum of $\mathbf{1 a}\left(100 \mathrm{MHz}, \mathrm{CD}_{3} \mathrm{CN}\right)$

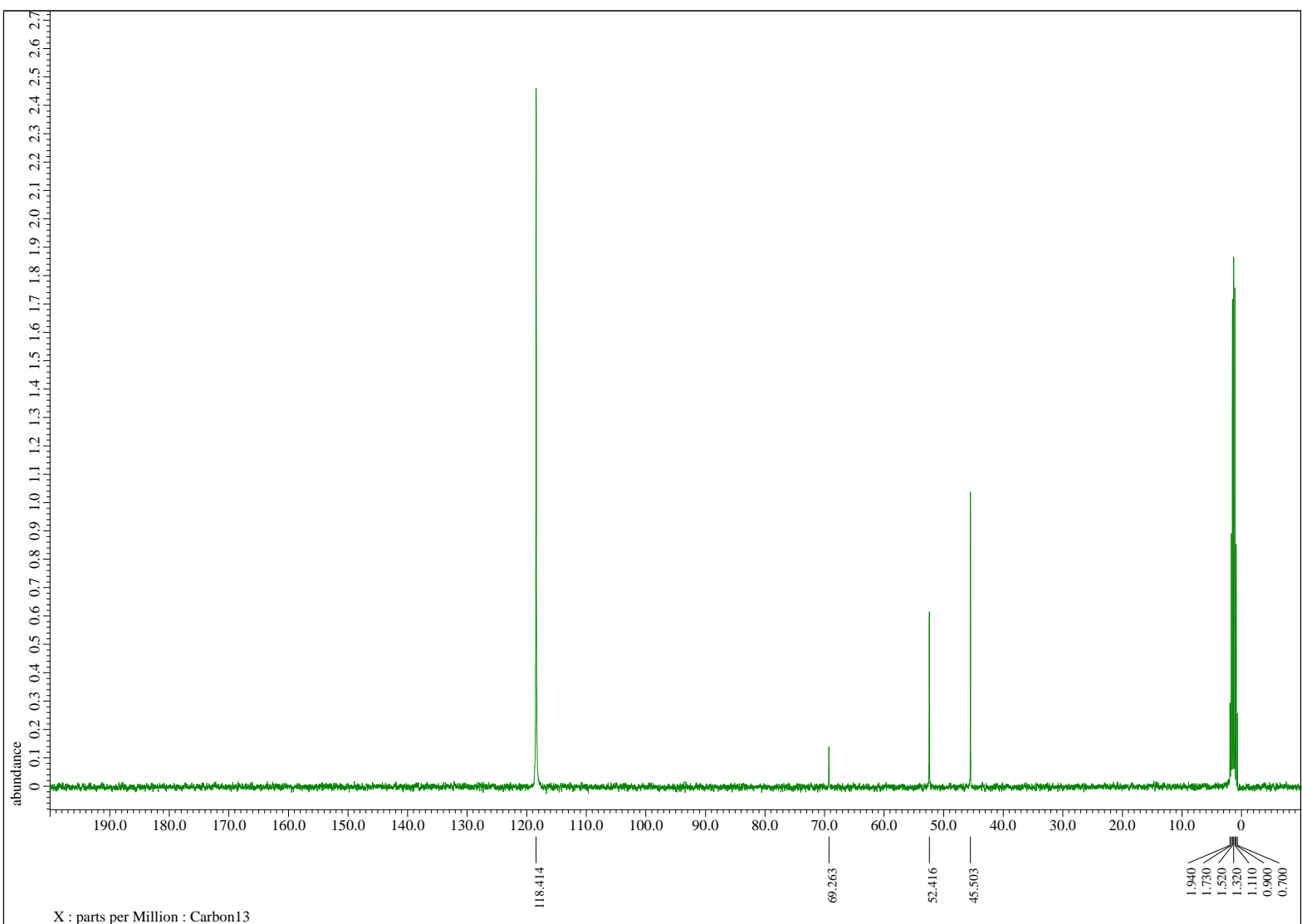


${ }^{1} \mathrm{H}$ NMR spectrum of $\mathbf{1 b}\left(400 \mathrm{MHz}, \mathrm{CD}_{3} \mathrm{CN}\right)$

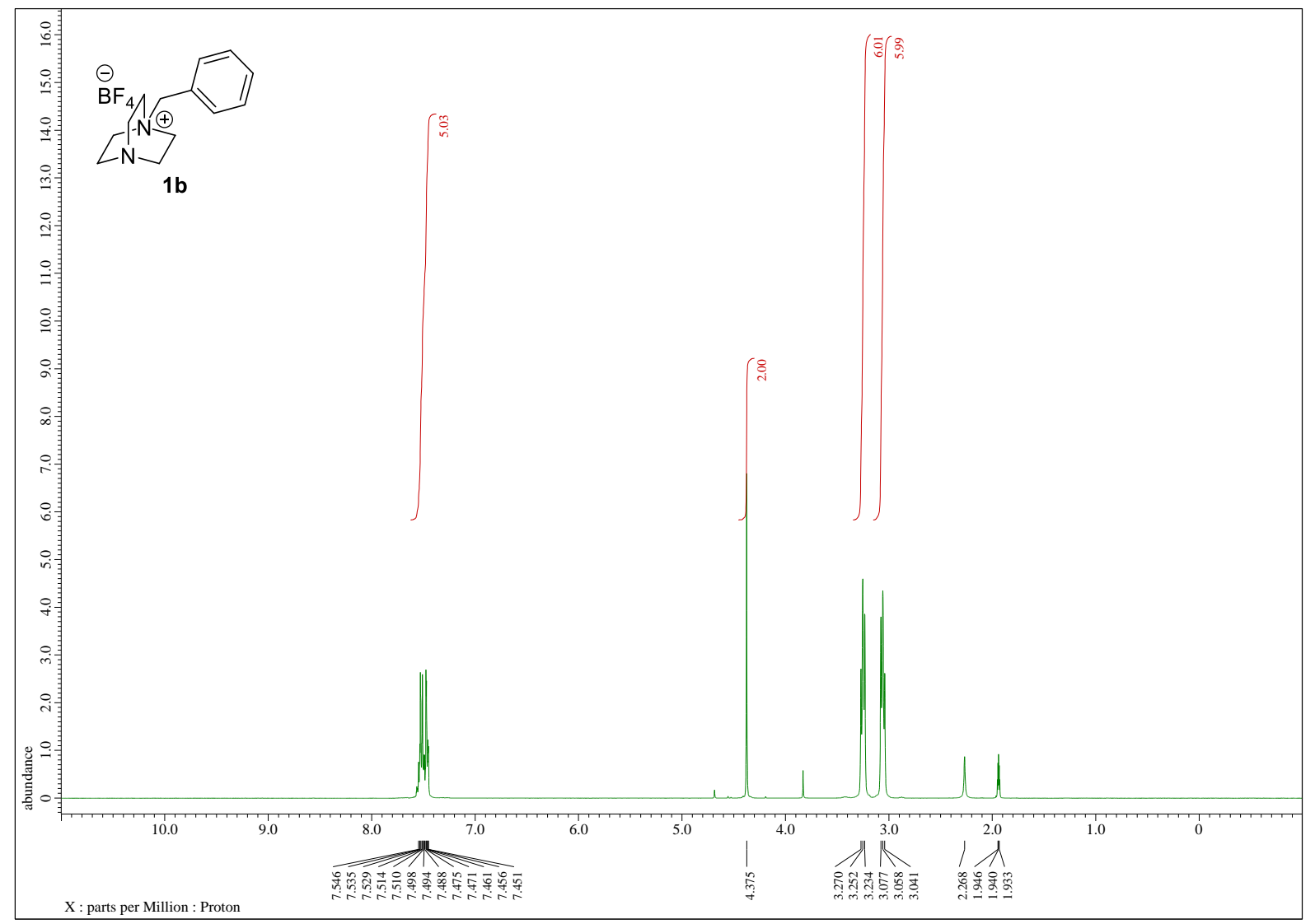

${ }^{13} \mathrm{C}$ NMR spectrum of $\mathbf{1 b}\left(100 \mathrm{MHz}, \mathrm{CD}_{3} \mathrm{CN}\right)$

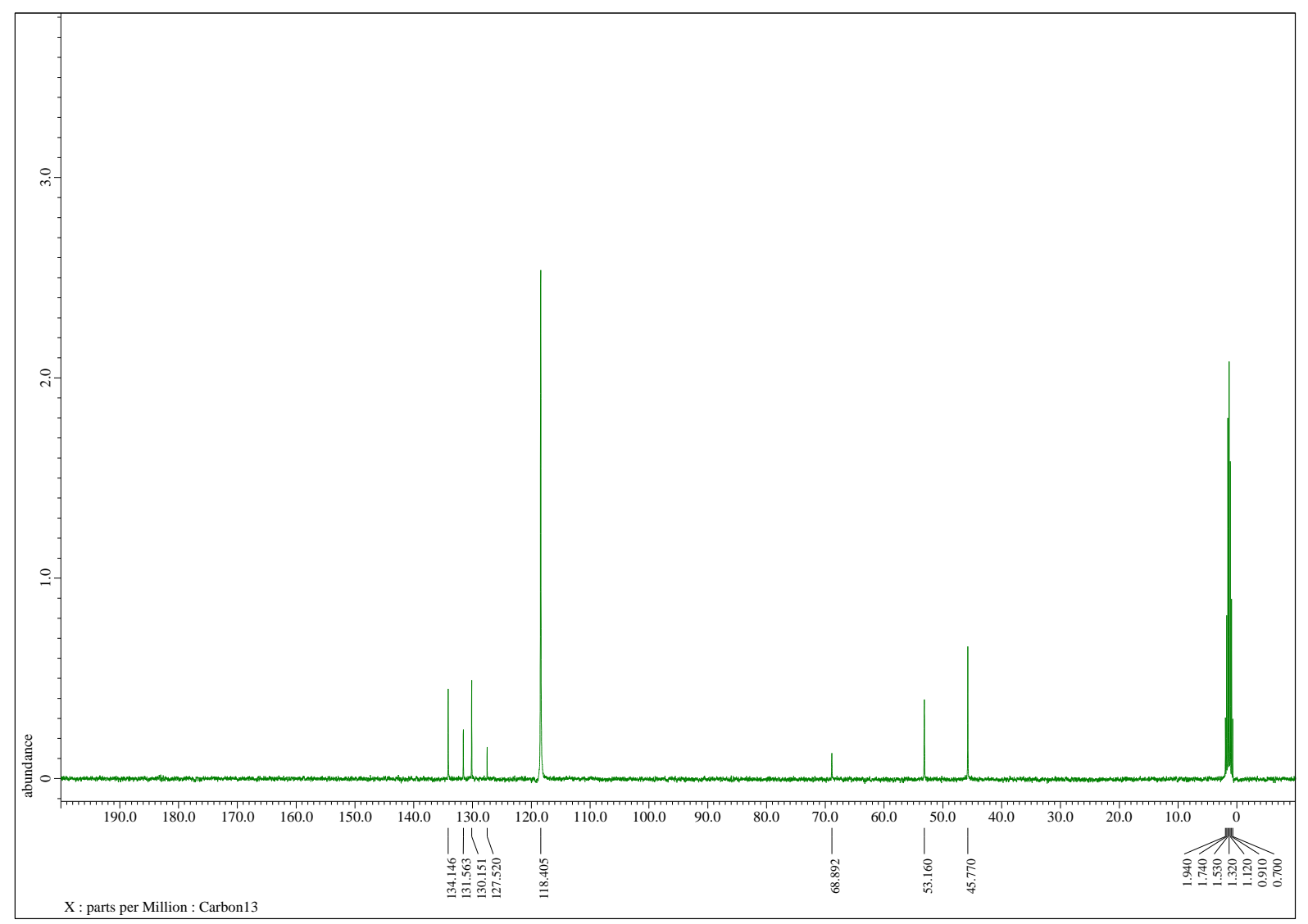


${ }^{1} \mathrm{H}$ NMR spectrum of $\mathbf{1 c}\left(400 \mathrm{MHz}, \mathrm{CD}_{3} \mathrm{CN}\right)$

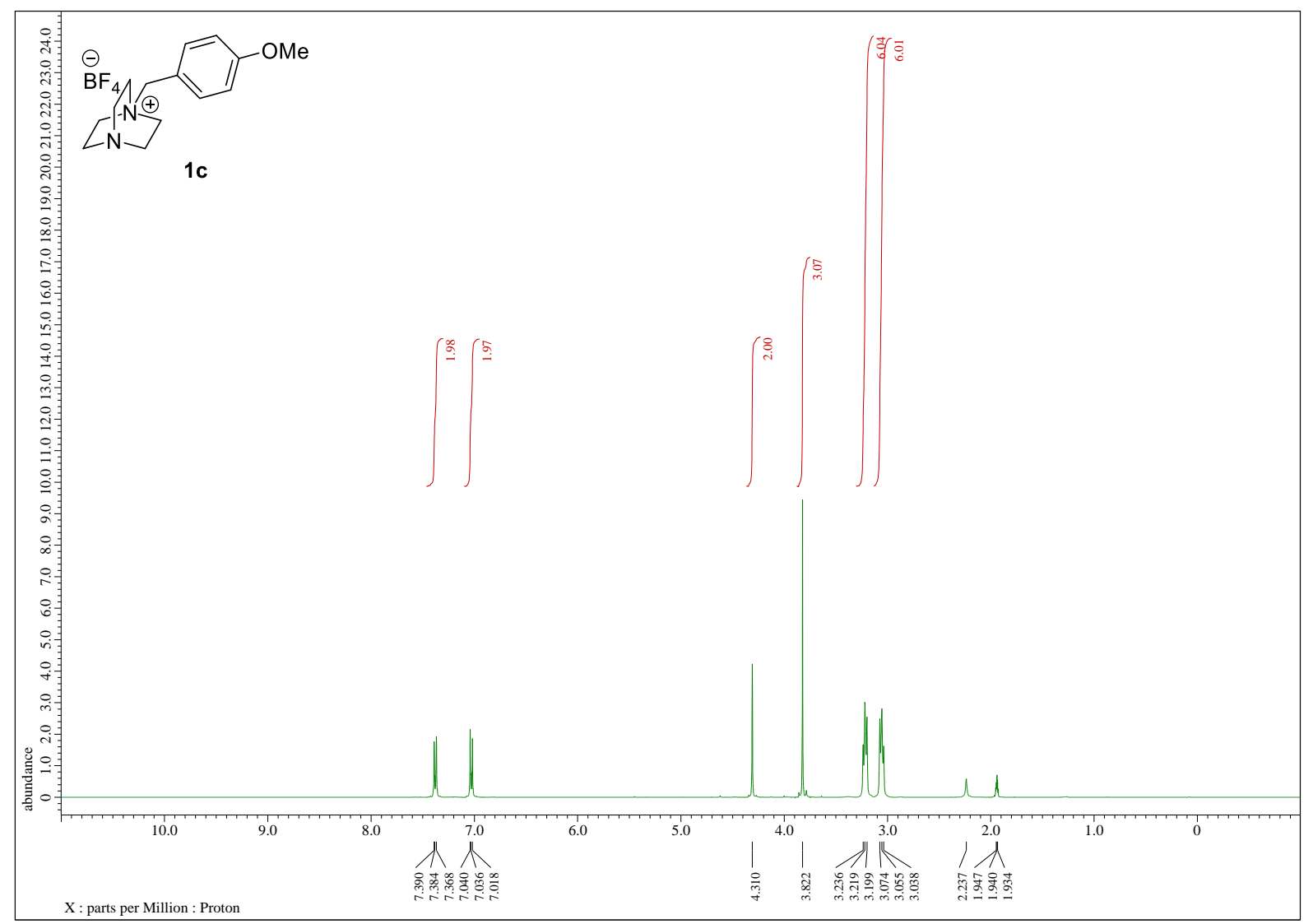

${ }^{13} \mathrm{C}$ NMR spectrum of $1 \mathrm{c}\left(100 \mathrm{MHz}, \mathrm{CD}_{3} \mathrm{CN}\right)$

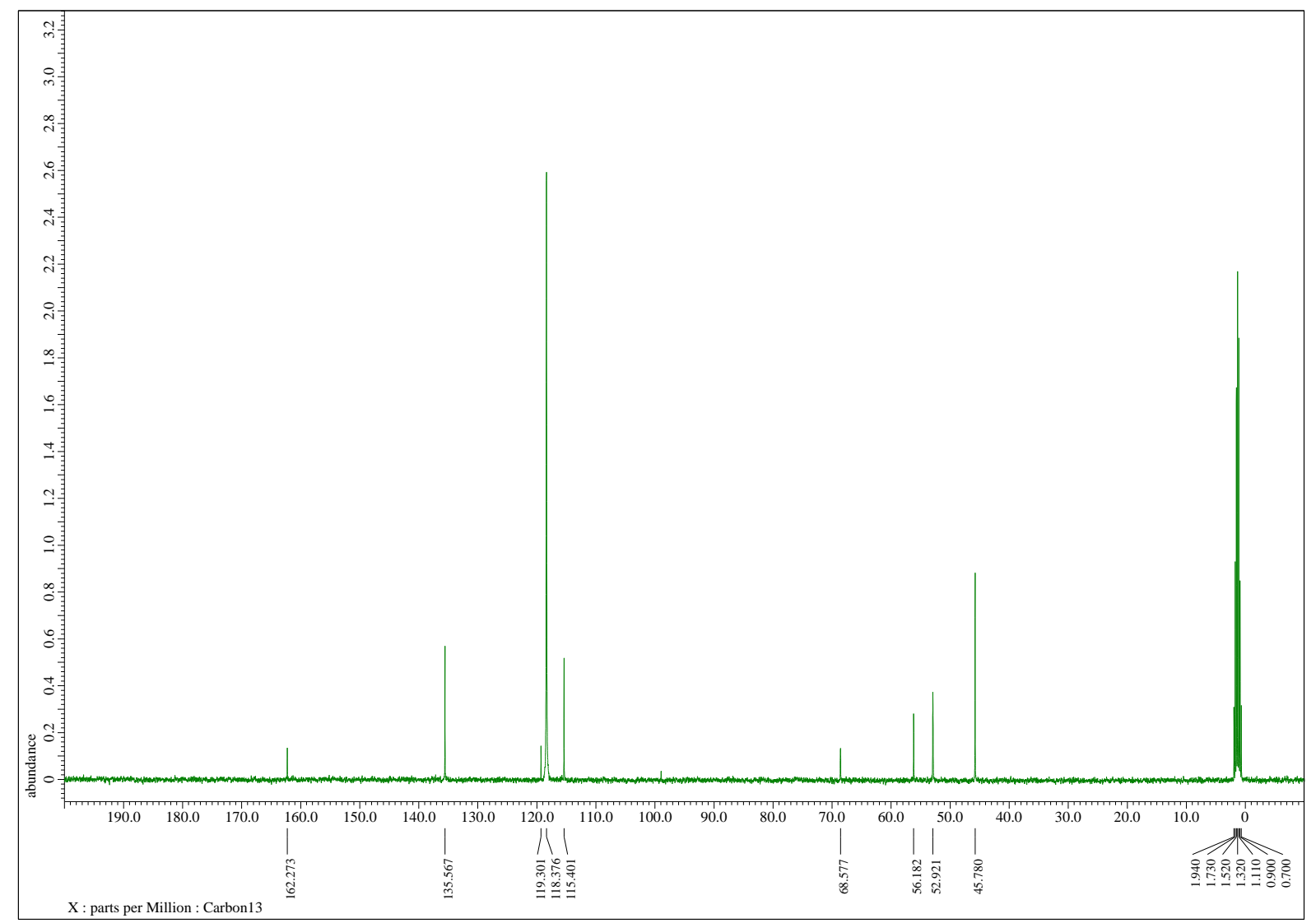


${ }^{1} \mathrm{H}$ NMR spectrum of $\mathbf{1 d}\left(400 \mathrm{MHz}, \mathrm{CD}_{3} \mathrm{CN}\right)$

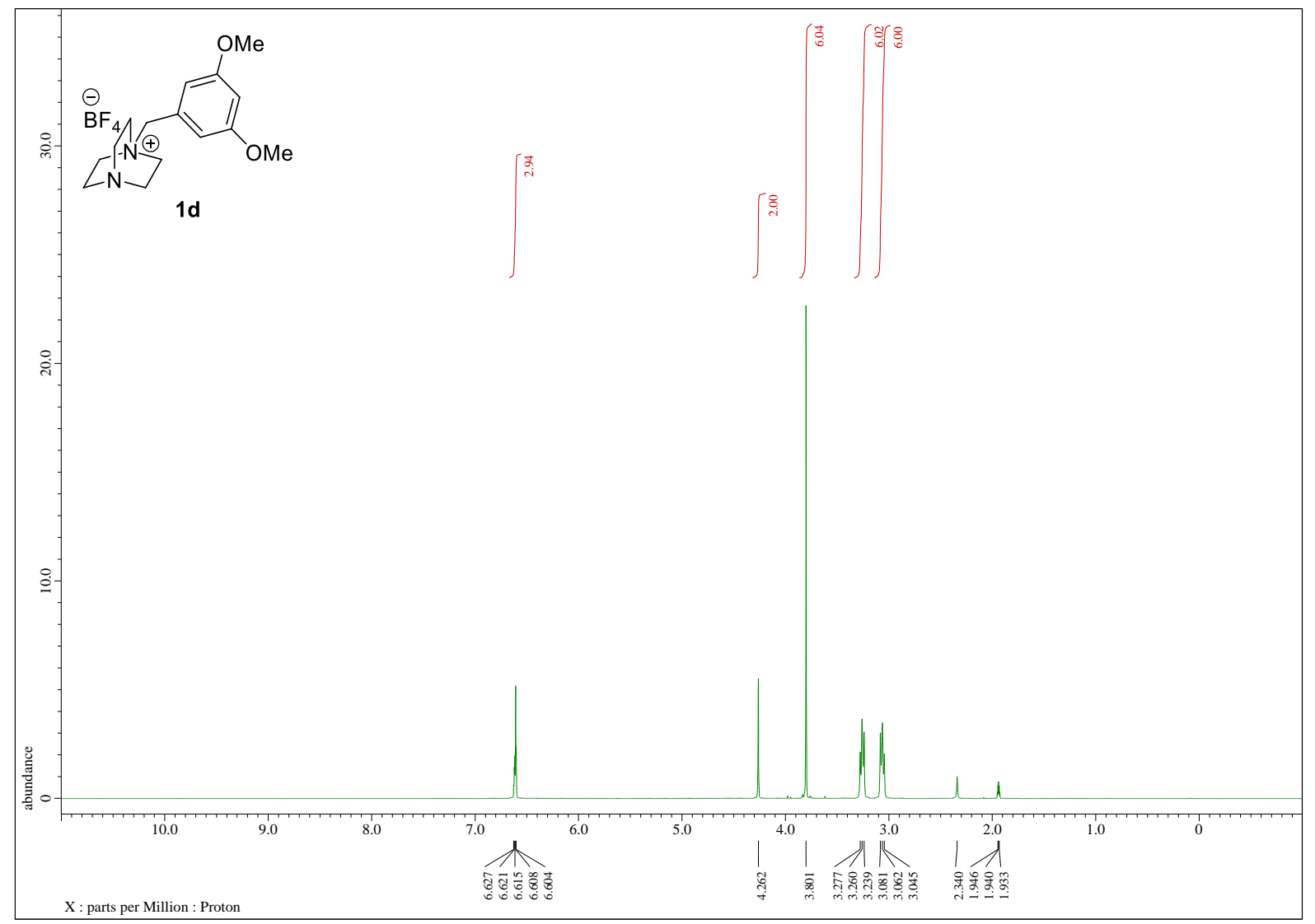

${ }^{13} \mathrm{C}$ NMR spectrum of $\mathbf{1 d}\left(100 \mathrm{MHz}, \mathrm{CD}_{3} \mathrm{CN}\right)$

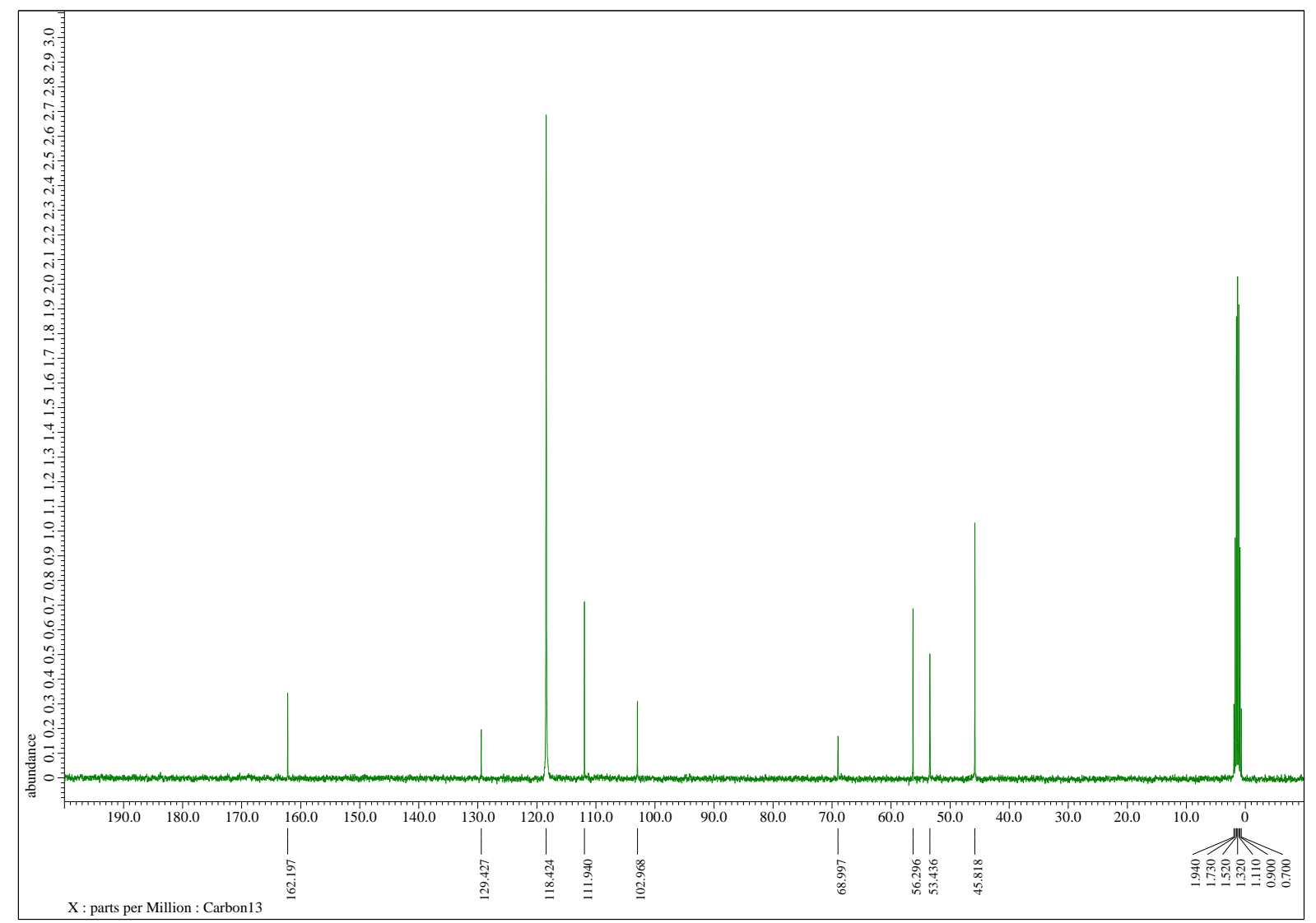


${ }^{1} \mathrm{H}$ NMR spectrum of $\mathbf{1 e}\left(400 \mathrm{MHz}, \mathrm{CD}_{3} \mathrm{CN}\right)$

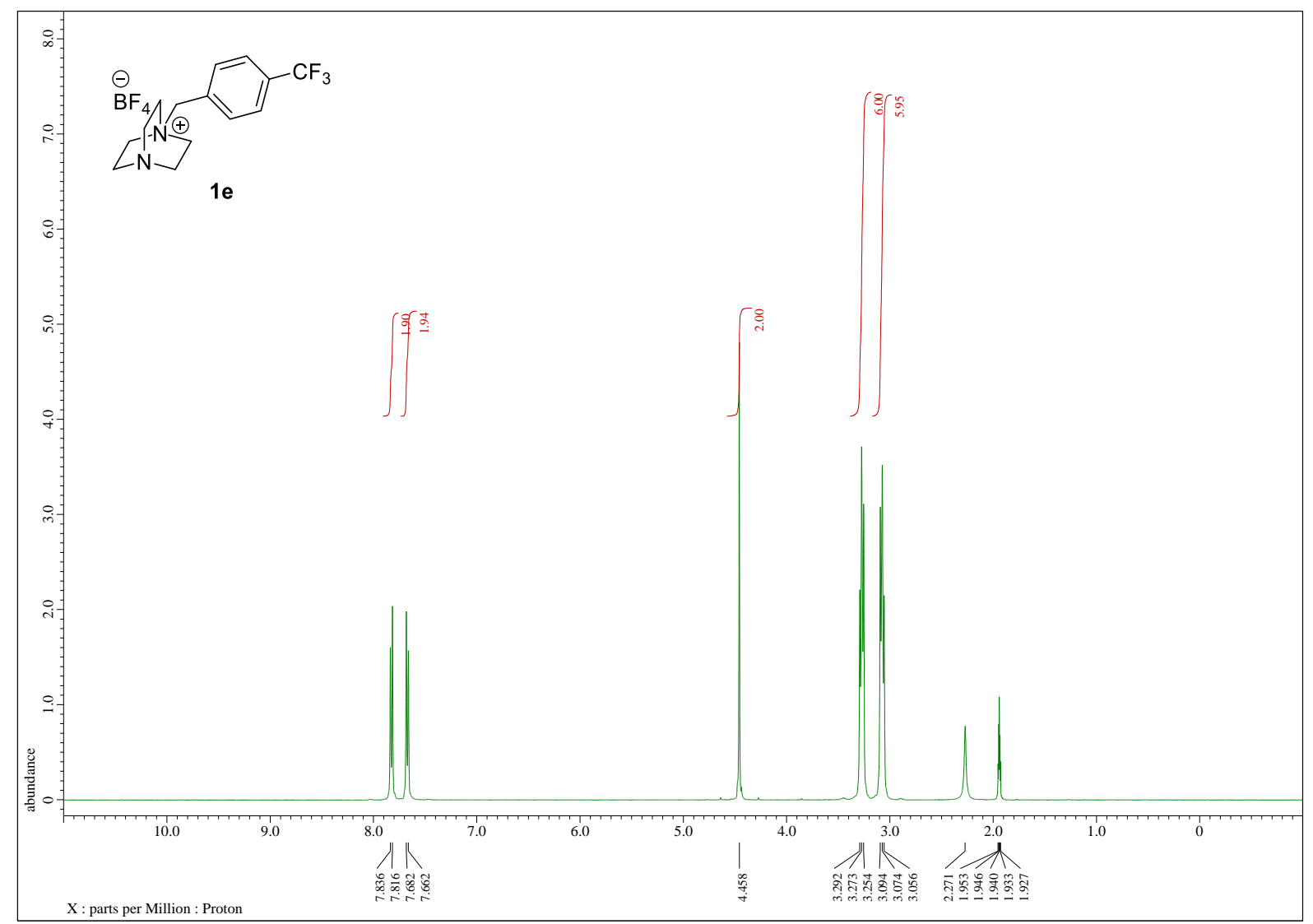

${ }^{13} \mathrm{C}$ NMR spectrum of $1 \mathbf{e}\left(100 \mathrm{MHz}, \mathrm{CD}_{3} \mathrm{CN}\right)$

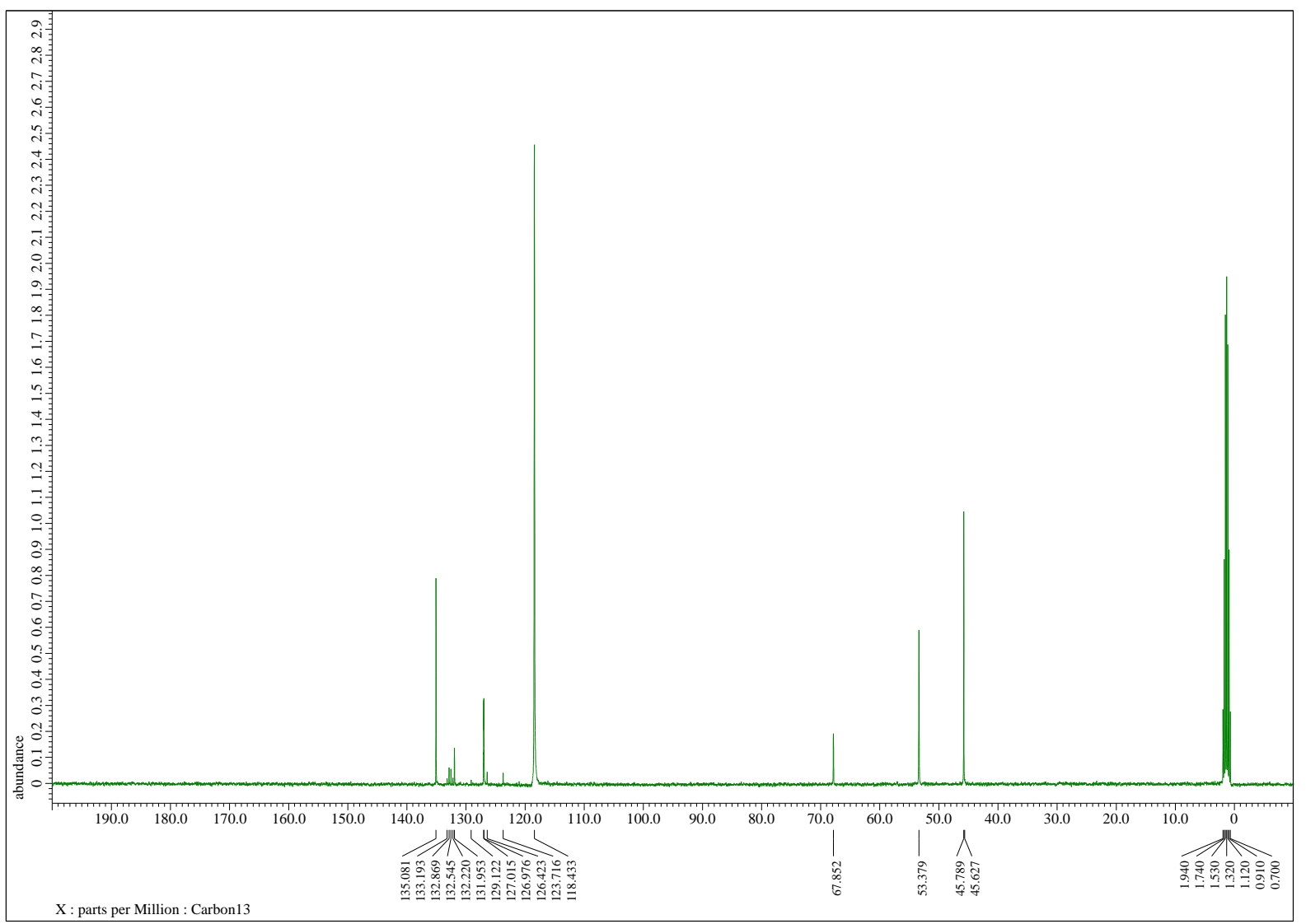


${ }^{1} \mathrm{H}$ NMR spectrum of $\mathbf{1 f}\left(400 \mathrm{MHz}, \mathrm{CD}_{3} \mathrm{CN}\right)$

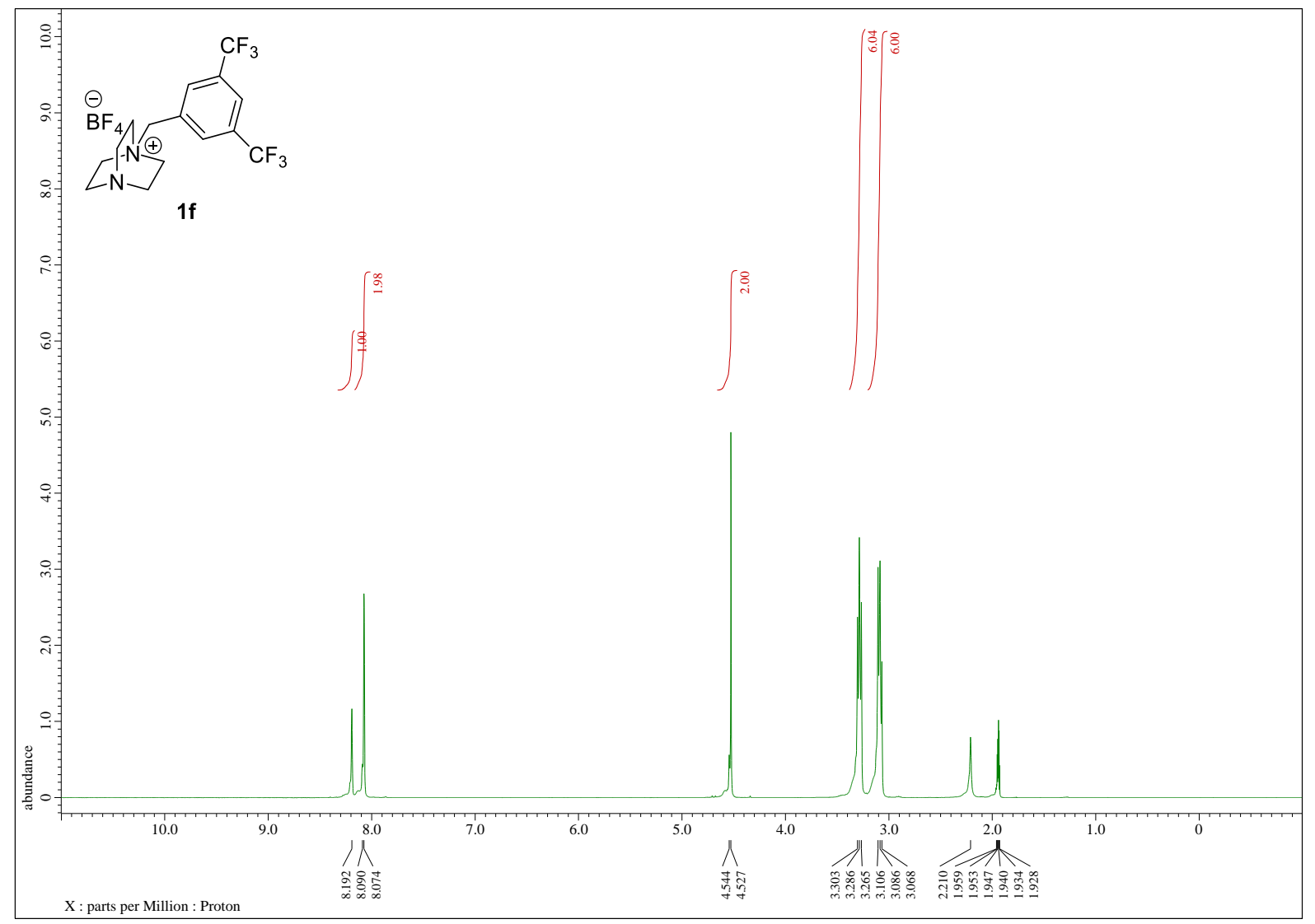

${ }^{13} \mathrm{C}$ NMR spectrum of $\mathbf{1 f}\left(100 \mathrm{MHz}, \mathrm{CD}_{3} \mathrm{CN}\right)$

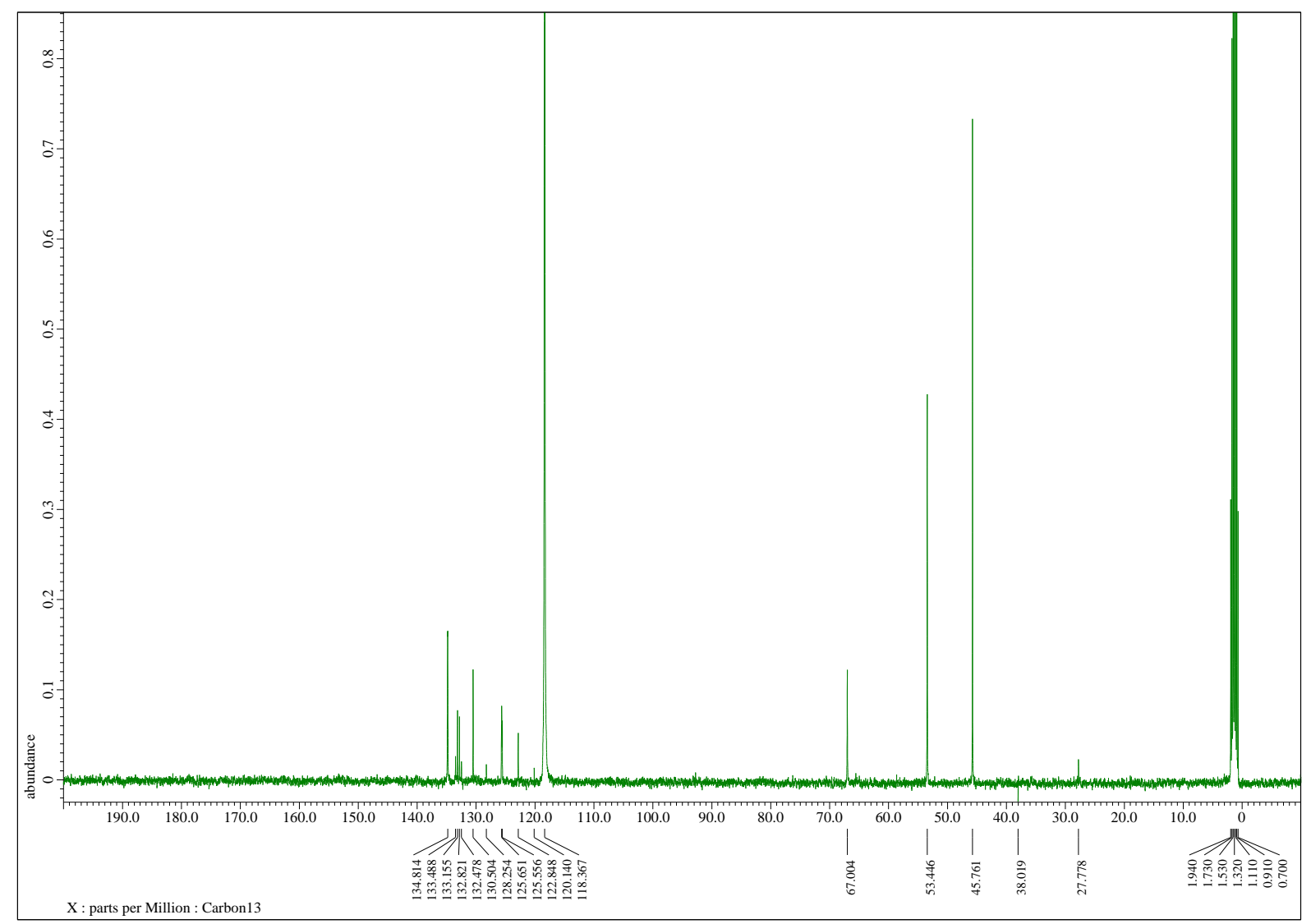


${ }^{1} \mathrm{H}$ NMR spectrum of $\mathbf{1 g}\left(400 \mathrm{MHz}, \mathrm{CD}_{3} \mathrm{CN}\right)$

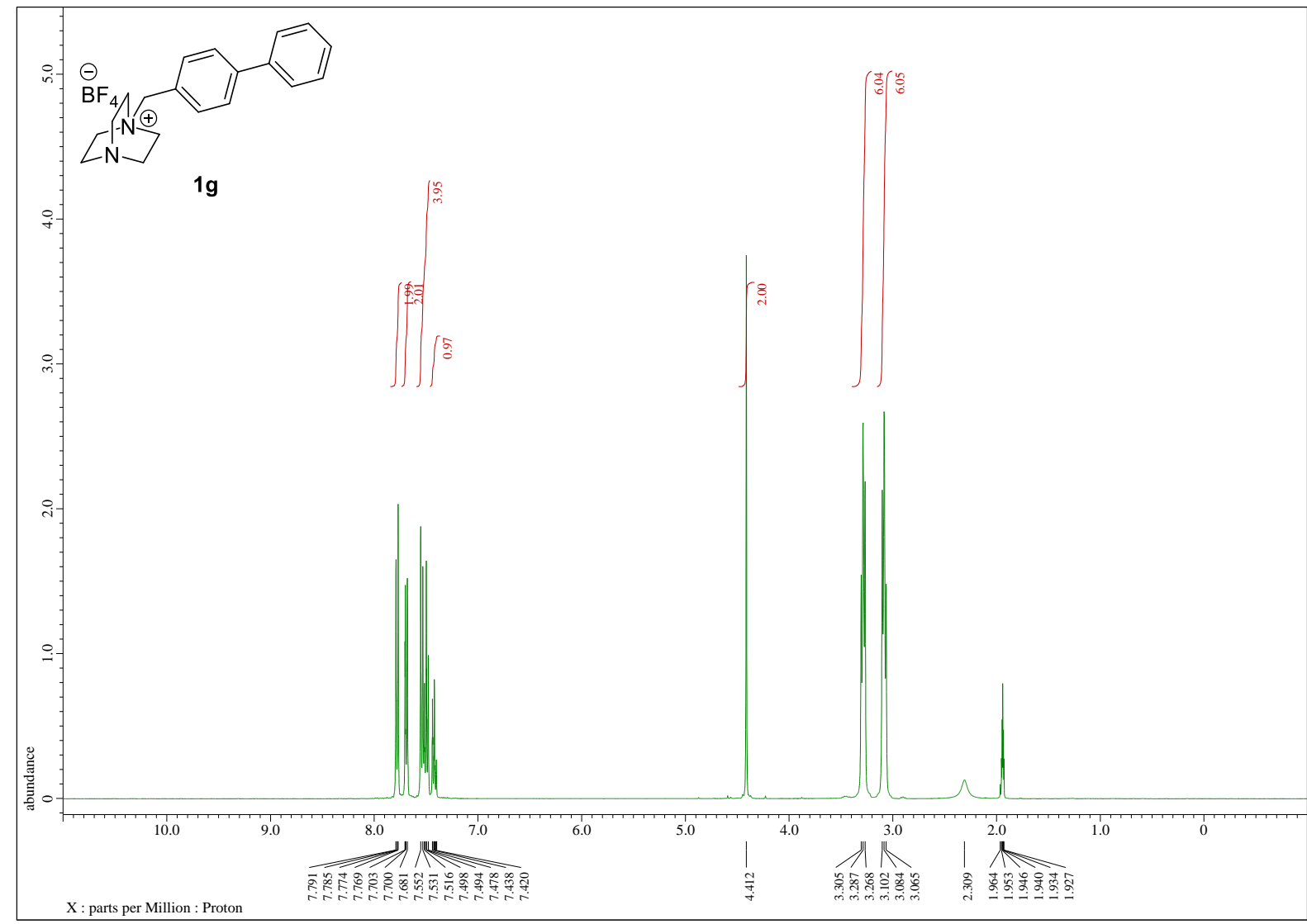

${ }^{13} \mathrm{C}$ NMR spectrum of $\mathbf{1 g}\left(100 \mathrm{MHz}, \mathrm{CD}_{3} \mathrm{CN}\right)$

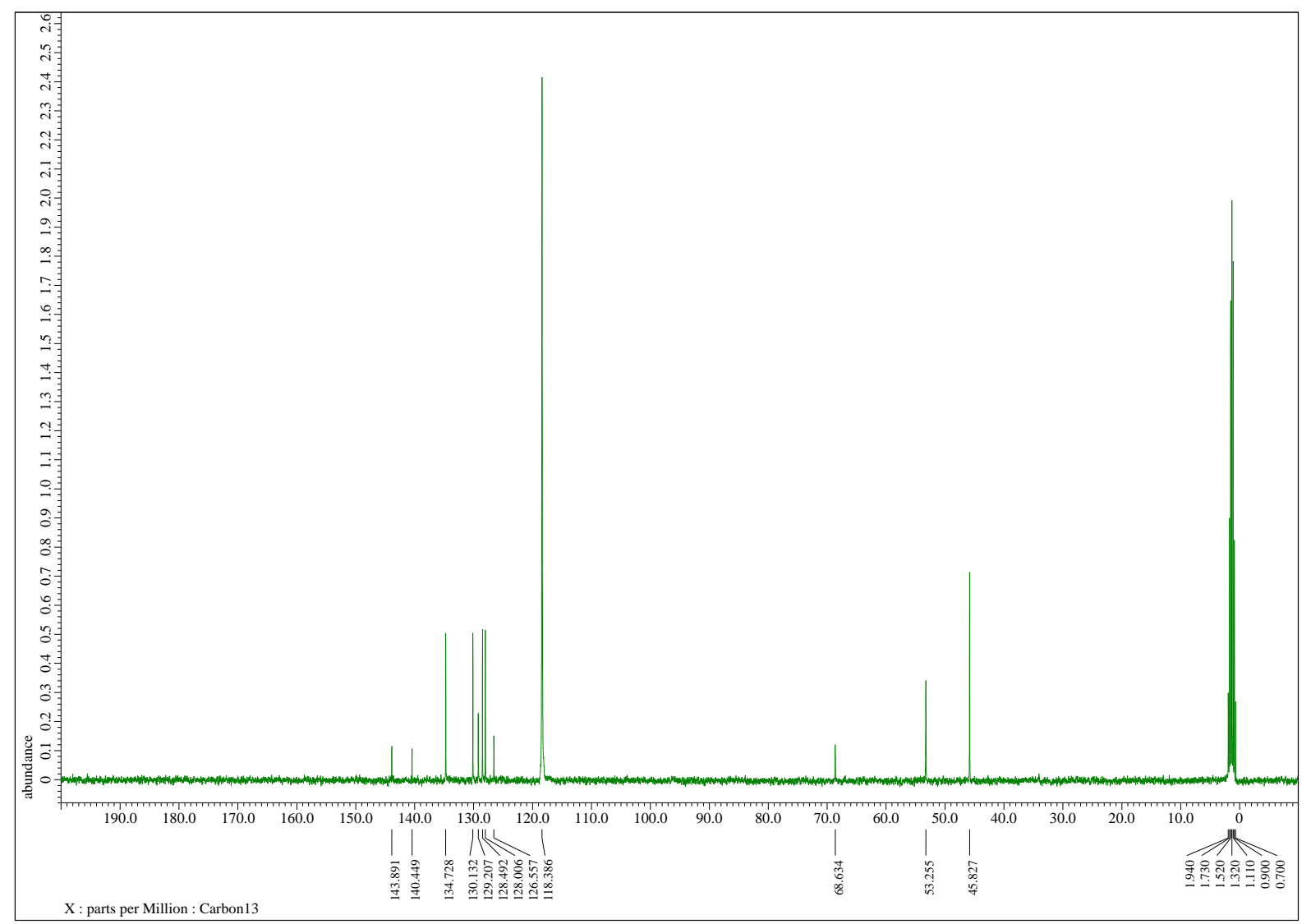


${ }^{1} \mathrm{H}$ NMR spectrum of $\mathbf{1 h}\left(400 \mathrm{MHz}, \mathrm{CD}_{3} \mathrm{CN}\right)$

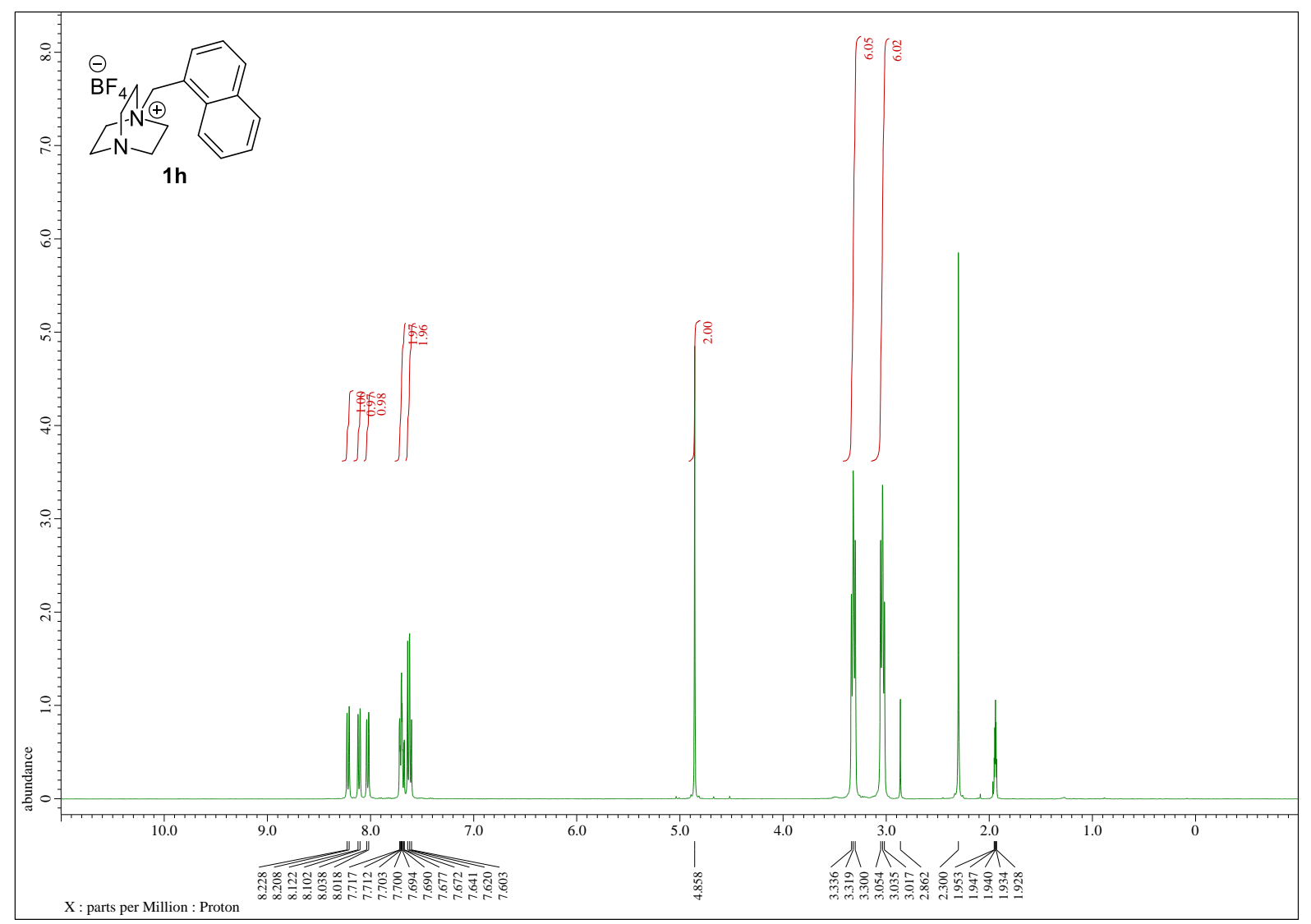

${ }^{13} \mathrm{C}$ NMR spectrum of $\mathbf{1 h}\left(100 \mathrm{MHz}, \mathrm{CD}_{3} \mathrm{CN}\right)$

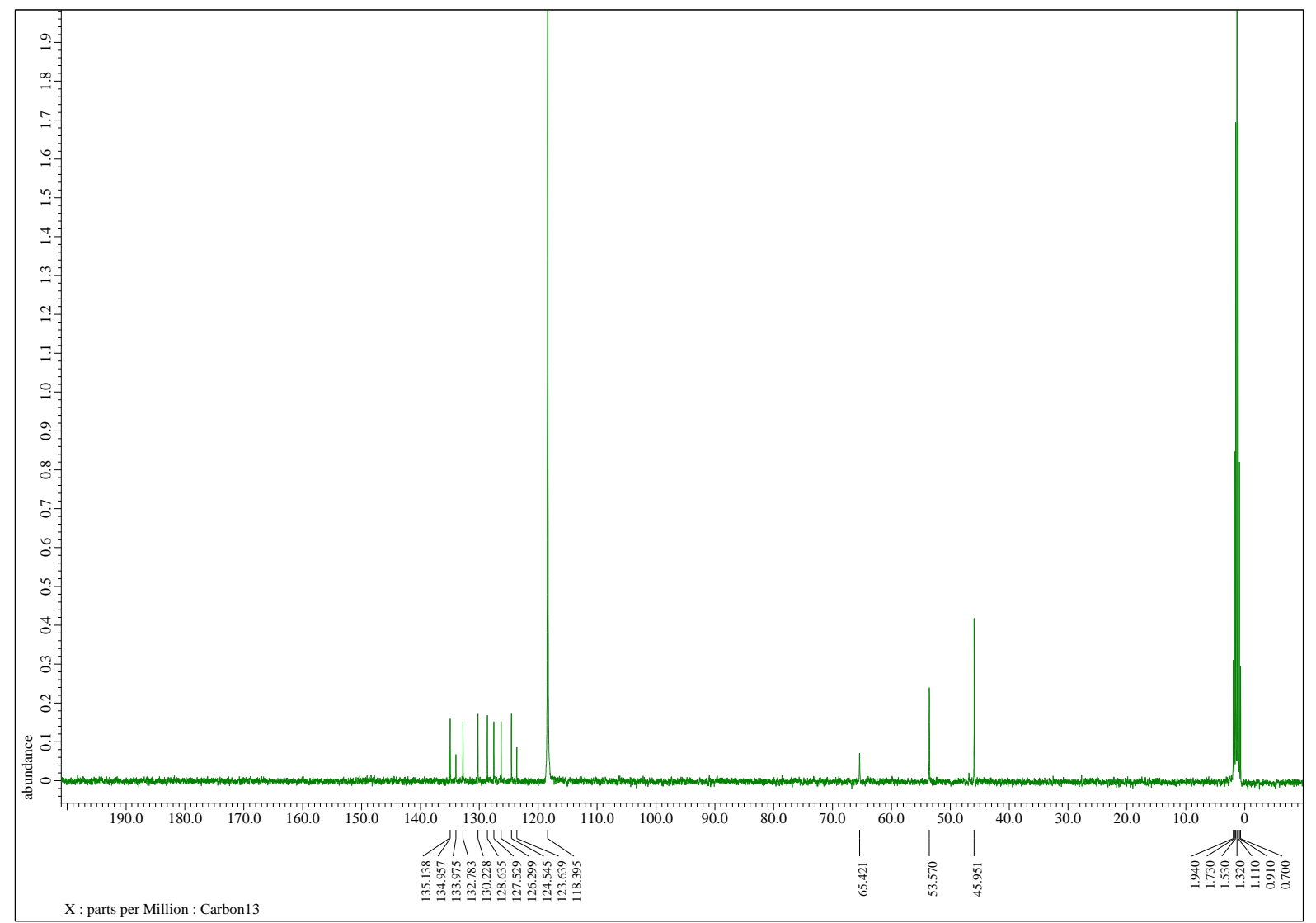


${ }^{1} \mathrm{H}$ NMR spectrum of $\mathbf{1 i}\left(400 \mathrm{MHz}, \mathrm{CD}_{3} \mathrm{CN}\right.$ )

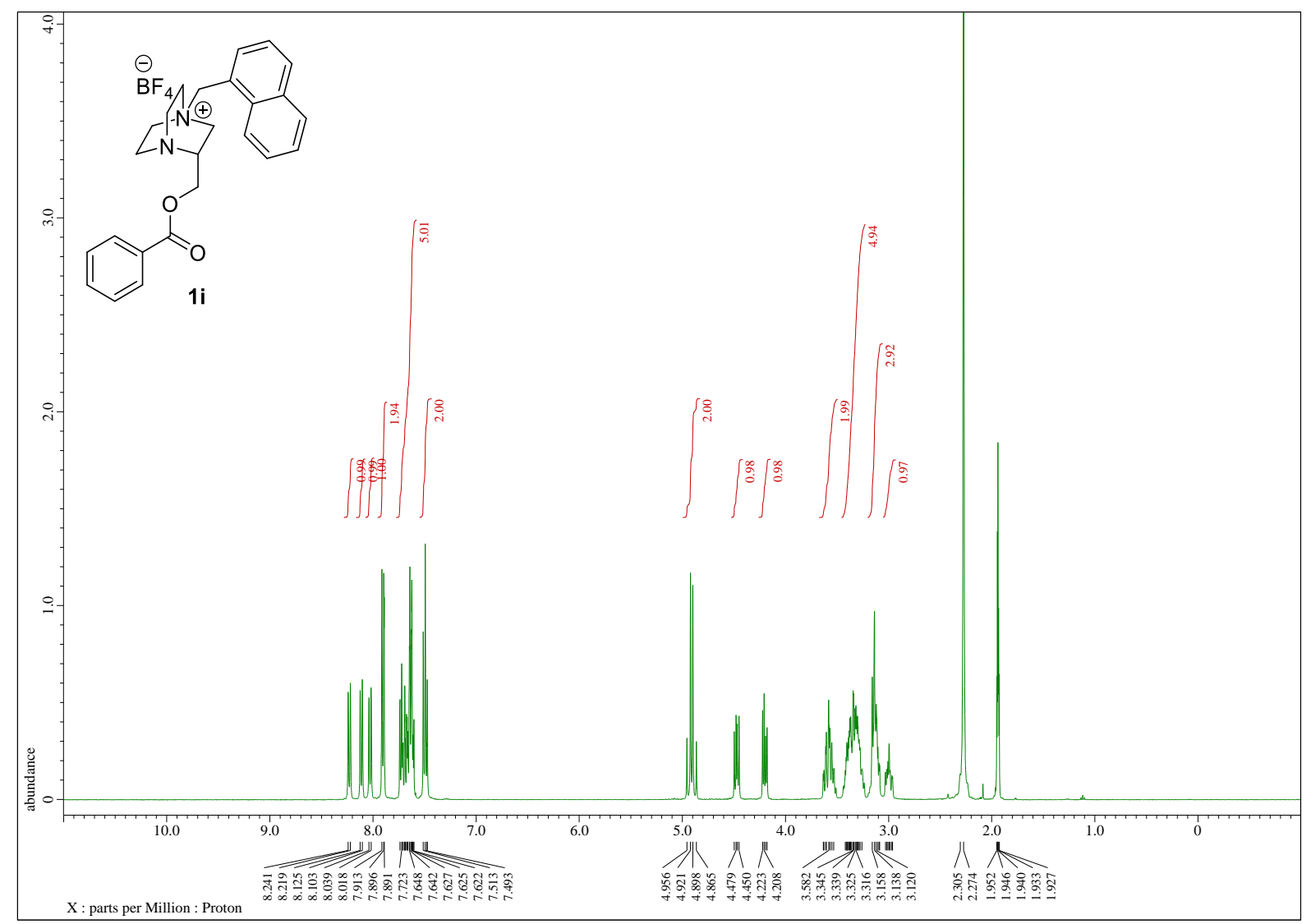

${ }^{13} \mathrm{C}$ NMR spectrum of $\mathbf{1 i}\left(100 \mathrm{MHz}, \mathrm{CD}_{3} \mathrm{CN}\right)$

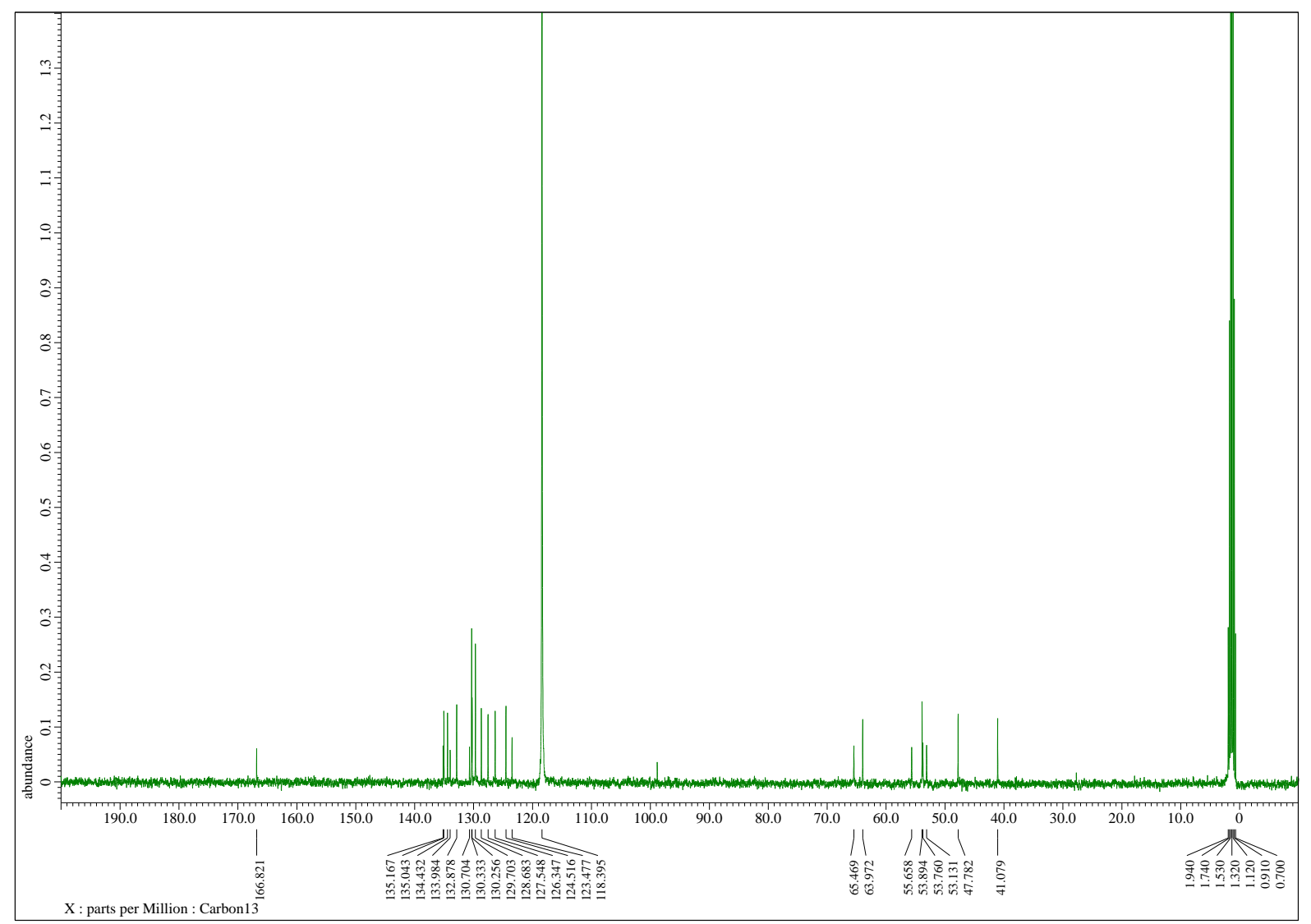


${ }^{1} \mathrm{H}$ NMR spectrum of $\mathbf{1 j}$ (400 MHz, $\mathrm{CD}_{3} \mathrm{CN}$ )

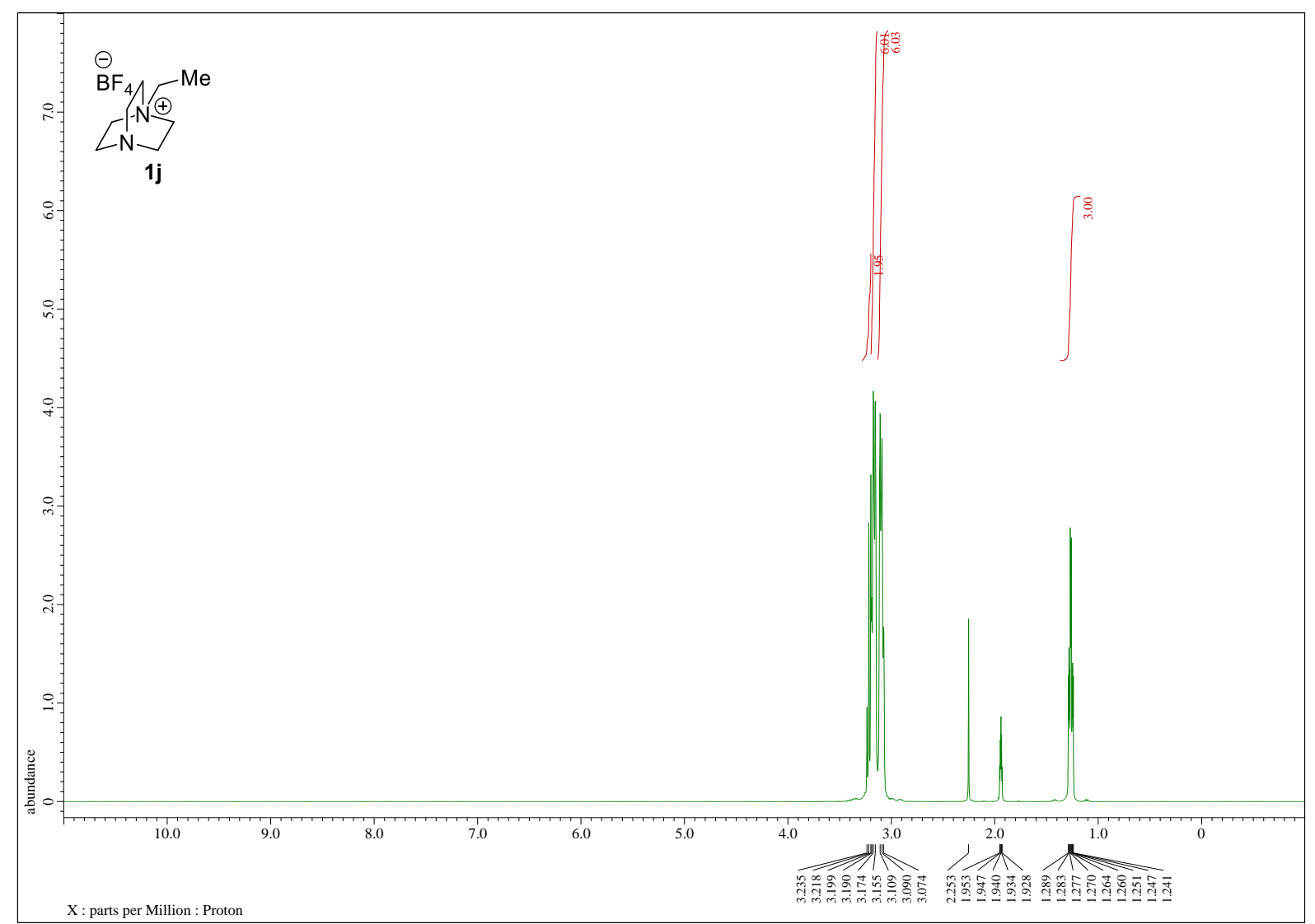

${ }^{13} \mathrm{C}$ NMR spectrum of $\mathbf{1 j}\left(100 \mathrm{MHz}, \mathrm{CD}_{3} \mathrm{CN}\right)$

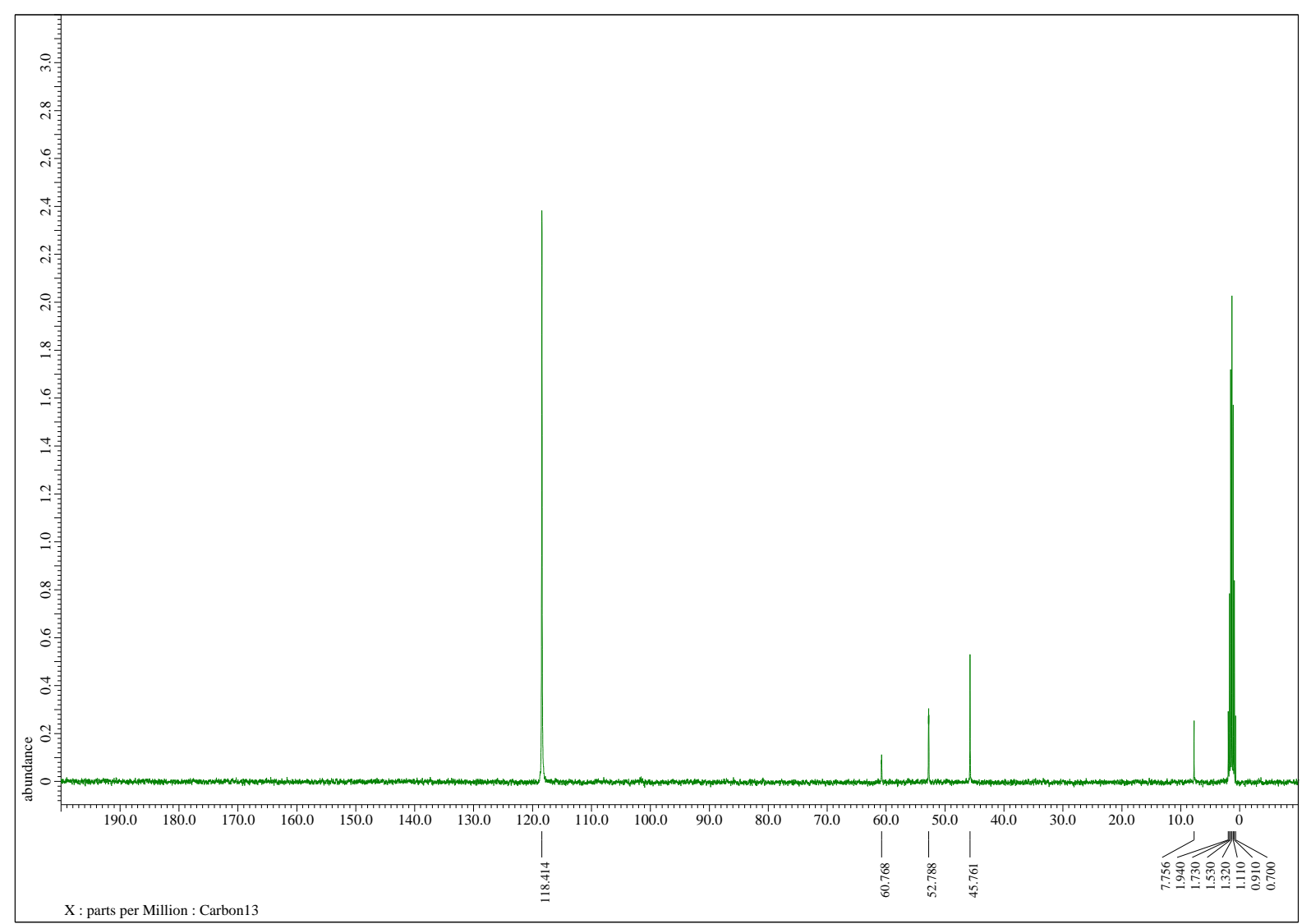


${ }^{1} \mathrm{H}$ NMR spectrum of $\mathbf{1 k}\left(400 \mathrm{MHz}, \mathrm{CD}_{3} \mathrm{CN}\right)$

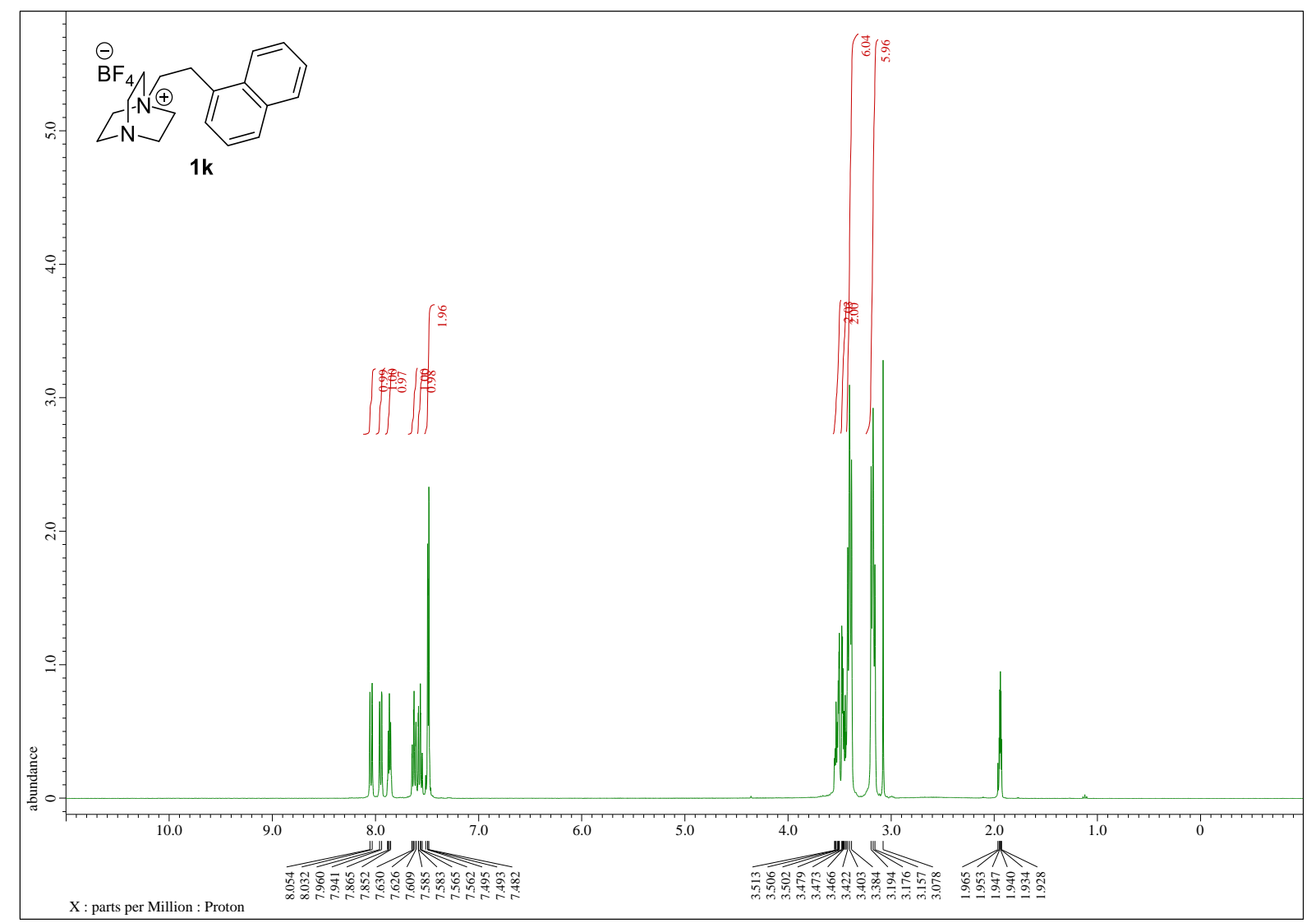

${ }^{13} \mathrm{C}$ NMR spectrum of $\mathbf{1 k}\left(100 \mathrm{MHz}, \mathrm{CD}_{3} \mathrm{CN}\right)$

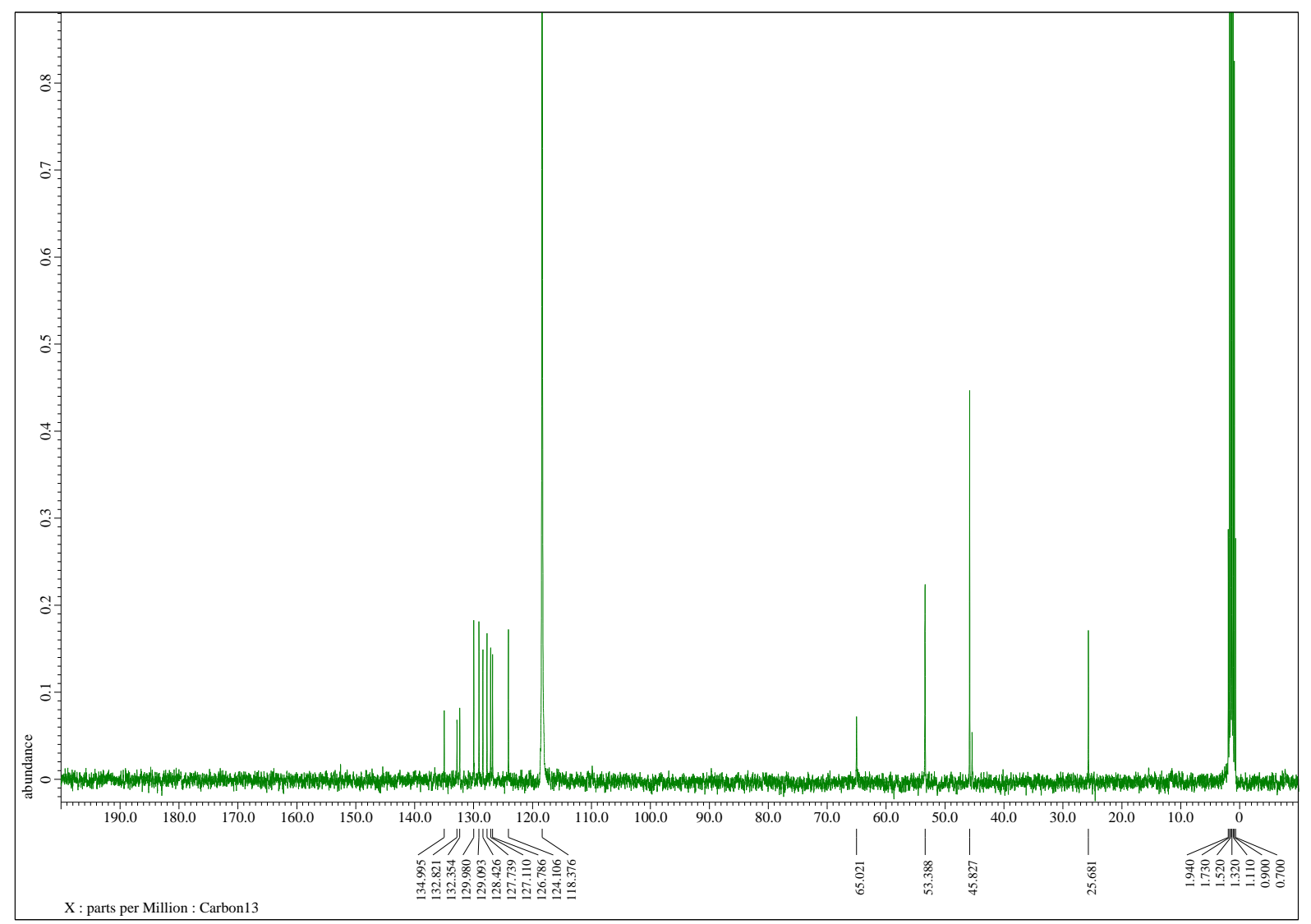


${ }^{1} \mathrm{H} \mathrm{NMR}$ spectrum of $3\left(400 \mathrm{MHz}, \mathrm{CDCl}_{3}\right)$

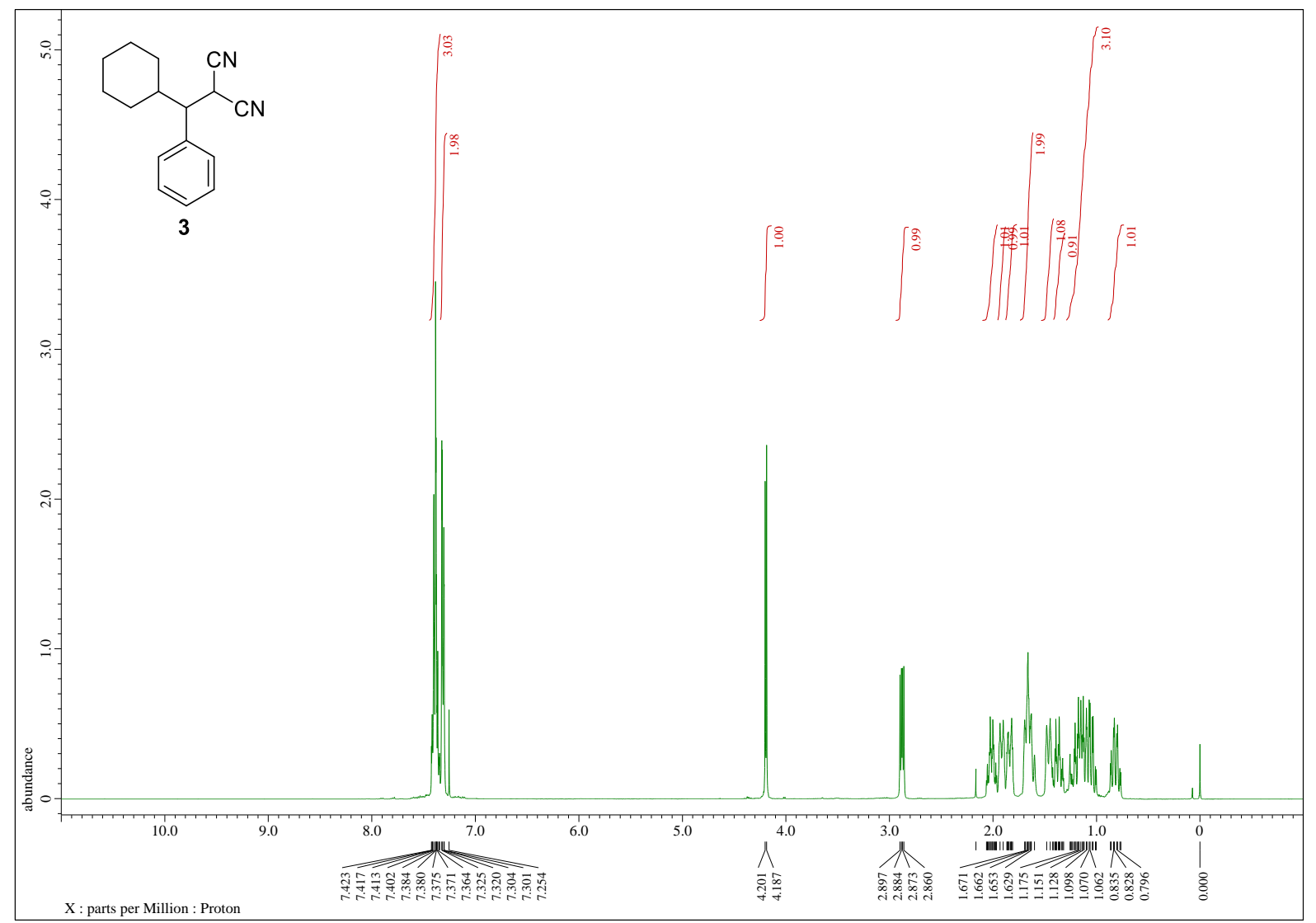

${ }^{13} \mathrm{C}$ NMR spectrum of $3\left(100 \mathrm{MHz}, \mathrm{CDCl}_{3}\right)$

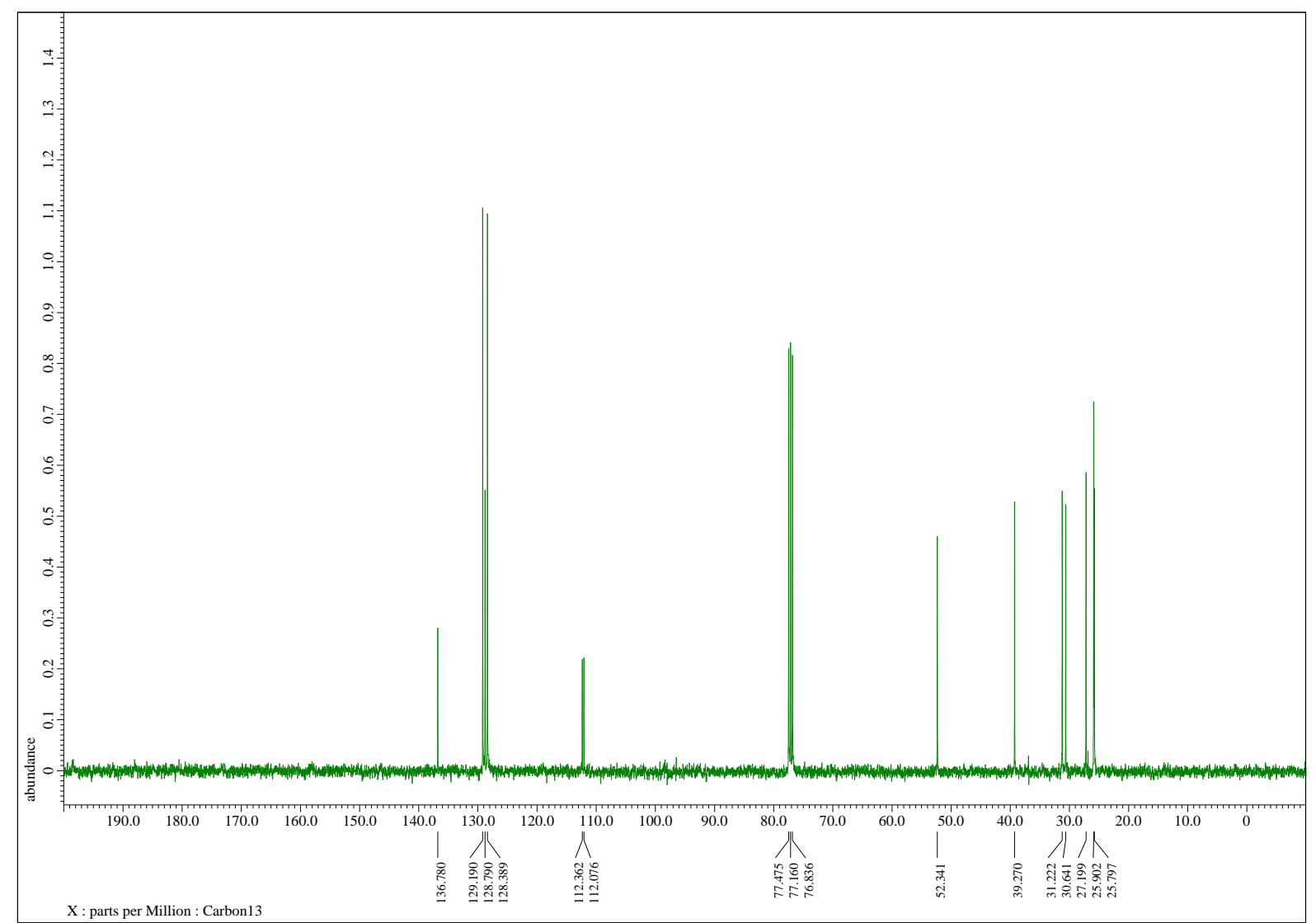


${ }^{1} \mathrm{H}$ NMR spectrum of $4\left(400 \mathrm{MHz}, \mathrm{CDCl}_{3}\right)$

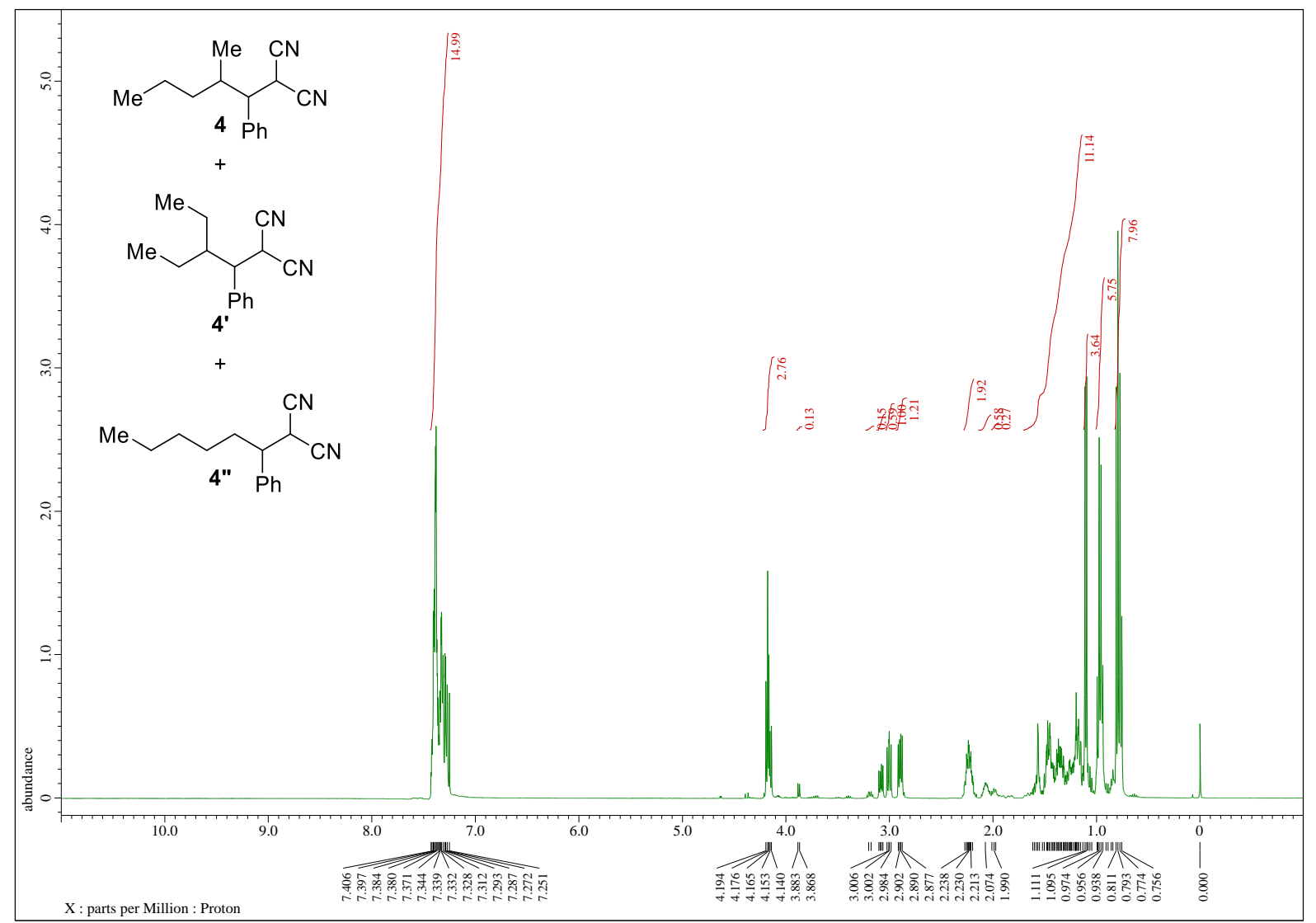

${ }^{13} \mathrm{C}$ NMR spectrum of $4\left(100 \mathrm{MHz}, \mathrm{CDCl}_{3}\right)$

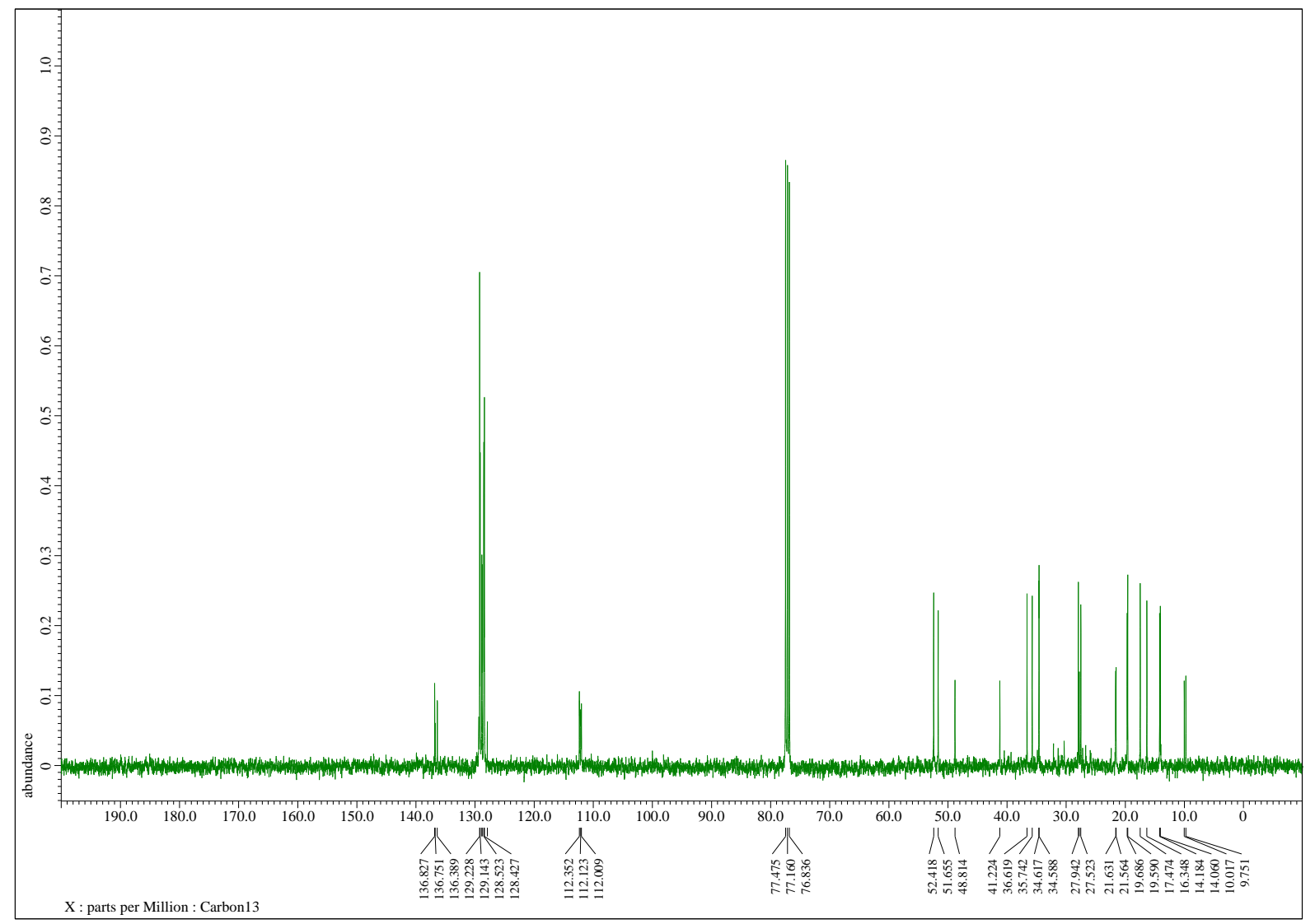


${ }^{1} \mathrm{H}$ NMR spectrum of $5\left(400 \mathrm{MHz}, \mathrm{CDCl}_{3}\right)$

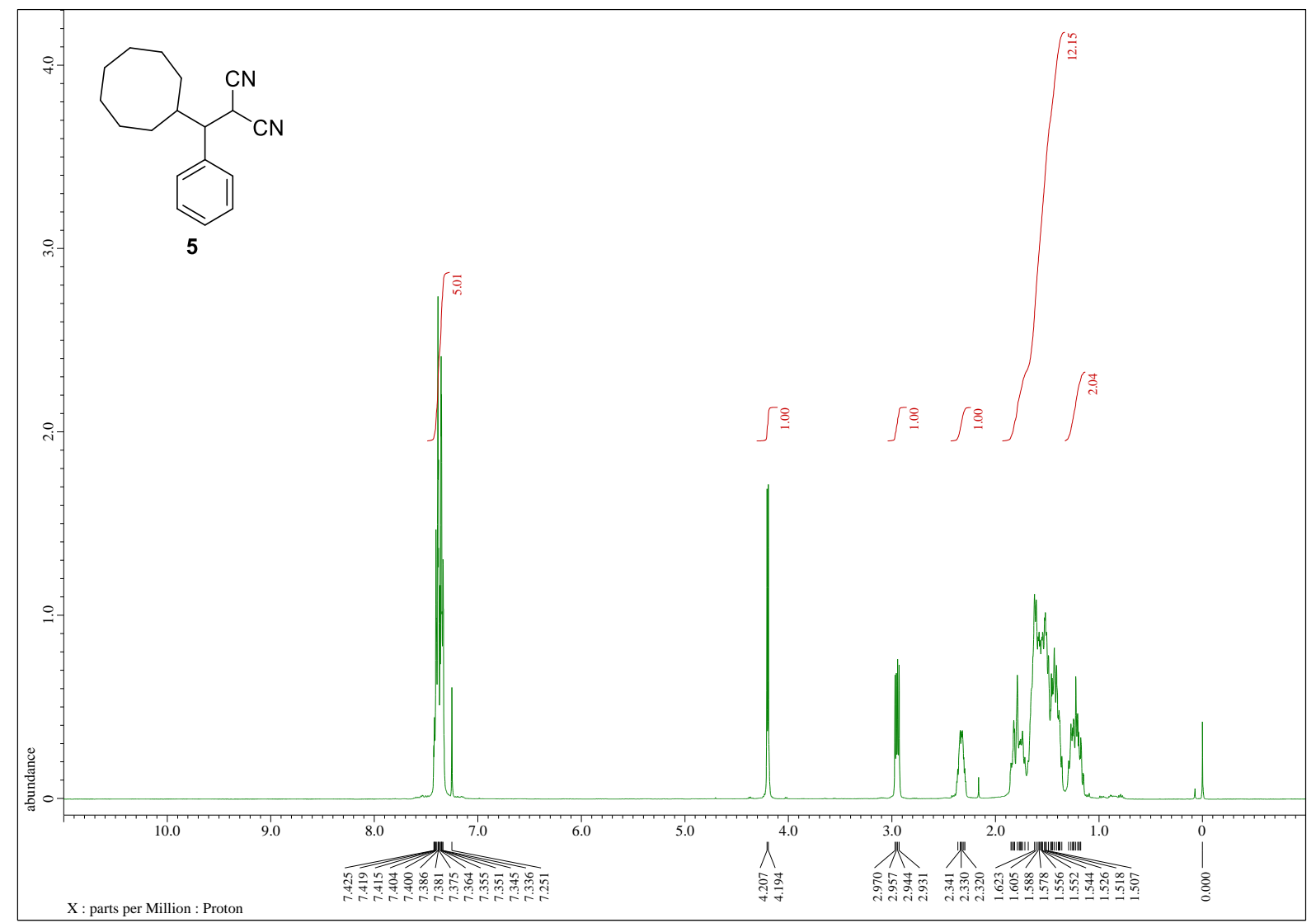

${ }^{13} \mathrm{C}$ NMR spectrum of $5\left(100 \mathrm{MHz}, \mathrm{CDCl}_{3}\right)$

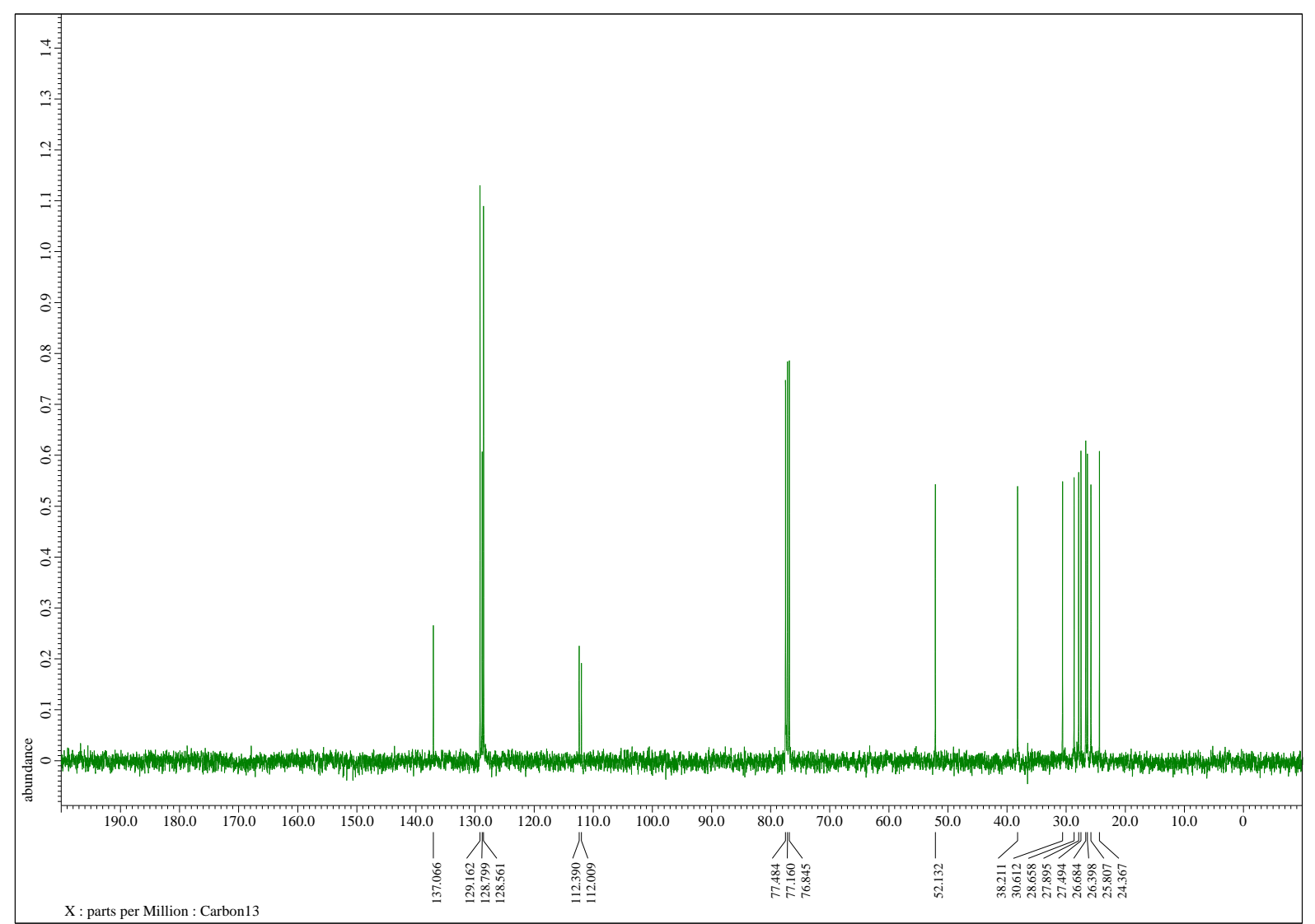


${ }^{1} \mathrm{H}$ NMR spectrum of $6\left(400 \mathrm{MHz}, \mathrm{CDCl}_{3}\right)$

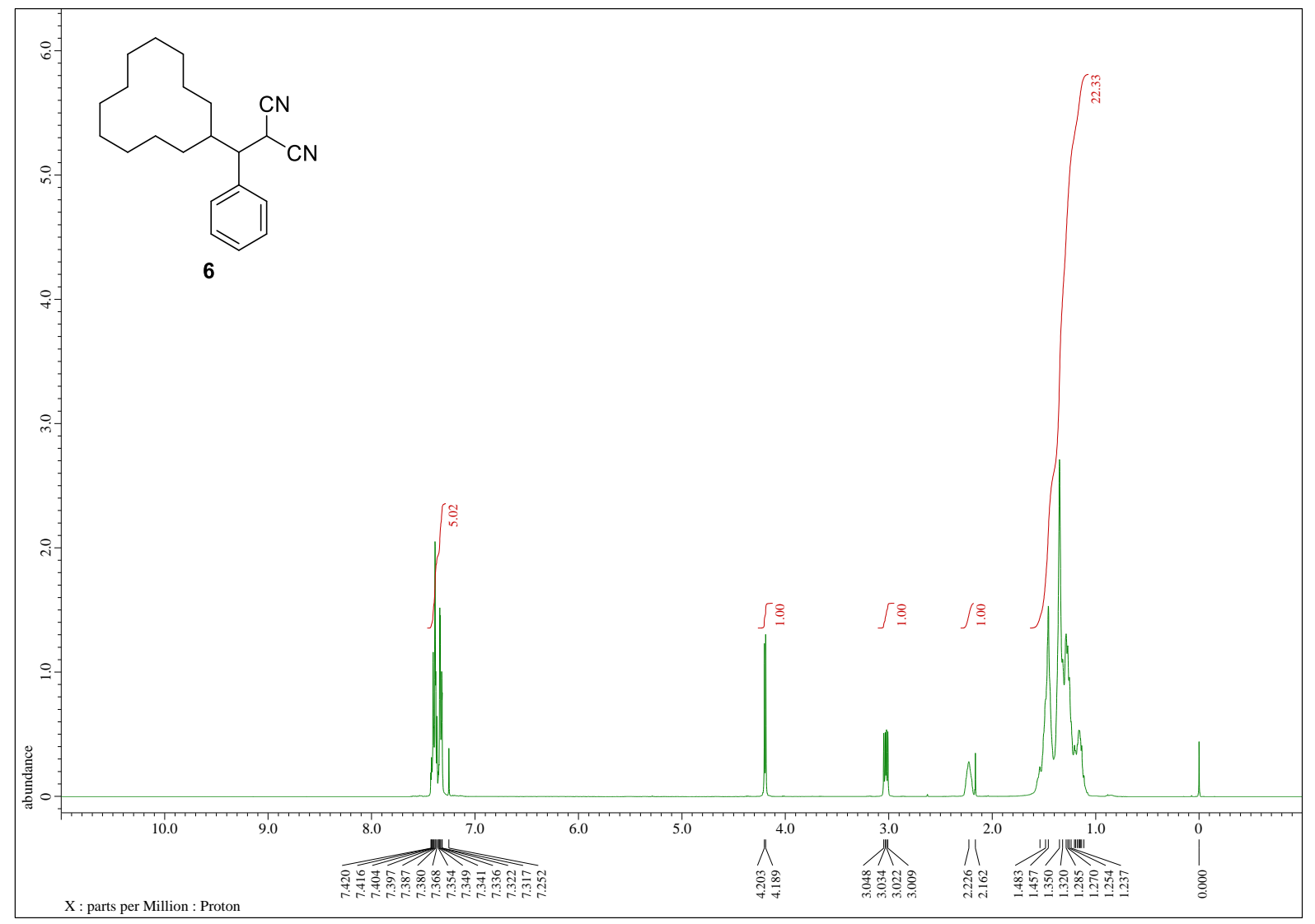

${ }^{13} \mathrm{C}$ NMR spectrum of $6\left(100 \mathrm{MHz}, \mathrm{CDCl}_{3}\right)$

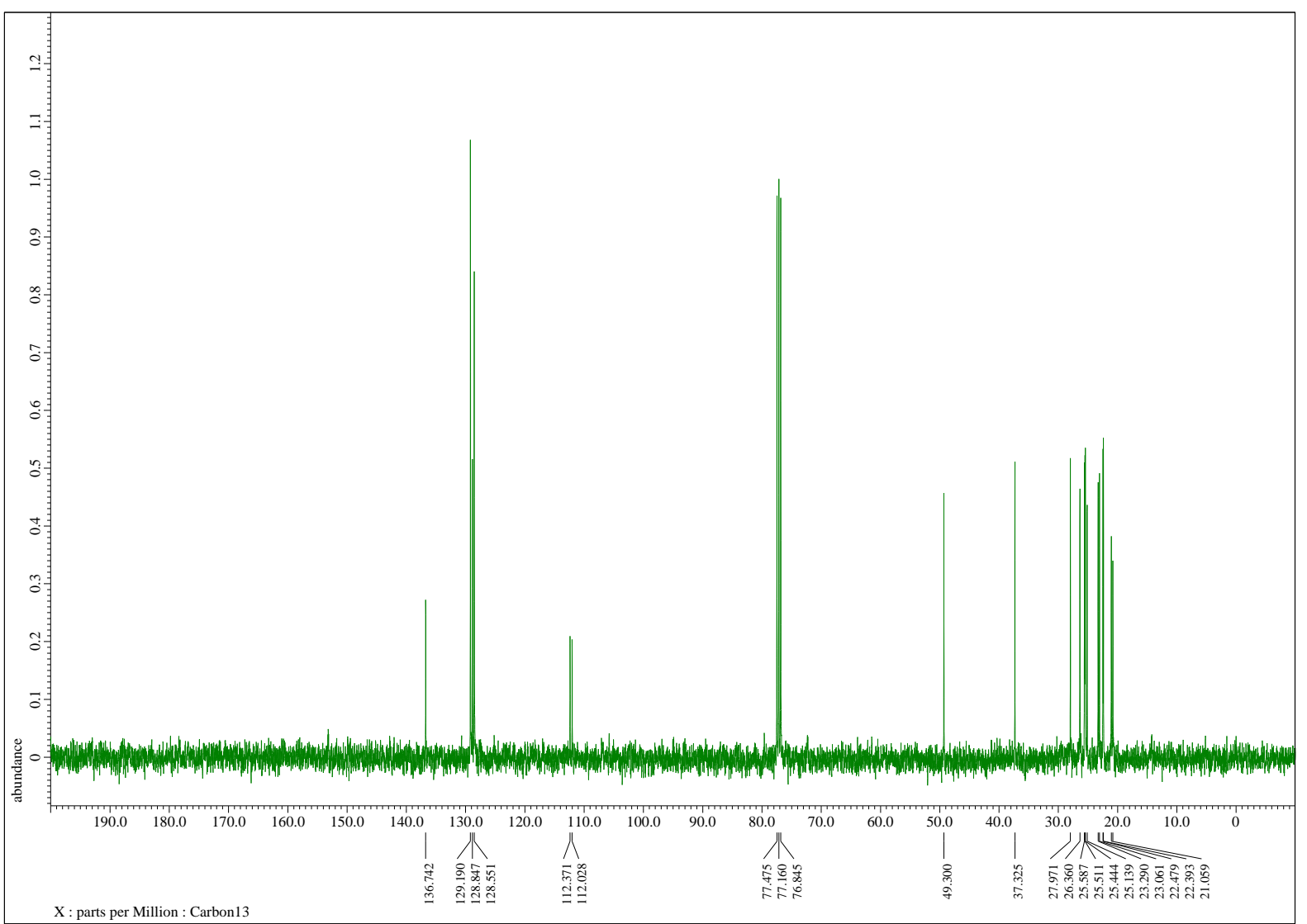


${ }^{1} \mathrm{H}$ NMR spectrum of $7\left(400 \mathrm{MHz}, \mathrm{CDCl}_{3}\right)$

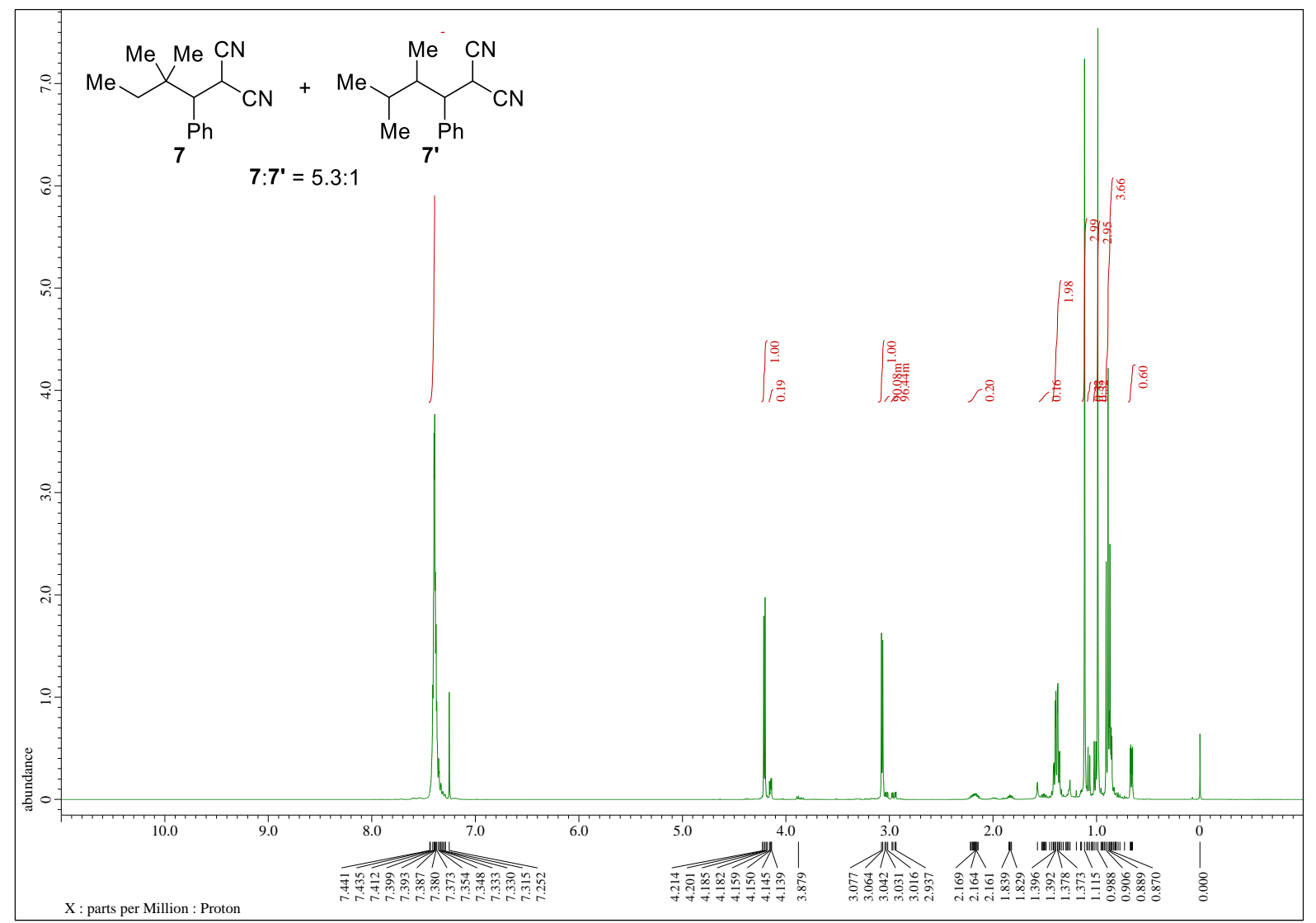

${ }^{13} \mathrm{C}$ NMR spectrum of $7\left(100 \mathrm{MHz}, \mathrm{CDCl}_{3}\right)$

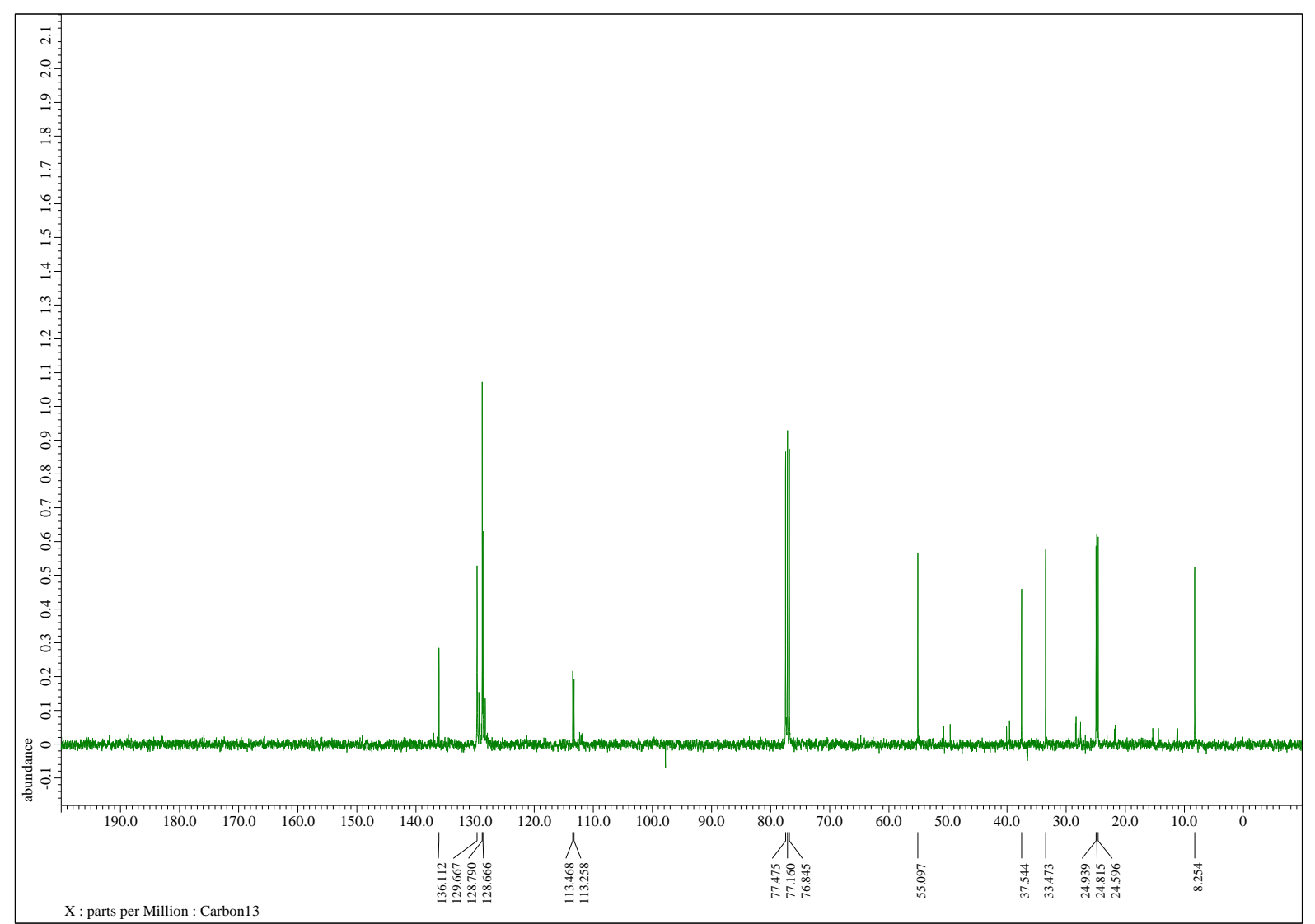


${ }^{1} \mathrm{H} \mathrm{NMR}$ spectrum of $8\left(400 \mathrm{MHz}, \mathrm{CDCl}_{3}\right)$

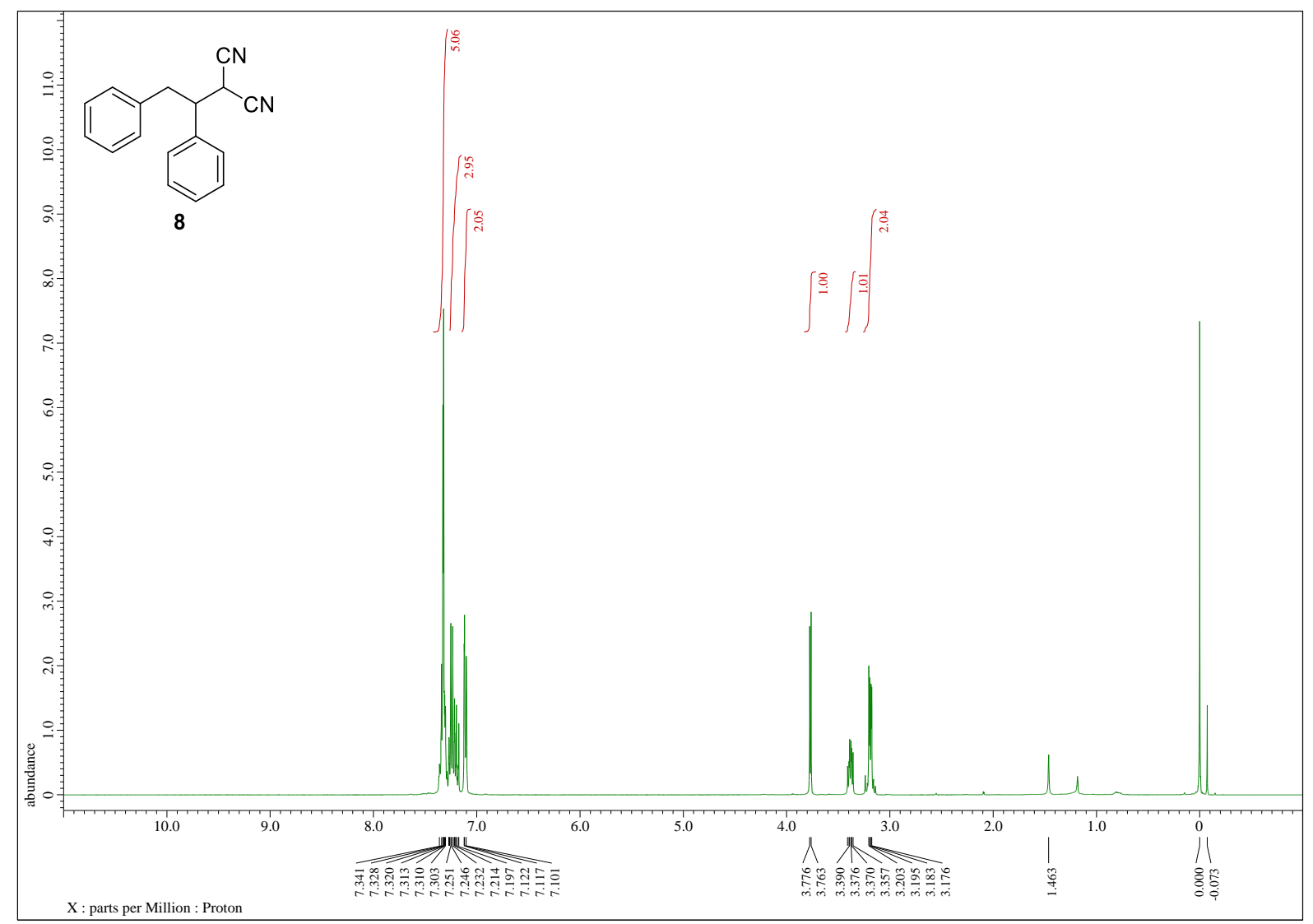

${ }^{13} \mathrm{C}$ NMR spectrum of $\mathbf{8}\left(100 \mathrm{MHz}, \mathrm{CDCl}_{3}\right)$

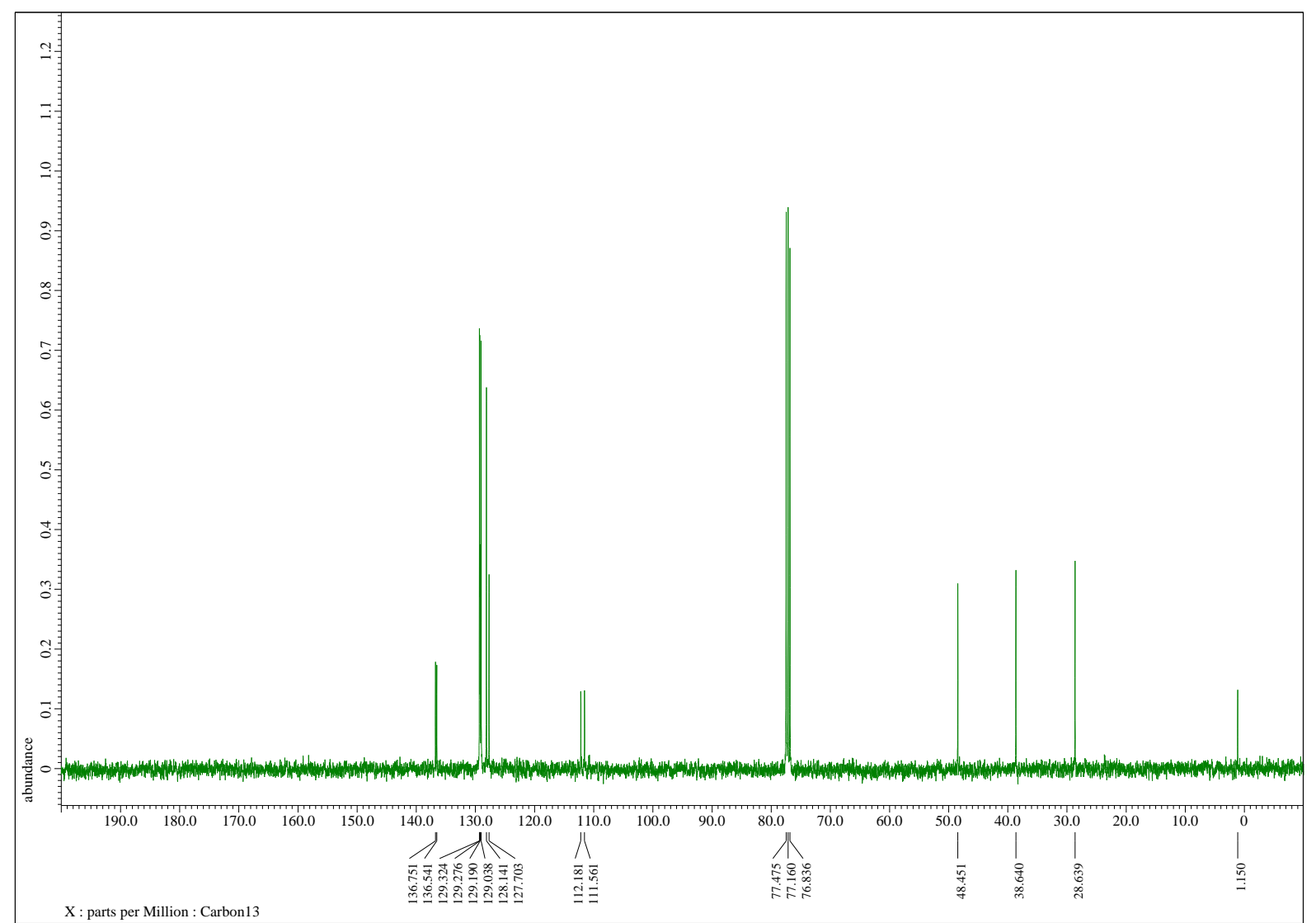


${ }^{1} \mathrm{H}$ NMR spectrum of 9' (400 MHz, $\mathrm{CDCl}_{3}$ )

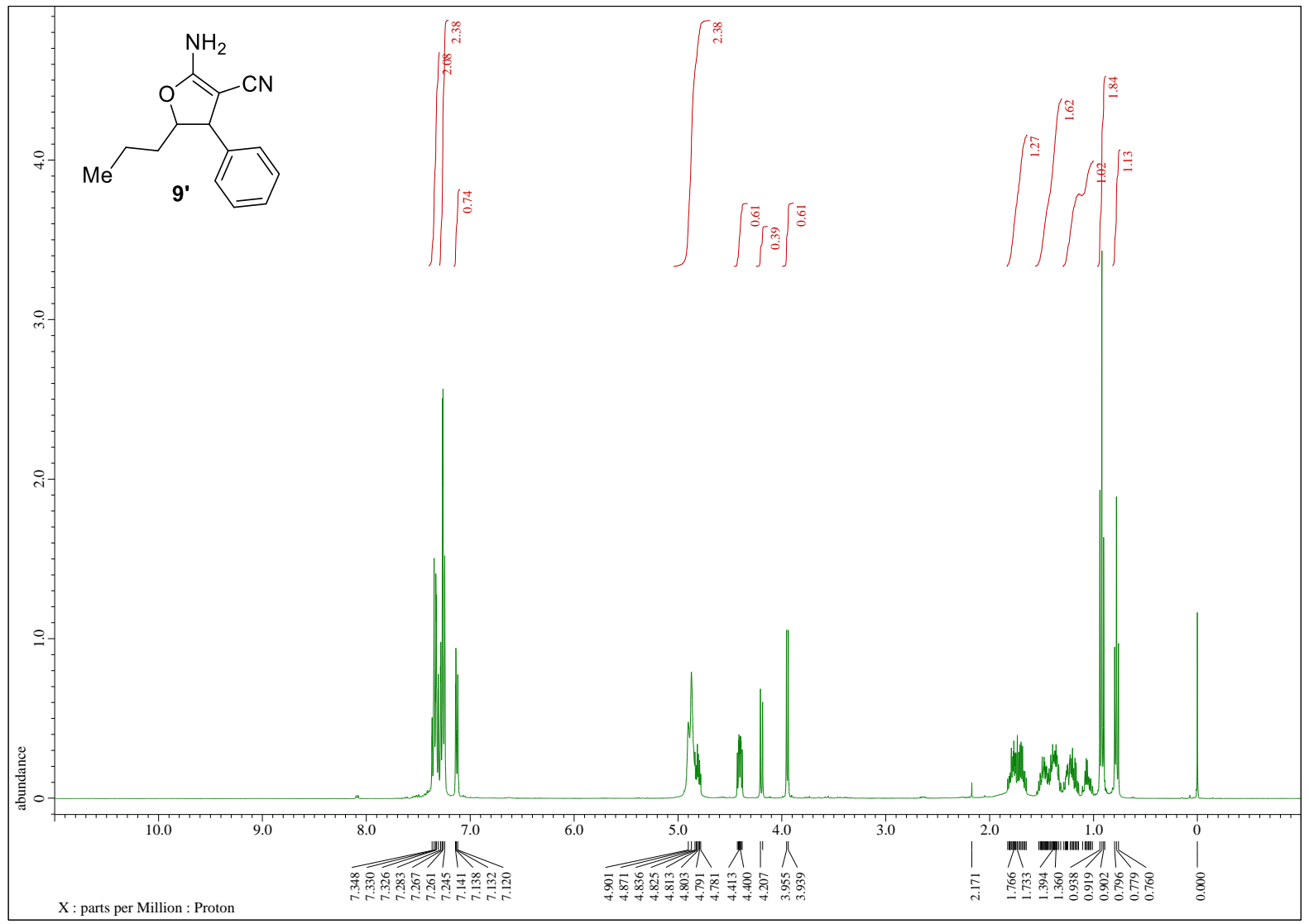

${ }^{13} \mathrm{C}$ NMR spectrum of 9' (100 MHz, $\left.\mathrm{CDCl}_{3}\right)$

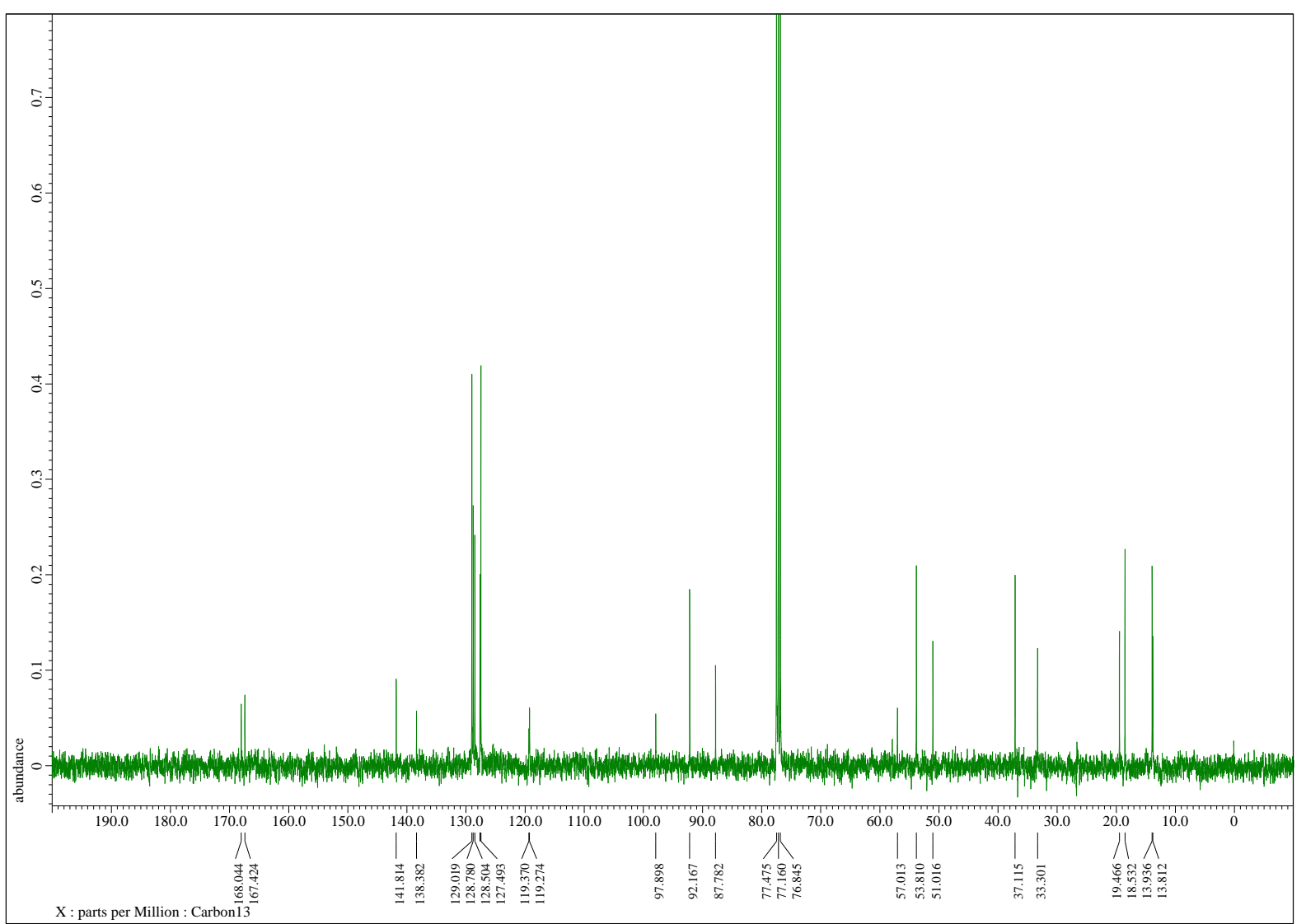


${ }^{1} \mathrm{H}$ NMR spectrum of $\mathbf{1 0}$ ' $\left(400 \mathrm{MHz}, \mathrm{CDCl}_{3}\right)$

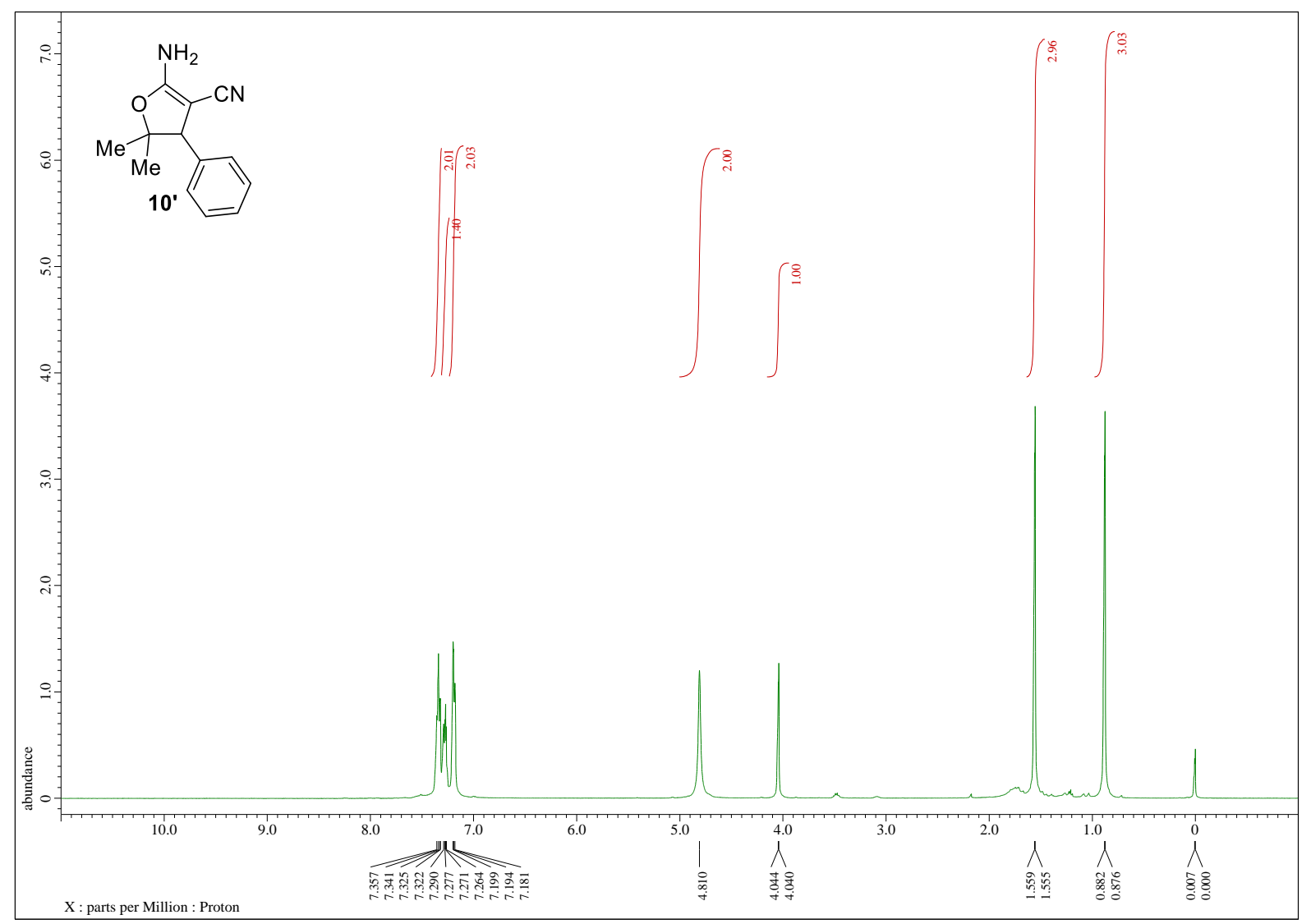

${ }^{13} \mathrm{C}$ NMR spectrum of $\mathbf{1 0}$ ' (100 MHz, $\left.\mathrm{CDCl}_{3}\right)$

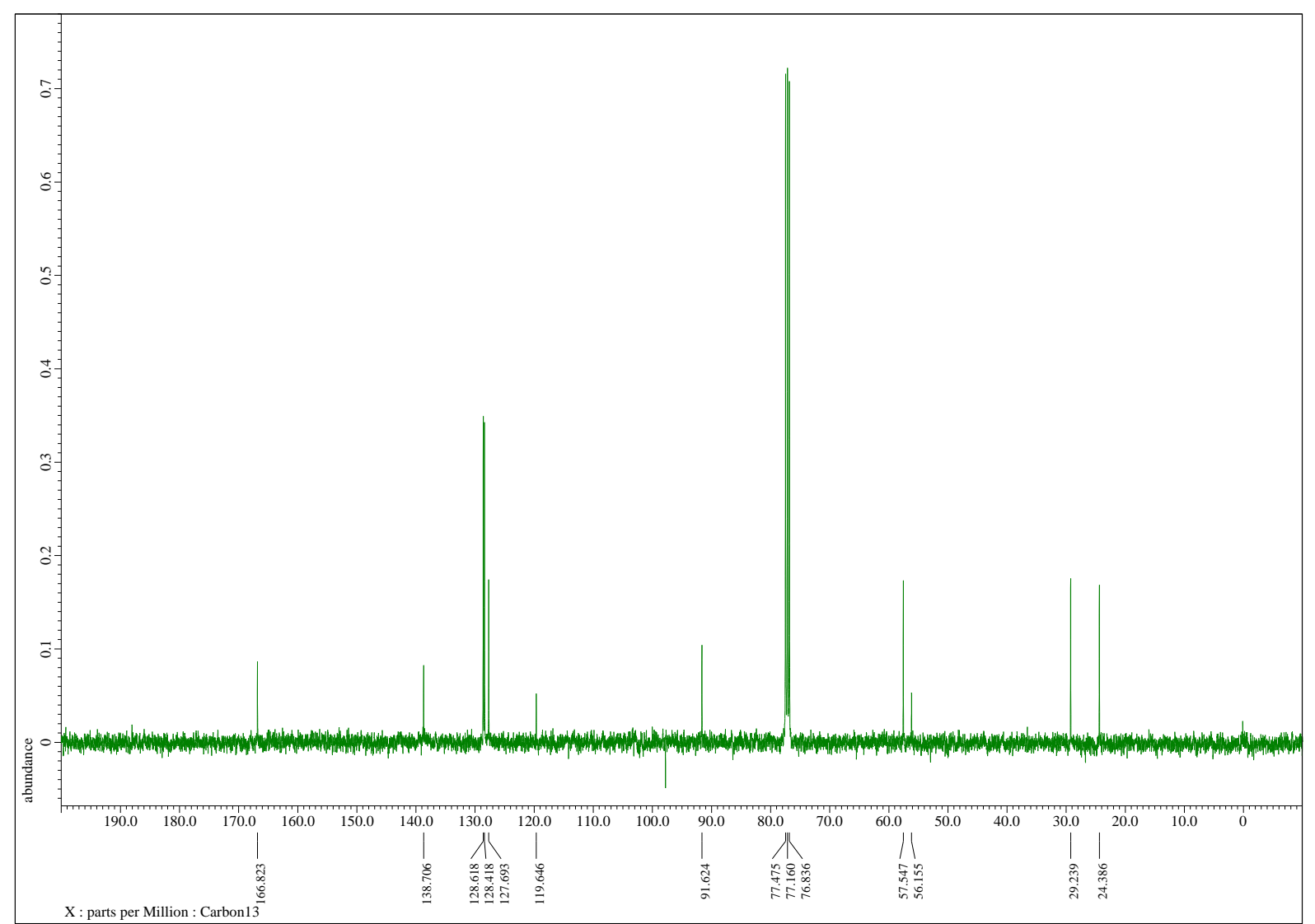


${ }^{1} \mathrm{H}$ NMR spectrum of $11\left(400 \mathrm{MHz}, \mathrm{CDCl}_{3}\right)$

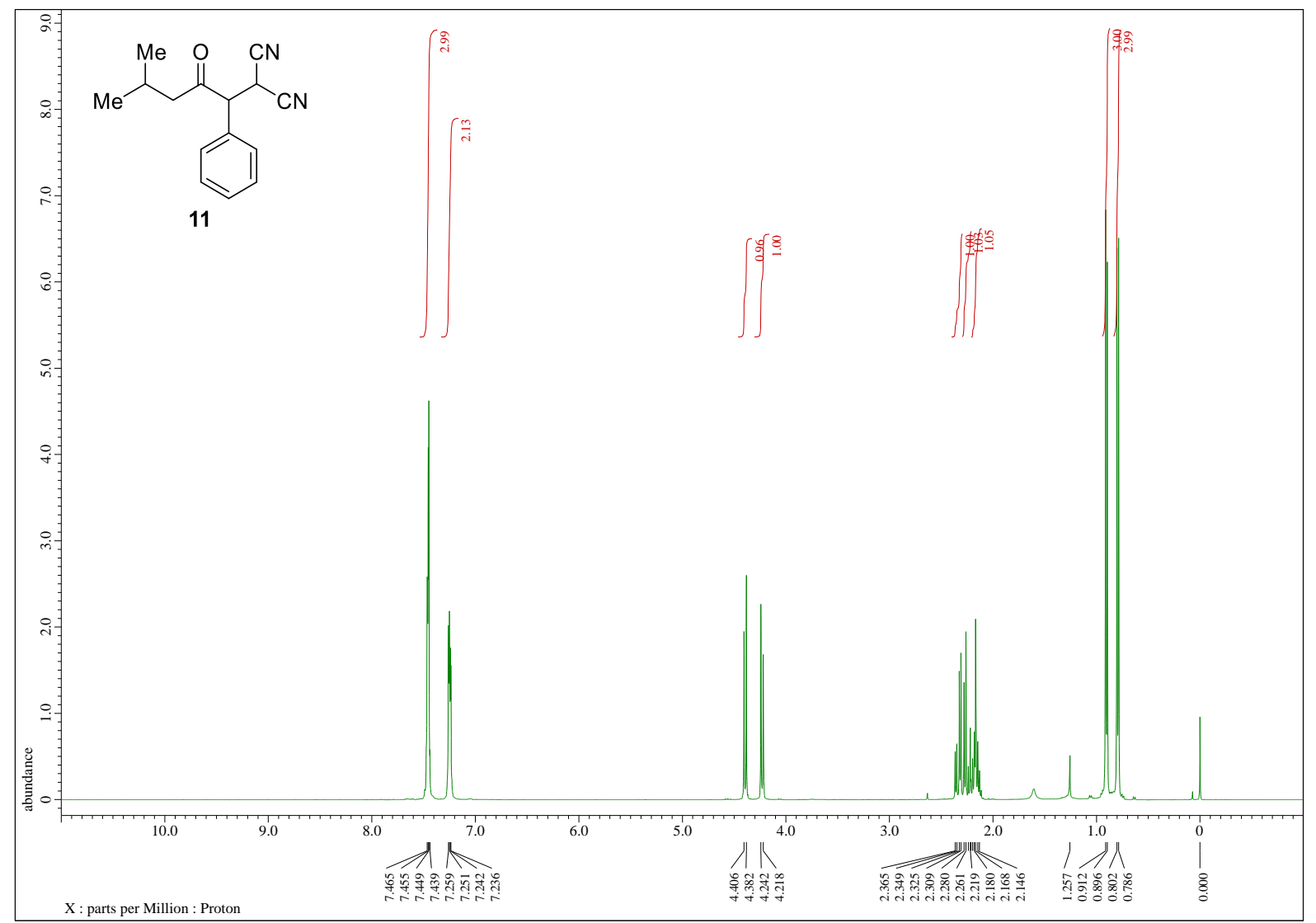

${ }^{13} \mathrm{C}$ NMR spectrum of $\mathbf{1 1}\left(100 \mathrm{MHz}, \mathrm{CDCl}_{3}\right)$

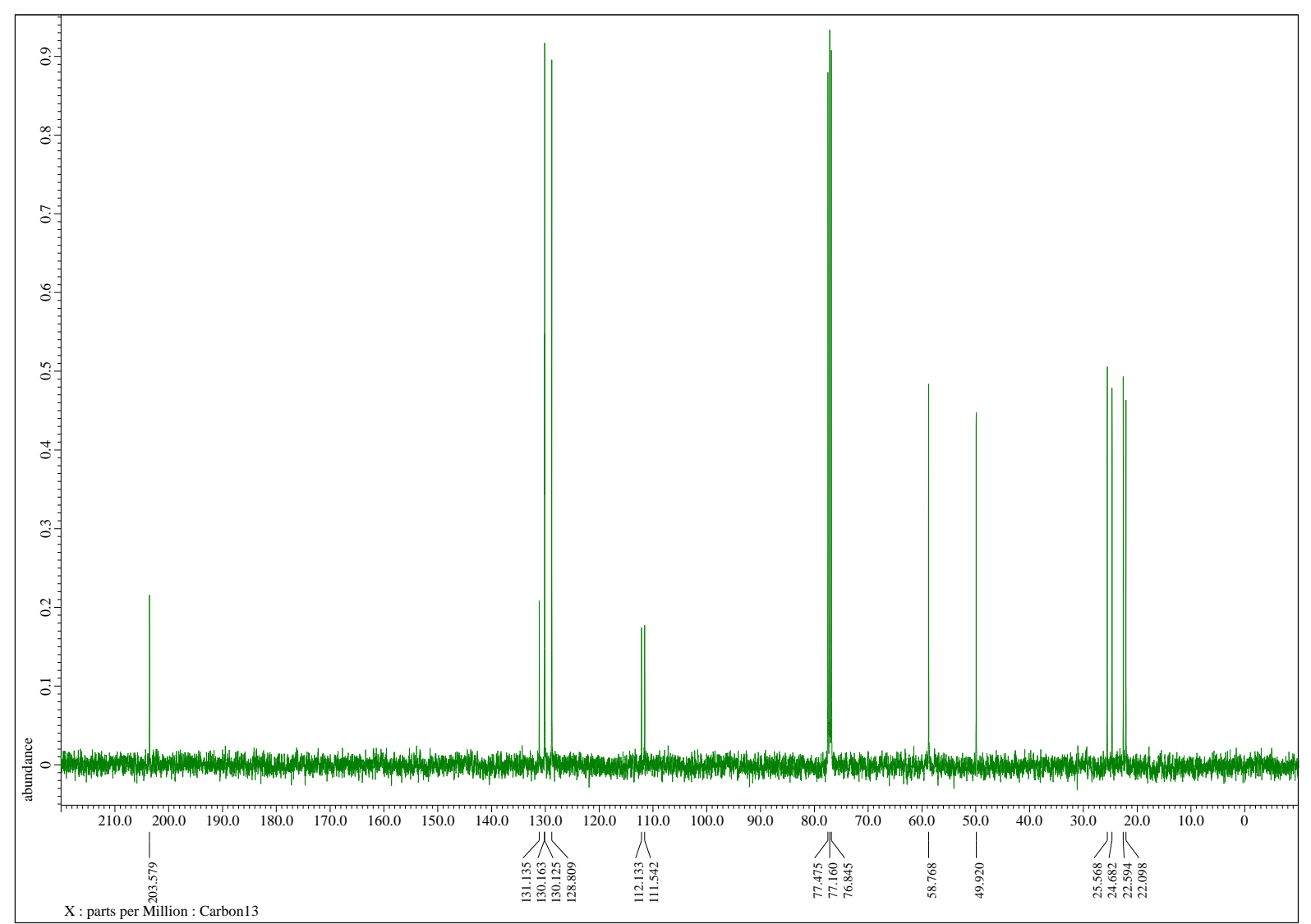


${ }^{1} \mathrm{H}$ NMR spectrum of $12\left(400 \mathrm{MHz}, \mathrm{CDCl}_{3}\right)$

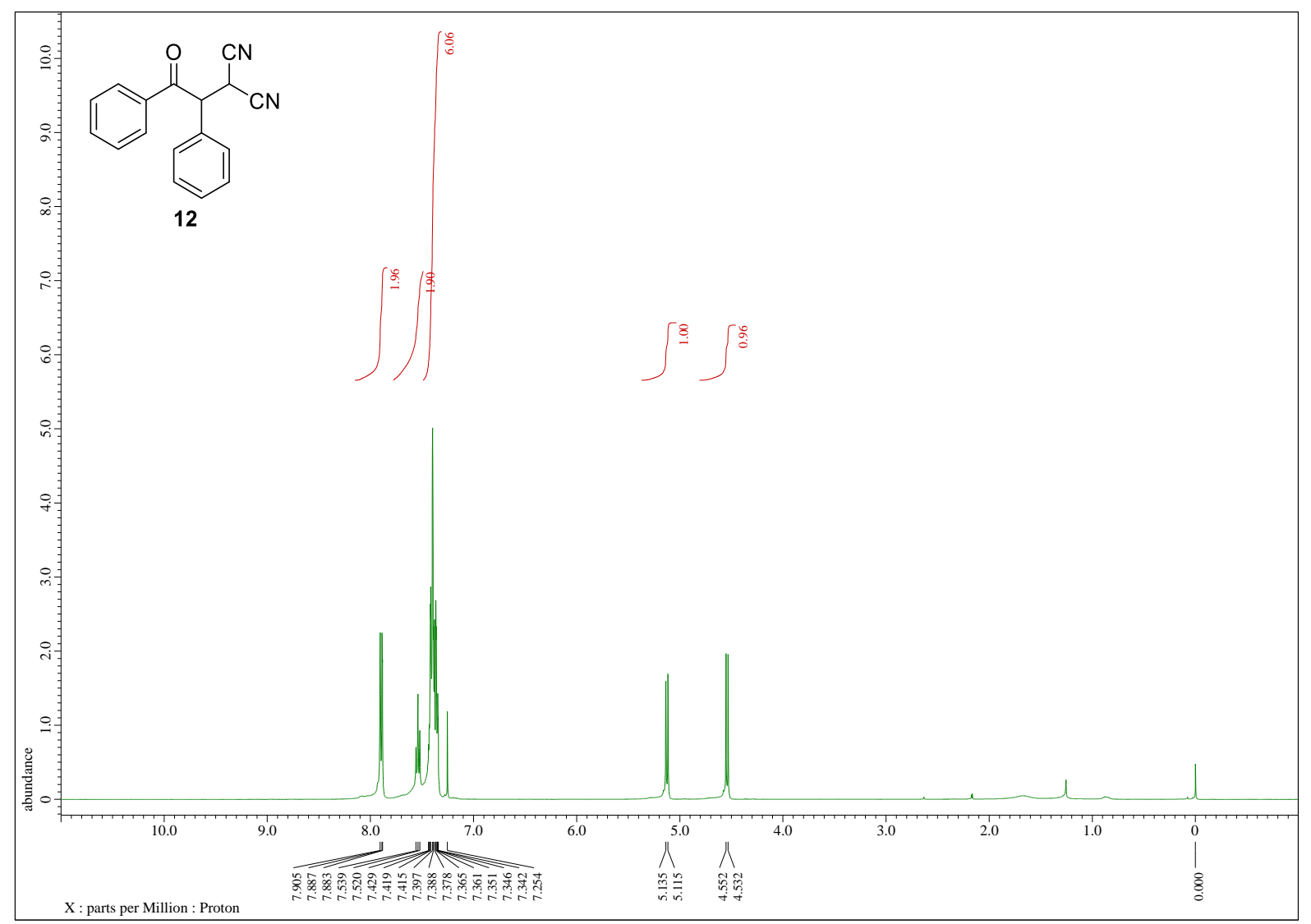

${ }^{13} \mathrm{C}$ NMR spectrum of $\mathbf{1 2}\left(100 \mathrm{MHz}, \mathrm{CDCl}_{3}\right)$

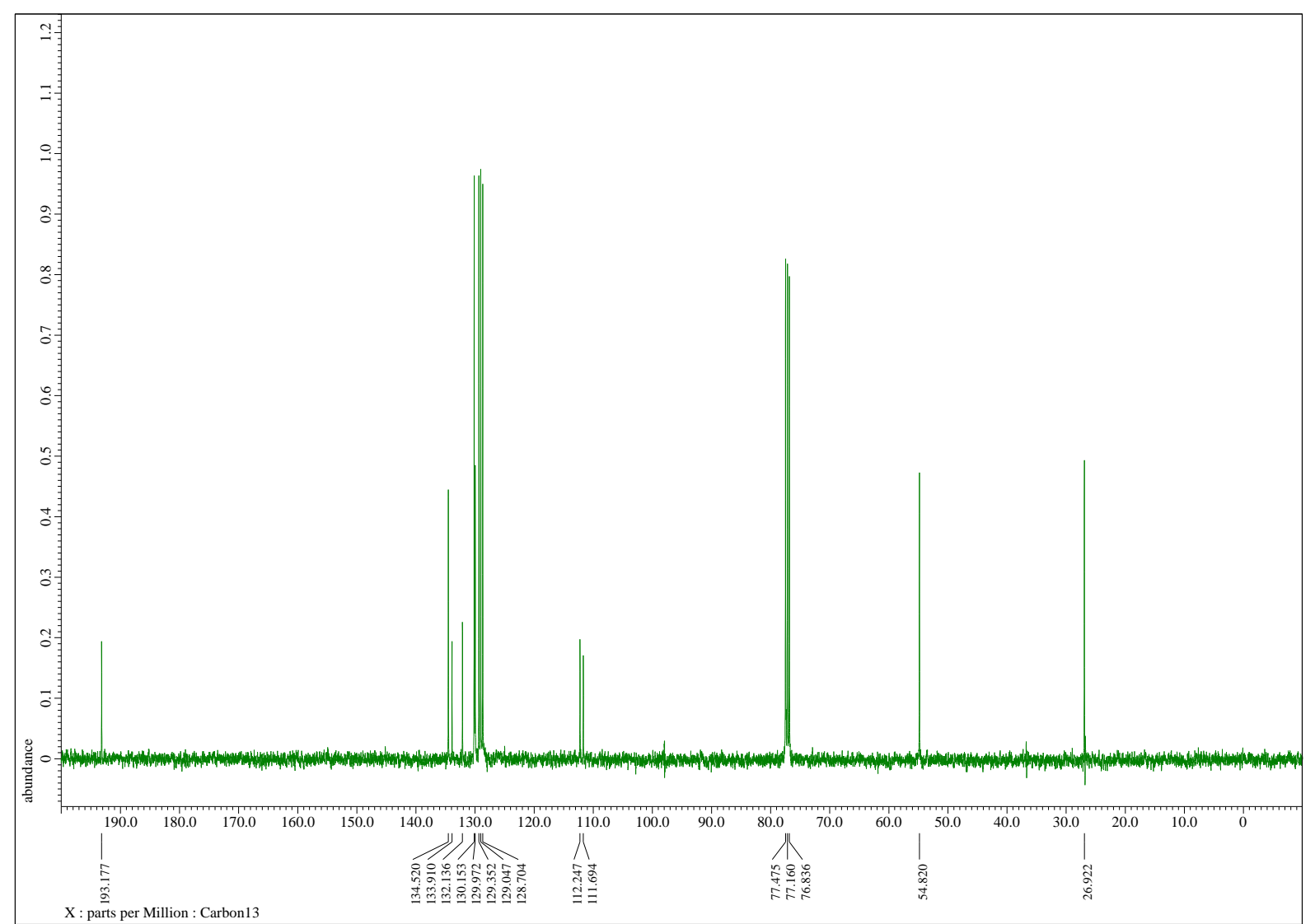


${ }^{1} \mathrm{H}$ NMR spectrum of $13\left(400 \mathrm{MHz}, \mathrm{CDCl}_{3}\right)$

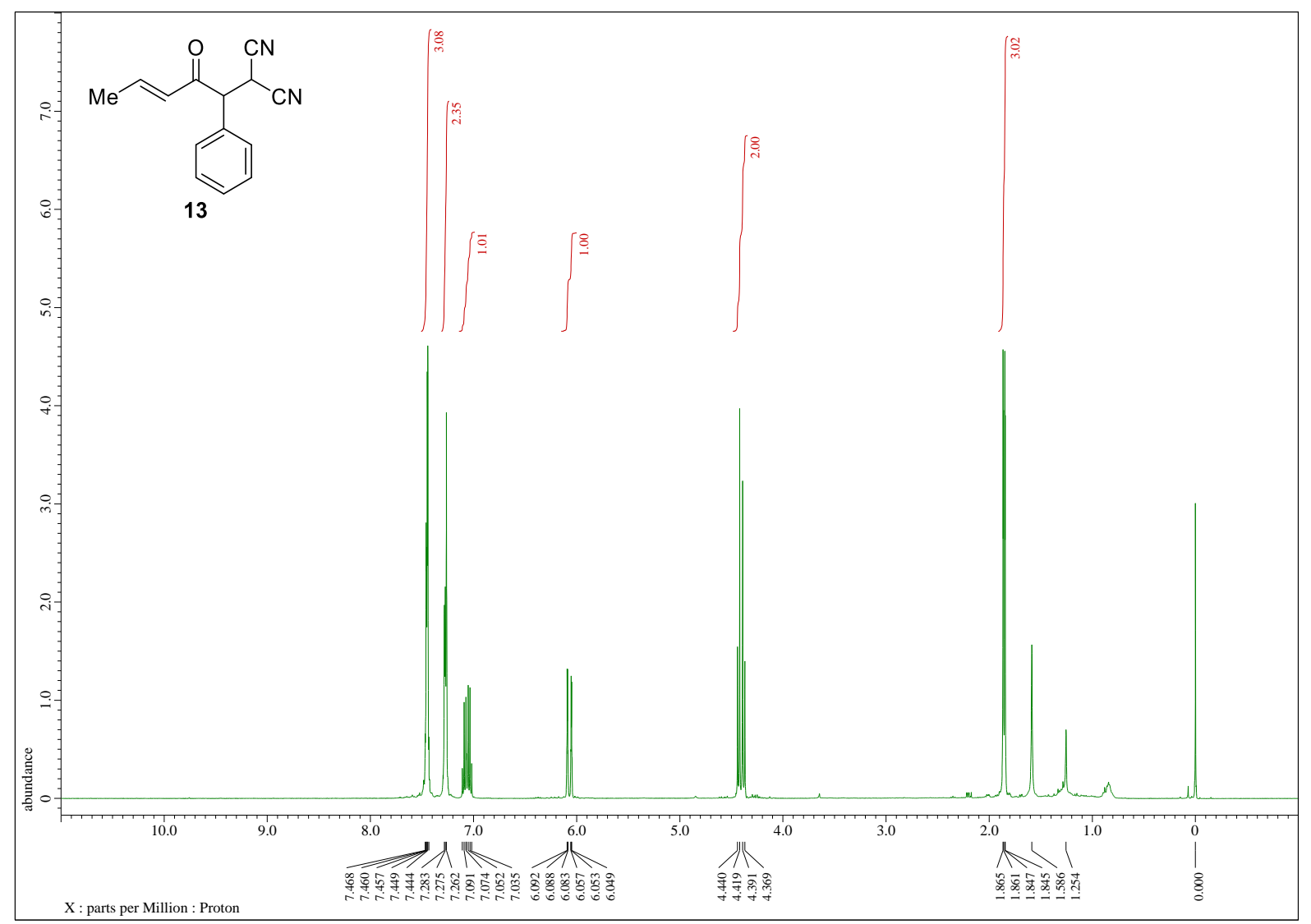

${ }^{13} \mathrm{C}$ NMR spectrum of $\mathbf{1 3}\left(100 \mathrm{MHz}, \mathrm{CDCl}_{3}\right)$

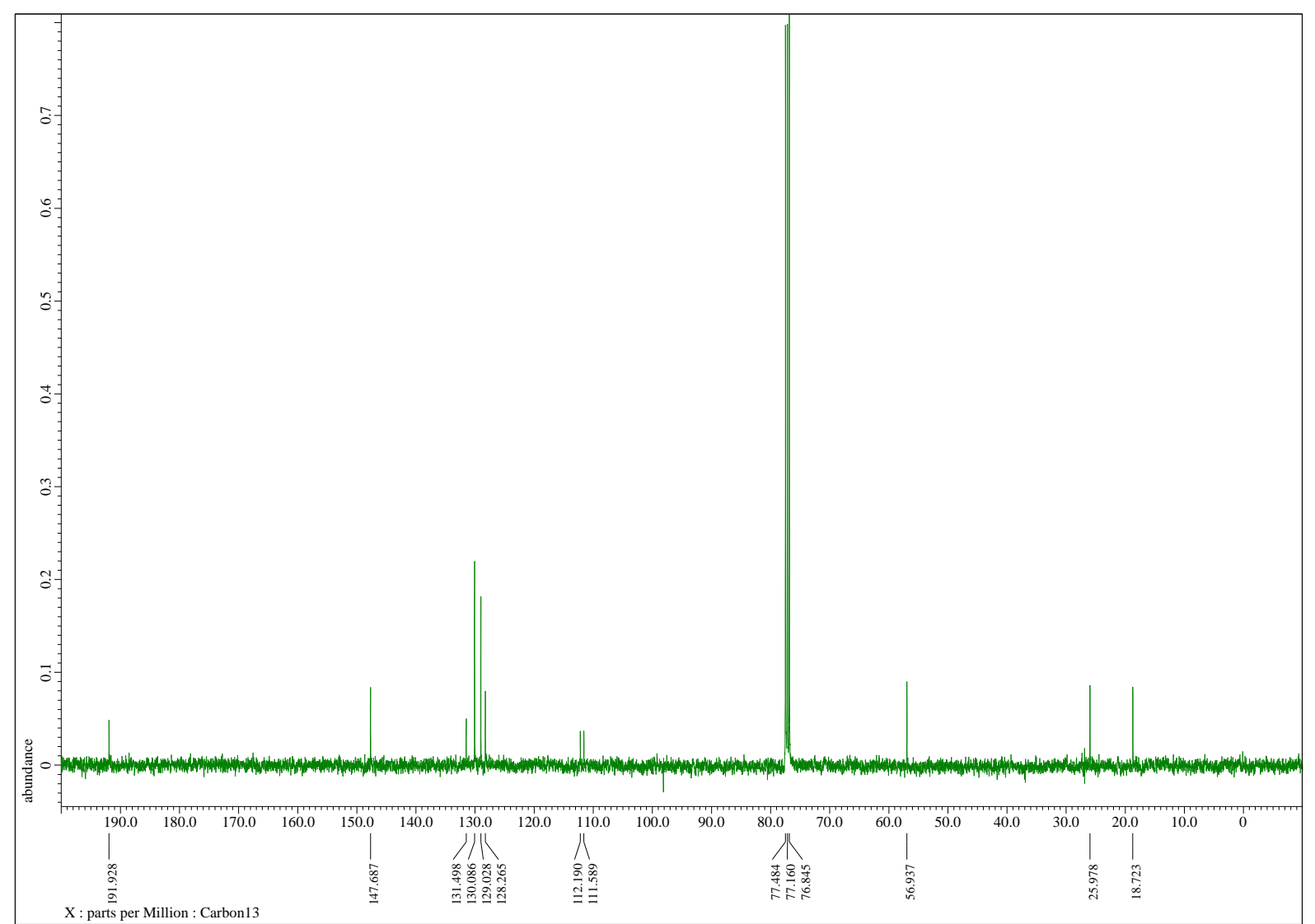


${ }^{1} \mathrm{H}$ NMR spectrum of $14\left(400 \mathrm{MHz}, \mathrm{CDCl}_{3}\right)$

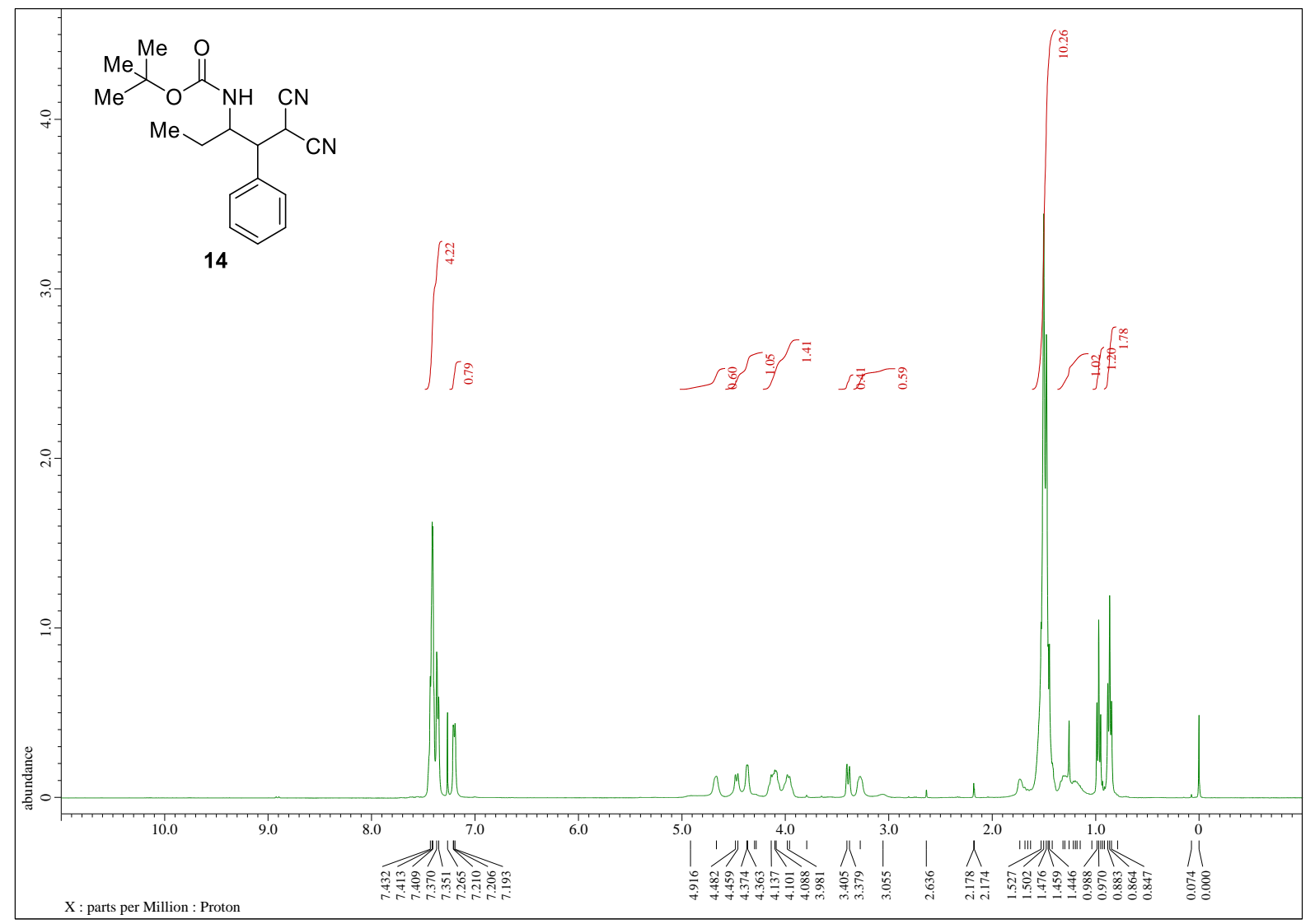

${ }^{13} \mathrm{C}$ NMR spectrum of $\mathbf{1 4}\left(100 \mathrm{MHz}, \mathrm{CDCl}_{3}\right)$

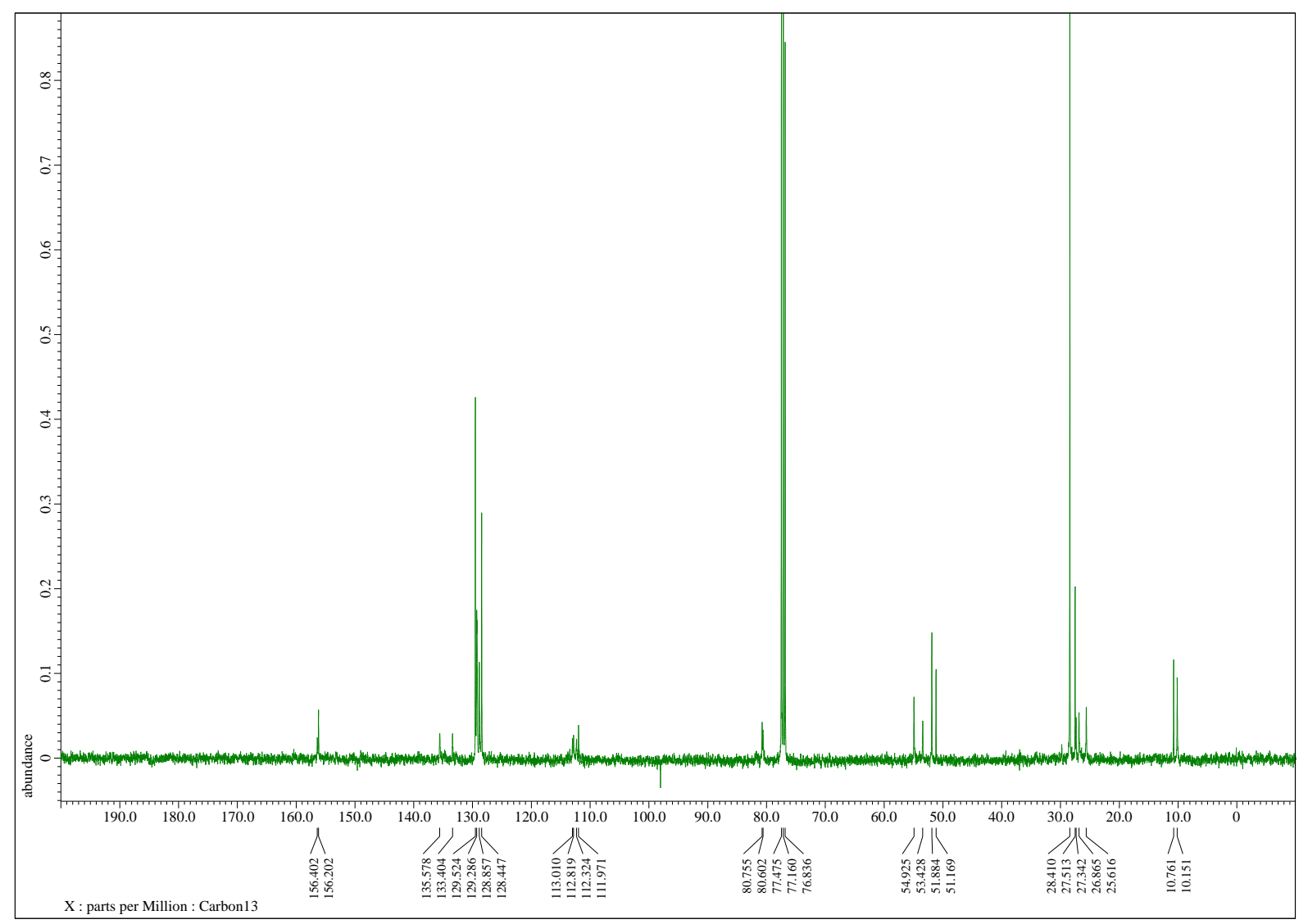


${ }^{1} \mathrm{H}$ NMR spectrum of 15 (400 MHz, $\left.\mathrm{CDCl}_{3}\right)$

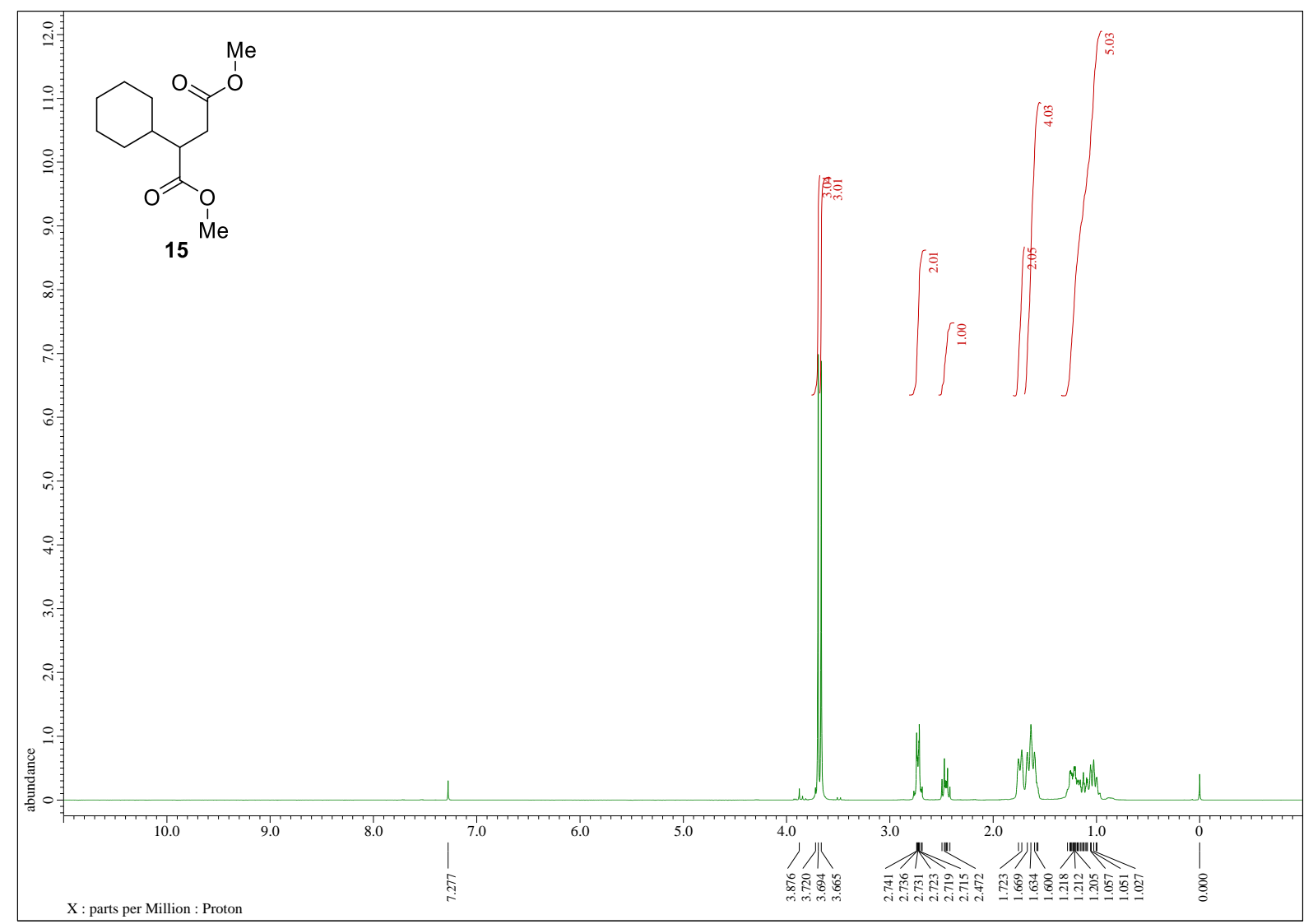

${ }^{13} \mathrm{C}$ NMR spectrum of $\mathbf{1 5}\left(100 \mathrm{MHz}, \mathrm{CDCl}_{3}\right)$

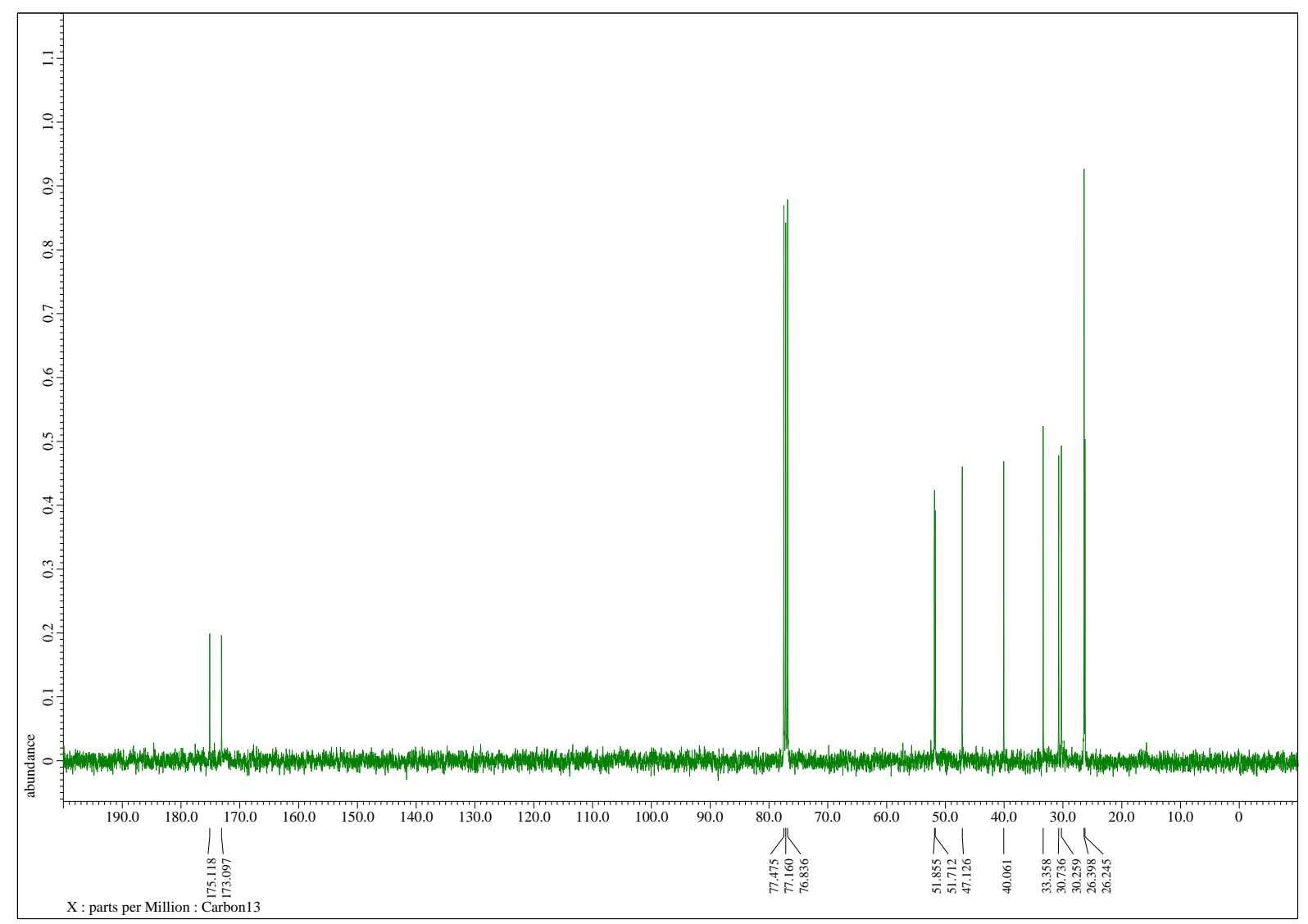


${ }^{1} \mathrm{H}$ NMR spectrum of $\mathbf{1 6}\left(400 \mathrm{MHz}, \mathrm{CDCl}_{3}\right)$

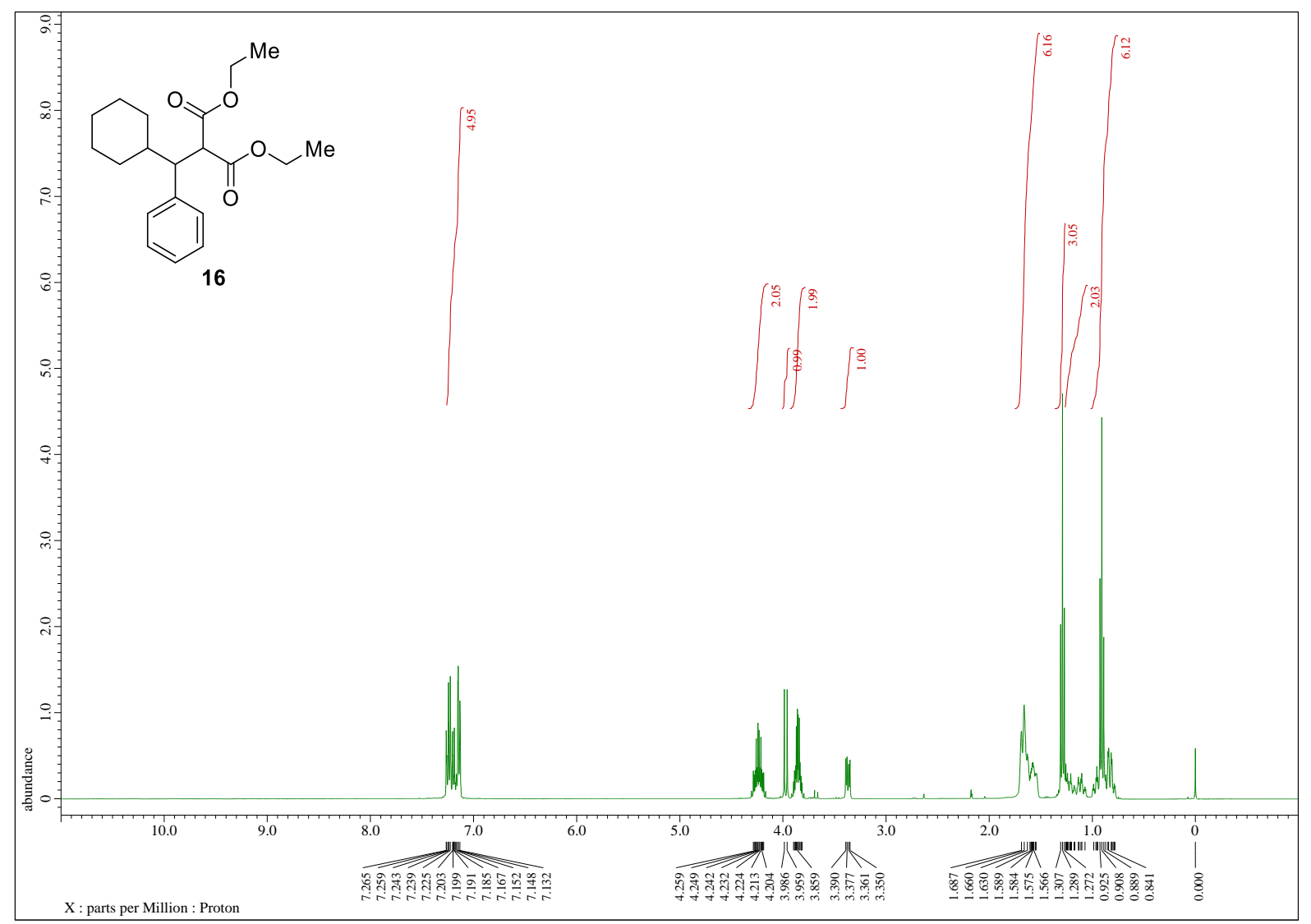

${ }^{13} \mathrm{C}$ NMR spectrum of $\mathbf{1 6}\left(100 \mathrm{MHz}, \mathrm{CDCl}_{3}\right)$

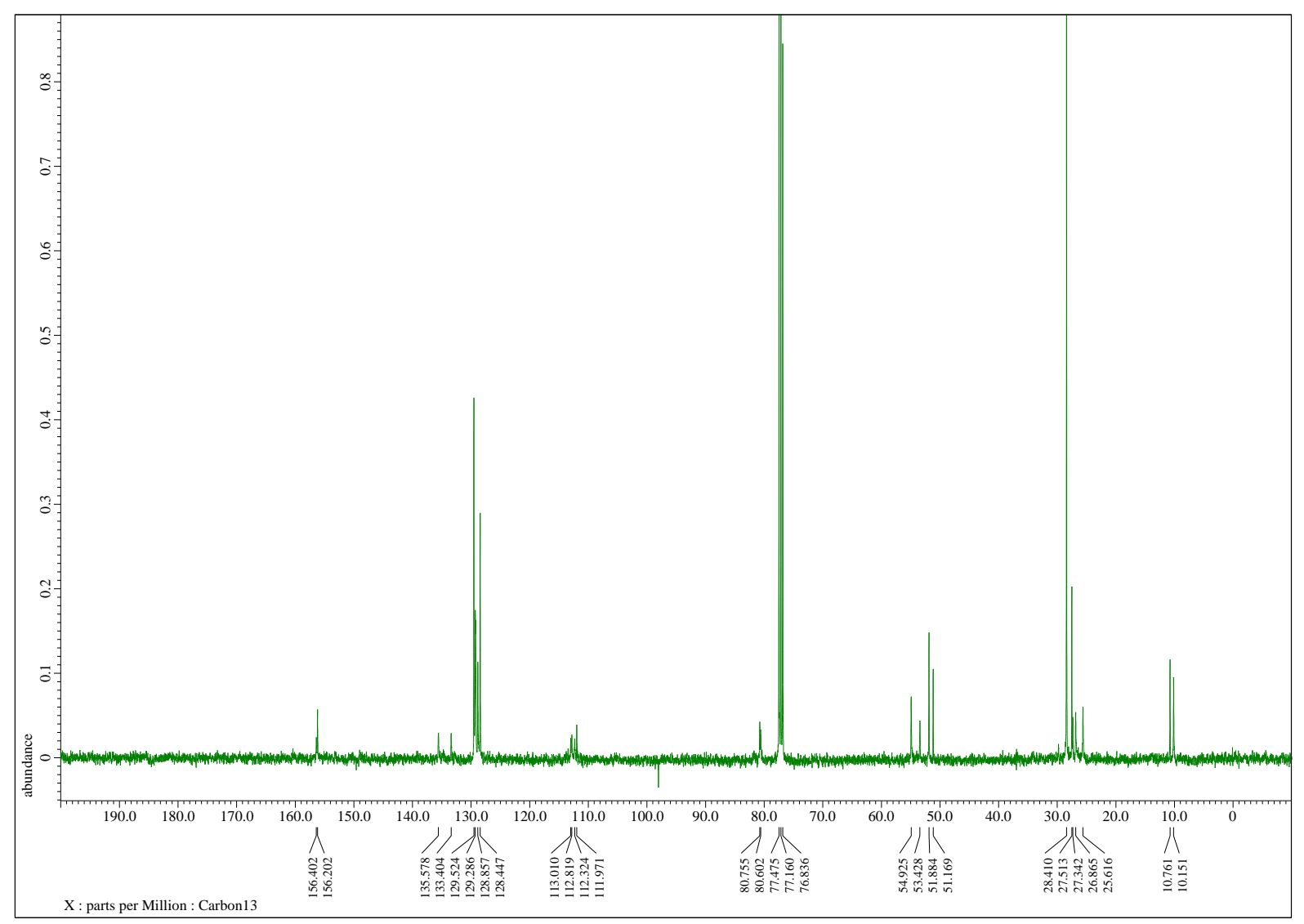


${ }^{1} \mathrm{H}$ NMR spectrum of $17\left(400 \mathrm{MHz}, \mathrm{CDCl}_{3}\right)$

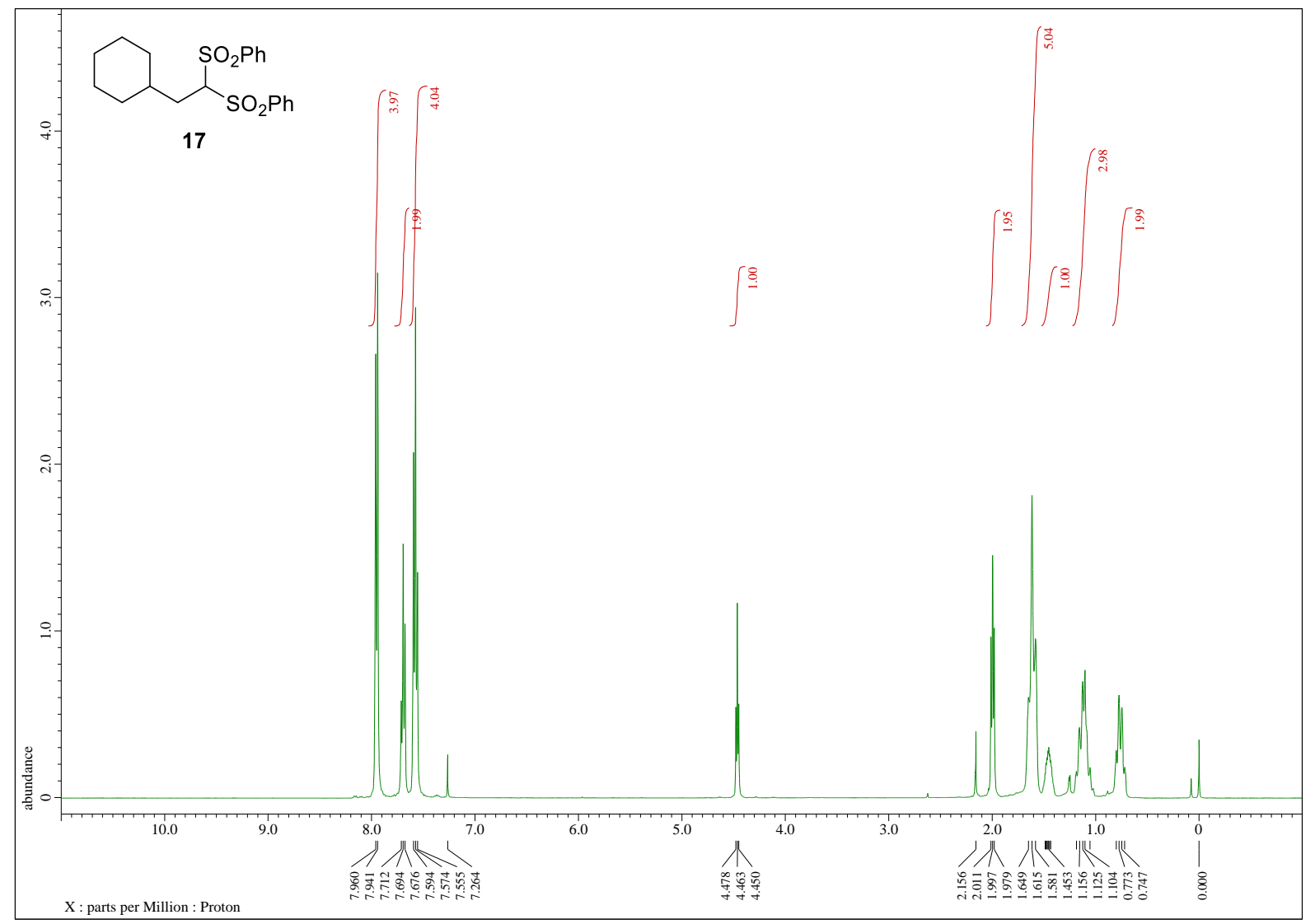

${ }^{13} \mathrm{C}$ NMR spectrum of $\mathbf{1 7}\left(100 \mathrm{MHz}, \mathrm{CDCl}_{3}\right)$

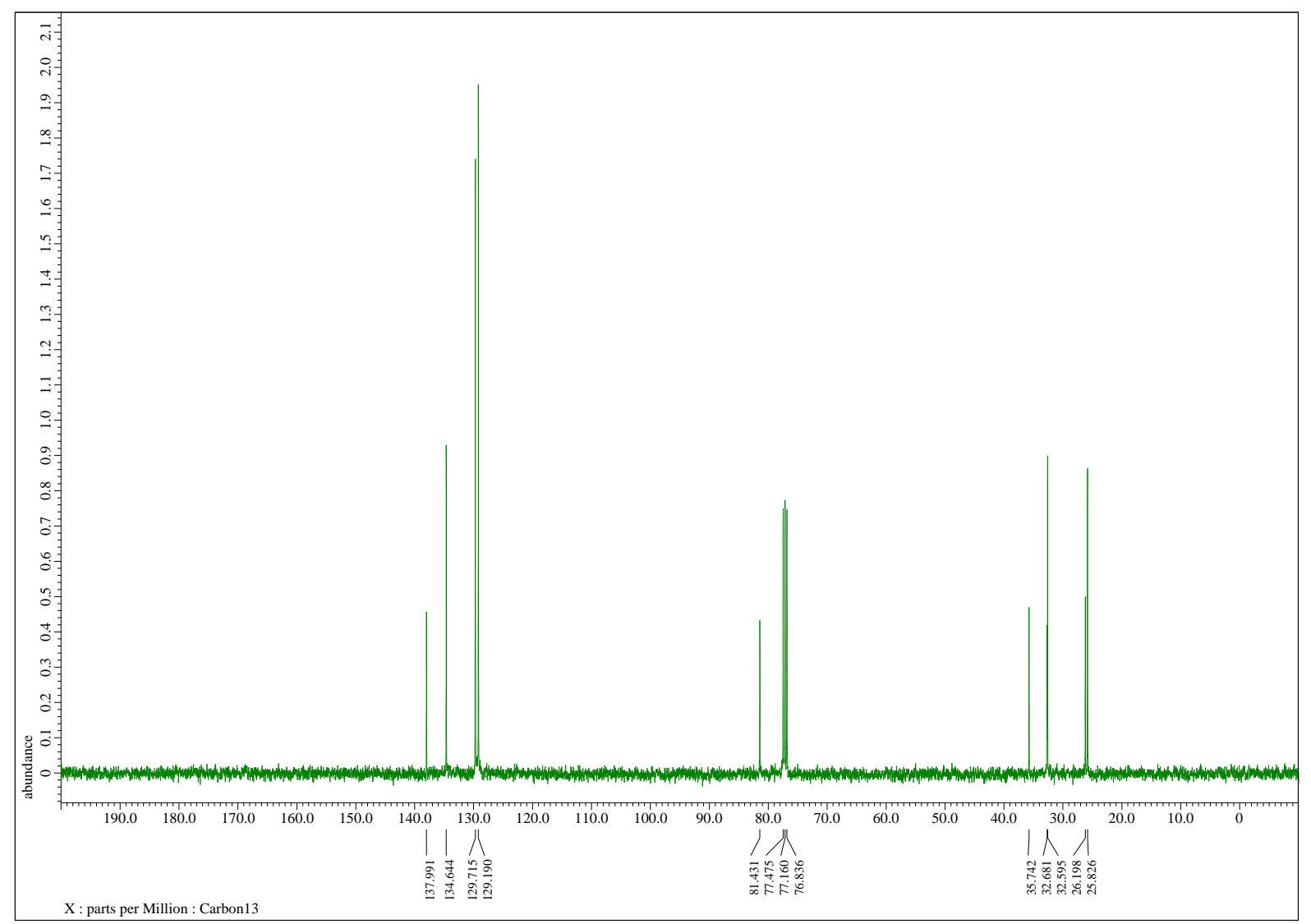


${ }^{1} \mathrm{H}$ NMR spectrum of $18\left(400 \mathrm{MHz}, \mathrm{CDCl}_{3}\right)$

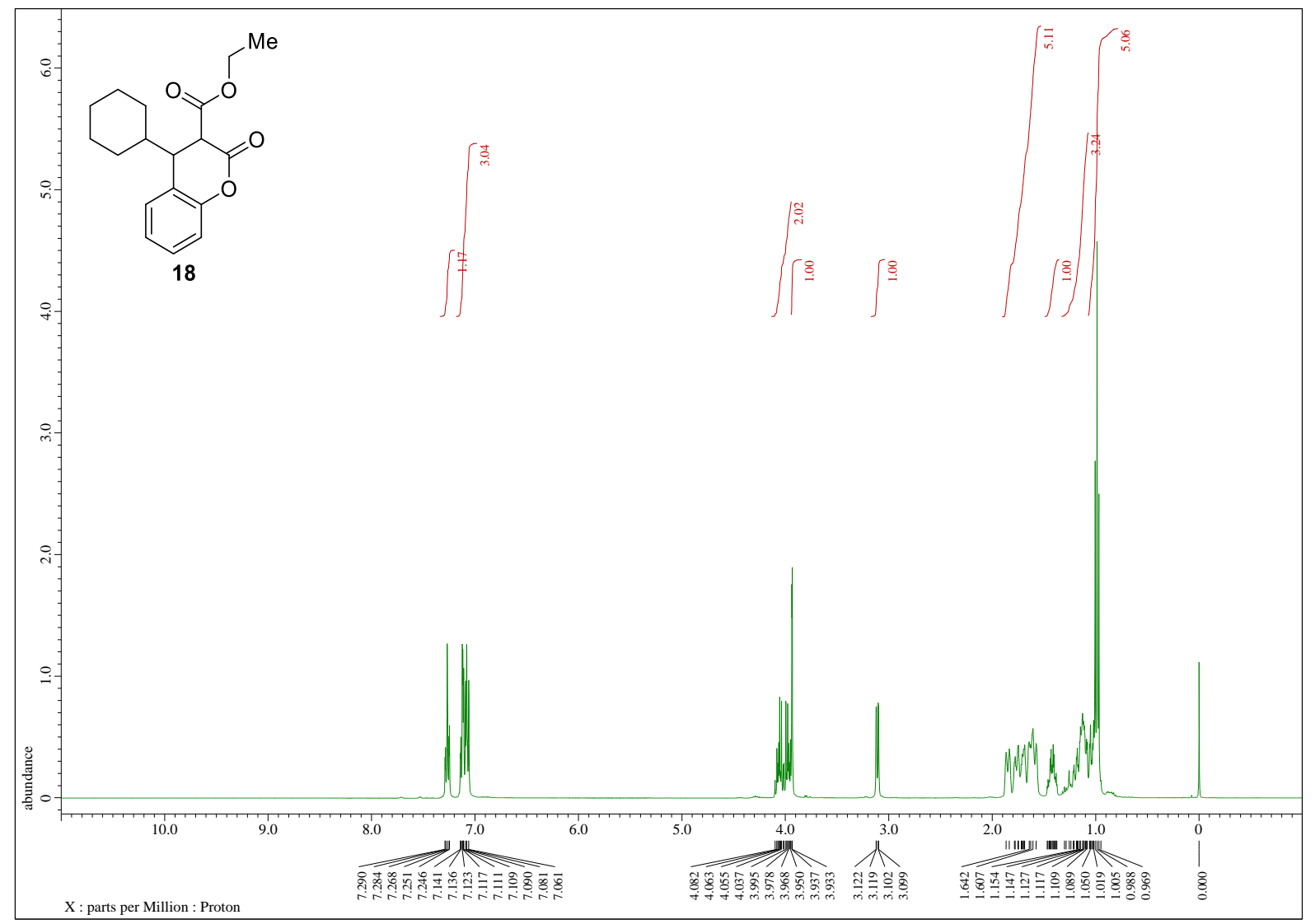

${ }^{13} \mathrm{C}$ NMR spectrum of $\mathbf{1 8}\left(100 \mathrm{MHz}, \mathrm{CDCl}_{3}\right)$

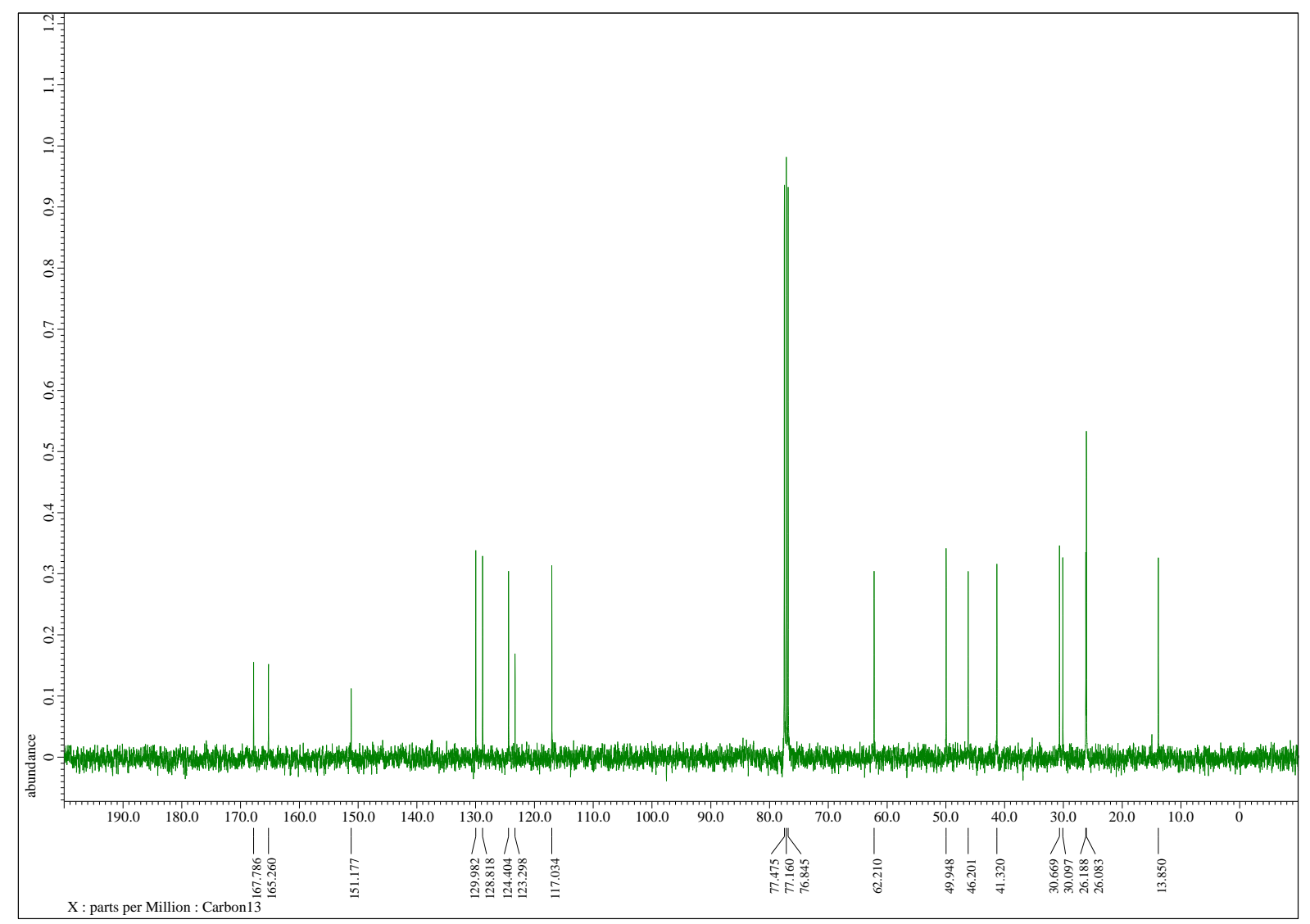


${ }^{1} \mathrm{H}$ NMR spectrum of $20\left(400 \mathrm{MHz}, \mathrm{CDCl}_{3}\right)$

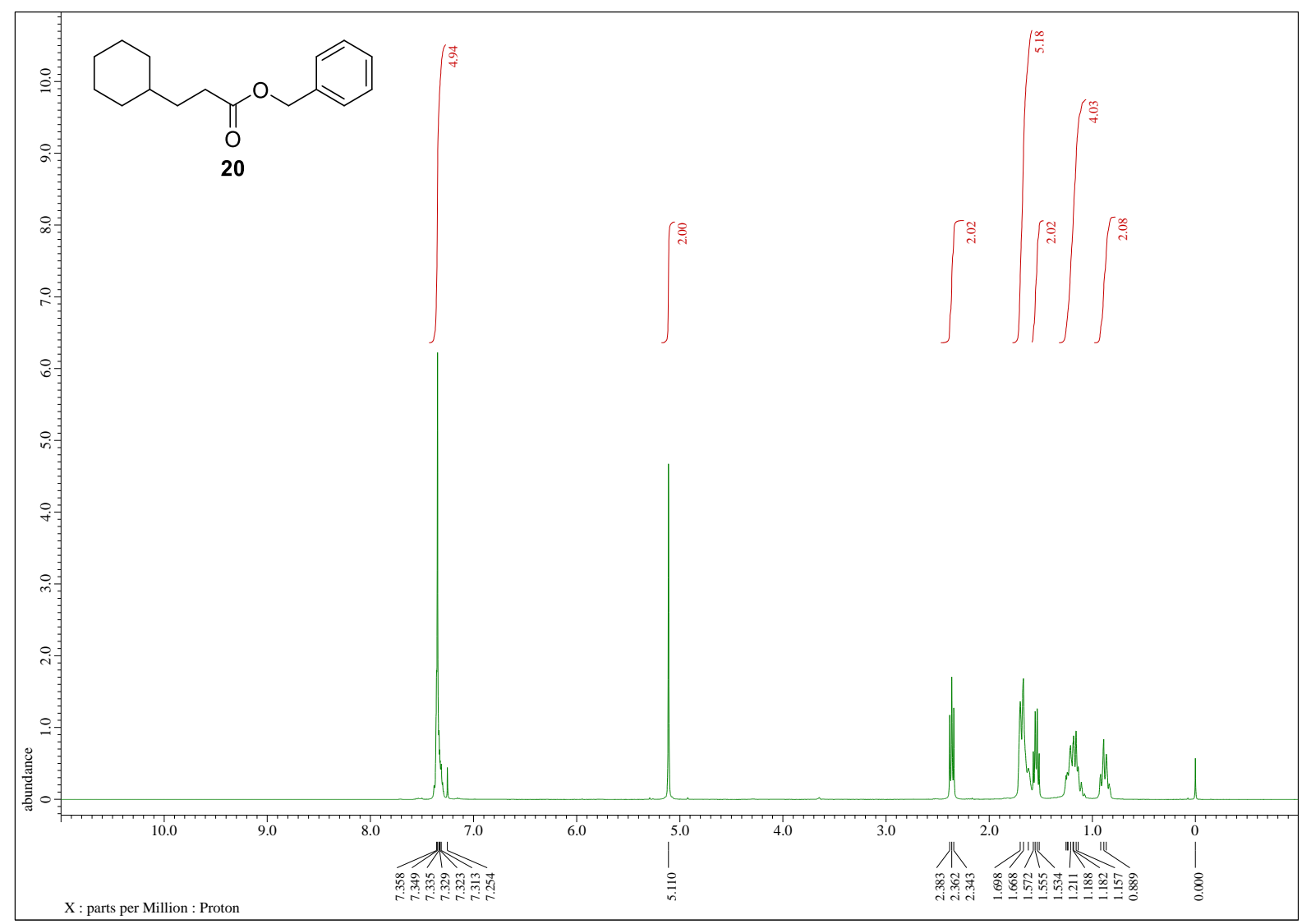

${ }^{13} \mathrm{C}$ NMR spectrum of $20\left(100 \mathrm{MHz}, \mathrm{CDCl}_{3}\right)$

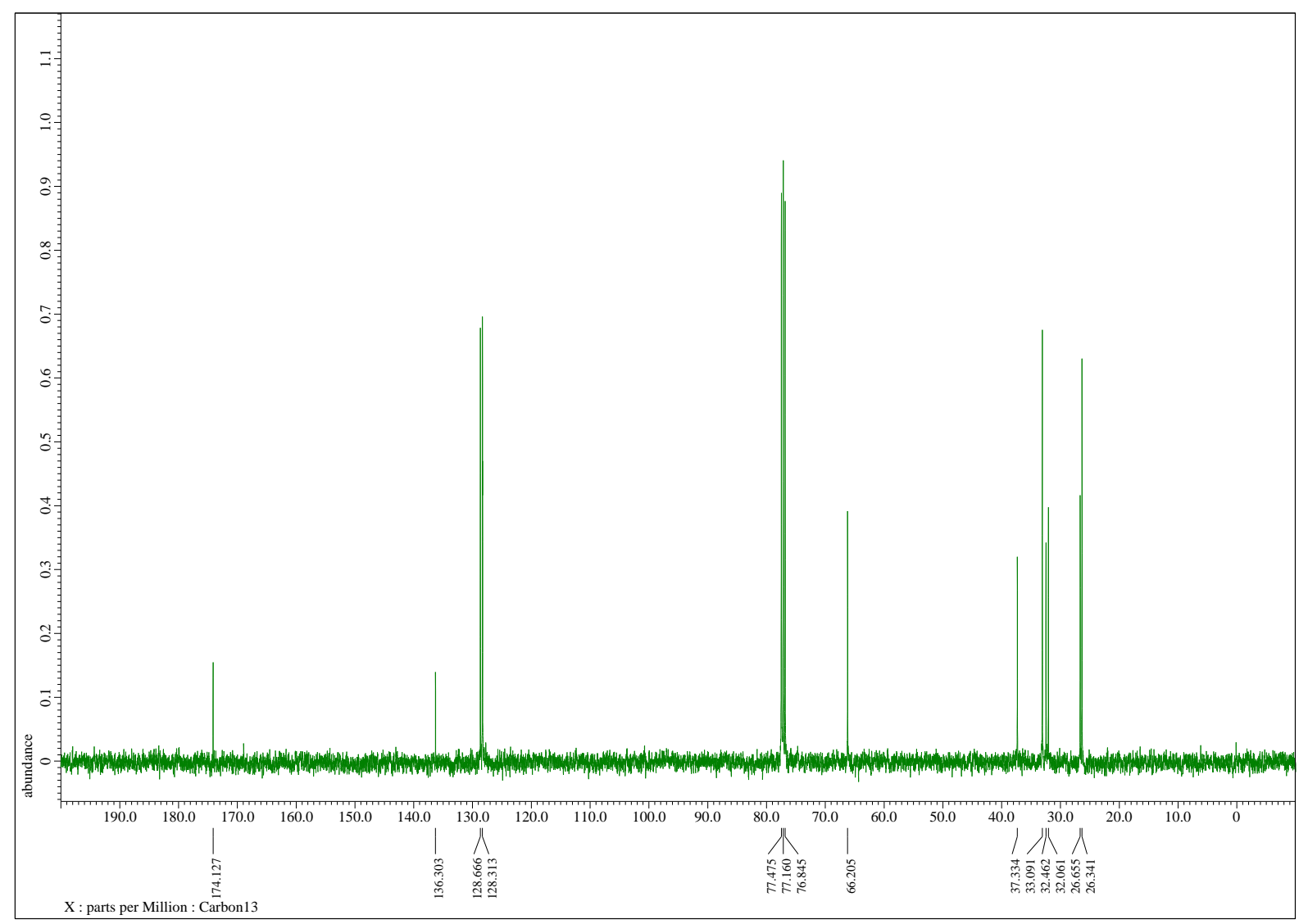


${ }^{1} \mathrm{H}$ NMR spectrum of $21\left(400 \mathrm{MHz}, \mathrm{CDCl}_{3}\right)$

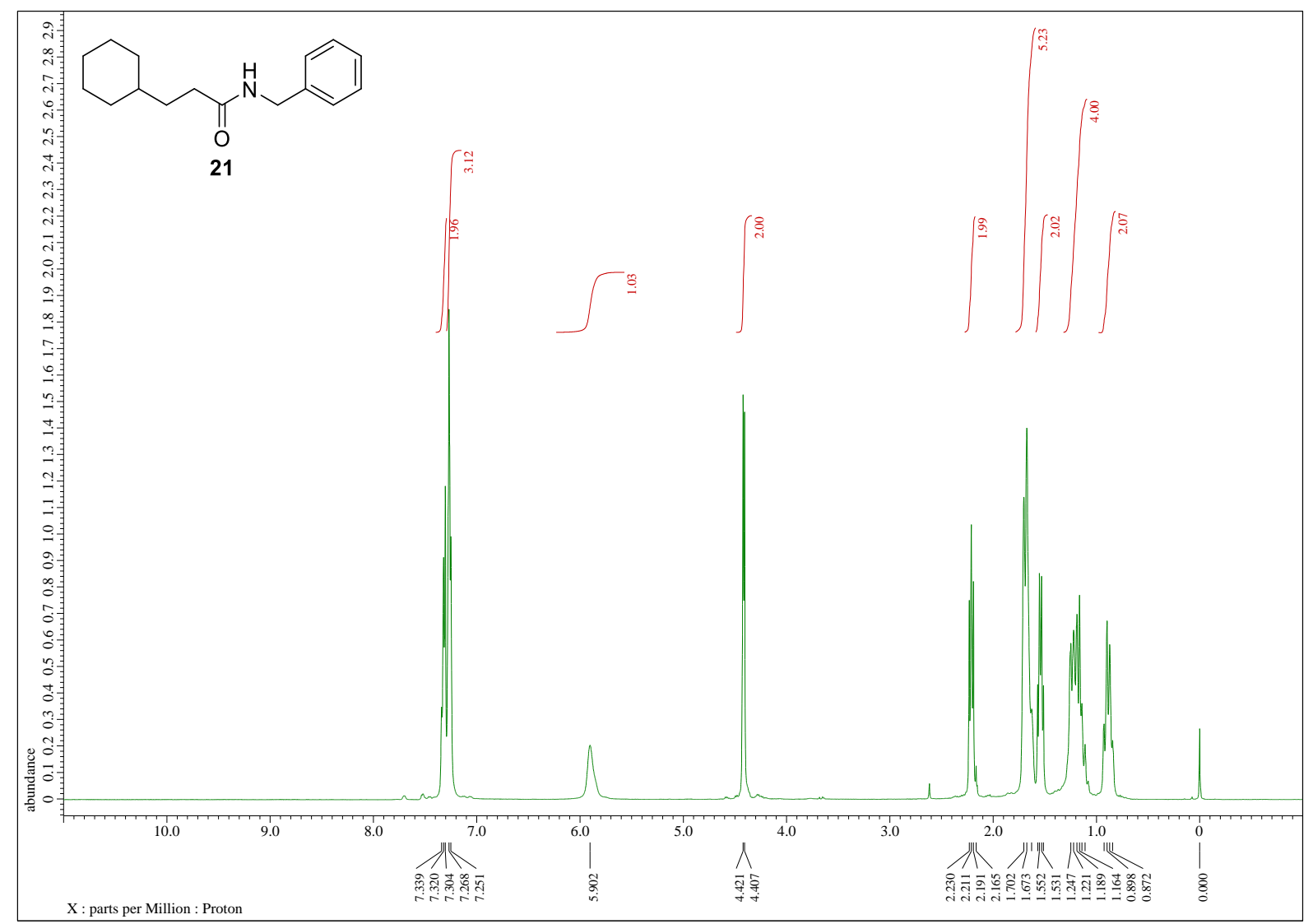

${ }^{13} \mathrm{C}$ NMR spectrum of $21\left(100 \mathrm{MHz}, \mathrm{CDCl}_{3}\right)$

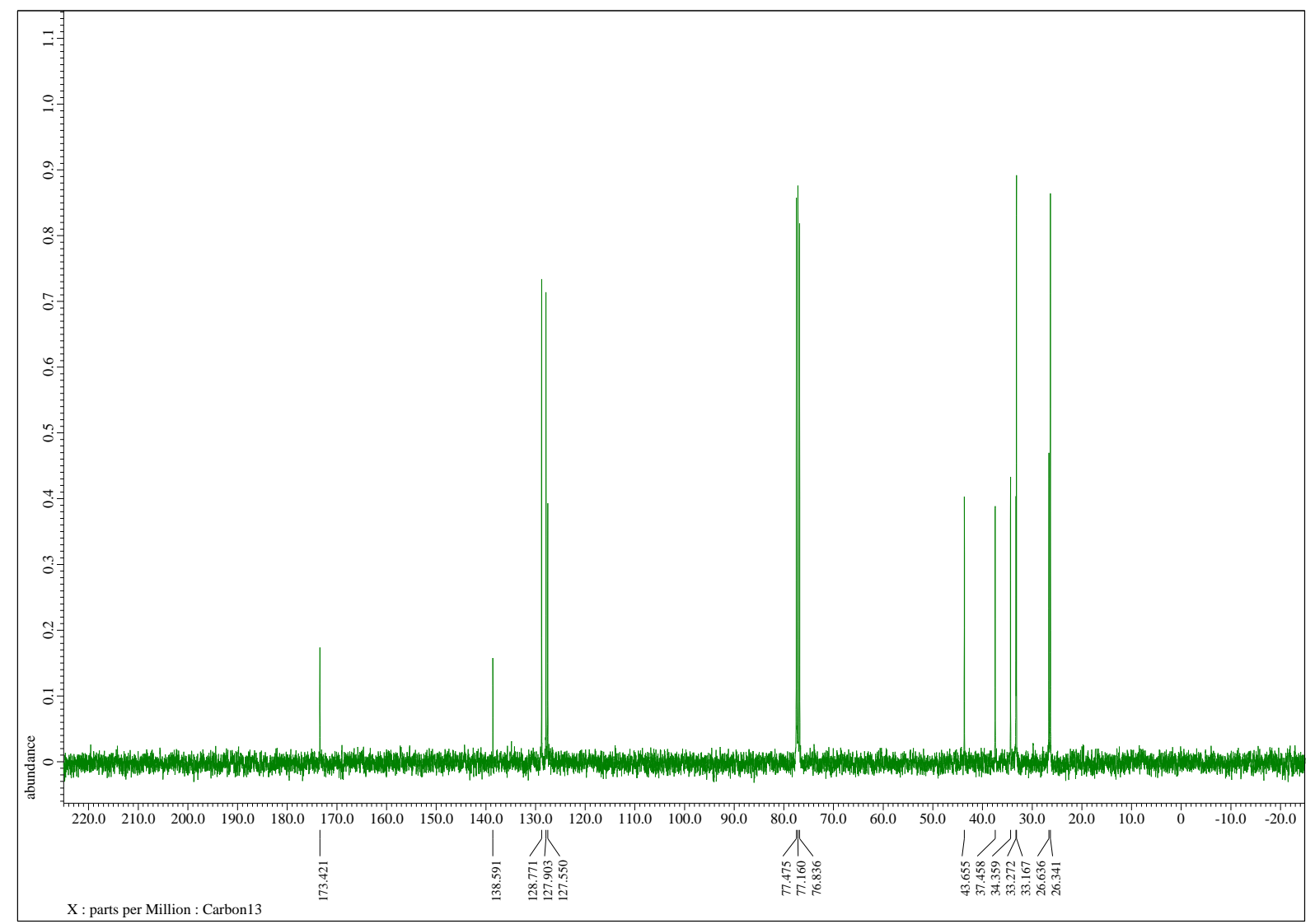


${ }^{1} \mathrm{H}$ NMR spectrum of $22\left(400 \mathrm{MHz}, \mathrm{CDCl}_{3}\right)$

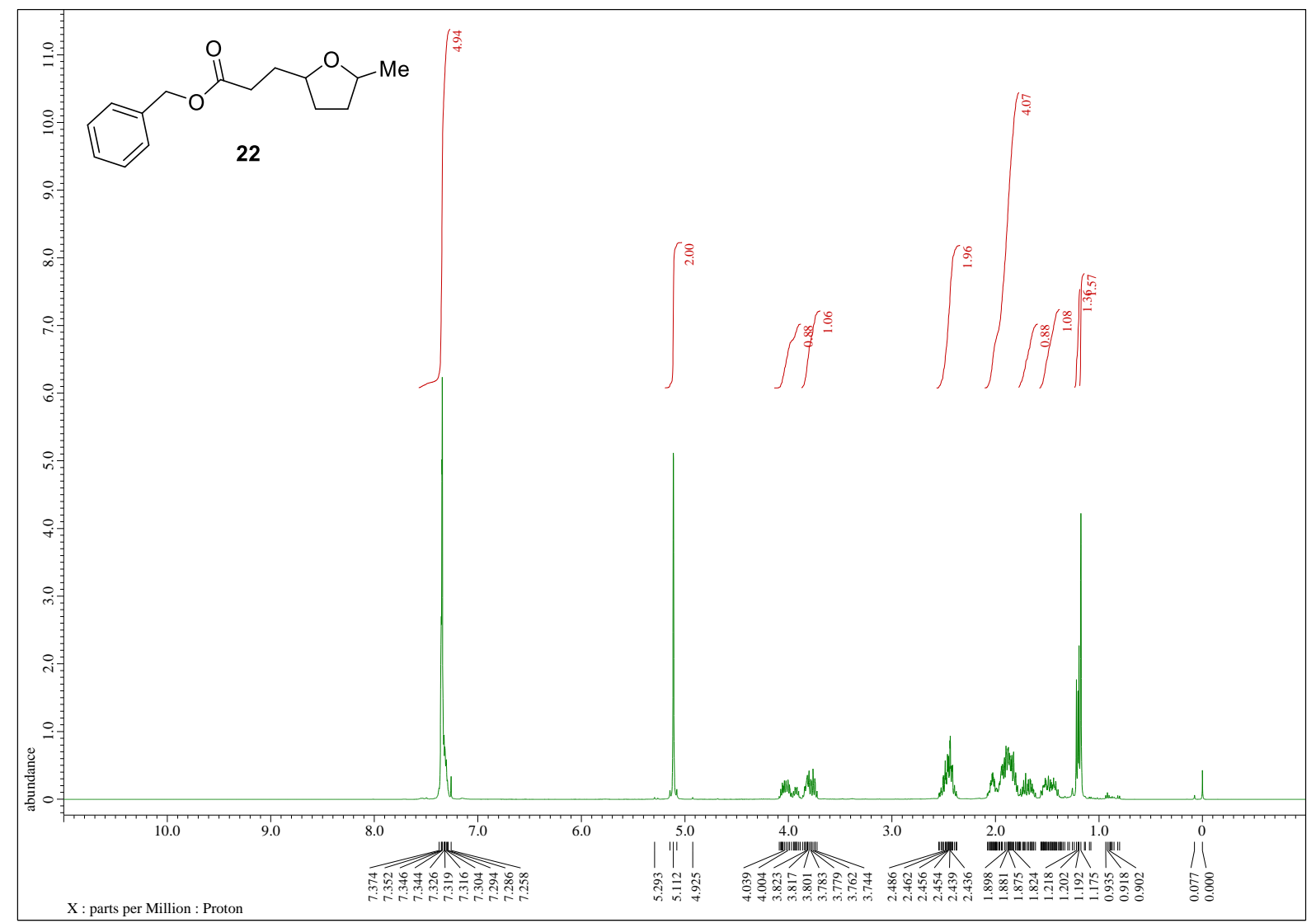

${ }^{13} \mathrm{C}$ NMR spectrum of $22\left(100 \mathrm{MHz}, \mathrm{CDCl}_{3}\right)$

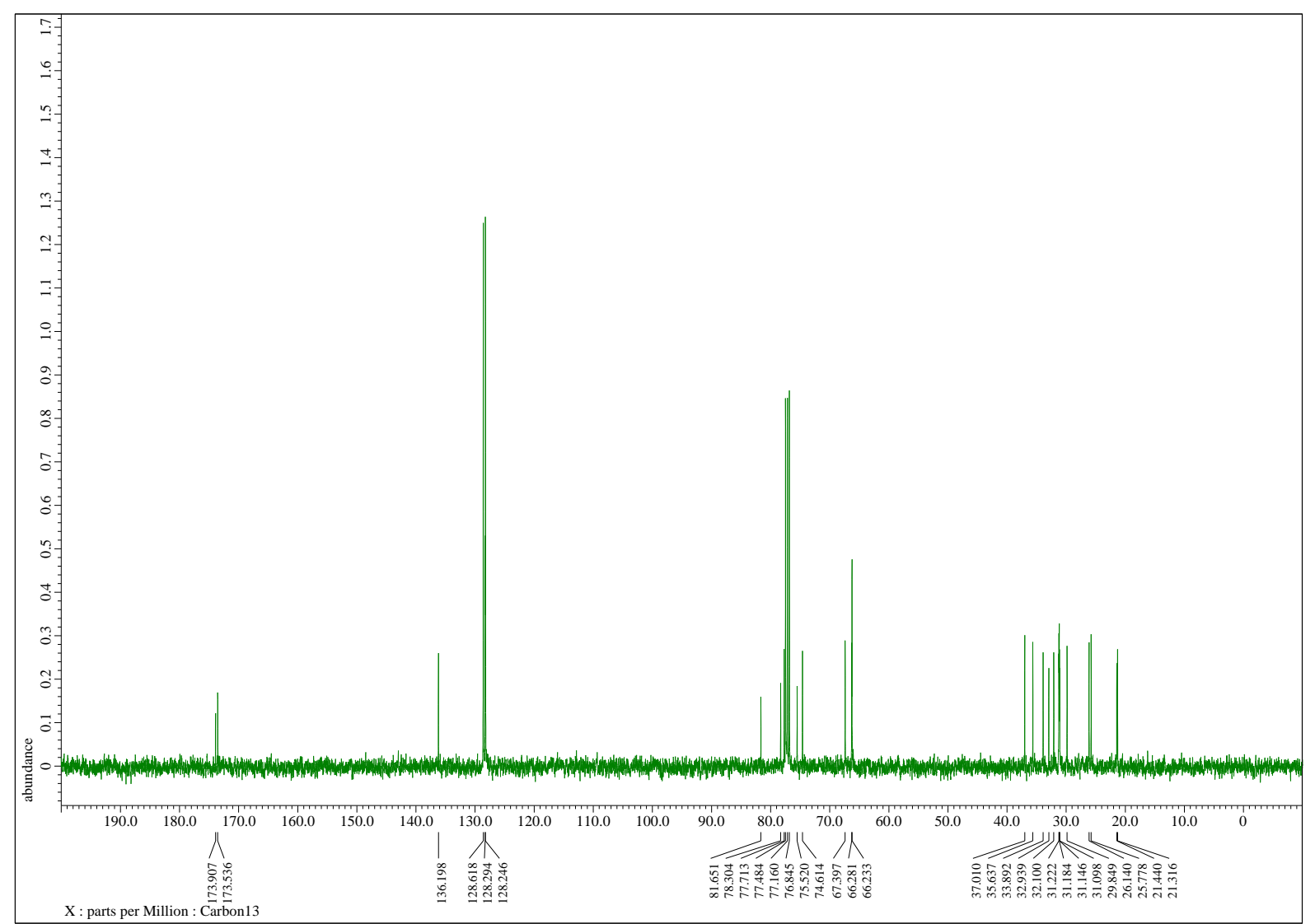


${ }^{1} \mathrm{H}$ NMR spectrum of $23\left(400 \mathrm{MHz}, \mathrm{CDCl}_{3}\right)$

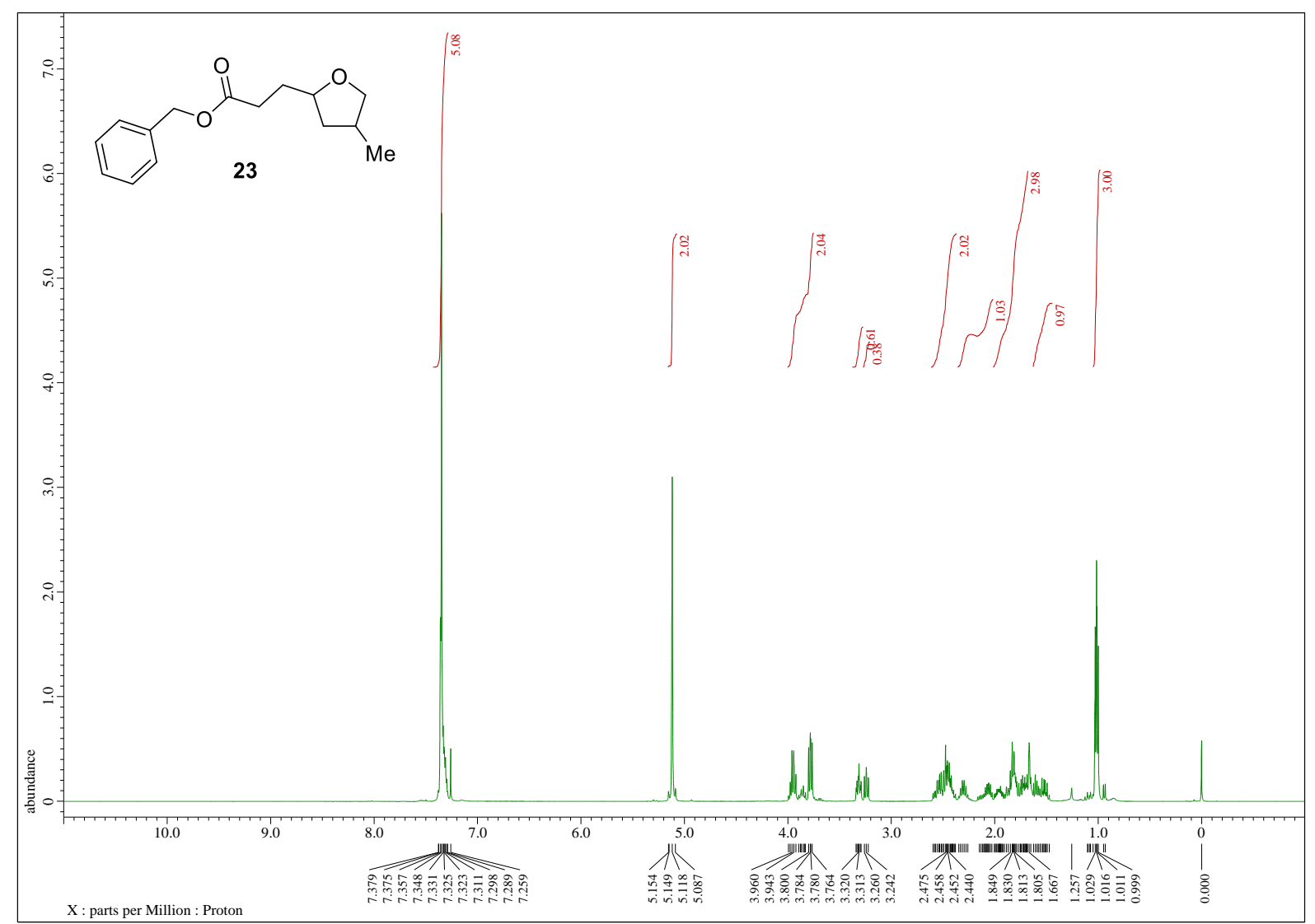

${ }^{13} \mathrm{C}$ NMR spectrum of $23\left(100 \mathrm{MHz}, \mathrm{CDCl}_{3}\right)$

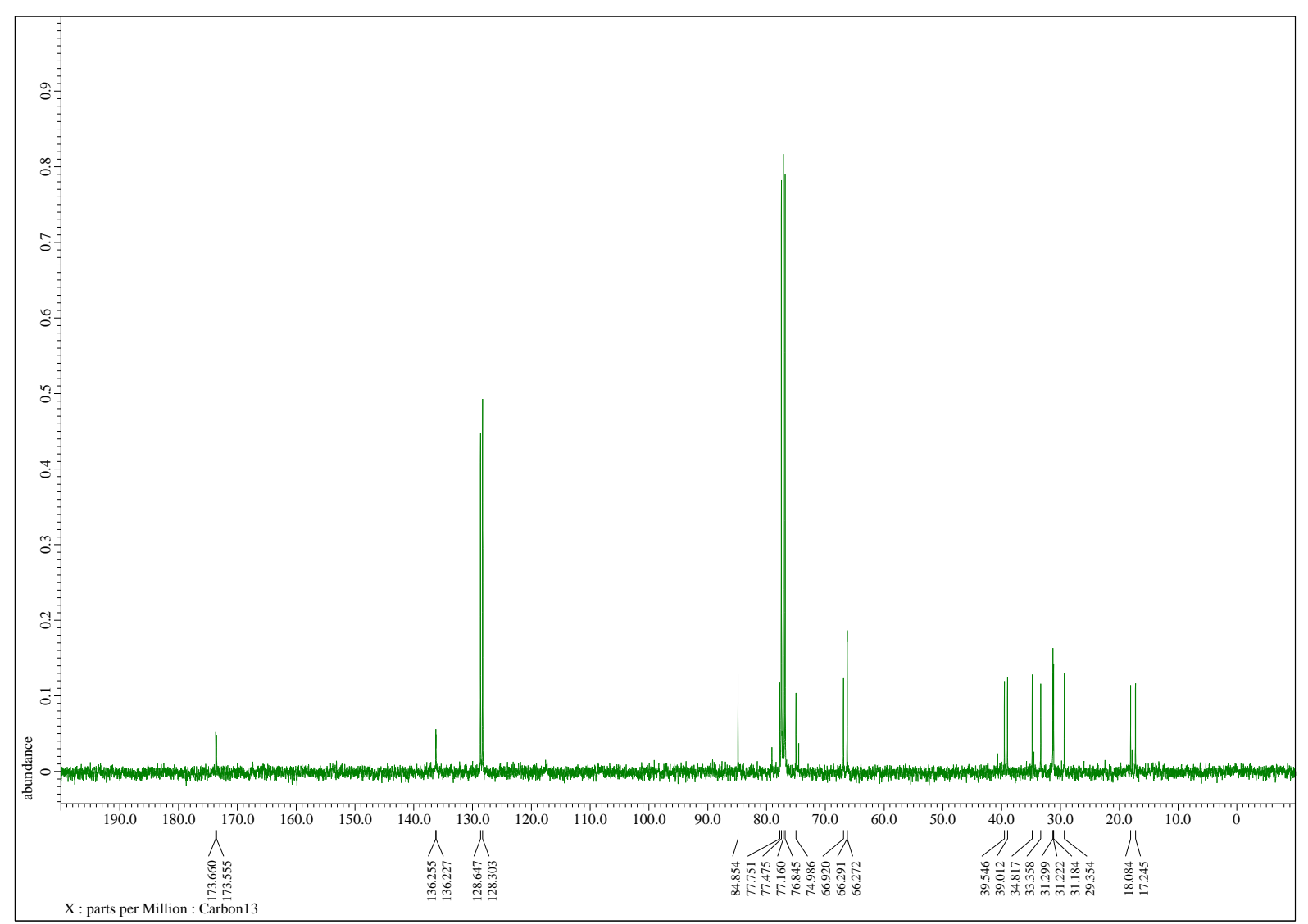


${ }^{1} \mathrm{H}$ NMR spectrum of $24\left(400 \mathrm{MHz}, \mathrm{CDCl}_{3}\right)$

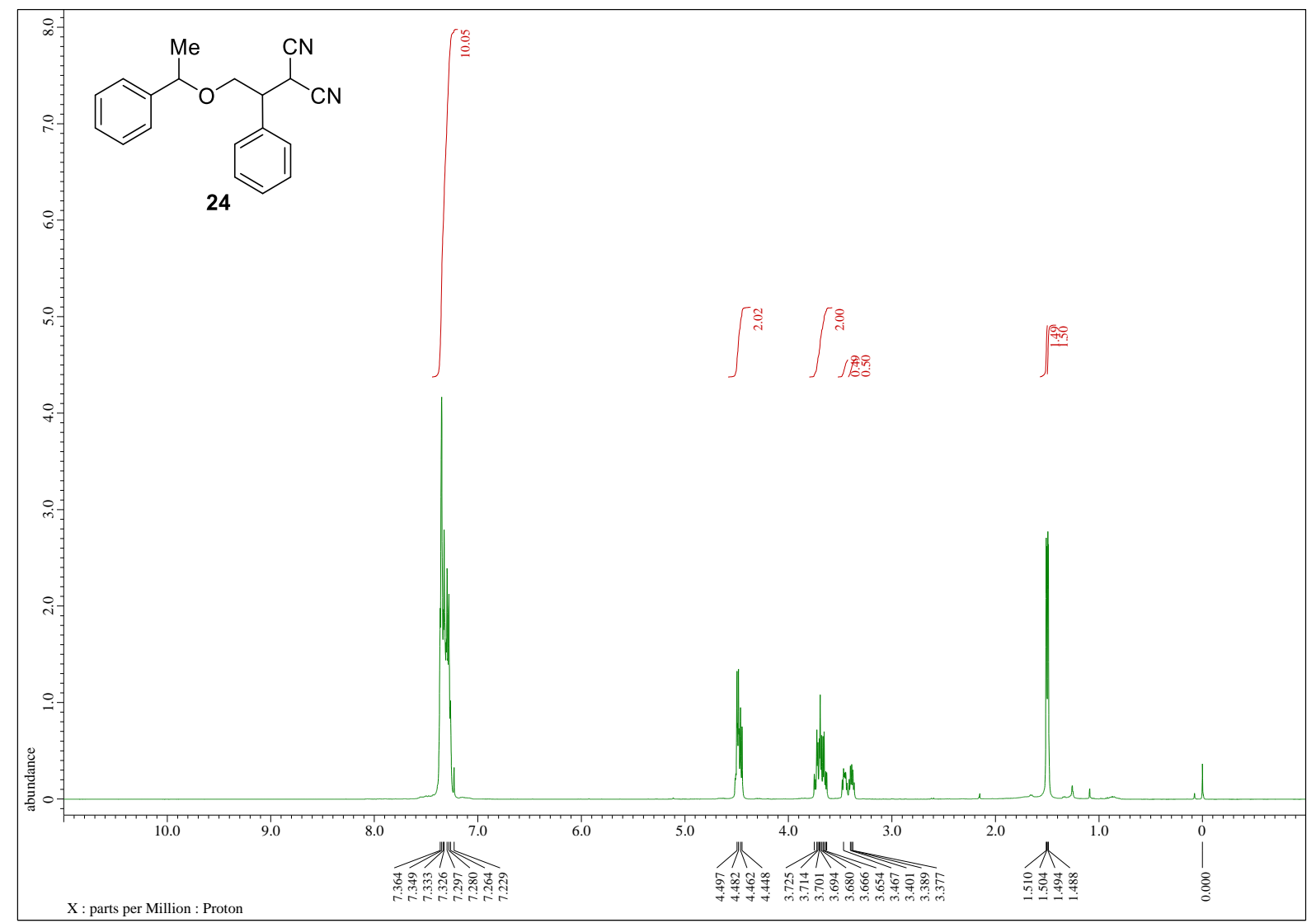

${ }^{13} \mathrm{C}$ NMR spectrum of $24\left(100 \mathrm{MHz}, \mathrm{CDCl}_{3}\right)$

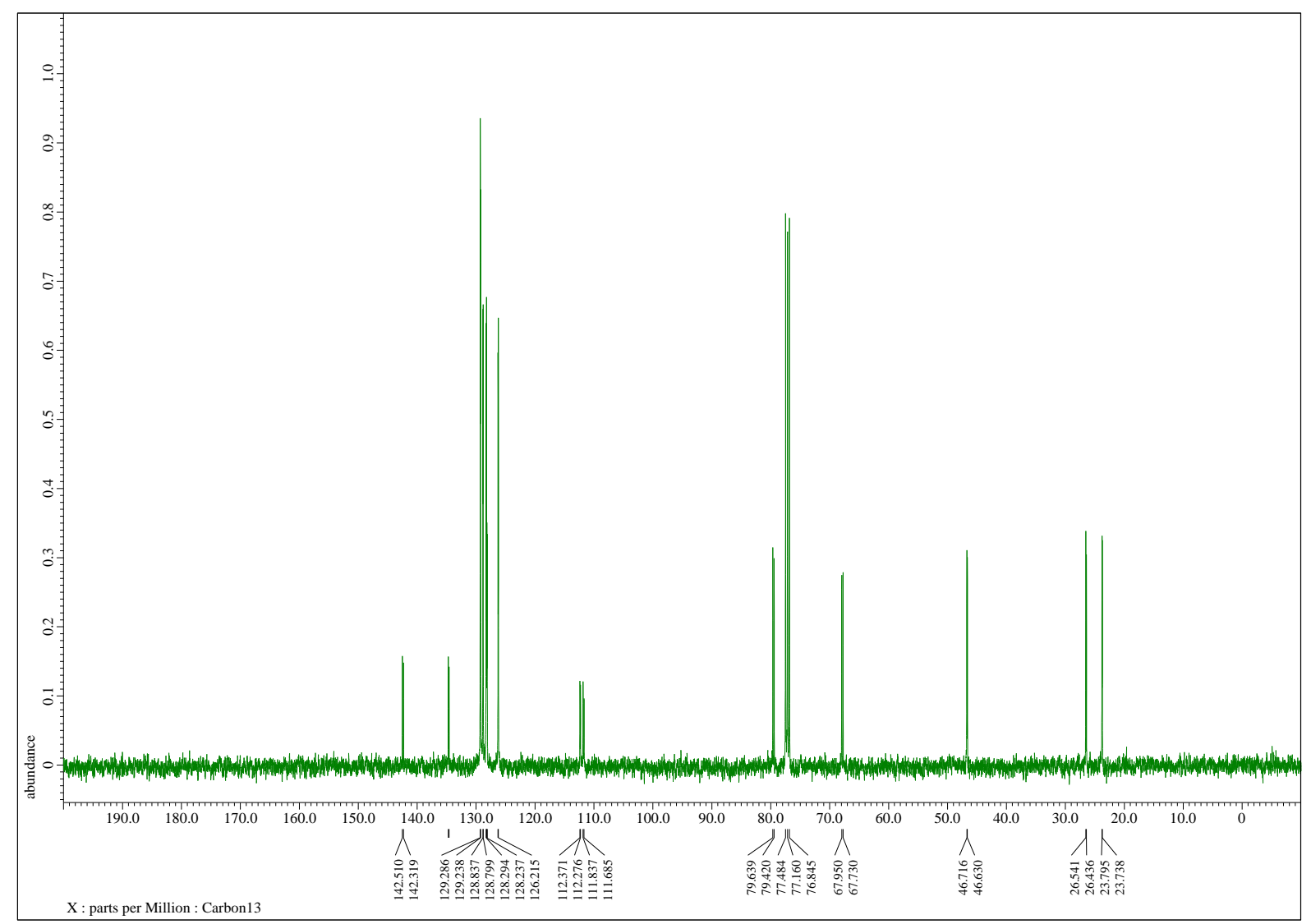


${ }^{1} \mathrm{H}$ NMR spectrum of $25\left(400 \mathrm{MHz}, \mathrm{CDCl}_{3}\right)$

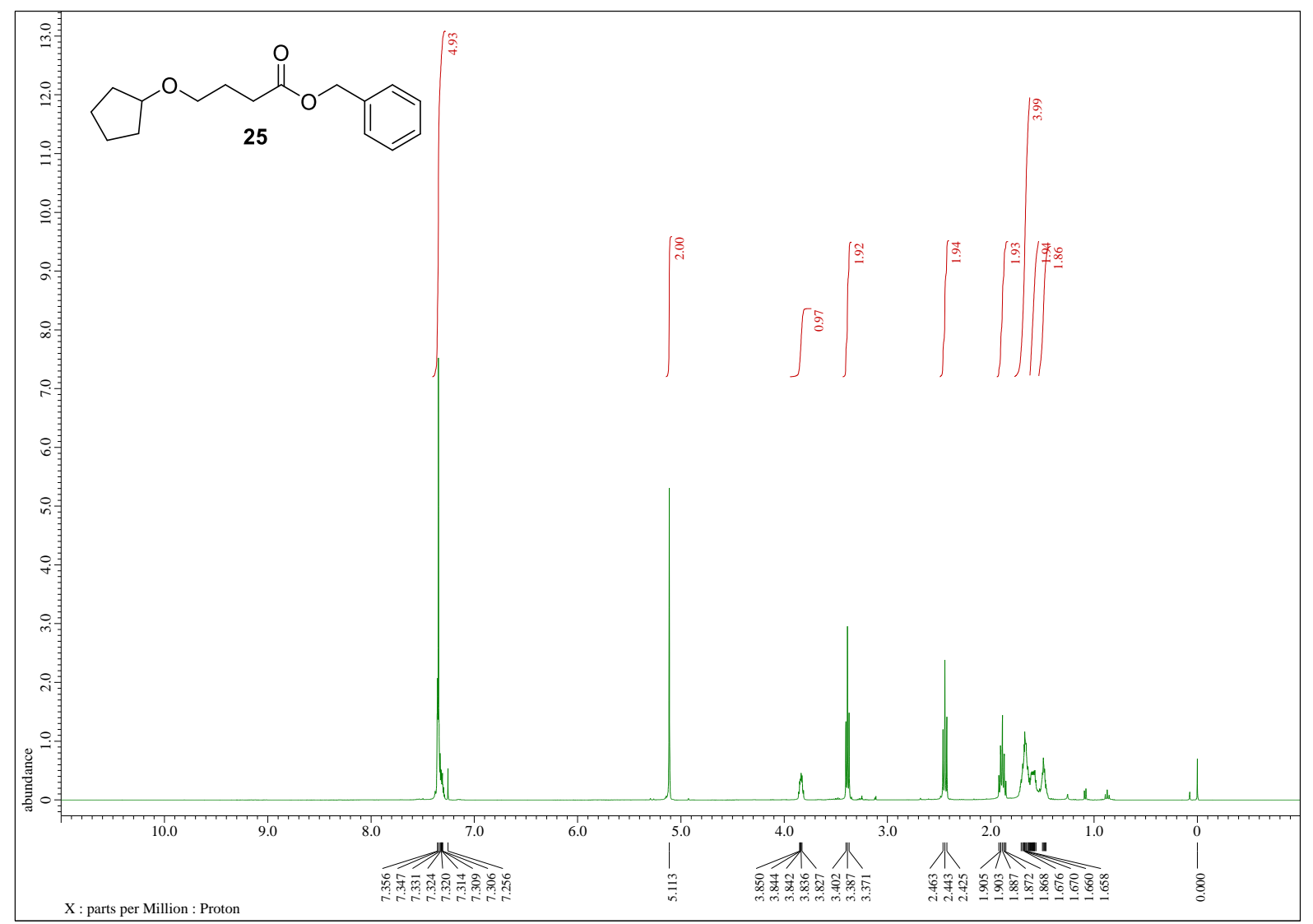

${ }^{13} \mathrm{C}$ NMR spectrum of $25\left(100 \mathrm{MHz}, \mathrm{CDCl}_{3}\right)$

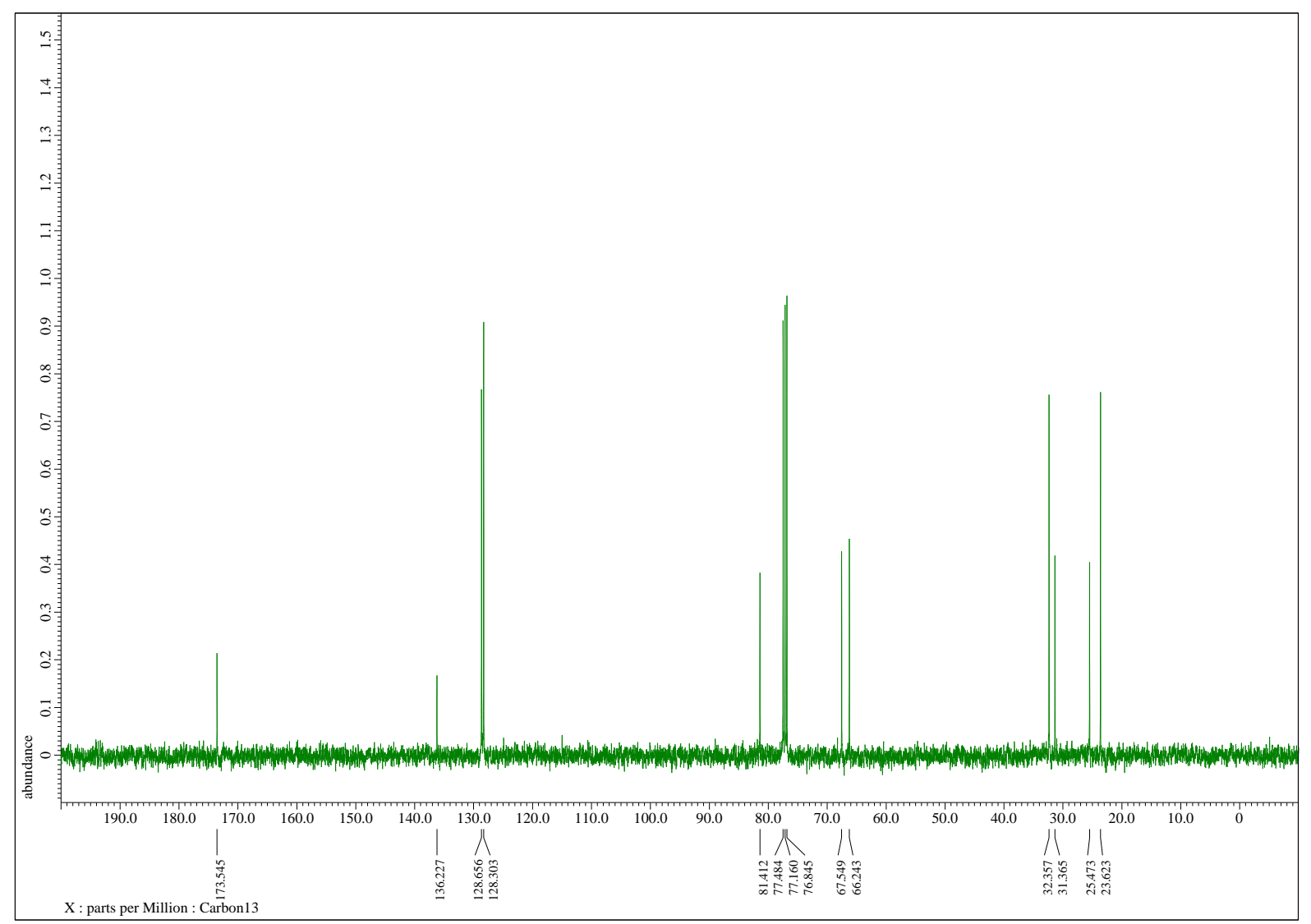


${ }^{1} \mathrm{H}$ NMR spectrum of $26\left(400 \mathrm{MHz}, \mathrm{CDCl}_{3}\right)$

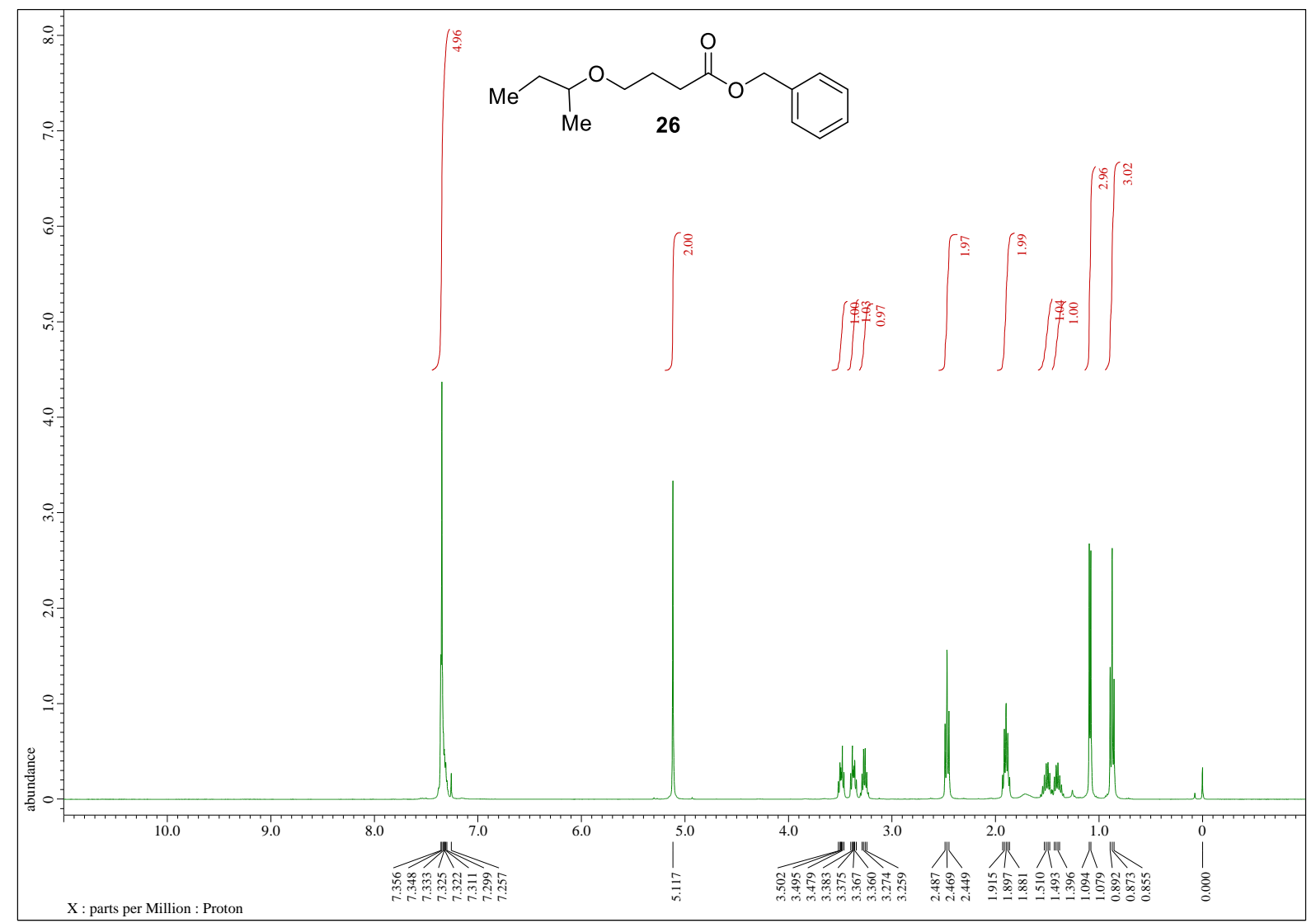

${ }^{13} \mathrm{C}$ NMR spectrum of $26\left(100 \mathrm{MHz}, \mathrm{CDCl}_{3}\right)$

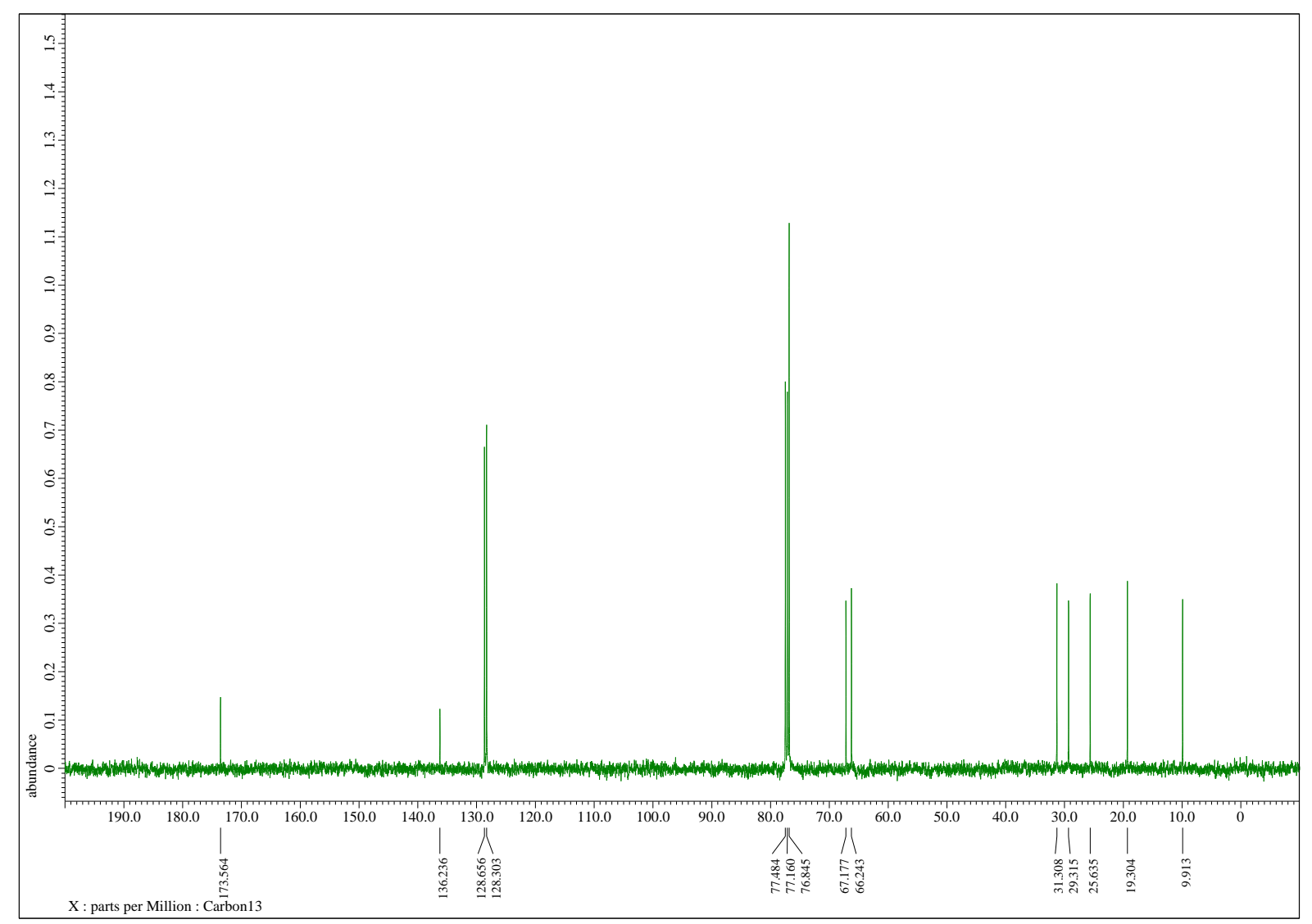


${ }^{1} \mathrm{H}$ NMR spectrum of $27\left(400 \mathrm{MHz}, \mathrm{CDCl}_{3}\right)$

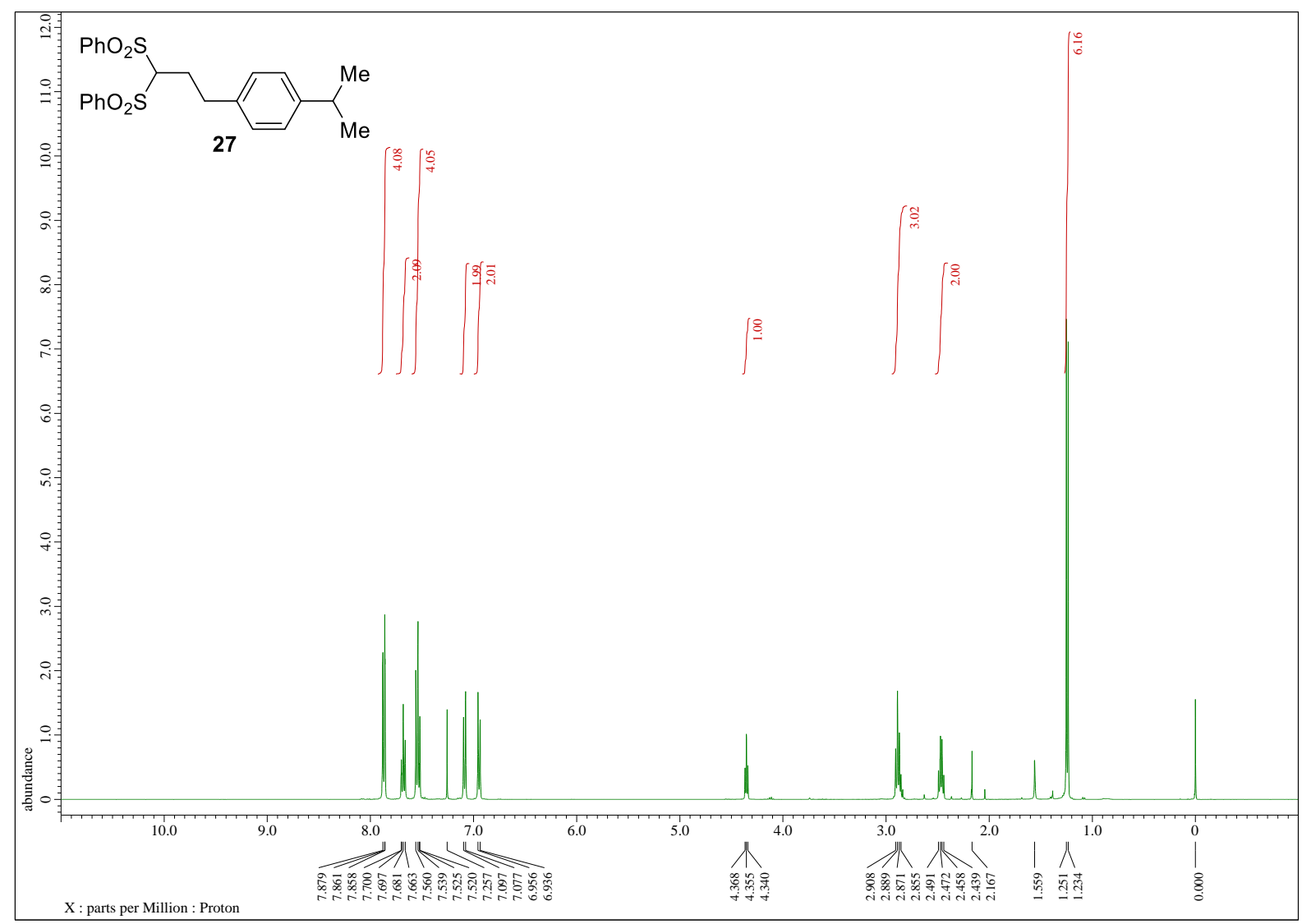

${ }^{13} \mathrm{C}$ NMR spectrum of $27\left(100 \mathrm{MHz}, \mathrm{CDCl}_{3}\right)$

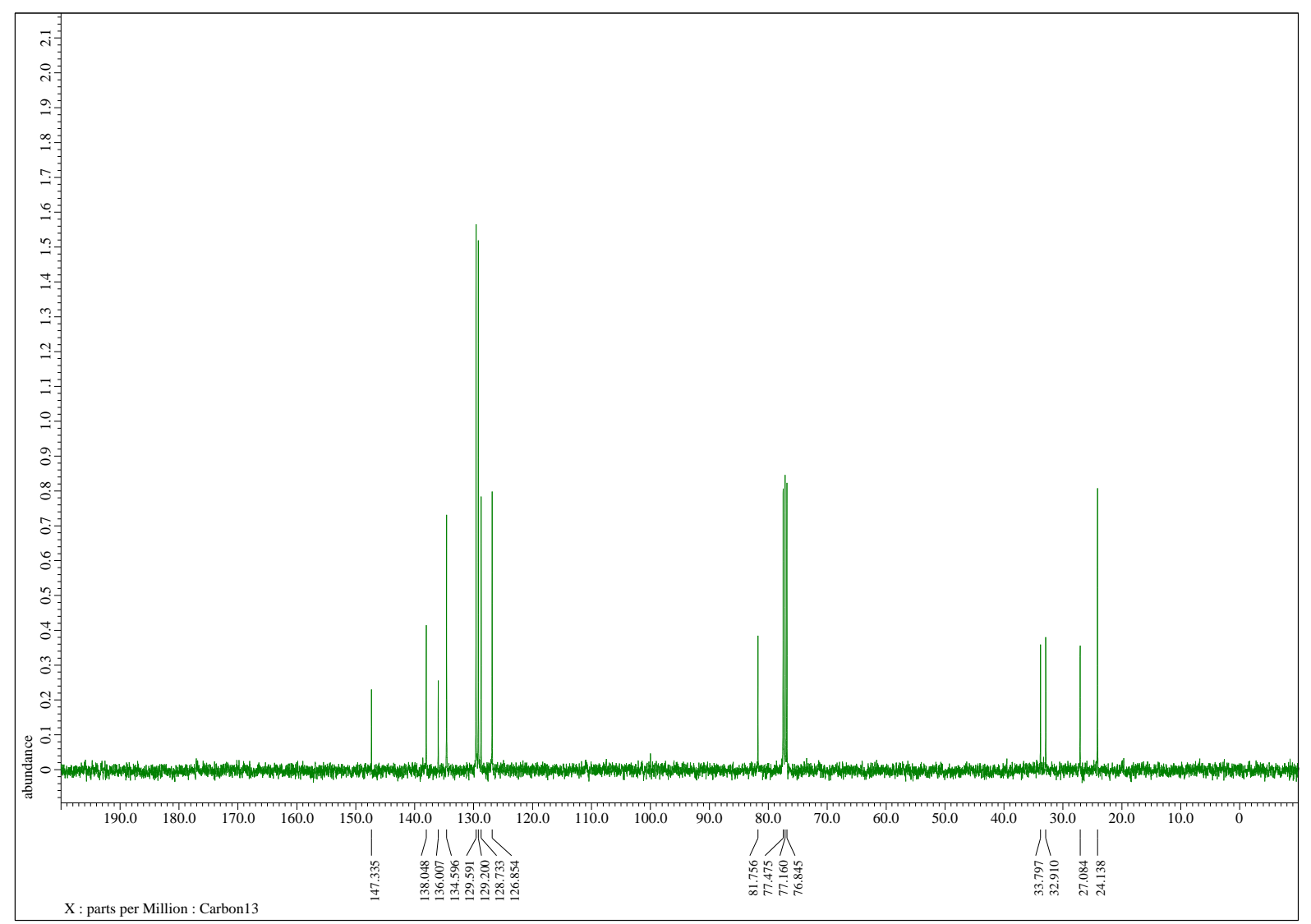


${ }^{1} \mathrm{H}$ NMR spectrum of $28\left(400 \mathrm{MHz}, \mathrm{CDCl}_{3}\right)$

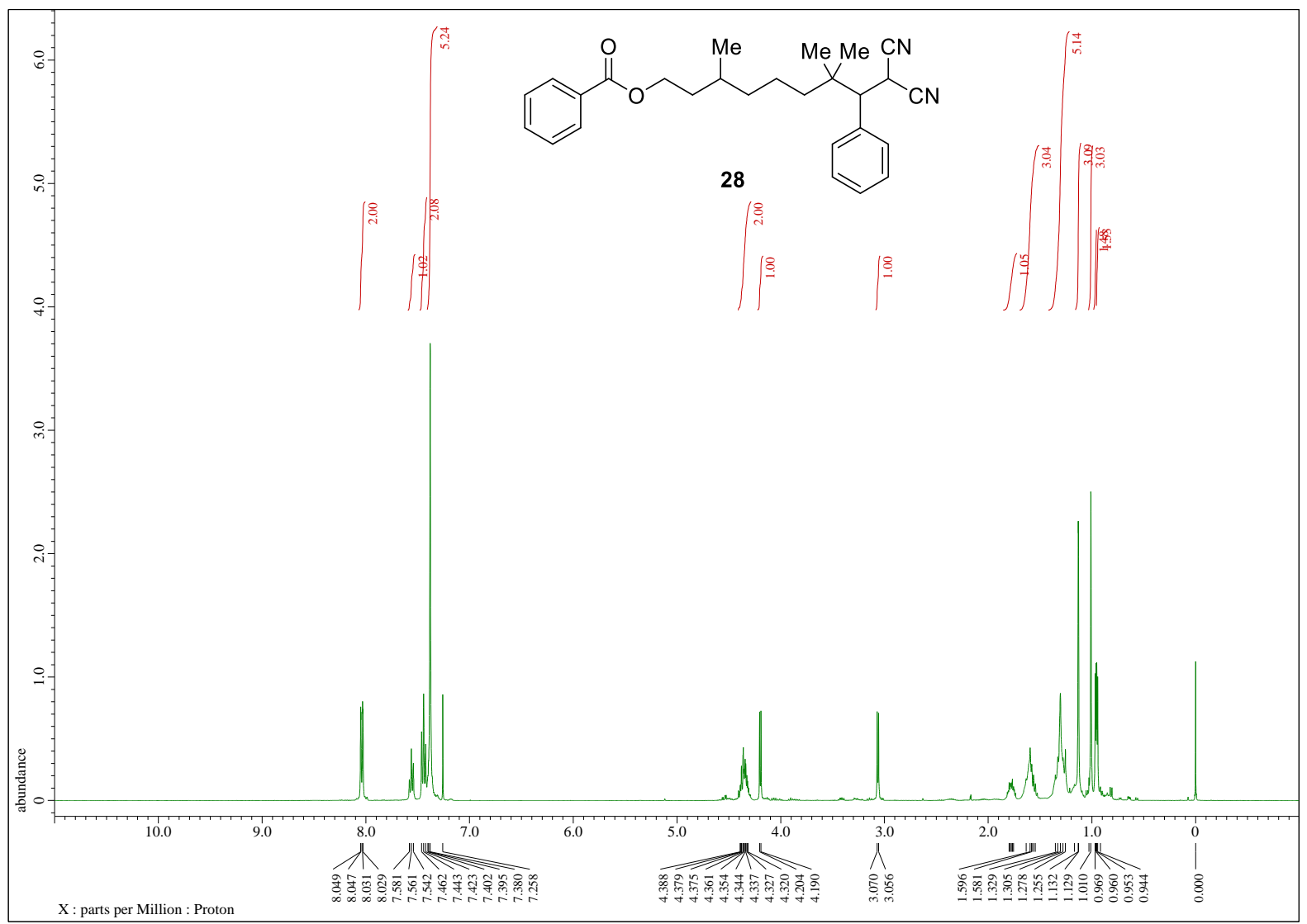

${ }^{13} \mathrm{C}$ NMR spectrum of $28\left(100 \mathrm{MHz}, \mathrm{CDCl}_{3}\right)$

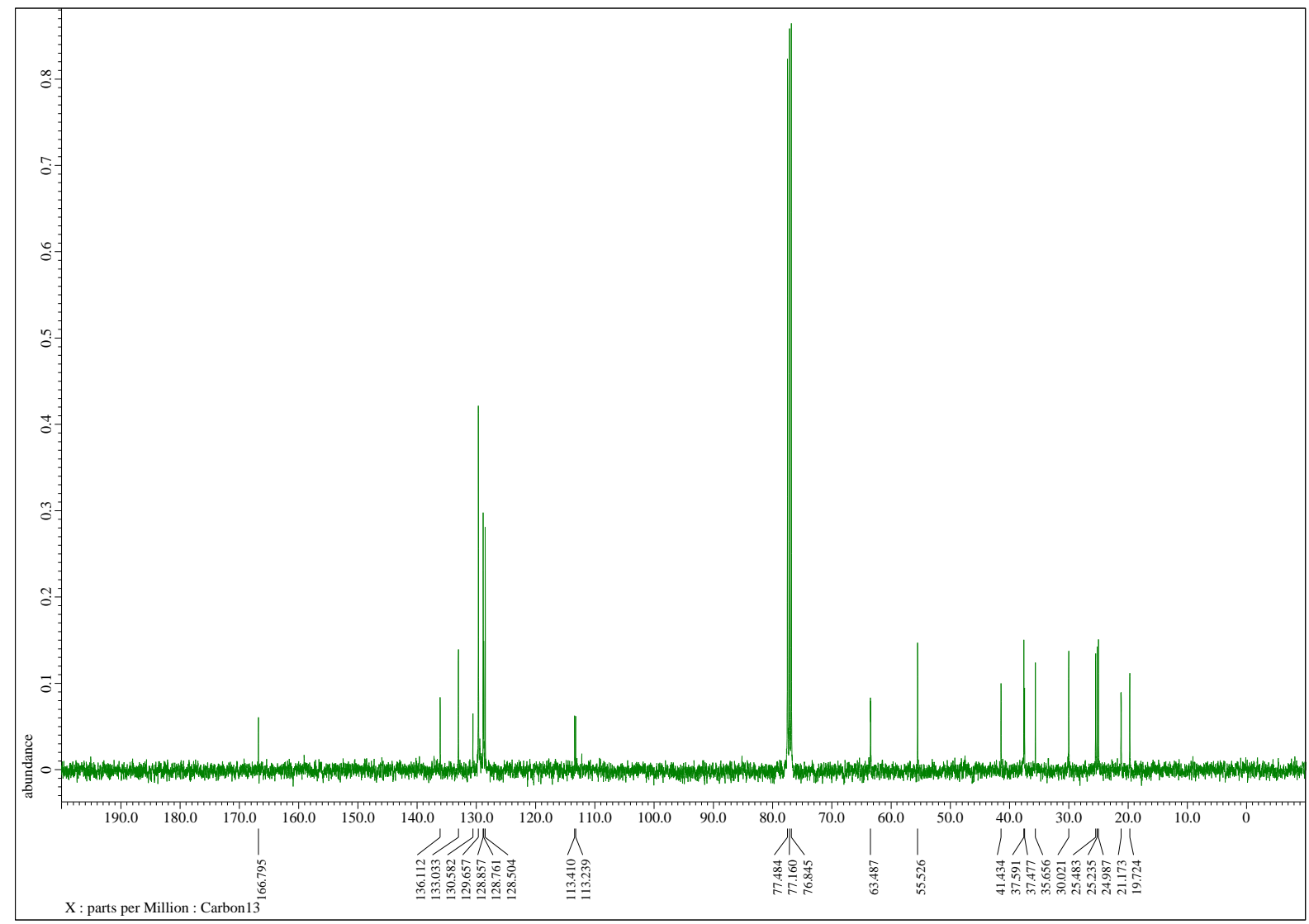


${ }^{1} \mathrm{H}$ NMR spectrum of $29\left(400 \mathrm{MHz}, \mathrm{CDCl}_{3}\right)$

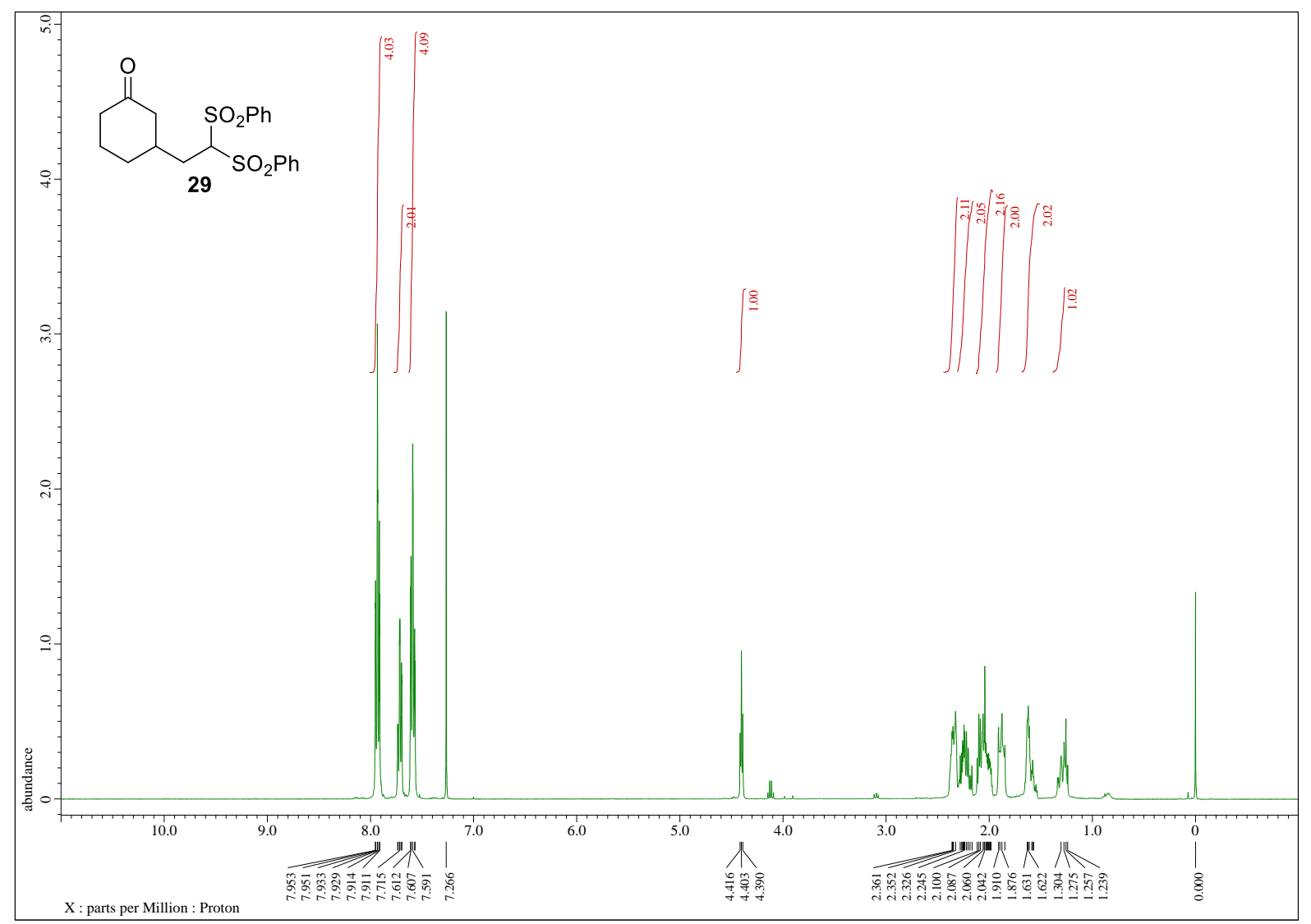

${ }^{13} \mathrm{C}$ NMR spectrum of $29\left(100 \mathrm{MHz}, \mathrm{CDCl}_{3}\right)$

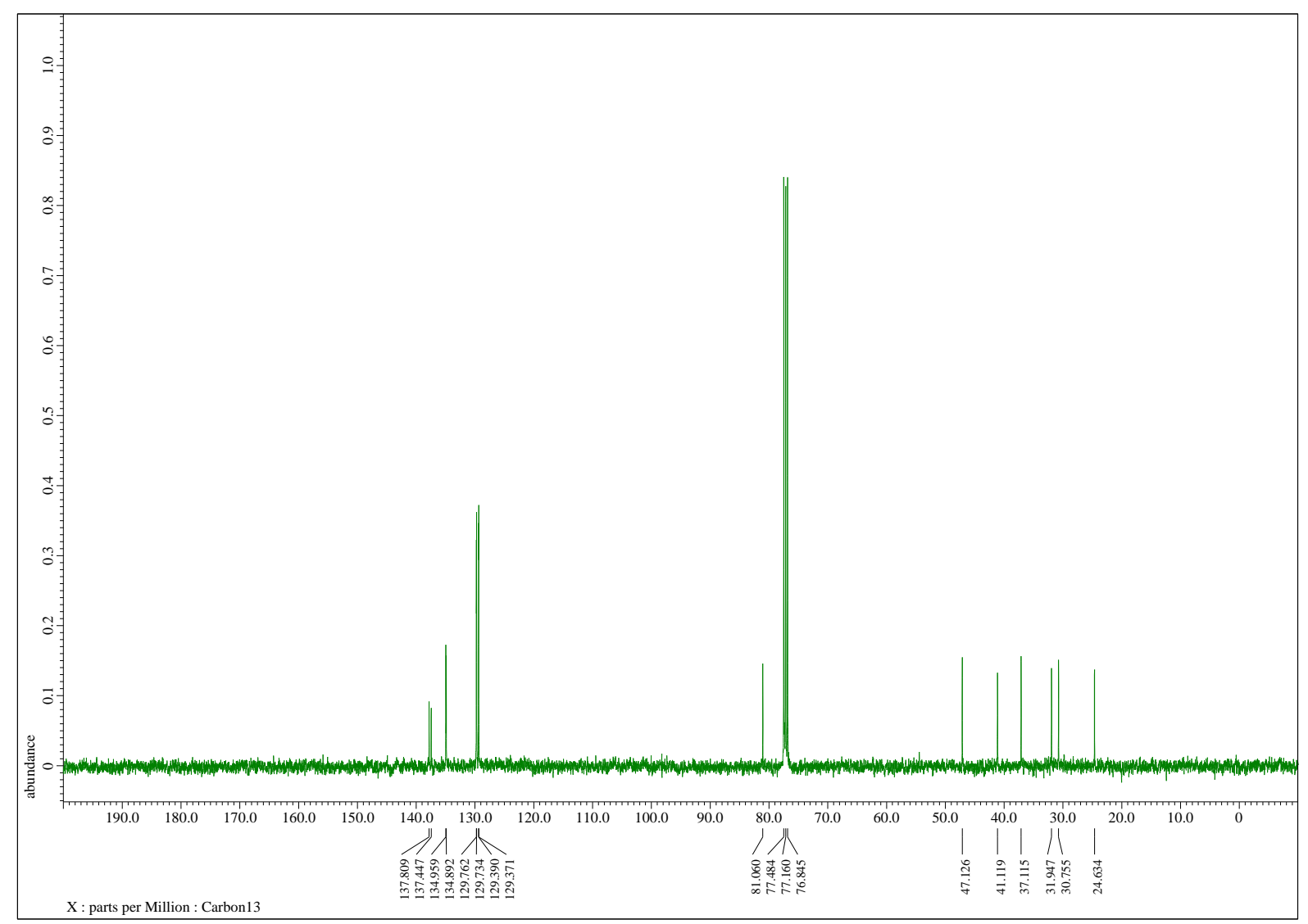


${ }^{1} \mathrm{H}$ NMR spectrum of $\mathbf{3 0}\left(400 \mathrm{MHz}, \mathrm{CDCl}_{3}\right)$

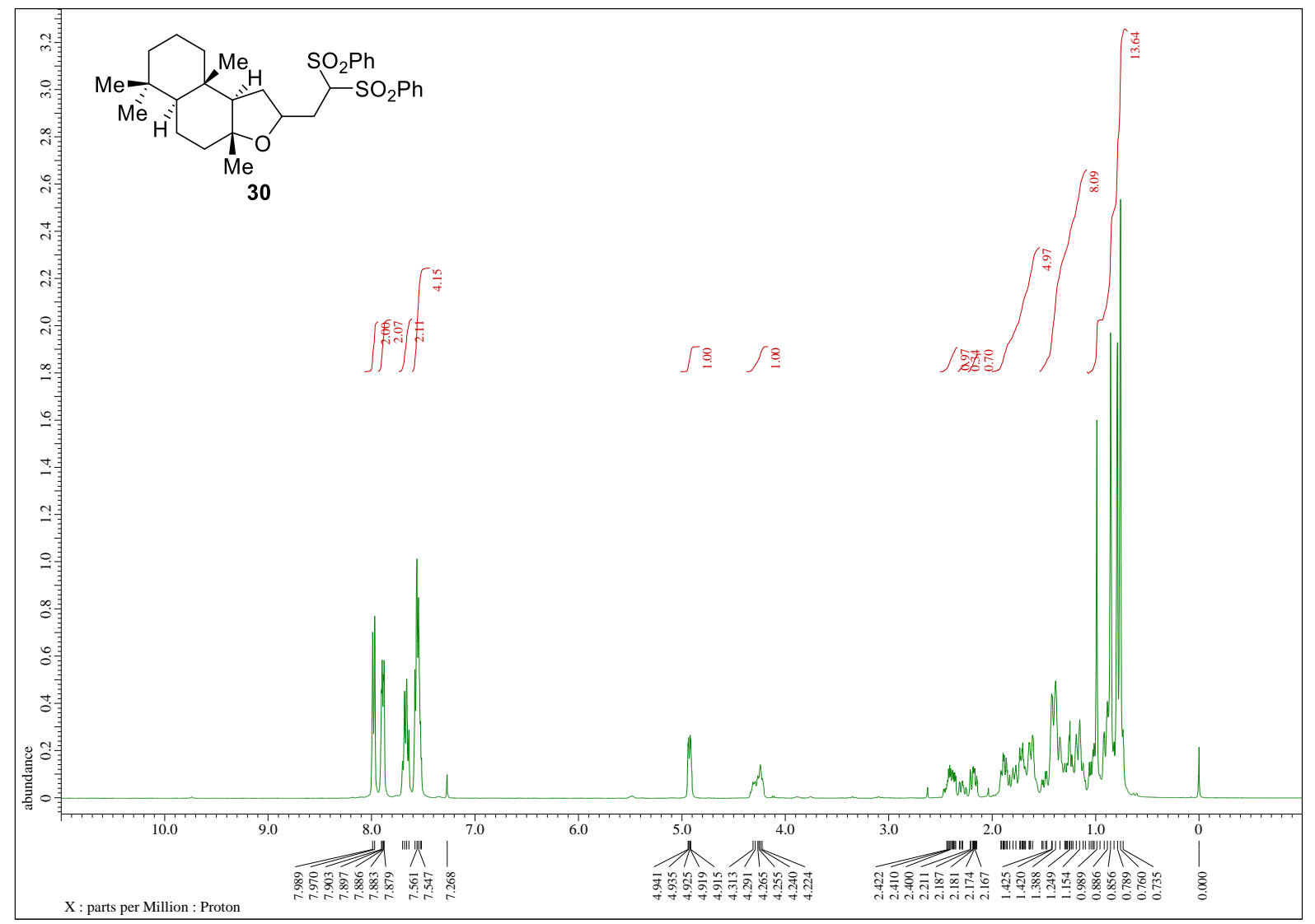

${ }^{13} \mathrm{C}$ NMR spectrum of $\mathbf{3 0}\left(100 \mathrm{MHz}, \mathrm{CDCl}_{3}\right)$

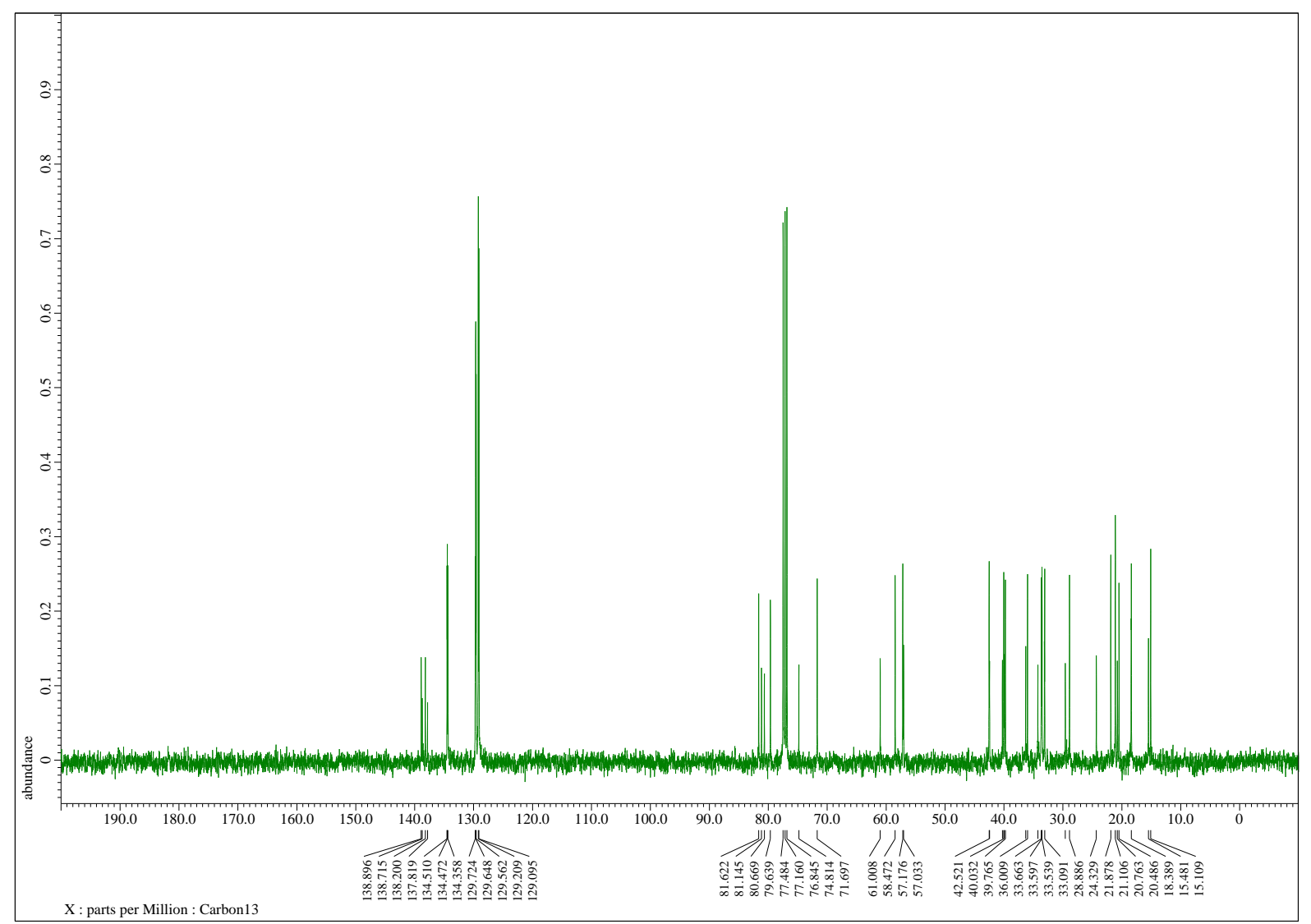


${ }^{1} \mathrm{H}$ NMR spectrum of $\mathbf{3 1}\left(400 \mathrm{MHz}, \mathrm{CDCl}_{3}\right)$

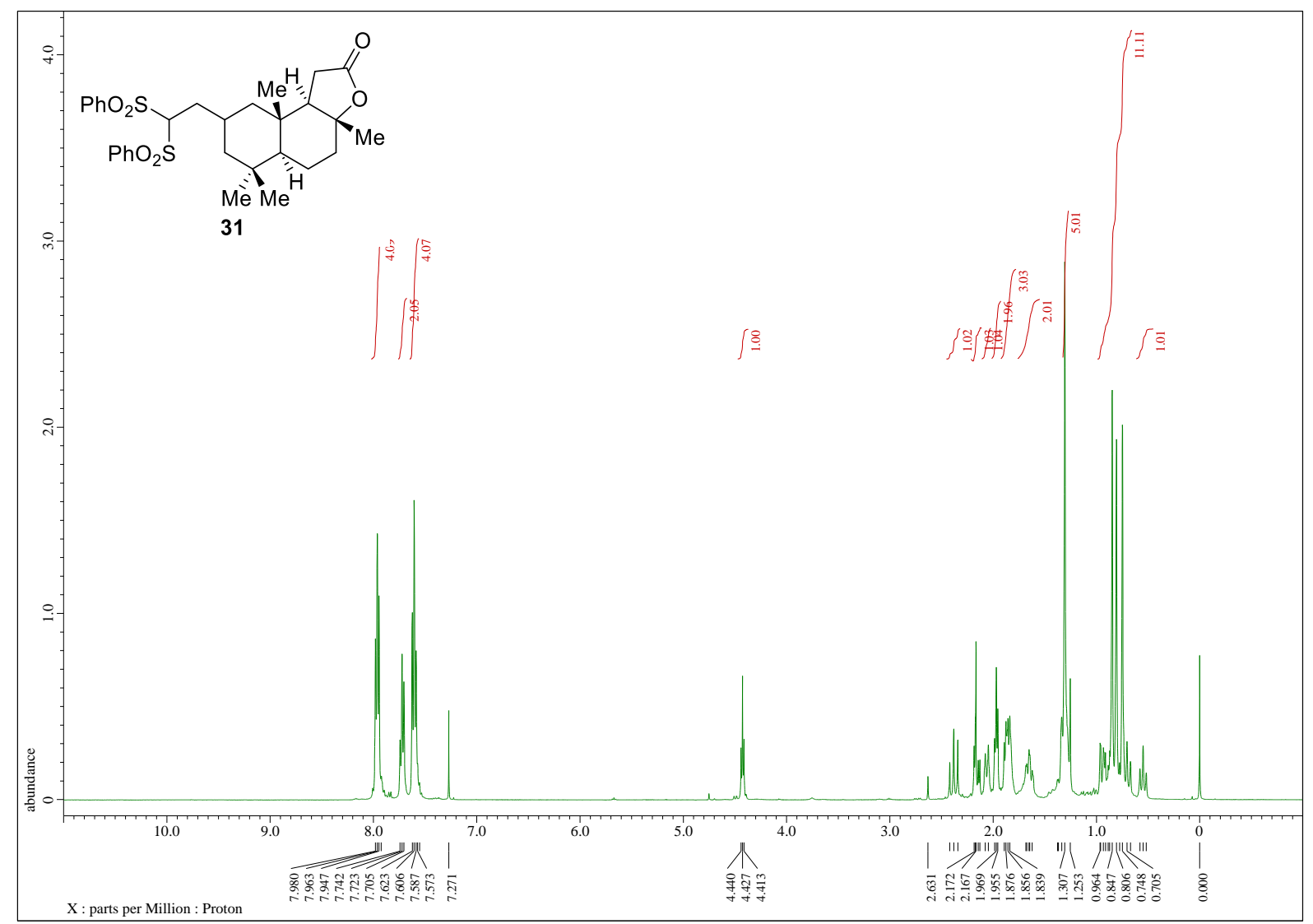

${ }^{13} \mathrm{C}$ NMR spectrum of $\mathbf{3 1}\left(100 \mathrm{MHz}, \mathrm{CDCl}_{3}\right)$

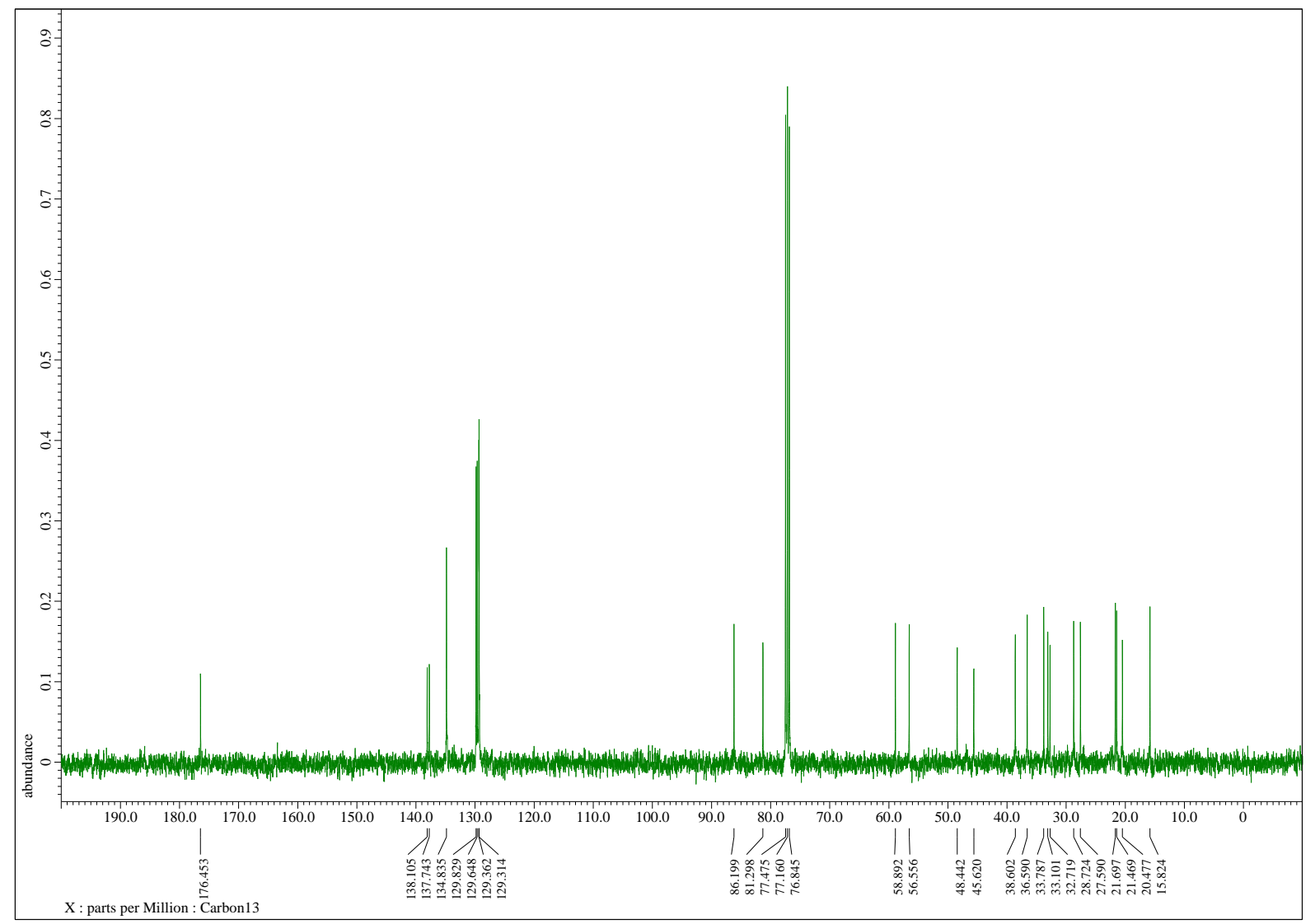


${ }^{1} \mathrm{H}$ NMR spectrum of $32\left(400 \mathrm{MHz}, \mathrm{CDCl}_{3}\right)$

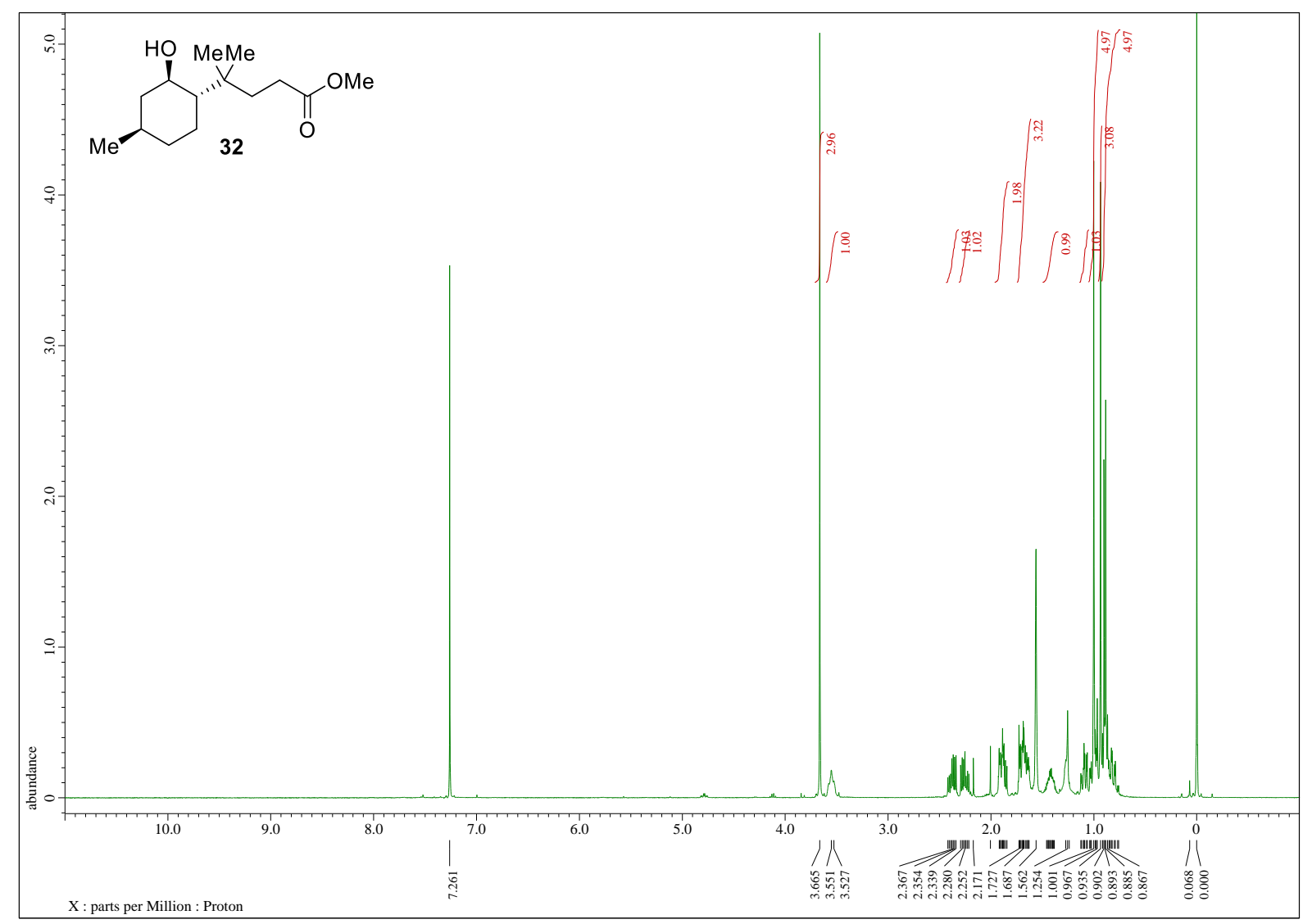

${ }^{13} \mathrm{C}$ NMR spectrum of $32\left(100 \mathrm{MHz}, \mathrm{CDCl}_{3}\right)$

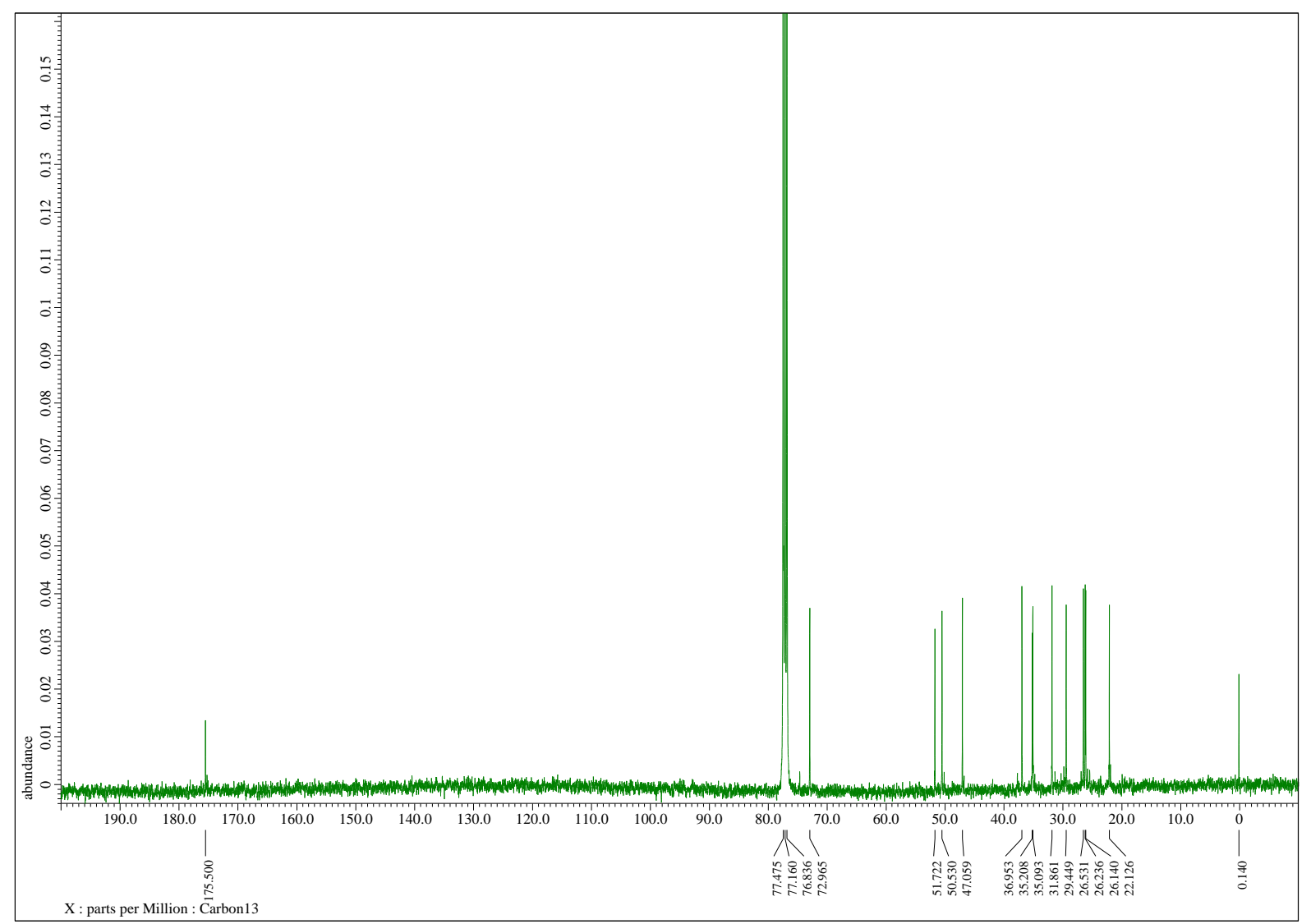


${ }^{1} \mathrm{H}$ NMR spectrum of $33\left(400 \mathrm{MHz}, \mathrm{CDCl}_{3}\right)$

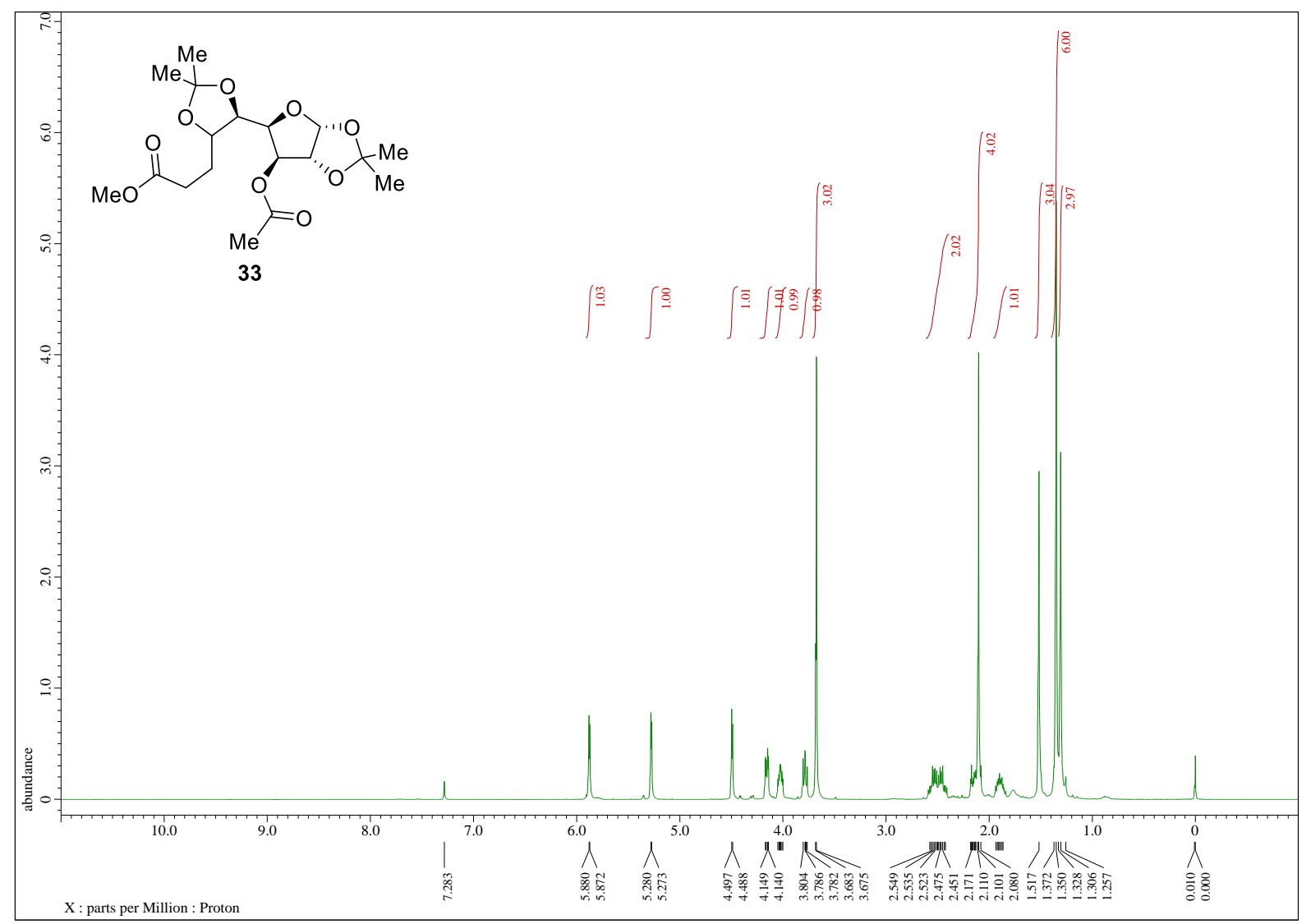

${ }^{13} \mathrm{C}$ NMR spectrum of $\mathbf{3 3}\left(100 \mathrm{MHz}, \mathrm{CDCl}_{3}\right)$

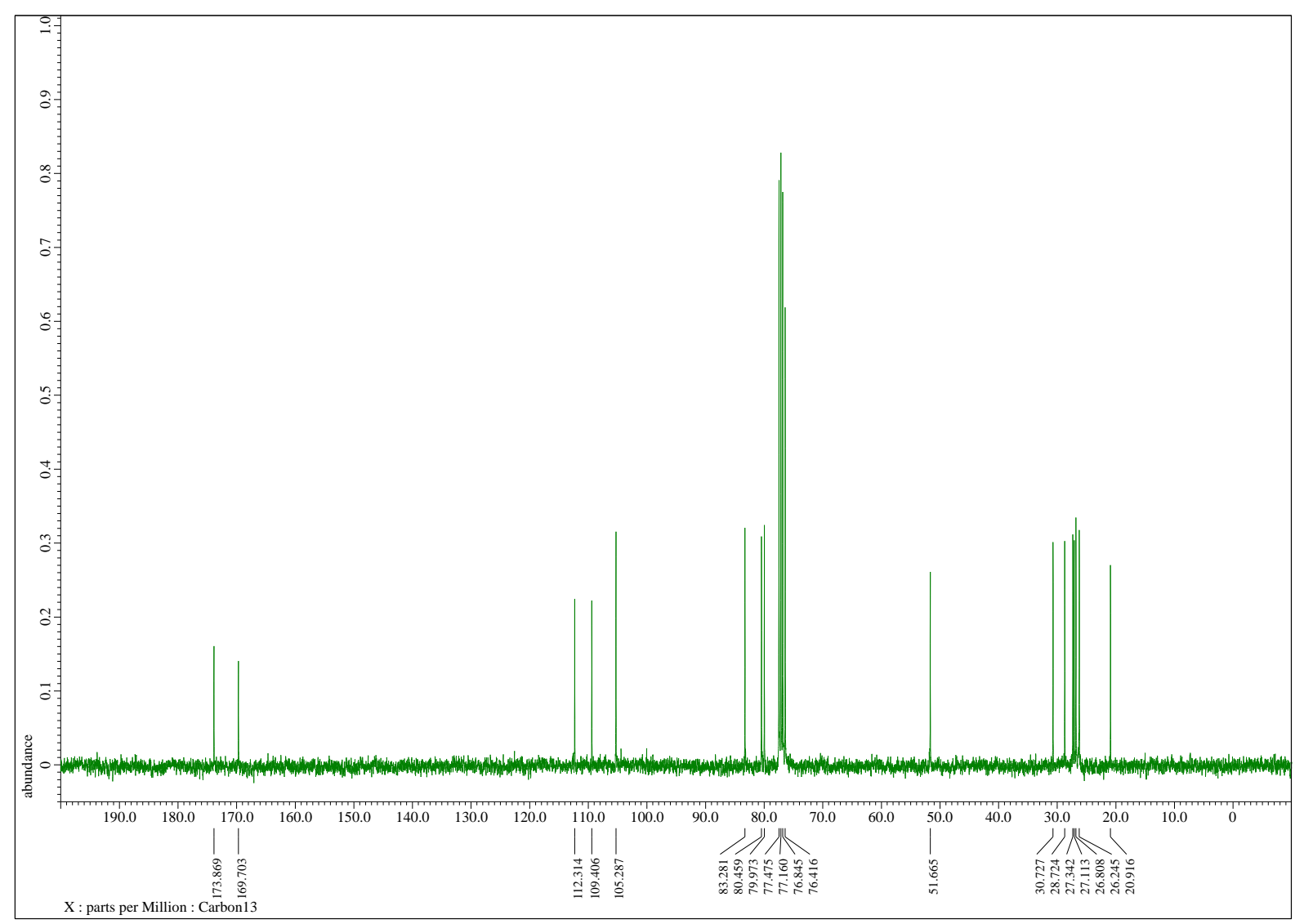


${ }^{1} \mathrm{H}$ NMR spectrum of $34\left(400 \mathrm{MHz}, \mathrm{CDCl}_{3}\right)$

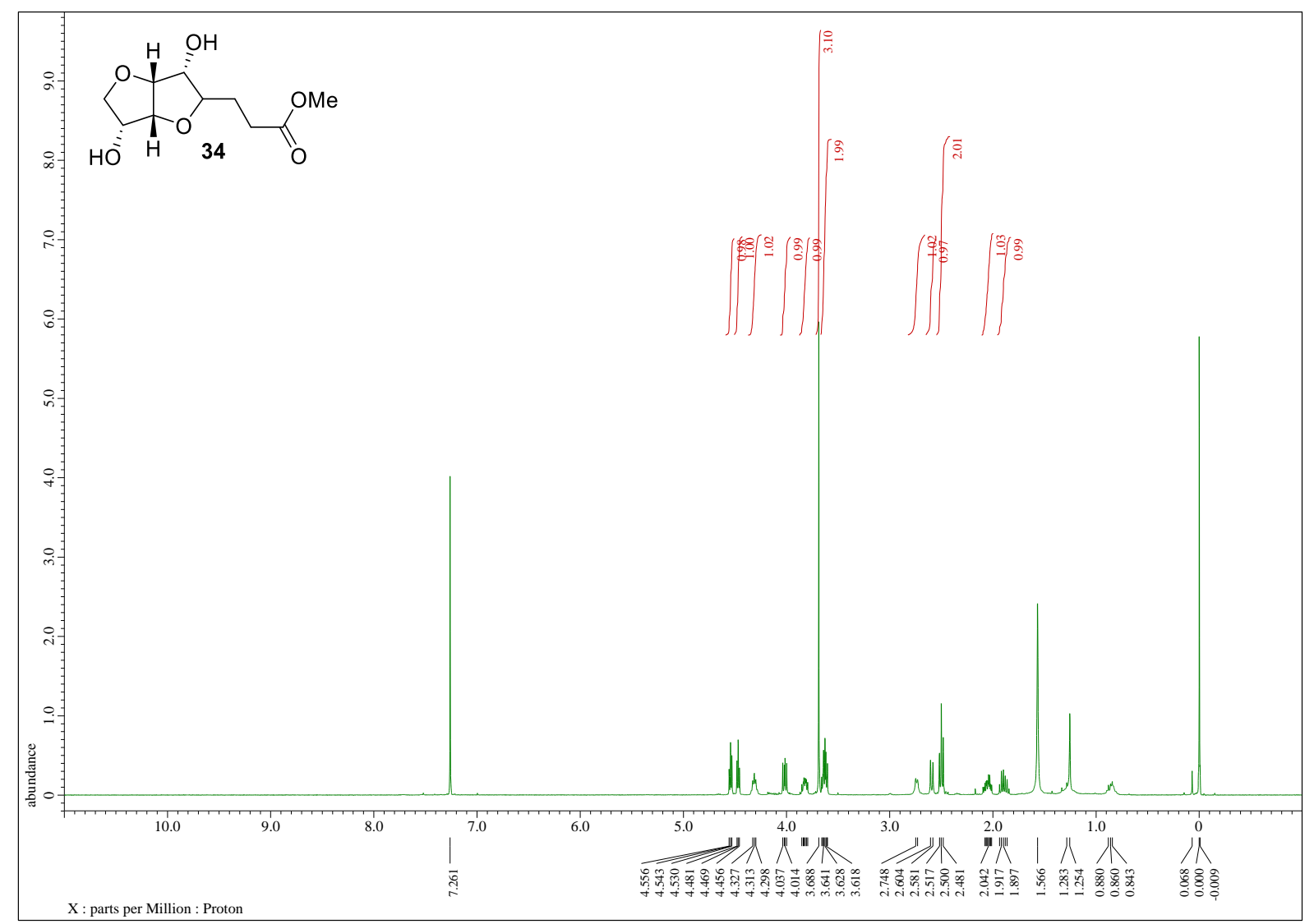

${ }^{13} \mathrm{C}$ NMR spectrum of $\mathbf{3 4}\left(100 \mathrm{MHz}, \mathrm{CDCl}_{3}\right)$

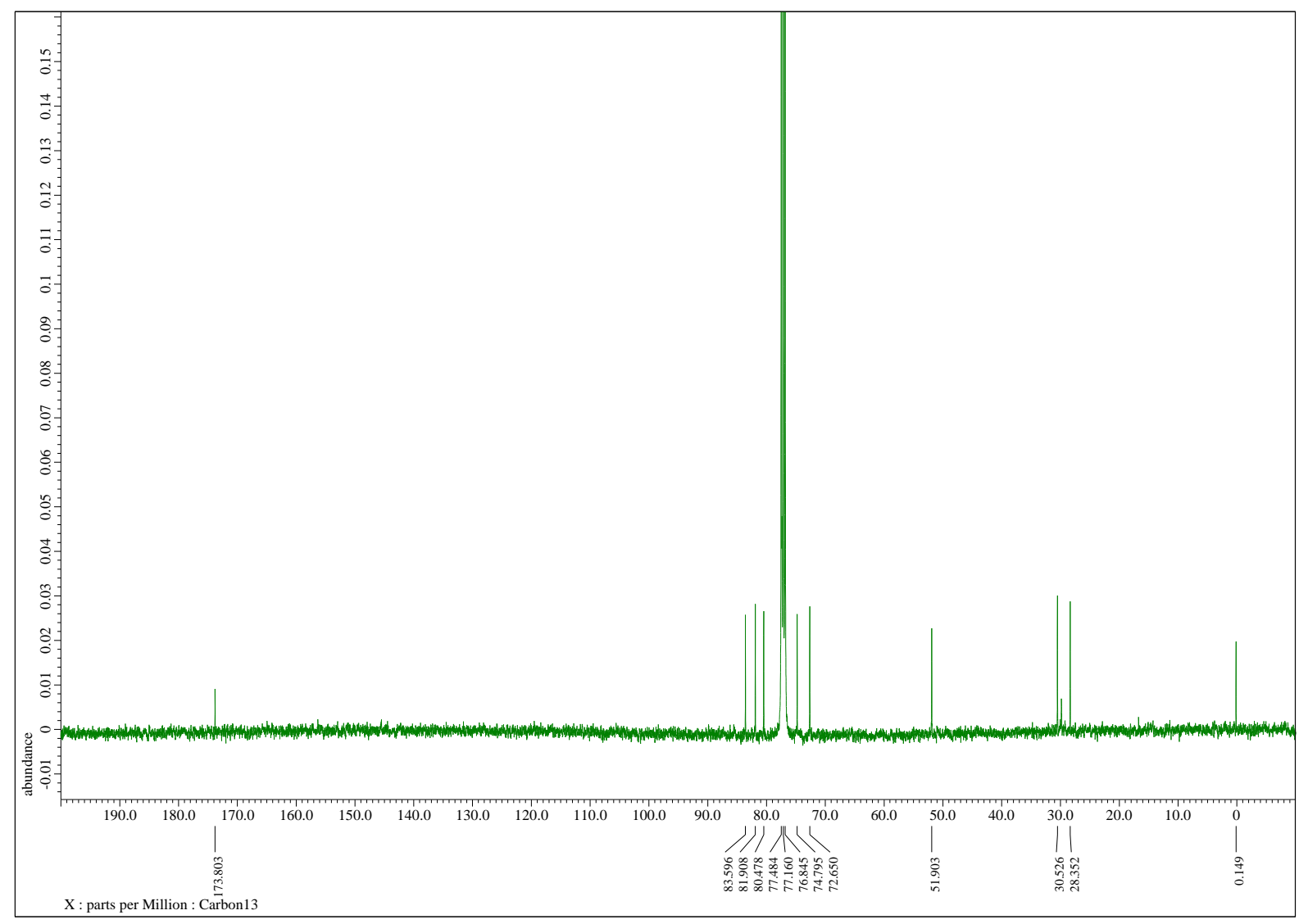

